\title{
Elastic and viscoelastic deformation of glass plates by excimer laser induced stresses and patterned silicon suboxide films
}

\author{
Dissertation \\ zur Erlangung des mathematisch-naturwissenschaftlichen \\ Doktorgrades \\ "Doctor rerum naturalium" \\ der Georg-August-Universität Göttingen \\ im Promotionsstudiengang "Physik" \\ der Georg-August University School of Science (GAUSS)
}

vorgelegt von

Clemens Martin Beckmann

aus Bremen

Göttingen, 2021 


\section{Betreuungsausschuss}

apl. Prof. Dr. Alexander Egner

(Institutsdirektor, Institut für Nanophotonik Göttingen e.V.)

apl. Prof. Dr. Michael Seibt

(IV. Physikalisches Institut, Georg-August-Universität Göttingen)

Dr. Ulrich Vetter

(Nagl \& Vetter GmbH, Göttingen)

\section{Mitglieder der Prüfungskommission}

Referent:

apl. Prof. Dr. Alexander Egner

(Institutsdirektor, Institut für Nanophotonik Göttingen e.V.)

Korreferent:

Prof. Dr. Hans-Christian Hofsäss

(II. Physikalisches Institut, Georg-August-Universität Göttingen)

Weitere Mitglieder der Prüfungskommission:

apl. Prof. Dr. Michael Seibt

(IV. Physikalisches Institut, Georg-August-Universität Göttingen)

Dr. Jürgen Ihlemann

(Abteilung Kurze Pulse/Nanostrukturen, Institut für Nanophotonik Göttingen e.V.)

Prof. Dr. Cynthia A. Volkert

(Institut für Materialphysik, Georg-August-Universität Göttingen)

Prof. Dr. Ansgar Reiners

(Institut für Astrophysik, Georg-August-Universität Göttingen)

Tag der mündlichen Prüfung: 16. September, 2021 


\title{
Elastic and viscoelastic deformation of glass plates by excimer laser induced stresses and patterned silicon suboxide films
}

\author{
Clemens Martin Beckmann
}

\begin{abstract}
A mechanically stressed film can cause a bending of the underlying substrate. Usually, the substrate deforms elastically, but in special cases a plastic or viscous deformation can be observed. Such deformations are mostly unwanted. However, methods have been developed by several people to specifically deform plate-like substrates via the generation of plane stress components inside a near-surface region, and by this to correct errors in the surface topography of thin optical components (figure correction). It was also proposed to make use of the viscous deformation of a glass substrate due to a stressed film for forming of optical components. Excimer lasers are applied in precise structuring of glass surfaces and patterning of dielectric films on glass substrates.

In this thesis, I report on the deformations and stresses in two systems related to the application of an ArF excimer laser $(193 \mathrm{~nm}, 20 \mathrm{~ns})$. The first system consists of sheets of the borosilicate glass Schott D263M. When irradiated, a large tensile stress is generated in a surface-near region. From the deformation of the samples, I measured the integrated stress in dependence on the irradiation parameters, its long-term temporal evolution and the stress distribution in direction normal to the surface. Further, I show that antibiaxial plane-stress components can be generated by irradiation with a line pattern. I performed a proof of principle for an application of the laser induced stresses in figure correction. The results also demonstrate the relevance of long-term thermal stresses in excimer laser ablation of glasses.

The second system consists of continuous or laser patterned thin films of a substoichiometric silicon oxide on plate-like substrates of vitreous silica. By high temperature $\left(>1000^{\circ} \mathrm{C}\right)$ annealing of the samples, a large viscous deformation of the substrate is induced, which depends on the film pattern. I measured the deformation for different film patterns and for different atmospheres during annealing. By comparison to the results of a finite element simulation and analytic calculations, I show that the deformation can be understood by analogy to the case of an elastic substrate and stresses due to the oxidation of the film. The results pave the way for an application for forming of plates by a patterned film.
\end{abstract}





\section{Abbreviations}

\begin{tabular}{c|l} 
abbreviation & meaning \\
\hline AFM & Atomic force microscopy \\
DIC & Differential interference contrast \\
FE(M) & Finite element (method) \\
IFNano & Institut für Nanophotonik Göttingen e. V. \\
LIPSS & Laser-induced periodic surface structure \\
PECVD & Plasma-enhanced chemical vapor deposition \\
PV & Peak-to-valley \\
RL & Reflected light \\
RMS & Root mean square \\
SCCG & Sub critical crack growth \\
SEM & Scanning electron microscope/microscopy \\
SiO & Substoichiometric silicon oxide \\
TEM & Transmission electron microscope/microscopy \\
TGM & Temperature gradient mechanism \\
UV & Ultraviolet \\
\hline
\end{tabular}





\section{Nomenclature}

\begin{tabular}{c|l} 
Symbol & Quantity \\
\hline$A$ & Area \\
$A^{\prime}$ & Parameter $A$ in $[1]$ \\
$A_{c}^{\prime}$ & Critical value of $A$ in $[1]$ \\
$A_{\mathrm{dg}}$ & Parameter $A$ of the Deal-Grove model $[2]$ \\
$a$ & Multipurpose variable \\
$a^{\prime}$ & Multipurpose variable \\
$B_{\mathrm{dg}}$ & Parabolic rate constant \\
$B_{f}$ & Biaxial modulus of the film \\
$B_{s}$ & Biaxial modulus of the substrate \\
$b$ & Line width \\
$C$ & Linear rate constant \\
$C_{p}$ & Heat capacity at constant pressure \\
$c$ & Concentration \\
$\mathcal{D}$ & Differential operator \\
$d$ & Line period \\
$E$ & Young's modulus \\
$E_{r}$ & Ratio of biaxial moduli $B_{f} / B_{s}$ \\
$F$ & Force \\
$f$ & Arbitrary function \\
$\mathbf{f}$ & Body force vector \\
$G$ & Shear modulus \\
$G_{1}$ & Shear relaxation modulus \\
$g$ & Gravitational acceleration \\
$h$ & Height \\
$I$ & Integral term \\
$k$ & Curvature \\
$k_{p}$ & Curvature along (parallel to) the lines \\
$k_{s}$ & Curvature across (orthogonal to) the lines \\
$k_{\mathrm{spring}}$ & Spring constant \\
$\mathcal{L}[f]$ & Laplace transformation of function $f$ \\
$L_{t h}$ & Thermal diffusion length \\
$l$ & Length \\
$m$ & Slope \\
$N$ & Integrated stress \\
$p$ & Unit normal vector \\
& Number or number density \\
\hline & Laplace transformation parameter \\
\hline &
\end{tabular}




\begin{tabular}{c|l}
$Q$ & Amplitude \\
$R$ & Residual \\
$r$ & Radius \\
$r_{A}$ & Area ratio \\
$r_{s}$ & Radius of cylindrical substrate \\
$s$ & Time \\
$\mathbf{T}$ & Traction \\
$T$ & Temperature \\
$T_{f}$ & Fictive temperature \\
$T_{g}$ & Glass transition temperature \\
$t$ & Time \\
$t_{f}$ & Film thickness \\
$t_{r}$ & Thickness ratio $t_{f} / t_{s}$ \\
$t_{s}$ & Substrate thickness \\
$u$ & Displacement or arbitrary field \\
$v$ & Ratio $t / \tau$ \\
$w$ & Weighting or test function \\
$x$ & Position, thickness or parameter in $\mathrm{SiO}_{x}$ \\
$y$ & Position \\
$z$ & Position \\
\hline$\alpha$ & Coefficient of linear thermal expansion \\
$\delta$ & Ratio $A / A_{c}$ in $[1]$ \\
$\eta$ & Viscosity \\
$\kappa$ & Thermal diffusivity \\
$\lambda$ & First Lamé constant \\
$\lambda_{c}$ & Thermal conductivity \\
$\mu$ & Second Lamé constant \\
$\nu$ & Poisson's ratio \\
$\rho$ & Aspect ratio \\
$\rho_{m}$ & Mass density \\
$\sigma$ & Stress \\
$\tau$ & Relaxation time \\
$\tau_{L}$ & Laser pulse duration \\
$\tau_{p}$ & Structural relaxation time \\
$\phi$ & Basis function \\
\hline & Function $\chi$ in $[3$ \\
\hline
\end{tabular}




\section{Contents}

\begin{tabular}{lll}
\hline 1 & Introduction & 13
\end{tabular}

2 Materials and methods $\quad 23$

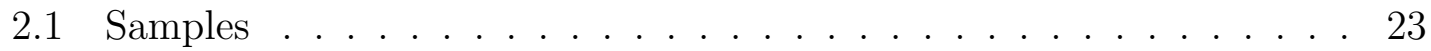

2.2 Thin film deposition $\ldots \ldots \ldots \ldots \ldots \ldots$

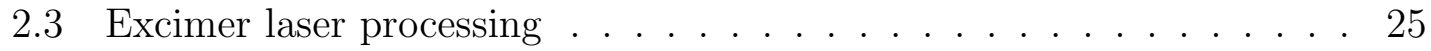

2.4 Tactile profilometry $\ldots \ldots \ldots \ldots \ldots \ldots \ldots \ldots \ldots$

2.5 Transmission and reflection measurements . . . . . . . . . . . 32

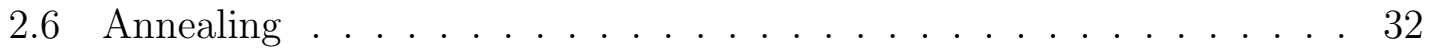

2.7 Etching experiments $\ldots \ldots \ldots \ldots \ldots \ldots \ldots$

2.8 Finite element simulations $\ldots \ldots \ldots \ldots \ldots \ldots$

2.8.1 Introduction to the finite element method and the FEniCS

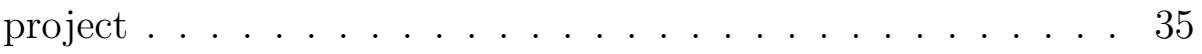

2.8 .2 The linear elastic simulation . . . . . . . . . . . . . . . 37

2.8 .3 The linear viscoelastic simulation . . . . . . . . . . . . 38

3 Elastic deformation of borosilicate glass plates by laser induced stresses 41

3.1 Experimental results $\ldots \ldots \ldots \ldots \ldots \ldots$

3.1.1 Example for deformation due to irradiation. . . . . . . . . . 41

$3.1 .2 \quad$ Integrated stress in dependence on fluence, pulse number and

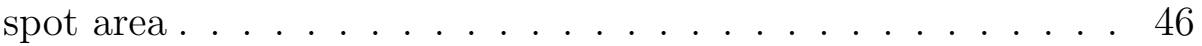

$3.1 .3 \quad$ Etching in potassium hydroxide solution $\ldots \ldots \ldots \ldots$ 
3.1.4 Antibiaxial plane stress component by line pattern . . . . . . 51

3.1.5 Long-term temporal evolution of the integrated stress . . . . . 55

3.1 .6 Application for figure correction . . . . . . . . . . . . . . 56

3.2 Discussion $\ldots \ldots \ldots \ldots$. . . . . . . . . . . . . . . . . . . 61

3.2 .1 The origin of the stress . . . . . . . . . . . . . . 61

3.2 .2 Stability of the integrated stress and crack formation . . . . 65

3.2 .3 Generation of antibiaxial stress components . . . . . . . . . 66

$3.2 .4 \quad$ A method for figure correction? . . . . . . . . . . . . . 68

4 Theoretical considerations for the linear viscoelastic deformation $\begin{array}{ll}\text { of plates } & 73\end{array}$

4.1 Deformation by a stressed film . . . . . . . . . . . . . . . 73

4.1 .1 The viscoelastic Stoney equation . . . . . . . . . . . 75

4.1.2 The viscoelastic version of the equation of Brenner and Senderoff 80

4.1.3 The viscoelastic version of the equation of Wikström et al. . . 83

4.2 Deformation by gravity . . . . . . . . . . . . . . . . . . . . . . 85

$4.2 .1 \quad$ Bearing at the lower edge . . . . . . . . . . . . . 87

$4.2 .2 \quad$ Bearing at two opposite points . . . . . . . . . . . . . 87

5 Viscoelastic deformation of fused silica plates covered with a con$\begin{array}{lc}\text { tinuous or patterned silicon suboxide film } & 89\end{array}$

5.1 Results on the viscous deformation . . . . . . . . . . . . . . . . . 89

5.1.1 Annealing in air at $1120^{\circ} \mathrm{C}$ in horizontal orientation . . . . . . 89

$5.1 .2 \quad$ Annealing in air at $1120^{\circ} \mathrm{C}$ in vertical orientation . . . . . . . 96

$5.1 .3 \quad$ Annealing in different atmospheres . . . . . . . . . . . . . . . 99

$5.1 .4 \quad$ Annealing of line patterns . . . . . . . . . . . . . . 102

5.2 Additional results . . . . . . . . . . . . . . . . . . . . . 112

5.2.1 Stress change of silicon suboxide films due to annealing . . . . 112

5.2.2 Stresses induced by laser ablation of silicon suboxide films . . 114 
5.2 .3 Oxidation kinetics $\ldots \ldots \ldots$. . . . . . . . . . . . . . . . .

5.2.4 Deformation of vitreous silica cover glasses during oxidation at $900^{\circ} \mathrm{C} \ldots \ldots \ldots \ldots \ldots$

5.3 Discussion . . . . . . . . . . . . . . . . . . . . . . 121

5.3 .1 The origin of the film stress . . . . . . . . . . . . . . . 121

5.3 .2 Deformation and gravity . . . . . . . . . . . . . . . . 127

5.3 .3 Line structured films . . . . . . . . . . . . . . . . . . . . . . . 129

$5.3 .4 \quad$ Application of the viscous deformation . . . . . . . . . . . . 131

5.3 .5 Outlook . . . . . . . . . . . . . . . . . . 134

\begin{tabular}{lll}
\hline 6 & Conclusion & 137
\end{tabular}

\begin{tabular}{ll}
\hline A Material properties and constants & 139
\end{tabular}

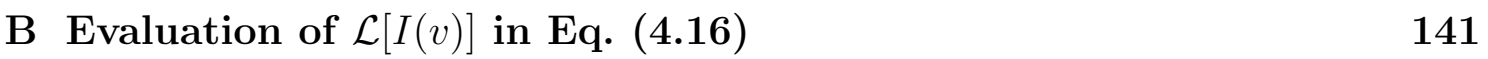

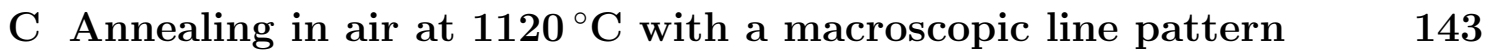

C.1 Horizontal sample orientation . . . . . . . . . . . . . . . . . . . 143

C.2 Vertical orientation . . . . . . . . . . . . . . . 146

\begin{tabular}{lr}
\hline Bibliography & 149
\end{tabular}

\begin{tabular}{ll}
\hline Acknowledgments & 165
\end{tabular} 



\section{Chapter 1}

\section{Introduction}

It is a common issue of thin film technology that the films are stressed after deposition [4, 5]. Such film stresses can lead to film cracking [6], delamination of the film [7, 8] or even to a significant bending of the underlying substrate [5, 9, 10]. In case of bending of the substrate, a compressive/tensile film stress leads to a convex/concave deformation of the coated side of the substrate (Fig. 1.1] [4]. For a continuous and homogeneous film of stress $\sigma_{f}$ and thickness $t_{f}$ on a plate-like substrate of thickness $t_{s}$, the film-substrate system develops a homogeneous curvature if the deflection of the substrate is much smaller than its thickness [1]. If further $t_{f} \ll t_{s}$ and the substrate is mechanically isotropic and homogeneous, the curvature can be calculated by the Stoney equation 11

$$
k=\frac{6\left(1-\nu_{s}\right)}{E_{s}} \frac{t_{f}}{t_{s}^{2}} \sigma_{f}
$$

where $E_{s}$ and $\nu_{s}$ are the Young's modulus and the Poisson's ratio of the substrate, respectively. The curvature $k$ is proportional to the film stress $\sigma_{f}$ and the film thickness $t_{f}$ and is inversely proportional to the square of the substrate thickness $t_{s}$. The integral $N=\int_{0}^{t_{f}} \sigma_{f}(z) \mathrm{d} z$ ( $z$ direction: normal to surface) is the integrated film stress (or line stress) and is used to quantify the overall force that the film exerts on the substrate.

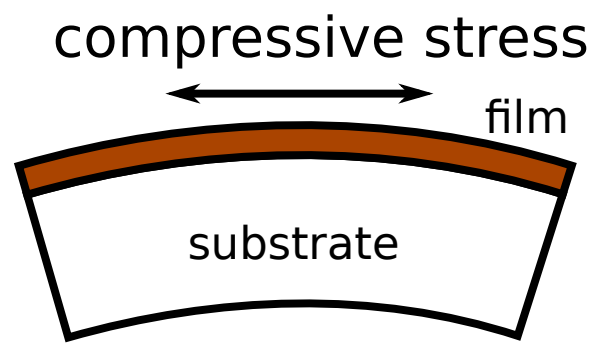

\section{tensile stress}

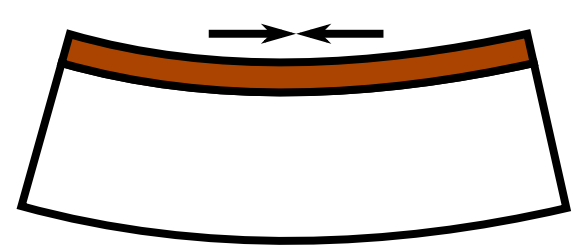

Figure 1.1: A compressive film stress means that the film is hindered in its lateral expansion by the substrate. Therefore, a convex sample curvature evolves. In contrast, a film with a tensile stress tends to reduce its lateral dimensions and causes a concave curvature.

A more thorough analysis of the deflection of a substrate by a stressed film 
was performed by Brenner and Senderoff [12]. They distinguished between different boundary conditions for the substrate during thin film deposition, considered the influence of the film thickness $t_{f}$ and of different elastic properties $E$ and $\nu$ of film and substrate. From Eq. (33) of [12], an approximate equation

$$
k=\frac{6\left(1-\nu_{s}\right)}{E_{s}} \frac{t_{f}}{t_{s}^{2}} \sigma_{f} \frac{1}{1+4 E_{r} t_{r}-t_{r}}
$$

was derived in [13 for the curvature in the case of a finite film thickness $t_{f}$. Here, $t_{r}=t_{f} / t_{s}$ is the thickness ratio of film and substrate, and $E_{r}=B_{f} / B_{s}$ is the ratio of the biaxial moduli $B_{i}=E_{i} /\left(1-\nu_{i}\right)$ for $i=f, s$. Although the stress-curvature relation is still linear, the slope is changed by a correction factor that considers the thickness ratio $t_{r}$ and the ratio of biaxial moduli $E_{r}$. This correction factor is plotted in Fig. 1.2 for realistic values of $t_{r}$ and $E_{r}$.

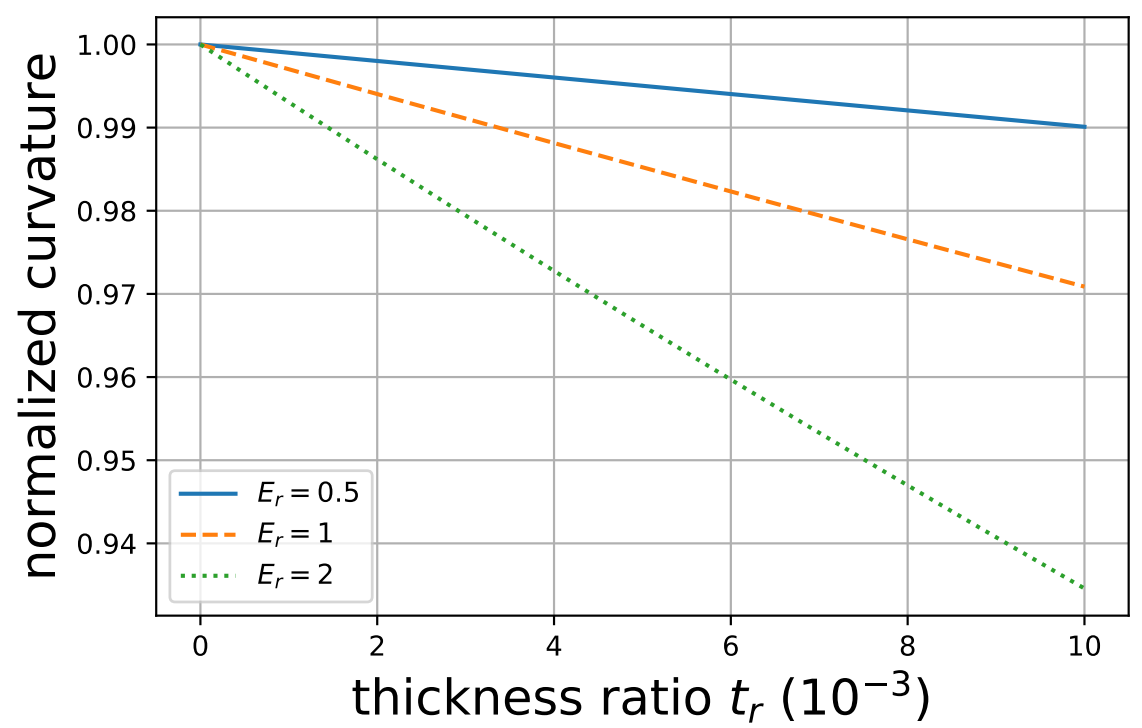

Figure 1.2: The correction term of the Brenner-Senderoff Eq. 1.2 , i. e. the predicted curvature normalized by the curvature calculated by the Stoney Eq. (1.1), in dependence on the thickness ratio $t_{r}$ for different ratios of biaxial moduli $E_{r}$.

In many technologically important cases, not only a single film but a film stack (multilayer) is deposited. For that case, it was demonstrated that the overall curvature is the superposition of the curvatures each individual layer would induce [14].

More complex behavior of the curvature was observed for the case of large deflections [1, 13, 15, 16]. In [1], the ratio $\delta=A^{\prime} / A_{c}^{\prime}$ is introduced with $A^{\prime}=4 \cdot N \cdot r_{s}^{2} / t_{s}^{3}$, $A_{c}^{\prime}$ denoting a critical value of $A$ and $r_{s}$ being the lateral radius of a round, platelike substrate. Three regimes for $\delta$ are distinguished: For small values of $\delta \lesssim 0.2$, the Stoney Eq. (1.1) is valid and the curvature is uniform. For intermediate values $0.2 \lesssim \delta<1$, the curvature would be overestimated by the Stoney equation and becomes non-uniform, i. e. its magnitude is smallest at the center and largest at the rim of the sample. At $\delta=1$, a bifurcation of the curvature is observed. In one direction it increases with increasing $\delta$ while in the orthogonal direction it decreases. 
The above considerations are based on the assumption of a film of laterally uniform thickness and laterally uniform equibiaxial stress. However, in many applications, the film thickness or the film stress are non-uniform. The case of a non-uniform film thickness and a laterally non-uniform isotropic strain inside the film is studied theoretically in [17]. It is demonstrated that the relations between the film stress and the system curvature are non-local, i. e. that a local film stress can affect the curvature at other positions of the substrate surface. The calculated relations were verified in 18$]$.

A special case of a non-uniform film thickness distribution is a continuous film patterned into periodic lines of height $t_{f}$, width $b$ and distance $d$ (Fig. 1.3a). This case was studied in $3,19,20$ for stresses caused by the difference in thermal expansion of substrate and film (thermal stresses). It was found that patterning of the film leads to a laterally anisotropic curvature of the system, that is described by a large absolute curvature value $\left|k_{p}\right|$ in direction along the lines and a smaller absolute curvature value $\left|k_{s}\right|$ in direction across the lines [19] (Fig. 1.3b). The ratio $k_{s} / k_{p}$ decreases with increasing aspect ratio $\rho=t_{f} / b$ of the lines and even becomes negative for large $\rho$. For $\rho \rightarrow \infty$, it approaches $k_{s} / k_{p}=-\nu_{s}$, while the stress $\sigma_{s}$ in the lines in direction across them approaches zero [3, 19]. In [3], an analytic theory was derived, that can be considered as an extension of the Stoney Eq. (1.1). According to this theory, the curvatures

$$
\mathbf{k}=\left(\begin{array}{c}
k_{p} \\
k_{s} \\
2 k_{p s}
\end{array}\right)
$$

are given by

$$
\mathbf{k}=\frac{6\left(1-\nu_{s}^{2}\right)}{E_{s}} \frac{t_{f}}{t_{s}^{2}} \sigma_{f} \frac{b}{d} \mathbf{P}_{s}^{-1}\left(\begin{array}{c}
1-\chi(\rho) \nu_{f} \\
1-\chi(\rho) \\
0
\end{array}\right)
$$

where $\sigma_{f}$ is the stress inside the film before patterning, $\chi(\rho)$, which was derived by analogy to a cracked composite material [21], is a function of the aspect ratio $\rho$ of the line structures and

$$
\mathbf{P}_{s}=\left(\begin{array}{ccc}
1 & \nu_{s} & 0 \\
\nu_{s} & 1 & 0 \\
0 & 0 & \left(1-\nu_{s}\right) / 2
\end{array}\right)
$$

Because the deformation of a substrate due to the deposition of a functional film can lead to a degradation of the part's quality [5, 9, 10], different approaches exist to reduce the amount of deformation. The intuitive approach is the reduction of the film stress by optimization of the deposition process [5] or by post-deposition treatment, like annealing of the film-substrate system 22 24]. Another common approach is the choice of thick substrates because the curvature $k$ decreases by $1 / t_{s}^{2}$ (cf. Stoney Eq. (1.1) ) [5. The side effects are increases in weight, volume and material costs. There are, however, applications where at least one of these parameters needs to be small. A current example is the Lynx x-ray observatory, a mission concept under 


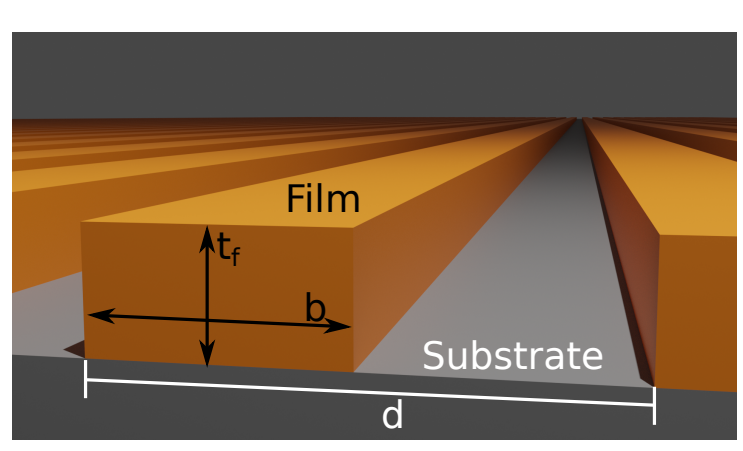

(a)

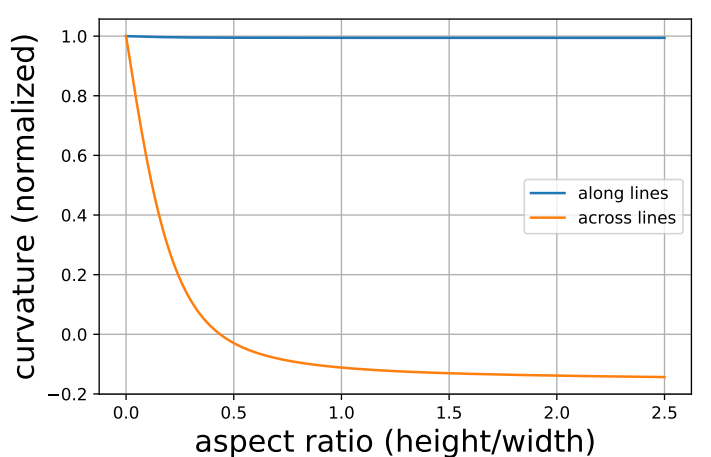

(b)

Figure 1.3: (a) Scheme of the line geometry in the theory of Wikström et al [3]. A continuous film of thickness $t_{f}$ has been patterned into lines of width $b$ and period d. (b) The curvatures, normalized by the curvature for a continuous film with consideration of the duty cycle $b / d$, for a system of a line patterned film of $\mathrm{SiO}_{x}$ on $\mathrm{SiO}_{2}$ calculated by the model. The material parameters in Tab. A.1 have been used.

consideration by the National Aeronautics and Space Administration (NASA) 25]. Its Wolter-Schwarzschild optics would consist of 37492 thin mirror segments, that need to be lightweight for launch into space and thin because of optical issues.

The third common approach to reduce the deformation is the deposition of a compensating coating [5, 9, 24]. This is a film of specific integrated stress that ideally causes the inverse deformation of the substrate, i. e. compensates the mechanical influence of the functional film (Fig. 1.4). It can be deposited opposite to the functional film on the backside of the substrate. In that case, a film with the same integrated stress as the functional film is needed, which is why often the deposition of the functional film is repeated for the backside. If the application allows it, the compensating coating can also be deposited on the same side as the functional film. In that case, an integrated stress of opposite sign is needed. The application of a compensating coating requires a simple geometry of substrate and film, if the coating is deposited on the opposite side to the functional film, and a good reproducibility of the integrated stress of the functional coating and the compensating coating. The condition of a simple geometry is violated, if both sample sides are not identical. An example for such an optical component, for which the approach of a backside coating would fail, is given in [5] by a laser mirror with a through-hole of $45^{\circ}$ inclination to the flat front side. Besides film stress, other reasons for a large surface (figure) error can be stresses introduced or removed during substrate manufacturing [26, 27], stresses induced by the holder of the optical part $[28]^{1}$ and even the gravitational influence 9,28 .

To correct for more complex figure errors, different approaches have been pursued. A powerful method is ion beam figuring (IBF), that is based on local removal

\footnotetext{
${ }^{1}$ Recently, I found that Edmund Optics, an established distributor of optical components, sells so called stemmed mirrors to reduce the deformation of the mirror surface when mounted [29]. This shows that deformation by the mount is not just an academic problem.
} 


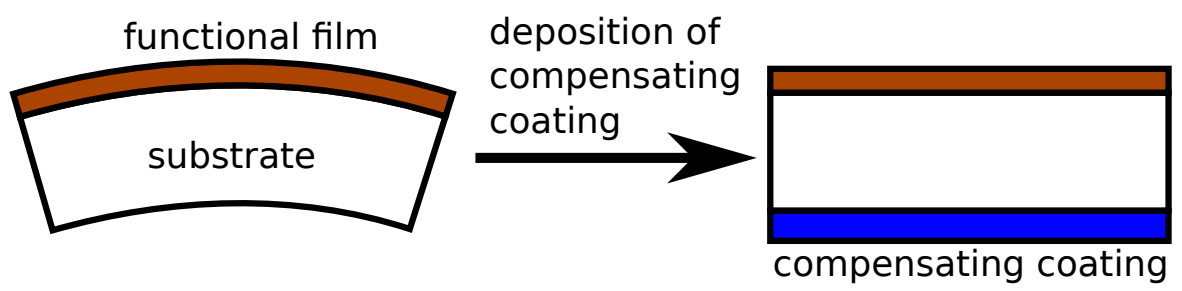

Figure 1.4: The deformation of a sample due to the deposition of a functional film can, in certain instances, be compensated by deposition of a film with the same integrated stress on the opposite side.

of material via ion beam sputtering [30]. Instead of removal, material can also locally be added to the surface [31, 32]. This is achieved by local variation of the deposition rate of a coating. The idea of a compensating coating on the backside of the substrate has also been seized, refined and generalized to different kinds of surfacenear, laterally varied stresses [33 37]. Recently, the possibilities of such methods for deformation of a thin and flat plate have been analytically studied [38]. For this, the plane stress state at every point of the surface is decomposed into an equibiaxial, an antibiaxial and a shear component (Fig. 1.5). It was found that by applying only equibiaxial stress states, the space of accessible deformations is limited. However, by adding non-equibiaxial stress states, arbitrary deformations can be reached, within the framework of the assumptions of [38]. Please note that there always exists a coordinate system in which the shear stresses are zero [39], [40, Sec. 4.3.2]. Thus, a method for figure correction is fully equipped if equibiaxial and antibiaxial plane stress states of both signs and arbitrary magnitude can be generated.

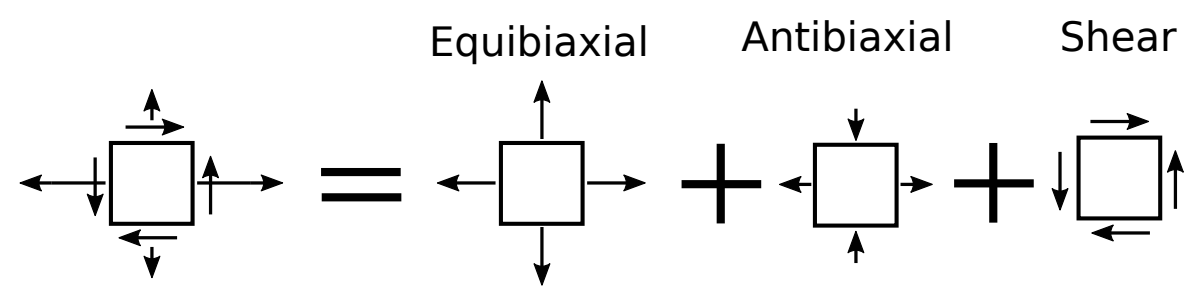

Figure 1.5: A general plane stress state can be decomposed into an equibiaxial, an antibiaxial and a shear stress component. Figure according to 38].

Near-surface stresses can be generated by ion implantation, which was studied as a figure correction method for silicon and glass substrates [36, 39]. For correction of silicon substrates, $\mathrm{Si}^{++}$ions of $2 \mathrm{MeV}$ energy were implanted inside the back surface [36]. Implantation under normal incidence leads to an equibiaxial compressive stress, that subsequently needs to be stabilized by annealing at $120^{\circ} \mathrm{C}$ for $4 \mathrm{~h}$. For the borosilicate glass Schott D263, the generation of antibiaxial plane stress states for inclined incidence was also reported [39]. Drawbacks of the method are the complex instrumental setup (tandem accelerator) and the irradiation in vacuum.

Another method, especially developed for silicon wafers, is based on the oxidation of the backside surface of the wafer [37, 41]. This leads to a compressive film stress. By lithographically patterning the oxide layer, a specific deformation can be reached. Although, to the best of my knowledge, only the generation of equibiaxial 
stress components has been demonstrated, also antibiaxial components should be achievable by a fine patterning of the layer [19]. However, the process requires the fabrication of a unique plastic film photomask for every sample and involves the application of hydrofluoric acid.

By application of a laser for figure correction, the corresponding process could be easily adapted to the current sample by calculation of the irradiation pattern, ideally no application of chemicals would be needed and ideally irradiation would be possible in ambient air. Such a process has been demonstrated in [35] for figure correction of fused silica substrates. By focusing a picosecond laser with a wavelength of $524 \mathrm{~nm}$ inside the material, non-linear absorption leads to the generation of stress centers. In-plane anisotropic stresses [42] and stresses with both signs [43] can be produced. However, because the stress centers are written one by one, it presumably is a slow process for processing large areas.

Another kind of laser based process could be the structuring of a stressed coating via laser ablation [44, 45]. Such a process was demonstrated in [46] for deformation of silicon substrates by ablation of a grown oxide layer via irradiation with a fs laser at $2.4 \mu \mathrm{m}$ wavelength.

A common method for generation of stresses by laser irradiation is the temperature gradient mechanism (TGM, [47, Sec. 3.2.1] and [48], Fig. 1.6). The laser light is absorbed in a surface-near region of the sample and converted into thermal energy. The material melts, expands and deforms plastically. During cool down, after the laser spot has moved on or the pulse is over, the molten material re-solidifies and contracts. Therefore, a tensile stress is generated in the irradiated area. Usually the TGM is used for bending of plates along a line [49 53, but it has also been used for 3D forming by following a more complex path with the laser beam [54]. Although usually metallic materials are bent, it has also successfully been applied to glasses [50, 51].

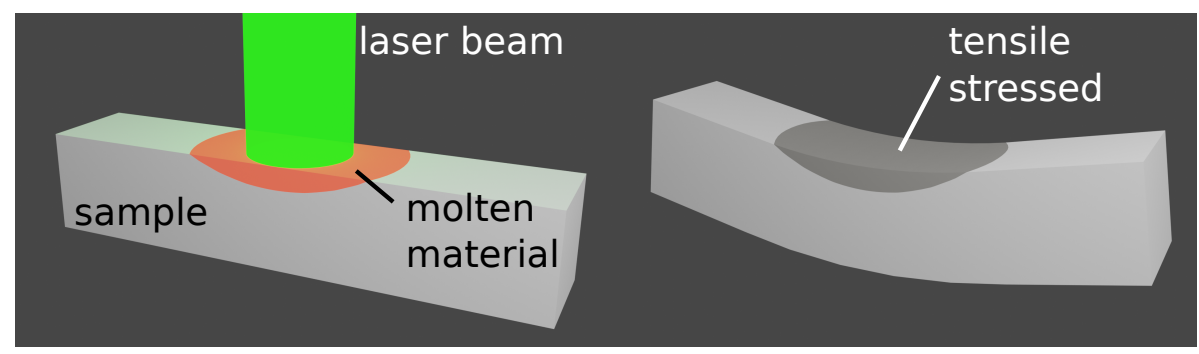

Figure 1.6: In the temperature gradient mechanism a sample is irradiated by a continuous wave or pulsed laser, that causes a local melting of the sample material (left). After the irradiation, the molten material cools down, solidifies and develops a tensile stress because of thermal contraction (right). The stress causes a deformation of the sample.

Excimer lasers are pulsed gas lasers, that usually emit ultraviolet light with pulse durations in the nanosecond regime [55, Sec. 2.1.6]. The small wavelength of the emitted light causes excellent absorption in many materials and allows for a high optical resolution. Additionally, they exhibit large pulse energies of up to $1 \mathrm{~J}$ [56] 
and a nearly flat-top fluence profile of the output beam. Therefore, large areas can be processed in parallel [57]. For the structuring of surfaces via laser ablation, these properties allow for ablation of a precisely defined area with a well defined lateral fluence profile of the laser spot [58]. Thus, smooth features can be realized. This is an advantage over ultrashort pulse lasers, which are usually focused to a small spot of Gaussian fluence distribution, that is scanned over an area for material ablation. By focusing and scanning, stitching artifacts will evolve (see e. g. the patterns in [59]). Unfortunately, the relatively long pulse durations of excimer lasers, if not a femtosecond excimer is used, cause a significant thermal load of the surrounding area by thermal diffusion, that can lead to a pronounced degradation of structure quality for the case of materials with a high thermal conductivity, like metals 60 , Ch. 3]. Therefore, excimer lasers are especially suited for the structuring of dielectric materials, like silicate glasses, that are transparent in the visible spectral range but exhibit a sufficient absorption in the UV range and a low thermal diffusion 57,58 , 61 67]. In [68], it was found that laser ablation of fused silica with the light of an $\mathrm{F}_{2}$ excimer laser (157 nm wavelength, $15 \mathrm{~ns}$ pulse duration) leads to tensile stresses inside the ablated surface. The authors assume that the stresses are created by a similar mechanism as the TGM. Surprisingly, to my knowledge, this is the only study that takes a closer look at the stress generation in glasses during structuring with excimer lasers. Because laser generated stresses are related to crack formation and the strength of the material [69 71], a better understanding of the stresses induced during laser ablation could lead to an improved quality of structures produced by ablation. Additionally, laser induced stresses can be used for selective deformation 45].

In section 3.1 of the present work, I show experimental results on the generation of tensile stresses inside the borosilicate glass Schott D263M by irradiation with the light of an ArF excimer laser (193 nm wavelength, $\approx 20 \mathrm{~ns}$ pulse duration). I measured the integrated stress in dependence on the laser fluence and the stress distribution inside the stressed surface layer in direction normal to the surface. Large stresses of up to $400 \mathrm{MPa}$ are generated by the TGM. These results confirm the observations and interpretations made in $[68]$ for the stresses generated in fused silica during ablation with an $\mathrm{F}_{2}$ excimer laser. I demonstrate that antibiaxial plane stress components can be generated by structuring the surface into a line pattern. Line patterns on glass can be used for diffraction of light [67], light-coupling [72] or for functionalization of surfaces [73]. My finding could lead to a better understanding of the mechanical strength (the maximum stress a material can withstand) of line patterned surfaces and therefore could be relevant for the mentioned applications. I also show that the magnitude of the generated stress decreases with time for storage in air. Nevertheless, I propose that the TGM could be used for figure correction of glass substrates. By use of an excimer laser, large areas of homogeneous, equibiaxial plane stress could be generated inside a surface layer of a glass sample by irradiation with one pulse per position. For generation of antibiaxial plane stress components, a large spot with a line structured fluence distribution could be used.

Up to this point, I only considered the elastic deformation of the underlying substrate by a stressed surface layer. However, in certain cases, a viscous or plastic deformation of substrates was observed. In [74], the curvature of silicon wafers, covered on one side with a silicon nitride film, was measured for annealing at different 
temperatures. It was found that the curvature increased by creep of the wafer. A plastic deformation of silicon wafers at elevated temperature was also observed in [75] for diamond films and in [76] for films of TiN and TiAlN. In [77], the creep of a steel foil during oxidation was observed, and in [78], the curvature increase by plastic deformation of germanium wafers covered on one side by a silicon film is reported.

In [79], the curvature evolution of a film-substrate system was simulated for the case of a viscoelastic deformation of the substrate described by the Maxwell model (cf. Sec. 2.8.3) of a viscoelastic fluid. It was found that the ratio $t_{f} / t_{s}$ for given film and substrate materials decides whether the magnitude of the curvature of the film-substrate system increases or decreases during annealing. For $t_{f} \ll t_{s}$, the case of a thin film on a thick substrate, it increases. Analytic equations for the curvature evolution in case of viscous flow of the substrate are given in [80] and [81] for quite general cases.

To my knowledge, the viscous or plastic deformation of substrates due to a structured film has not systematically been studied, yet. Plus, there is a lack on experimental studies on the viscoelastic deformation of glass substrates due to a stressed film. Both aspects are considered in the present work in Chs. 4 and 5 . They are important from a technological point of view because in many applications noncontinuous films are applied, sometimes films need to be annealed or deposited at high temperatures and silicate glasses are an important class of substrate materials. For example, the production of a piezoelectric layer on a glass substrate involves a crystallization step at high temperature, that can cause a permanent deformation of the substrate 82 .

Excimer lasers are not only well suited for the precise structuring of glass surfaces, as was mentioned above, but also for the structuring of dielectric films 83 85. An especially good structure quality can be obtained by the so called backside (or rear-side) ablation configuration if the substrate is sufficiently transparent (Fig. 1.7) 83, 86, 87]. In backside ablation, the laser beam is transmitted by the substrate and the light is absorbed inside the film material, close to the film-substrate interface. At sufficiently high fluences, the adhesion of the film to the substrate and the strength of the film are overcome, and the film is removed inside the irradiated area. If the fluence is not too high, the damage of the uncovered substrate surface is small [87, 88]. A common system, that is structured this way in our group, is a film of substoichiometric silicon oxide (silicon suboxide or $\mathbf{S i O}_{x}$ ) on a fused silica substrate [87]. At the common excimer wavelengths of 193, 248 and $308 \mathrm{~nm}$, the fused silica substrate is highly transparent, but the $\mathrm{SiO}_{x}$ strongly absorbing [84. This allows for the fabrication of $\mathrm{SiO}_{x}$ microstructures with steep edges and small surface roughness [85]. By a subsequent annealing step of the sample at temperatures of $900^{\circ} \mathrm{C}$ and above in oxidizing atmosphere, the $\mathrm{SiO}_{x}$ can be transformed to $\mathrm{SiO}_{2}$. Thus, afterwards a monolithic fused silica sample is obtained, that e. g. can be used as a phase mask in optical applications [89].

For such samples of a laser structured $\mathrm{SiO}_{x}$ film on a fused silica substrate, large absolute values of curvature were observed after oxidation of the film [90]. Further, it was observed that, in case of a line structured film, the magnitude of the curva- 


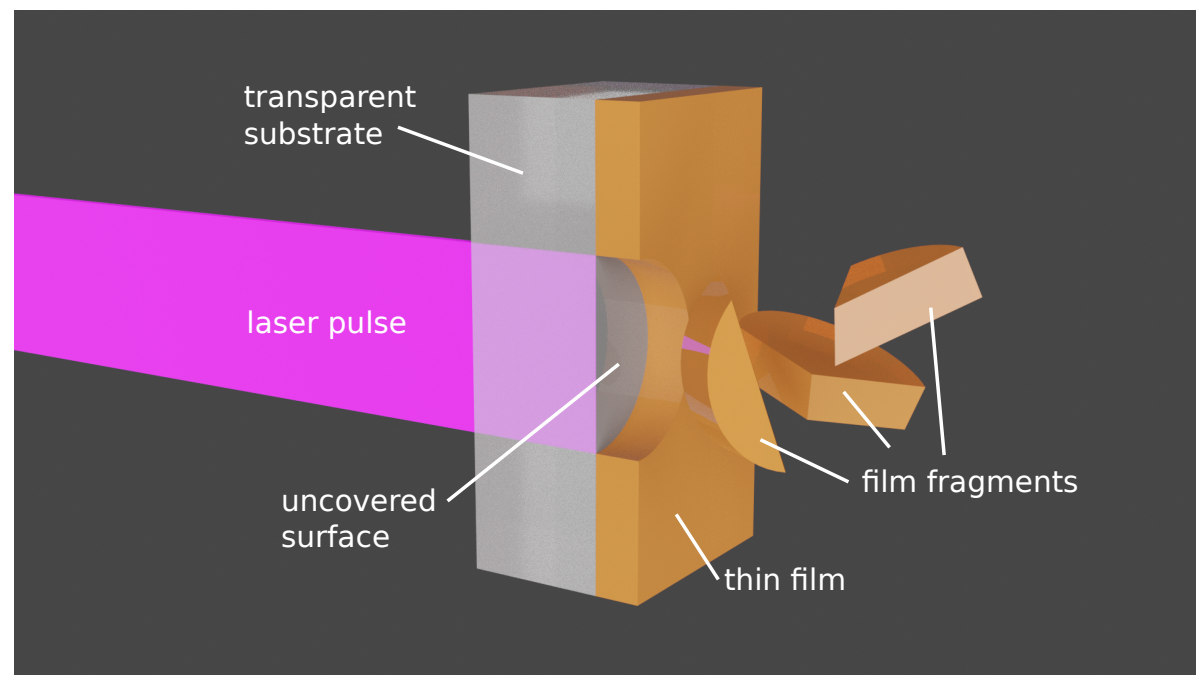

Figure 1.7: In backside ablation of thin films, the laser light is transmitted by the transparent substrate and absorbed at the substrate/film interface.

ture is larger in direction along the lines than across the lines. Therefore, it was assumed that complex viscoelastic deformations of the substrate can be achieved by a structured film, and a method based on structuring and oxidation of substoichiometric oxide films was proposed for the production of optical components [90]. Such a component could be a freeform, aspheric or off-axis mirror with small absolute curvature values, where a freeform surface is defined as a surface with no axis of rotational invariance [91]. Because the use of freeform optics can lead to a reduction in complexity, volume and weight of optical setups, they are relevant for many different fields of application [91].

In Sec. 5.1 of this thesis, I report on the experimental results for the viscoelastic deformation of fused silica plates by a continuous or laser patterned $\mathrm{SiO}_{x}$ film. Before that, in Sec. 4.1. I derive simple equations for a better understanding and interpretation of the experimental results. I annealed samples at $1120^{\circ} \mathrm{C}$ in air and at $1060^{\circ} \mathrm{C}$ in oxygen and almost inert atmosphere. Qualitatively, the deformation is similiar as would be expected in the elastic case, as is verified by finite element (FE) simulations, but the attained absolute values of curvature are much larger. A compressive integrated film stress of roughly -10 to $-20 \mathrm{~N} / \mathrm{m}$ is observed, that presumably is generated by oxidation. Gravity seems to have a large influence on the deformation, in that it causes a decrease in the absolute curvature after complete oxidation and a non-uniform curvature. I also studied the behavior of $\mathrm{SiO}_{x}$ films at lower temperatures, of which the results are presented in Sec. 5.2. I found that backside ablation introduces a tensile stress inside the uncovered fused silica surface, which could be caused by the TGM. I also found that oxidation in oxygen at $920^{\circ} \mathrm{C}$ is diffusion limited and proceeds faster than for a silicon surface. This work is a first step in direction to a method for forming of plates by patterned films, as it was proposed in 90$]$. 



\section{Chapter 2}

\section{Materials and methods}

As part of this work, I applied an ArF excimer laser for irradiation of samples of Schott D263M and for patterning of thin films of $\mathrm{SiO}_{x}$ deposited on silica substrates. I measured the deformation of the samples by tactile profilometry and annealed some of the samples inside a muffle furnace or inside a tube furnace in different atmospheres. For a better understanding of the experimental results, I performed a linear elastic and a linear viscoelastic FE simulation by use of the FEniCS project. In this chapter, I describe my samples and the applied methods in detail.

\subsection{Samples}

The samples used in this study can be classified in cover glasses of Schott D263M, round plates of Heraeus Suprasil, and cover glasses of GE124.

I used quadratic cover glasses of the borosilicate glass Schott D263M (supplier: Plano GmbH, Germany) with a size of $22 \cdot 22 \cdot 0.21 \mathrm{~mm}^{3}$ to study the stresses induced by irradiation with an ArF excimer laser, and to investigate the possibility of selective deformation and figure correction by these stresses. For good observation of the deformation, thin samples were needed. D263 exhibits a large linear thermal expansion coefficient of $7.2 \cdot 10^{-6} \mathrm{~K}^{-1}$, which leads to large thermal stresses. By my own experience, it shows good ablation characteristics at $193 \mathrm{~nm}$ and has been used in many studies on thin mirror substrates for x-ray telescopes $[22,33,39,82$, 92 94], for which figure correction is a vital method [27]. Due to the manufacturing process by the down-draw method (cf. [95, p. 147]), the surfaces of the sheets are fire polished. The pristine samples usually exhibited a deflection of a few to $15 \mu \mathrm{m}$ over a length of $20 \mathrm{~mm}$. Therefore, according to [96], a compensation for an initially non-planar curvature was not necessary in the application of the Stoney Eq. (1.1).

To study the viscoelastic deformation of fused silica plates by a continuous or structured $\mathrm{SiO}_{x}$ film at temperatures above $1000^{\circ} \mathrm{C}$, I used round substrates of Heraeus Suprasil 1 or 2 (grade A) with a diameter of $25 \mathrm{~mm}$ and a thickness of $1 \mathrm{~mm}$. Suprasil is a common silica glass with a high UV transmission 97 and is 
usually applied in our group for structuring of $\mathrm{SiO}_{x}$ films via backside ablation and production of fused silica phase masks [89]. According to [98], it is a type-III vitreous silica. Therefore, it exhibits a large hydroxyl content of $\leq 1300 \mu \mathrm{g} / \mathrm{g}$, as given by the manufacturer [97]. The thickness of the substrates of $1 \mathrm{~mm}$ was chosen for a larger deformation than in case of thicker substrates. Both sides were mechanically polished by the supplier (Aachener Quarzglas-Technologie Heinrich GmbH \& Co. $\mathrm{KG}$, Germany). Usually, the surface flatness was in the range of a few $100 \mathrm{~nm}$ on a length of $20 \mathrm{~mm}$.

I used cover glasses of the fused quartz material GE124 (supplier: Plano GmbH, Germany) with a size of $22 \cdot 22 \cdot 0.25 \mathrm{~mm}^{3}$ to gain information on the stresses in the system of $\mathrm{SiO}_{x}$ on vitreous silica by observation of the elastic deformation of the cover glasses at room temperature. The cover glasses were chosen because of the low $\mathrm{OH}$ concentration of $5 \mathrm{ppm}$ by weight [99], which leads to a high viscosity at elevated temperatures [100, pp. 14-15], and because of the small thickness, that allows for large deformations. The surface of the samples was fire polished.

\subsection{Thin film deposition}

For this study, films of $\mathrm{SiO}_{x}$ and chromium were deposited by L. J. Richter (IFNano) on vitreous silica and Schott D263M, respectively. He used a Leybold Univex 350 evaporation chamber including an Ar sputtering device and a heated, rotating sample holder.

The substrates were cleaned in an ultrasonic bath in acetone, deionized water and isopropyl alcohol and dry blown with nitrogen. They were mounted on the sample holder by either fixating at two positions at the rim or, in case of round samples, at the whole rim. Before deposition of $\mathrm{SiO}_{x}$ films, the substrates were cleaned insitu by short time $(15 \mathrm{~s})$ application of a radio frequency argon plasma. Pellets of silicon monoxide were thermally evaporated from a joule heated baffled box made from tungsten. During deposition, the residual gas pressure inside the evaporation chamber was typically of the order of $10^{-6} \mathrm{mbar}$, the sample holder was heated to a temperature of about $300^{\circ} \mathrm{C}$ and rotated at a speed of $5 \mathrm{rpm}$. Under these conditions, the deposition rate was manually controlled by variation of the electrical current through the baffled box to a constant value of about $1 \mathrm{~nm} / \mathrm{s}$. The films exhibited a brown color, good absorption in the UV spectral region and reflection spectra close to the ones for pure silicon monoxide. Therefore, it was concluded that the stoichiometry of the films was close to $x \gtrsim 1$.

For deposition of chromium on substrates of Schott D263M, the Cr pellets were heated inside a crucible by an electron beam. The base pressure of the chamber was about $5 \cdot 10^{-6} \mathrm{mbar}$, and the sample holder heated to about $40^{\circ} \mathrm{C}$. To achieve a non-uniform film thickness, the sample holder was not rotated during deposition. The deposition rate was set to a value of $0.8 \mathrm{~nm} / \mathrm{s}$. 


\subsection{Excimer laser processing}

In the course of this work, I applied two different ArF excimer lasers, namely the models Coherent LPX-Pro and Lambda Physik LPX 300, with a wavelength of $193 \mathrm{~nm}$ for surface modification of D263M cover glasses and for ablation of $\mathrm{SiO}_{x}$ films on fused silica substrates. The pulse duration was about $20 \mathrm{~ns}$. The LPX-Pro and the LPX 300 reached maximum pulse energies of about $100 \mathrm{~mJ}$ and $400 \mathrm{~mJ}$, respectively.

For spatial beam shaping, I used simple mask projection setups (Fig. 2.1) or a homogenizer setup (Fig. 2.2). In the mask projection setup, the laser light passes a mask that is imaged with demagnification onto the sample. The masks were chromium on fused silica, like the ones typically used in lithography, or cut-out steel sheets. Due to the almost flat-top output beam profile of the excimer, a to a large degree homogeneous fluence distribution can be obtained in the image plane. For projection, I usually used plano-convex fused silica lenses and a demagnification of $10: 1$. Nominal focal lengths were typically in the range of 50 to $100 \mathrm{~mm}$. The pulse energy was adjusted by the discharge voltage of the excimer laser and a variable attenuator, that was based on a dielectric cut-off filter (cf. [101, Sec. 2.3.3]). For beam guiding, I used dielectric mirrors (cf. [101, Sec. 2.3.2]). Occasionally, to reach high pulse energies, I installed a field lens, which I combined in some cases with an aperture in front of the projection lens to preserve image quality. Fluences of up to $2 \mathrm{~J} / \mathrm{cm}^{2}$ were applied in the image plane. For a line shaped beam profile, I used a slit mask based on two razor blades and a cylindrical projection lens. In that case, the slit and the long axis of the cylindrical lens were oriented parallel to the long axis of the roughly rectangular raw beam of the laser.

In the homogenizer setup (Fig. 2.2), the raw beam was segmented by two crossed arrays of cylinder lenses. Inside the focal plane of a closely installed spherical lens, the light from the segments was superimposed to form a large rectangular spot of uniform fluence distribution (cf. [102, pp. 53-54] or [103]). Because the superposition of the light from the segments can be understood by the Fourier transformation of angles to positions, the lens is called a Fourier lens. The pitch of the cylinder lens arrays was $4 \mathrm{~mm}$ and the focal length $400 \mathrm{~mm}$. The Fourier lens had a nominal focal length of $125 \mathrm{~mm}$.

The samples were attached to a system of three orthogonal linear stages via appropriate mounts. Cover glasses of D263M and GE124 were mounted inside a 3d printed polymer holder with four clamps at the corners of the quadratic samples. Cylindrical samples of Heraeus suprasil were mounted inside a standard optic holder for round optics with $25 \mathrm{~mm}$ diameter.

I measured the output pulse energy of the laser by a Gentec Joulemeter ED500, a pyroelectric sensor, connected to an oscilloscope. Pulse energy behind the projection lens was measured by pyroelectric sensors from Ophir Optronics Solutions Ltd. connected to an Ophir NOVA II display. Division by the area of ablated spots, measured under the light microscope, resulted in the laser fluence at the image plane. 


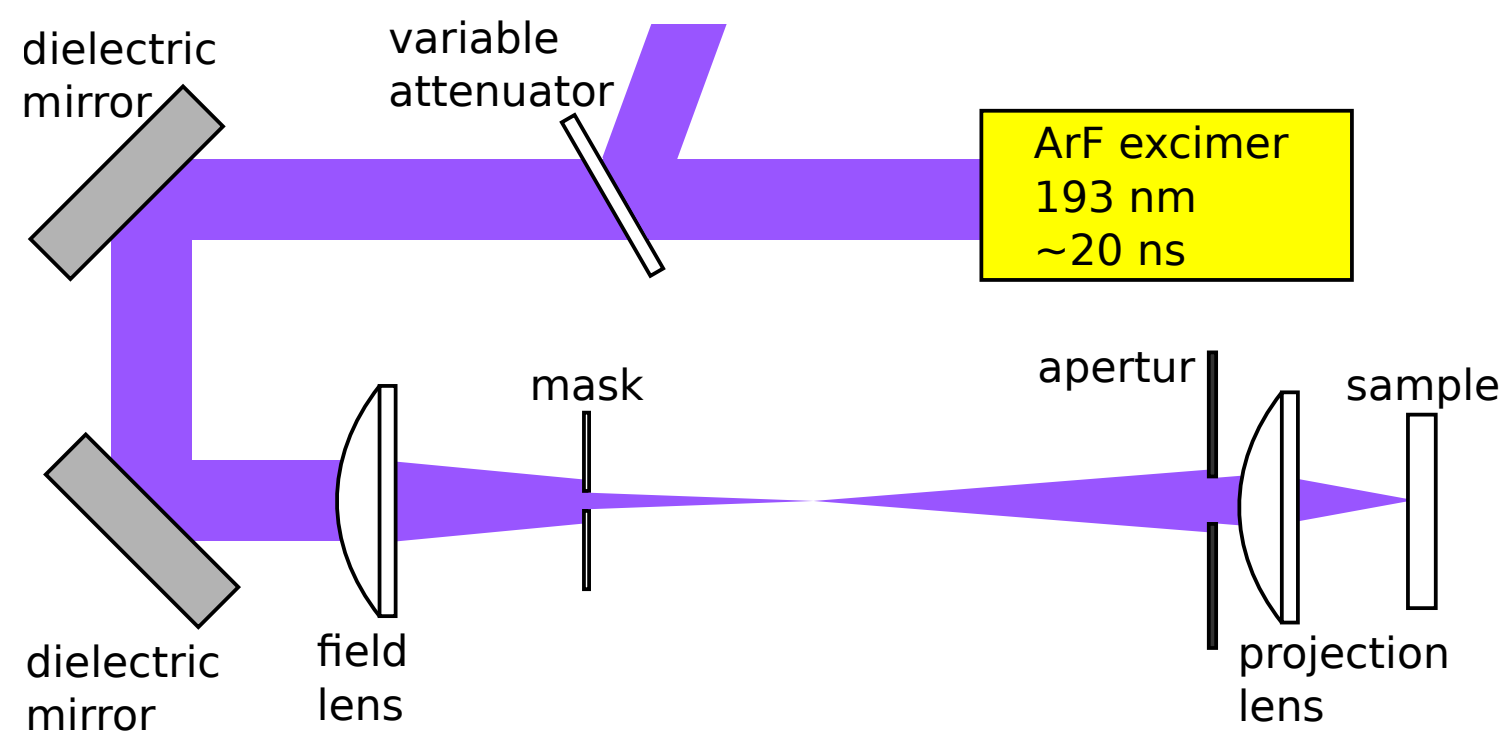

Figure 2.1: A scheme of a typical optical setup used for processing by an ArF excimer laser. A mask is imaged with demagnification onto the sample surface via a projection lens. In case high fluences were needed, a field lens, sometimes together with an aperture in front of the projection lens, was used. The pulse energy was controlled by a variable attenuator or the discharge voltage of the excimer laser. The sample position was adjusted via three linear stages.

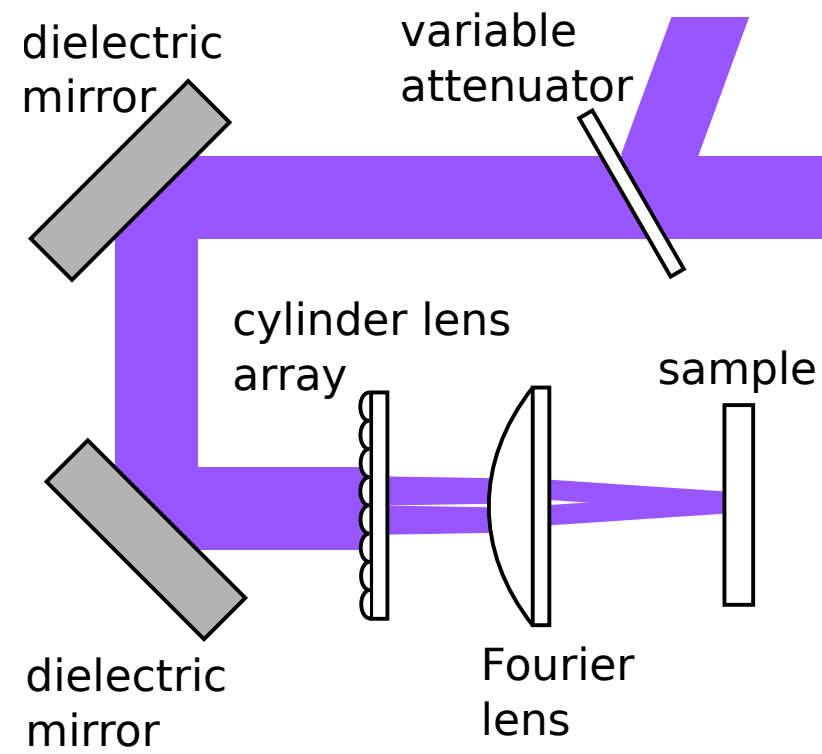

Figure 2.2: In a few cases, a homogenizer setup was used to achieve a large laser spot with a homogeneous fluence distribution. The cylinder lens array segments the laser light and the Fourier lens superposes the light from the segments in its focal plane. The pulse energy was controlled by a variable attenuator or the discharge voltage of the excimer laser. The sample position was adjusted via three linear stages. 


\subsection{Tactile profilometry}

An important part of my experimental work was the measurement of height profiles of surfaces by tactile profilometry. I used short range profiles to determine film thicknesses and the texture of structured surfaces. And I used long range profiles to measure the form and curvature of surfaces.

In tactile profilometry, a tip, called stylus, is drawn across a surface, and the vertical displacement of the stylus is set in relation to its lateral position to achieve a height profile [104, Ch. 6.6.1]. The measured height profile is influenced by the finite radius $r$ of the stylus. For example, a simple estimate for the maximum measureable amplitude $Q_{\max }$ of a sinusoidal surface profile with a period of $d$ is given by 105

$$
Q_{\max }=\frac{d^{2}}{4 \pi^{2} r}
$$

If $d$ is too small, the stylus does not reach the bottom of the pits, and the amplitude of the structure is underestimated by the measurement.

At the beginning of my work, I used a Sloan Dektak 3030 with a diamond stylus of $2.5 \mu \mathrm{m}$ tip radius. Later on, it was replaced by a Bruker Dektak XT-A with a tip radius of $2 \mu \mathrm{m}$. Typical contact forces of the stylus were in the range of 3 (30) to $6 \mathrm{mg}(59 \mu \mathrm{N})$. For form and curvature measurement, I usually used a measurement length of $20 \mathrm{~mm}$ with a measurement duration of $50 \mathrm{~s}$ at a sampling rate of $0.1 \mu \mathrm{m}^{-1}$ for the Dektak 3030 and a duration of $120 \mathrm{~s}$ at a sampling rate of $1.80 \mu \mathrm{m}^{-1}$ for the Dektak XT-A. The calibration of the vertical scale was achieved by measurement of certified step height standards with heights of $905 \mathrm{~nm} \pm 5 \%$ and $(992.2 \pm 5.2) \mathrm{nm}$, respectively. I corrected all measured height profiles for the slope by subtraction of a linear function. I neglected the error induced by this false rotation of the profiles.

It was demonstrated in [106 that the Dektak 3030 can be used for curvature measurements. Anyhow, I verified the form accuracy by measurement of the surface of an optical flat with a flatness of $\lambda / 20$ at $\lambda=632.8 \mathrm{~nm}$, corresponding to an amplitude of $32 \mathrm{~nm}$. Fig. 2.3 shows typical height profiles of the surface of such an optical flat. The specification of the optical flat is met if isolated peaks are neglected. These peaks are usually produced by dirt or surface errors. Therefore, they do not represent the surface form.

To avoid movement of the samples during form measurement, I designed two sample holders ${ }^{1}$ for quadratic samples of $22 \mathrm{~mm}$ edge length and for round samples of $25 \mathrm{~mm}$ diameter, respectively. Fig. 2.4 shows photographs of the holders with mounted samples inside the Dektak XT. The samples rest on three steel balls for defined boundary conditions, and the lateral movement is restricted by steel posts. The measurement directions were always chosen to be in such a direction that the sample movement would be hindered by the posts if they were tagged along by the stylus.

\footnotetext{
${ }^{1}$ The holders were manufactured by Andreas Spata (IFNano).
} 


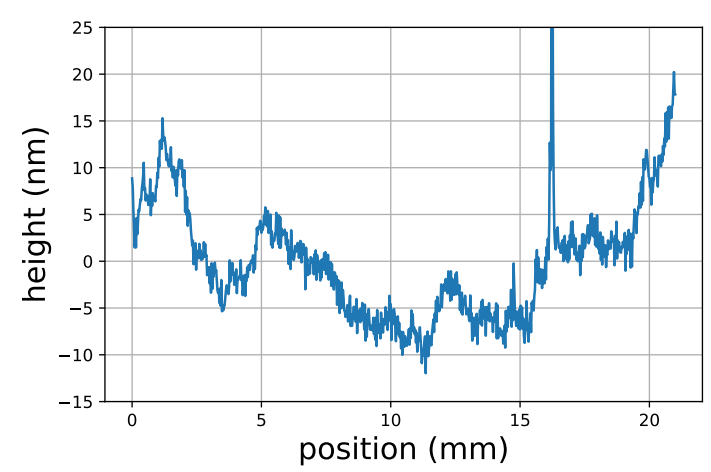

(a) Dektak 3030

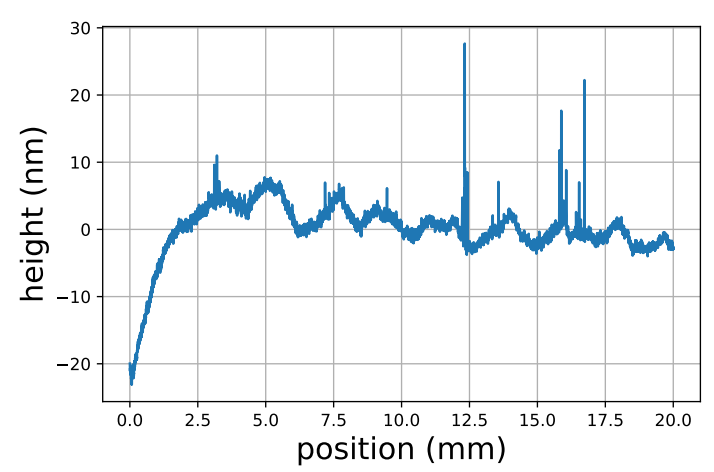

(b) Dektak XT

Figure 2.3: Typical height profiles of a $\lambda / 20$ optical flat measured by (a) the Dektak 3030 and (b) the Dektak XT.

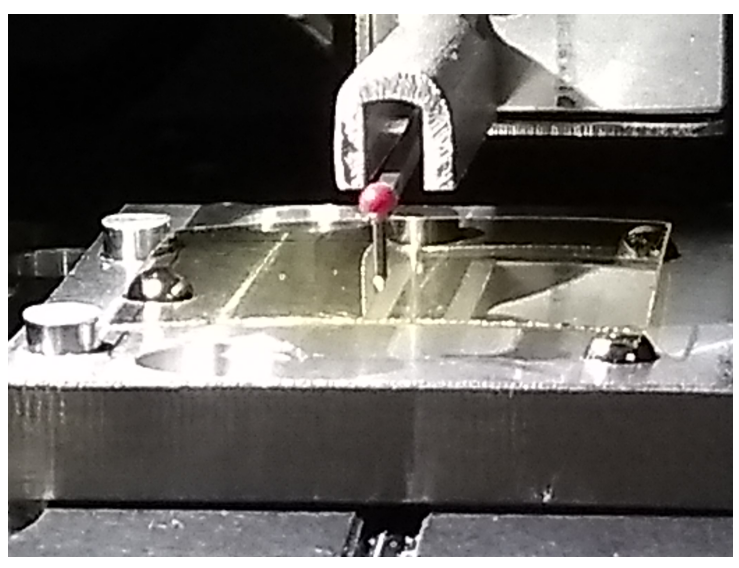

(a) Cover glass

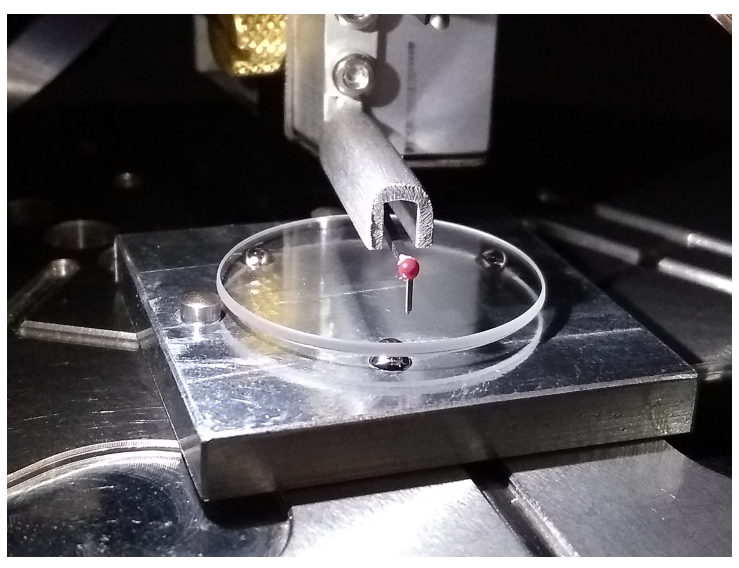

(b) Round plate

Figure 2.4: Photographs of the sample holders used for reproducible form measurement by tactile profilometry. (a) The holder used for quadratic cover glasses of $22 \mathrm{~mm}$ edge length. A strongly deformed GE124 cover glass coated with a $\mathrm{SiO}_{x}$ film is mounted. (b) The holder used for round plates with a diameter of $25 \mathrm{~mm}$. A Suprasil sample is mounted. In both pictures the stylus of the Dektak XT can be seen. 
For form measurement, I usually obtained two height profiles in orthogonal and defined directions. The orientation of the height profiles is sketched in Fig. 2.5. Ideally they cross each other at the center point of the surface. For orientation, I usually marked one edge of the quadratic samples with a diamond scribe. In case of the round samples, I placed a mark at the rim or used a feature of the film for orientation.

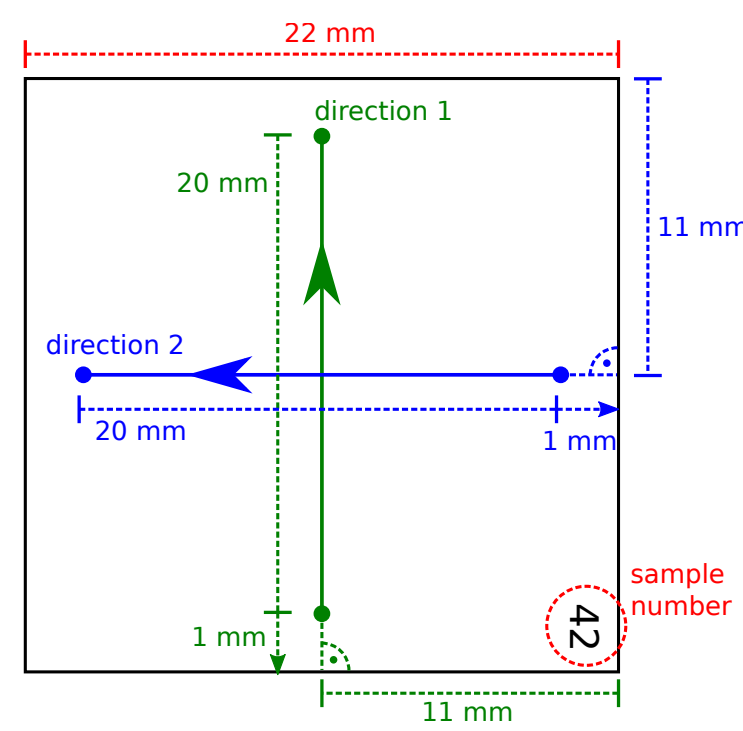

(a)

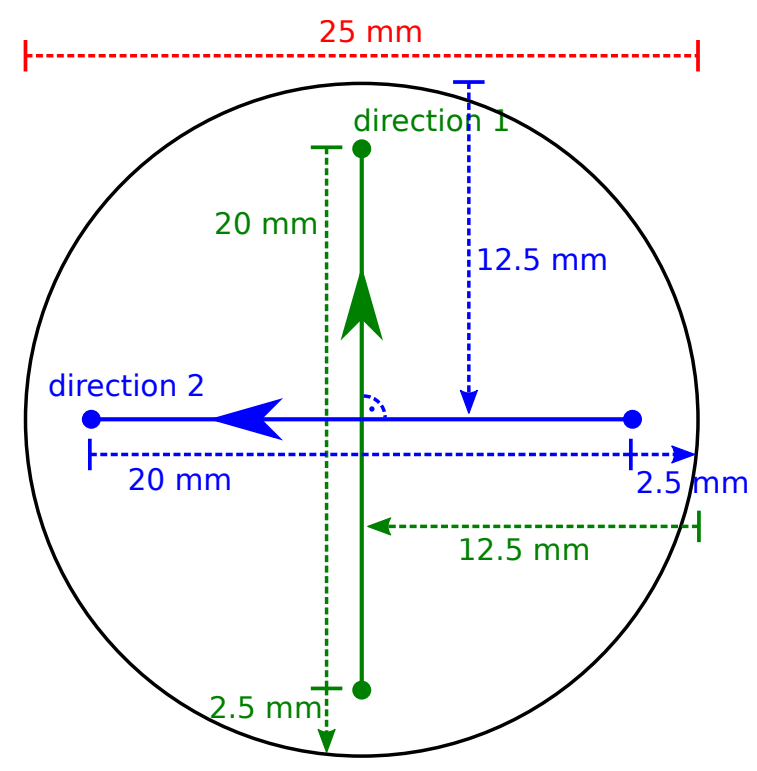

(b)

Figure 2.5: This scheme shows how the measured height profiles were typically positioned on the sample surface of (a) quadratic cover glasses and (b) round plates. (a) The samples were typically marked with their number, which helped in orientation of the samples. (b) For rotational orientation typically a feature of the coating or a mark at the rim were used.

For calculation of the curvature profiles $k(x)$, the measured height profiles $h(x)$ were smoothed by convolution with a Gaussian function by use of the function Gaussian_filter1d of the module Ndimage in SciPy 0.19.1[107] in Python 3. The applied standard deviation was usually on the order of a few $100 \mu \mathrm{m}$. Please note that Gaussian filtering is the standard approach in surface topography measurement for removing non-interesting spatial frequencies from the experimental data 104 , Ch. 8.2.3]. Subsequently, the curvature was calculated via [108, p. 251]

$$
k(x)=\frac{\mathrm{d}^{2} h(x) / \mathrm{d} x^{2}}{\left(1+(\mathrm{d} h(x) / \mathrm{d} x)^{2}\right)^{3 / 2}} .
$$

The derivatives of Eq. 2.2) were calculated by use of the central difference quotient. In many cases I do not state the curvature $k(x)$ but the curvature difference (or curvature change) $\Delta k(x)=k(x)-k_{0}(x)$ with respect to some reference curvature profile $k_{0}(x)$, that, for example, corresponds to the pristine sample.

Gaussian filtering has two major effects on the calculated curvature profiles. First of all, it reduces the noise to a tolerable level. However, there will be some low 
spatial frequency fluctuation left in the curvature profiles. Although in the experimental curvature profiles this noise seems mostly reproducible from measurement to measurement (cf. Fig. 5.1e), it is not for artificial test data (Fig. 2.6). This means, that it is not a systematic artifact of the algorithm but related to the low frequency noise of the instrument. For the sake of simplicity no attempt was made to compensate for it. In contrast, the second major effect of the Gaussian filtering, namely the divergence at the endpoints of the curvature profiles, is indeed an artifact of the algorithm (Fig. 2.6). However, it does not significantly disturb the interpretation of the curvature profiles.

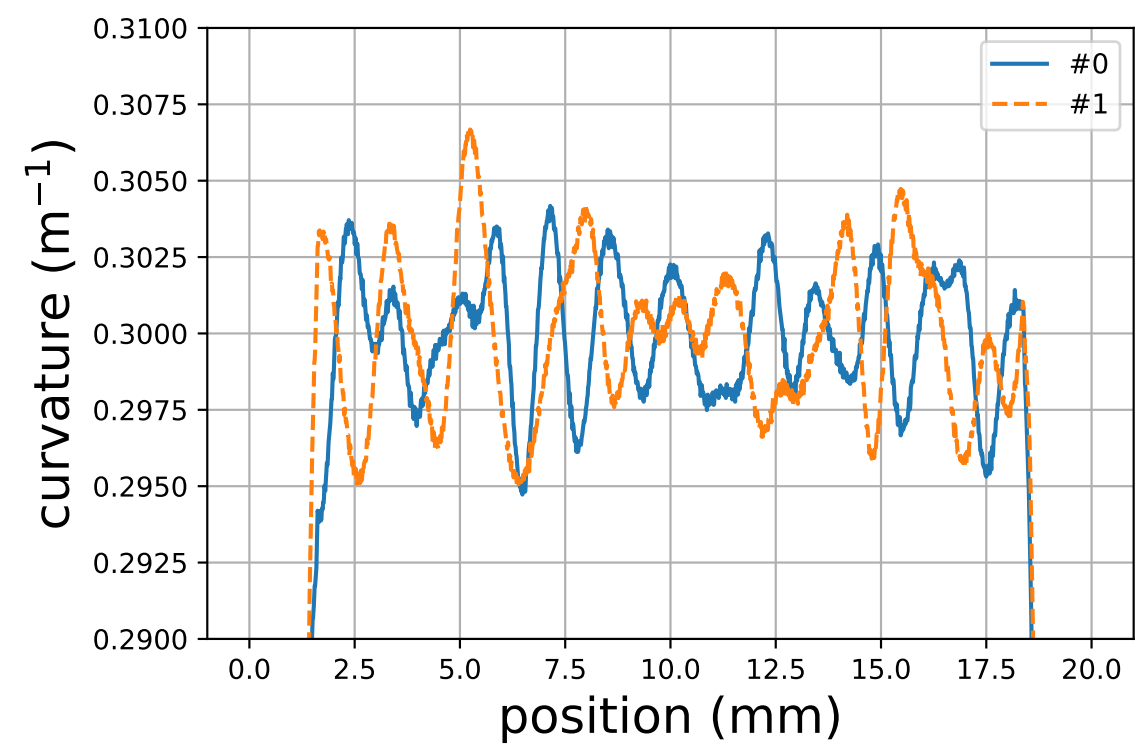

Figure 2.6: Two artificial curvature profiles. For calculation, a parabolic function was blurred by addition of random noise, and the same procedure as for the experimental data was applied. The curvature profiles diverge at the end points and some low frequency spatial noise can be observed.

To estimate the influence of gravity and the stylus on the measured height profiles, the linear elastic sample deformation during measurement was simulated by the finite element method (FEM) (cf. Sec. 2.8.2). By combination of Eq. (6.2.68) with Eq. (3.6.18) of 109 and insertion of the gravitational force $F=\rho_{m} \cdot g$ with mass density $\rho_{m}$ and gravitational acceleration $g$, the dependence

$$
u_{z}^{\max }=\frac{48\left(1-\nu_{s}^{2}\right) g \rho_{m}}{\pi^{6} E_{s}} \frac{l^{4}}{t_{s}^{2}}
$$

for the maximum gravitational deflection of a square plate with simply supported boundary conditions is achieved. In Eq. (2.3), $l$ is the edge length of the plate, $t_{s}$ its thickness, $\nu_{s}$ its Poisson's number and $E_{s}$ its Young's modulus. It can be seen that the displacement increases with the factor $l^{4} / t_{s}^{2}$. For a point load in the middle of the plate surface, the combination of Eq. (6.2.71) and Eq. (3.6.18) of [109] leads to a $l^{2} / t_{s}^{3}$ dependence of the maximum deflection. Therefore, the simulation was only performed for the thinnest samples, namely D263M cover glasses of $210 \mu \mathrm{m}$ thickness. For the boundary conditions, a point bearing like the one of the sample 
holder in Fig. 2.4a, was assumed. The stylus was simulated by a Gaussian pressure distribution with a standard deviation of $2 \mu \mathrm{m}$, that was applied in the middle of the upper surface of the sample. The simulation showed that gravity leads to a maximum displacement of $280 \mathrm{~nm}$, and a stylus with a contact force of $4 \mathrm{mg}$ to a maximum displacement of $8 \mathrm{~nm} \mathrm{~L}^{2}$

For large deformations, the influences of gravity and contact force during measurement were negligible. For small deformations, they canceled out because they were the same at every measurement, and I always considered the change in curvature or height profiles. I estimated the statistical error of the curvature measurement by repeated measurements on the same sample at different days. For the Dektak $\mathrm{XT}$ and samples of D263M, I obtained a standard deviation of the average curvature difference of $0.7 \cdot 10^{-3} \mathrm{~m}^{-1}$ by 15 measurements on a deformed sample. I assumed the same value for cover glasses of GE124. 10 measurements on a deformed plate of Suprasil led to a standard deviation of $2 \cdot 10^{-3} \mathrm{~m}^{-1}$. I obtained the total uncertainty of the average curvature values by combination of the statistical error with the systematic error of the step height standard used for calibration (see above).

To achieve a more complete picture of the sample deformation, I measured topography maps of the surfaces of some samples as well. For this, I applied two different approaches. For the large deformation of Suprasil substrates, I used the internal method of the Dektak XT. This consists of measuring parallel height profiles and placing them side by side. The relative height information of the profiles is preserved to some extend because the stylus tower is not moved up in between the measurements. Fig. 2.7a shows a topography map of the surface of a $\lambda / 20$ optical flat measured by this method. A height deviation in the range of a few $100 \mathrm{~nm}$ can be observed.

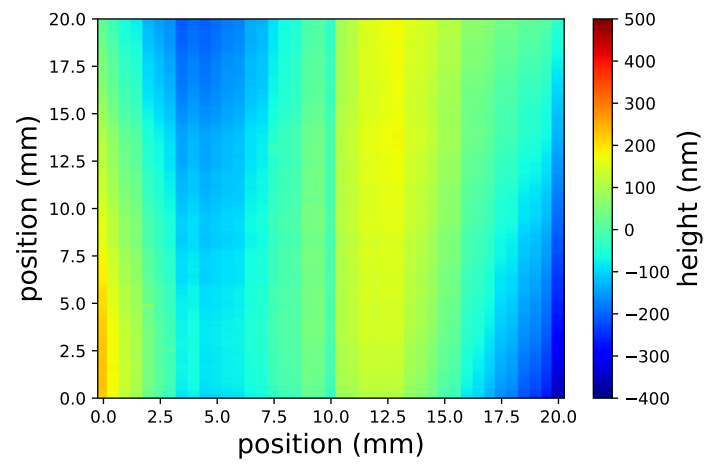

(a)

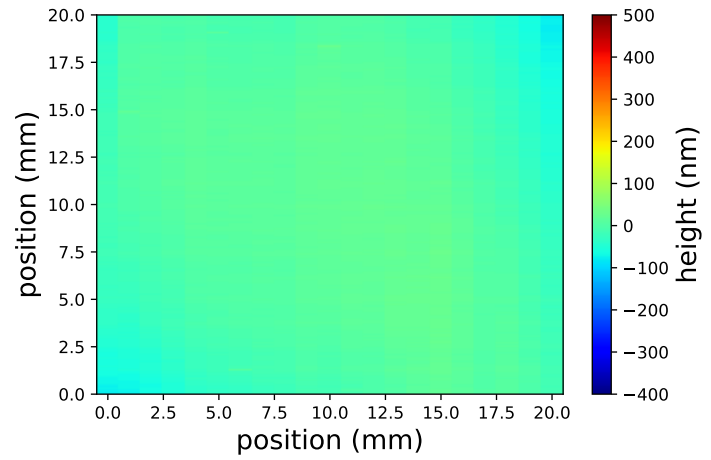

(b)

Figure 2.7: Height maps of the surface of a $\lambda / 20$ optical flat measured by the Dektak XT with two different approaches. (a) Internal method of the device. (b) Measurement of individual height profiles and external assemble.

I used a more precise method for the quantitative analysis of the deformation of D263M samples. In this case, I measured a few individual height profiles in

\footnotetext{
${ }^{2}$ I verified the simulation results by similar simulations for simply supported boundary conditions and comparison with the predictions by Eq. (2.3) and the corresponding equation for deformation by a point load. A good agreement was obtained.
} 
one direction, rotated the sample and the holder by $90^{\circ}$ around the vertical axis and measured another height profile in the orthogonal direction. Afterwards, the relative height information between the parallel profiles was retrieved by the one height profile in orthogonal direction. A height map of the $\lambda / 20$ optical flat measured by this method is plotted in Fig. 2.7b. The flatness of the surface is much better reflected by this method than by the internal method of the Dektak XT. However, please note that both methods do not correct for a deviation in the slope of parallel height profiles. For an even higher precision, I usually obtained topography maps in both measurement directions and combined them. I found that the root-meansquare (RMS) deviation of such height maps obtained from the same sample on different days was about $40 \mathrm{~nm}$. Thus, reproducibility was quite good.

\subsection{Transmission and reflection measurements}

I measured the optical transmission of the samples at normal incidence via a Perkin Elmer Lambda $19 \mathrm{UV} / \mathrm{VIS} / \mathrm{NIR}$ spectrometer usually in the wavelength range of 190 to $860 \mathrm{~nm}$ at a step width of $1 \mathrm{~nm}$ and a slit width corresponding to a resolution of $2 \mathrm{~nm}$. For determination of film thicknesses, I also measured optical reflection spectra of the coated side of samples. In the beginning of my work, I also used the Lambda 19 for this purpose by application of a reflection sample holder. In that, a polished fused silica sample served as a reference.

Later on, we bought a reflectometer (Filmetrics F20UV), that is especially designed for optical film thickness measurement. It delivers the light of a deuterium and a halogen lamp by one branch of a Y-fiber and collects the reflected light by the other branch. The reflected light is analyzed by a CCD spectrometer. For reference I used silicon and Schott BK7 samples delivered by the manufacturer of the reflectometer. To evaluate the thickness of $\mathrm{SiO}_{2}$ layers grown on $\mathrm{SiO}_{x}$ and $\mathrm{Si}$ samples, I fitted the obtained reflection spectra by the software of the F20UV via adjustment of the film thickness of the prior defined layers. For the $\mathrm{SiO}_{2}$ material, I used the optical properties of the internal database of the software. For $\mathrm{SiO}_{x}$, the internal optical properties of $\mathrm{SiO}$ worked also fine although a small overestimation of the film thickness compared to the thickness determined by measurement of a film step was observed. This was accepted because in most cases I was interested in the thickness of the $\mathrm{SiO}_{2}$ layer.

\subsection{Annealing}

I annealed samples in a muffle furnace in air or in a tube furnace in oxygen, nitrogen or argon flow. The muffle furnace was a Nabertherm L5/13/B410. The furnace chamber had a volume of $5 \mathrm{l}$ and was heated from both sides by four filament coils on each side. Temperature was controlled by a thermocouple type $\mathrm{S}$, leading to a measurement uncertainty of $\pm 1 \mathrm{~K}$. Typical heating and cooling curves for a processing temperature of $1120^{\circ} \mathrm{C}$ are plotted in Fig. 2.8. Usually, I programmed a linear 
temperature increase with time for heating. No active cooling was applied. Inside

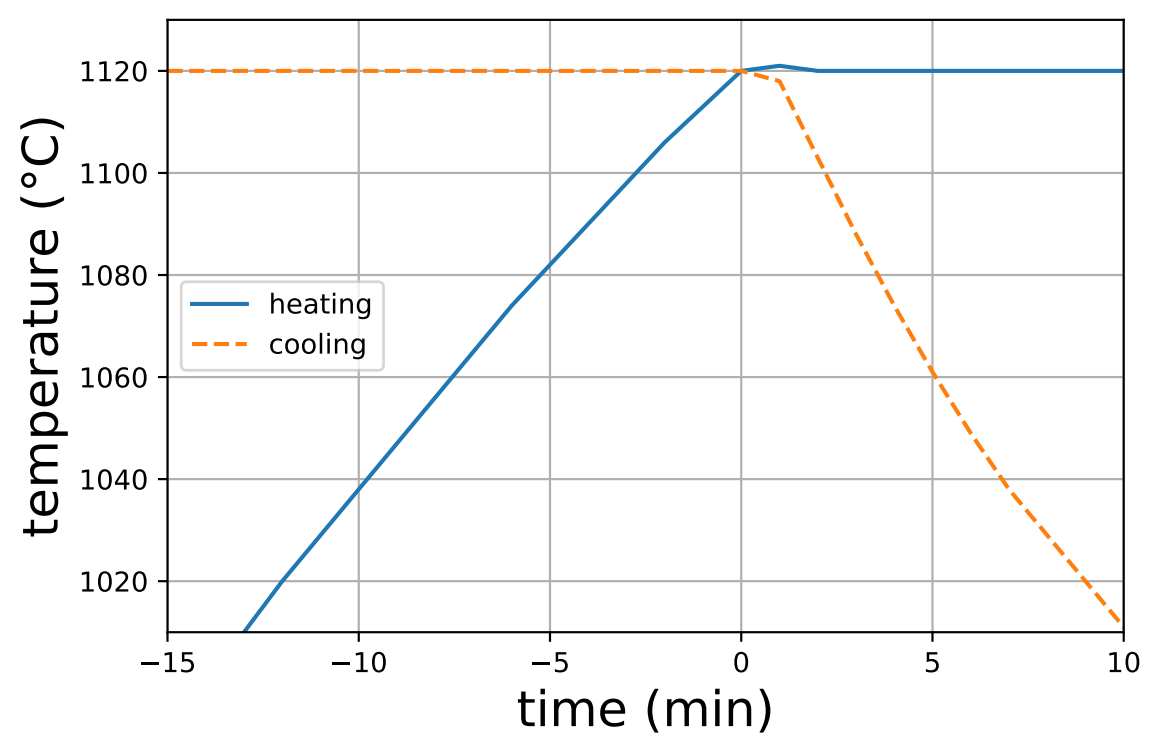

Figure 2.8: Typical heating and cooling curves of the Nabertherm L5/13/B410 muffle furnace for a processing temperature of $1120^{\circ} \mathrm{C}$. For heating, a linear temperature increase was set. Cooling happened passively via natural heat loss.

the muffle furnace, samples of Heraeus Suprasil were laid on a ground fused silica plate of $4 \mathrm{~mm}$ thickness (horizontal orientation, Fig. 2.9a) or oriented vertically (Fig. $2.9 \mathrm{~b}$ by clamping between two fused silica rods 3 . In both cases, they were placed inside a ceramic box covered by a ceramic plate in case of horizontal annealing.

For annealing in determined atmospheres, I applied a tube furnace (Nabertherm R50/250/13). Fig. 2.10 shows a scheme of the experimental setup. The sample is placed inside a fused silica tube of $38 \mathrm{~mm}$ outer diameter, $2.4 \mathrm{~mm}$ wall thickness and $\approx 700 \mathrm{~mm}$ length. Thus, the inner volume of the tube is about $0.6 \mathrm{l}$. Also in this case, I usually placed the samples on a ground fused silica underlay of 3 to $4 \mathrm{~mm}$ thickness. The fused silica tube lies inside another tube of Sillimantin, an alumina based ceramic material, with an outer diameter of 50 and an inner diameter of $40 \mathrm{~mm}$. The oven is heated by a filament coil wrapped around the Sillimantin tube, and the temperature is controlled via a thermocouple type $\mathrm{S}$ placed beneath the Sillimantin tube. A steady flow of oxygen (purity $\geq 99.95 \%$ ), nitrogen $(\geq 99.999 \%$ ) or $\arg$ on $(\geq 99.998 \%)$ can be realized by two variable area flowmeters (rotameters). Typical flow rates were between 50 and $100 \mathrm{l} / \mathrm{h}$. I found that, even without gas flow, the actual temperature inside the inner tube deviated significantly from the temperature measured by the internal thermocouple. Therefore, for a more precise temperature measurement, I introduced a type $\mathrm{K}$ thermocouple from the gas outlet of the fused silica tube. For annealing at a set temperature of $1120^{\circ} \mathrm{C}$ (actually $1060(10)^{\circ} \mathrm{C}$ ), I usually used a heating rate of about $18 \mathrm{~K} / \mathrm{min}$. Cooling happened via natural heat loss.

I checked the quality of the inert atmospheres inside the tube furnace by anneal-

\footnotetext{
${ }^{3}$ This way of vertical orientation was first performed by Lukas Janos Richter (IFNano).
} 


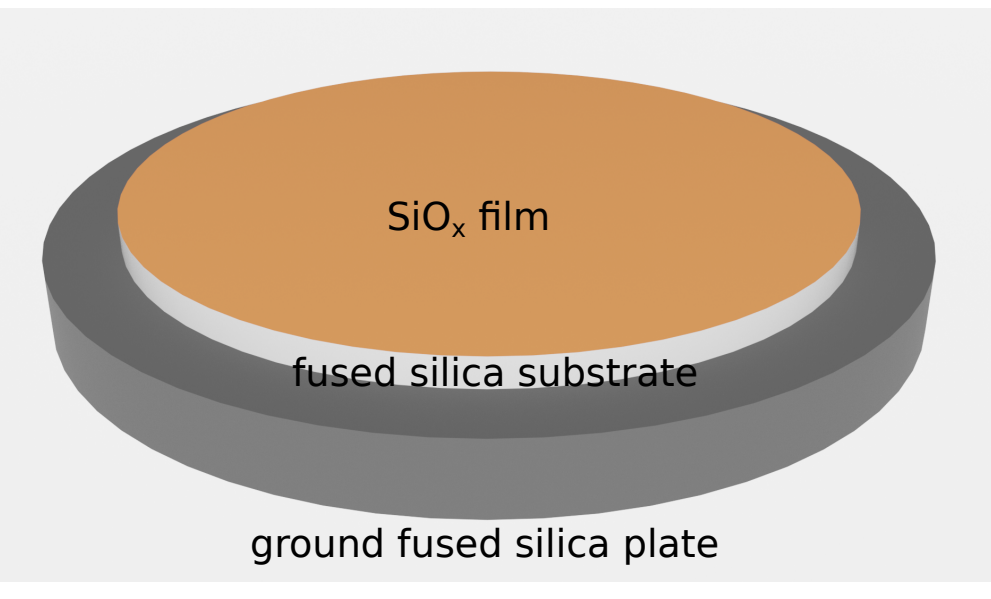

(a)

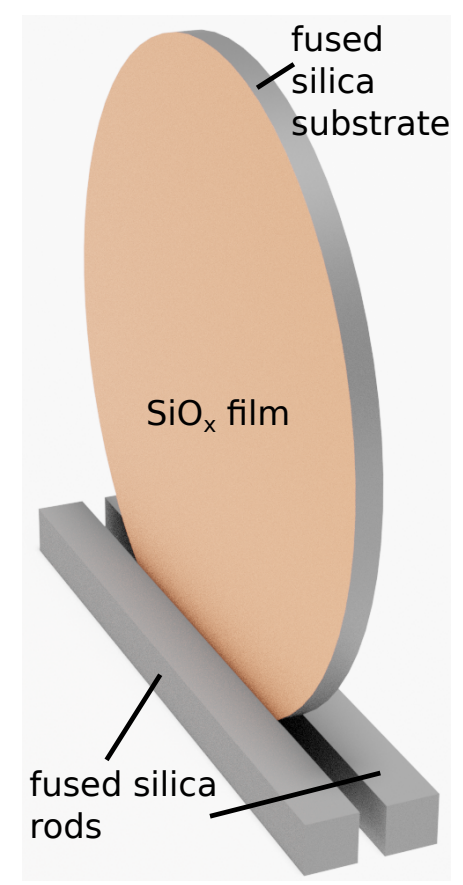

(b)

Figure 2.9: Coated fused silica samples were annealed in the muffle furnace in ambient atmosphere in horizontal (a) or vertical orientation (b). For the former, the sample was placed on a ground fused silica plate with a diameter of $28 \mathrm{~mm}$ and a thickness of $4 \mathrm{~mm}$. For the latter, the sample was clamped between two rods of fused silica.

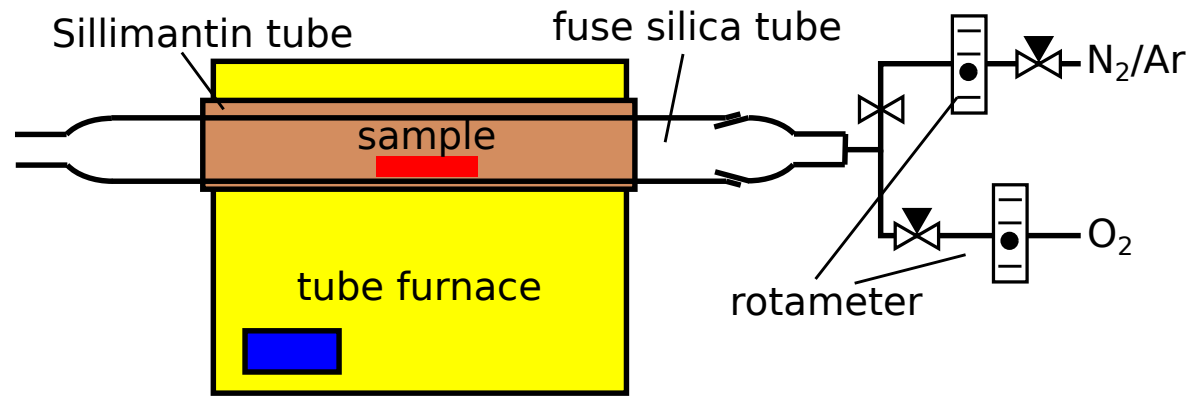

Figure 2.10: Scheme of the experimental setup at the tube furnace for annealing in different atmospheres. 
ing of pristine silicon samples and subsequent measurement of the oxide thickness. For annealing at $1060(10)^{\circ} \mathrm{C}$ for a duration of $5 \mathrm{~h}$, an oxide thickness of about $20 \mathrm{~nm}$ grew in nitrogen flow, while oxygen flow led to a thickness of about $312 \mathrm{~nm}$. This shows that oxygen and water concentration was effectively reduced, but still some oxidation was to be expected for annealing of $\mathrm{SiO}_{x}$ films.

\subsection{Etching experiments}

To remove surface material from the samples, I applied different solutions of potassium hydroxide. I used aqueous solutions of different concentrations of Deconex 15 pf-x (Borer Chemie), a KOH based detergent, in combination with an ultrasonic cleaner for simple removal of debris (small particles generated during laser ablation) and reduction of laser generated stresses. I also applied a $5 \mathrm{wt} \%$ solution of $\mathrm{KOH}$ for systematic removal of the laser generated stresses during a $90 \mathrm{~min}$ treatment inside an ultrasonic bath (EMAG Emmi-08 ST) at a temperature of about $80^{\circ} \mathrm{C}$.

For a better reproducibility of the etch rate, I applied a precisely mixed 1.000(2) mol/l $(\approx 5.3 \mathrm{wt} \%$, supplier: Carl Roth) aqueous $\mathrm{KOH}$ solution inside a PTFE beaker of $25 \mathrm{ml}$ volume, in which the samples were put. The beaker was placed inside an ultrasonic bath (Elmasonic P) for precise temperature control, and covered to avoid losses due to evaporation. I used a temperature of $80^{\circ} \mathrm{C}$ and controlled the etching duration with a stopwatch. No ultrasonification was applied. The maximum duration of one etching step was 15 min and the $\mathrm{KOH}$ solution was renewed and reheated before each etching step. I estimated that the change in concentration due to the consumption of $\mathrm{OH}^{-}$ions during such an etching step is below $1 \cdot 10^{-3} \mathrm{~mol} / \mathrm{l}$.

\subsection{Finite element simulations}

\subsubsection{Introduction to the finite element method and the FEniCS project}

The finite element method (FEM) is a numerical method for solving partial differential equations on a certain domain $\Omega$ [110, 111]. It was used in this study to simulate the linear elastic and linear viscoelastic deformations of the samples and to gain a better understanding of the experimental results by comparison with the simulation results.

The FEM is based on variational methods (weighted residuals or Rayleigh-Ritz) and the discretization of the function that is used for approximation of the solution [112, Ch. 8]. The method of weighted residuals is used to convert a partial differential equation into a system of ordinary differential equations. Consider the equation

$$
\mathcal{D} u(\mathbf{x}, t)=f(\mathbf{x}, t)
$$


for the scalar field variable $u(\mathbf{x}, t)$ and the differential operator $\mathcal{D}$. For a good approximation $\tilde{u}(\mathbf{x}, t)$ of $u(\mathbf{x}, t)$, the residual

$$
R(\mathbf{x}, t)=\mathcal{D} \tilde{u}(\mathbf{x}, t)-f(\mathbf{x}, t)
$$

should be small. For this, the expansion

$$
u(\mathbf{x}, t) \approx \sum_{i} a_{i}(t) \phi_{i}(\mathbf{x})
$$

is made, in which $\phi_{i}(\mathbf{x})$ is a complete set of basis functions and the $a_{i}(t)$ are some weights. By demanding that $R(\mathbf{x}, t)$ is small, the $a_{i}(t)$ can be found. However, there exist different measures, like collocation, least-squares or Galerkin, for the 'size' of $R(\mathbf{x}, t)$. In the Galerkin method,

$$
\int R(\mathbf{x}) w_{i}(\mathbf{x}) \mathrm{d} \mathbf{x}=0
$$

is set, where $w_{i}$ are a family of nontrivially related weighting functions that are usually chosen to be the same as the $\phi_{i}$.

In the FEM, the $\phi_{i}=w_{i}$ are also chosen such that they are nonzero only on small regions of the domain, called the elements, and such that the $a_{i}(t)$ adopt the values of $u\left(\mathbf{x}_{i}, t\right)$ at certain points (nodes) $x_{i}$ [112, Ch. 8]. These conditions can be fulfilled if the $\phi_{i}$ are polynomials that are zero at all but one node. Such functions are the Lagrange interpolation functions. The points (or nodes) are created by division (and usually also approximation) of the domain $\Omega$ into a set of primitive geometrical elements, like e. g. tetrahedra for a three dimensional domain (Fig. 2.11). This, so called meshing, is an elementary step of a FE analysis.

If the interpolation functions are applied in Eq. 2.7), a set of ordinary differential equations is obtained, which, in matrix form, involves sparse matrices [112, Ch. 8]. Solving of such matrices is another important step of a FE analysis. Via partial integration, the order of the highest derivative in Eq. (2.7) can be reduced and the differentiation partially shifted on the weight functions. The obtained equation is called the weak form because it poses weaker requirements on the $\phi_{i}$ [110.

For the main part of the FE analysis, I used the FEniCS platform [113, 114. It is an open-source platform for solving partial differential equations by the FEM, which was developed by scientists and programmers. It exhibits a Python interface, which I used. For me, the advantage of the FEniCS platform was that it does mostly all the algebra by itself. Basically, the user has to implement the physics in form of the weak form of the partial differential equation, the mesh and the boundary conditions. Further, the user needs to specify the kind of interpolation functions. I also changed the solver for the assembled equations (the set of ordinary differential equations) and the numerical tolerance, in order to obtain a good solution. I used FEniCS 2018.1.0 (dolfin-version) on a Linux subsystem on Windows 10.

I used polynomial interpolation functions of first or mostly second order. Depending on the problem, I applied different solvers and preconditioners. For small problems, I used the built in Gaussian elimination method, which ran into memory 
errors for larger problems. Therefore, I switched to the MUMPS solver, which is a direct method, or to the conjugate gradient method.

For the generation of meshes, I used the open-source finite element mesh generator Gmsh 4.3.0 [115] on the operation system Windows 10. The meshes were saved in the .msh format (file version 2) and converted to .xml files via DOLFIN [116], a component of the FEniCS platform. A typical mesh is shown in Fig. 2.11. I always used tetrahedral elements, with sizes of roughly $100 \mu \mathrm{m}$ to $1 \mathrm{~mm}$ in the lateral dimension. For thin films, the mesh size in the direction normal to the surface was usually the same as the film thickness. Although this led to very small angles inside the corners of the tetrahedra, a good simulation accuracy was still achieved.

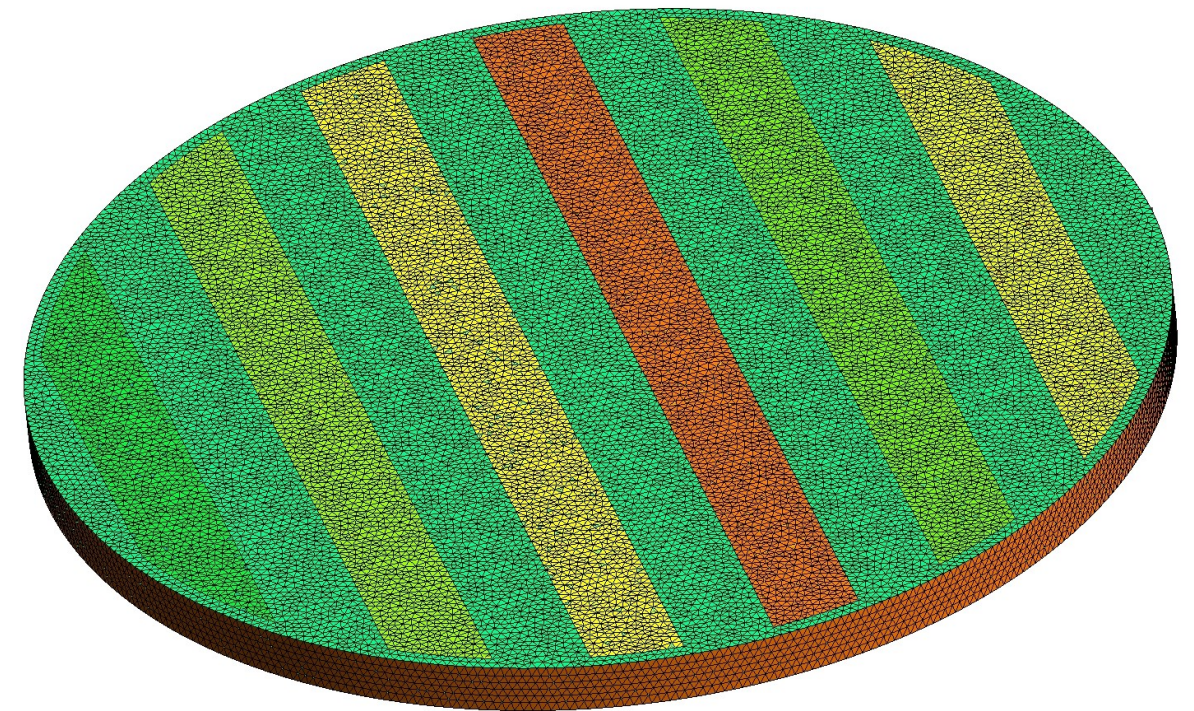

Figure 2.11: A mesh for simulation of the deformation of a $25 \mathrm{~mm}$ diameter plate with a line structured film, as viewed in Gmsh. The lines exhibit different colors because I created them as individual objects, which I unified with the substrate. The mesh size in the lateral direction is $200 \mu \mathrm{m}$.

\subsubsection{The linear elastic simulation}

I wrote a three dimensional linear elastic simulation for comparison with the experimentally measured deformations. For this, I assumed that the mechanical behavior of the materials is isotropic and homogeneous. The deformation is described by the displacement field $\mathbf{u}$, that depends on the sample geometry, the boundary conditions and the material parameters. The underlying differential equations are the Lamé-Navier equations [40, Eq. (7.2.17)]

$$
\mu \nabla^{2} \mathbf{u}+(\mu+\lambda) \nabla(\nabla \cdot \mathbf{u})+\rho_{m} \mathbf{f}=\rho_{m} \frac{\partial^{2} \mathbf{u}}{\partial t^{2}}
$$

where $\mu=G=E /(2(1+\nu))$ and $\lambda=\nu E /((1+\nu)(1-2 \nu))$ are the Lamé constants, $\mathbf{f}$ is the body force vector and $\rho_{m}$ is the mass density. I only considered the static case $\frac{\partial^{2} \mathbf{u}}{\partial t^{2}}=0$. On the boundary of the domain, either the displacement field $\mathbf{u}$ or the 
traction $\mathbf{T}=\hat{\mathbf{n}} \cdot \boldsymbol{\sigma}$ is specified, where $\hat{\mathbf{n}}$ is the unit normal vector to the boundary surface and $\boldsymbol{\sigma}$ is the stress tensor [40, Sec. 7.2.2.4].

The weak formulation of Eq. (2.8) for the static case is [117]

$$
\int_{\Omega} \boldsymbol{\sigma}(\mathbf{u}): \boldsymbol{\epsilon}(\mathbf{w}) \mathrm{d} x-\int_{\Omega} \mathbf{f} \cdot \mathbf{w} \mathrm{d} x-\int_{\partial \Omega_{T}} \mathbf{T} \cdot \mathbf{w} \mathrm{d} A=0
$$

with

$$
\begin{aligned}
& \boldsymbol{\sigma}(\mathbf{u})=\lambda(\nabla \cdot \mathbf{u}) \mathbf{1}+2 \mu \boldsymbol{\epsilon}(\mathbf{u})-\boldsymbol{\Sigma}_{0}, \\
& \boldsymbol{\epsilon}(\mathbf{w})=\frac{1}{2}\left(\nabla \mathbf{w}+(\nabla \mathbf{w})^{\top}\right),
\end{aligned}
$$

$\boldsymbol{\epsilon}$ being the strain tensor, w a test function (a weight function), $\partial \Omega_{T}$ being the fraction of the boundary on which the traction $\mathbf{T}$ is specified and $\mathbf{1}$ the unity matrix. The term $\boldsymbol{\sigma}: \boldsymbol{\epsilon}=\sum_{i, j} \sigma_{i j} \epsilon_{i j}$ is the double-dot product (cf. [40, Sec. 2.5.2]). Please note that I added the tensor $\boldsymbol{\Sigma}_{0}$ to Eq. (2.10) to account for any prestress inside the materials.

To define different material parameters in different domains, I used the Expression class in combination with $\mathrm{C}++$ strings, as is demonstrated in [117, Sec. 4.3.1]. I tested the simulation by comparison of the results for a continuous film with the predictions of the Stoney Eq. (1.1). A good agreement was achieved. Further, I obtained faith in the simulation by the good agreement of measurement and simulation results for the case of laser induced stresses (cf. Figs. 3.4, 3.5, 3.21).

\subsubsection{The linear viscoelastic simulation}

An elastic deformation is instantaneous (movement at the speed of sound), recoverable [100, p. 371] and for small displacements (linear elasticity) there is a linear relation between stress and strain [40, Sec. 6.3.1]. Thus, the material behaves like an ideal spring with the stress strain relation $\sigma=k_{\text {spring }} \epsilon$ in one dimension and the spring constant $k_{\text {spring }}$ 40, Sec. 9.2.2]. In contrast, the viscous deformation is a nonrecoverable flow of material 100 , p. 371]. This can be idealized by a dashpot with the stress strain relation $\sigma=\eta \mathrm{d} \epsilon / \mathrm{d} t$ with viscosity $\eta[40$, Sec. 9.2.2]. Therefore, to model viscoelastic behavior, usually a combination of spring and dashpot elements is considered [118, Ch. 2]. Inside this thesis, I will only deal with the Maxwell model [40, Sec. 9.2.2], which is a serial combination of one spring and one dashpot (Fig. 2.12 and therefore is the most simple description of a viscoelastic fluid. For a constant stress $\sigma_{0}$ applied at time $t=0$ (creep response), the strain

$$
\epsilon(t)=\frac{\sigma_{0}}{k_{\text {spring }}}\left(1+\frac{t}{\tau}\right)
$$

evolves linear with time. Here, $\tau=\eta / k_{\text {spring }}$ is the relaxation time. The other way round, for a constant strain $\epsilon_{0}$ applied at $t=0$ (relaxation response), the stress

$$
\sigma(t)=\epsilon_{0} k_{\text {spring }} \exp \left(-\frac{t}{\tau}\right)
$$




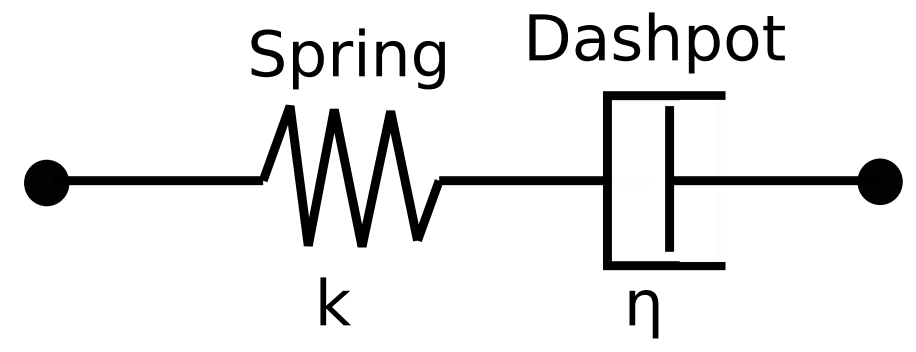

Figure 2.12: The maxwell element consists of a spring and a dashpot in series and describes the behavior of a simple viscoelastic fluid.

relaxes exponentially with time.

In general, a much more complicated combination of springs and dashpots in serial or parallel can be used for description of a viscoelastic material [40, Sec. 9.2]. For an isotropic linear viscoelastic material the stress-strain relations take the form [40, Eq. (9.3.19)]

$\sigma_{i j}(t)=2 \int_{-\infty}^{t} G\left(t-t^{\prime}\right) \frac{\mathrm{d} \epsilon_{i j}\left(t^{\prime}\right)}{\mathrm{d} t^{\prime}} \mathrm{d} t^{\prime}+\delta_{i j} \sum_{k} \int_{-\infty}^{t}\left[K\left(t-t^{\prime}\right)-\frac{2}{3} G\left(t-t^{\prime}\right)\right] \frac{\mathrm{d} \epsilon_{k k}\left(t^{\prime}\right)}{\mathrm{d} t^{\prime}} \mathrm{d} t^{\prime}$,

where $\delta_{i j}$ is the Kronecker delta. Please note that the stress at time $t$ depends on the strain $\epsilon\left(t^{\prime}\right)$ at all times $t^{\prime}<t$. The material is now described by the bulk modulus $K$ and shear modulus $G$ (instead of the Young's modulus $E$ and Poisson's ratio $\nu$ ), which represent the dilatational (volume changing or hydrostatic) and deviatoric (shape changing) deformations, respectively [118, Ch. 2]. For the simulation, I assumed that the dilatational deformation is purely elastic, i. e. $K(t)=$ const., which is a good approximation, as long as the sample is only constrained along two axes [118, Sec. 7.7]. And I assumed that the deviatoric deformation is described by the Maxwell model, i. e. $G(t)=G_{0} \exp (-t / \tau)$ with relaxation time $\tau=\eta / G_{0}$ and shear viscosity $\eta$.

In analogy to Eq. (2.9), the weak form for the elastic problem, the weak form of the current viscoelastic problem can be written as 1119,120

$$
I_{A}(\mathbf{u}(t), \mathbf{w})-\int_{0}^{t} I_{B}(t, s, \mathbf{u}(s), \mathbf{w}) \mathrm{d} s-L(t, \mathbf{w})=0
$$

with

$$
\begin{aligned}
I_{A}(\mathbf{u}, \mathbf{w}) & =\int_{\Omega} \sigma_{A}(\mathbf{u}): \epsilon(\mathbf{w}) \mathrm{d} x \\
I_{B}(t, s, \mathbf{u}, \mathbf{w}) & =\int_{\Omega} \sigma_{B}(t, s, \mathbf{u}): \epsilon(\mathbf{w}) \mathrm{d} x \\
L(t, \mathbf{w}) & =\int_{\Omega} \mathbf{f}(t) \cdot \mathbf{w} \mathrm{d} x+\int_{\partial \Omega_{T}} \mathbf{T}(t) \cdot \mathbf{w} \mathrm{d} A
\end{aligned}
$$


and

$$
\begin{aligned}
\sigma_{A}(\mathbf{u}) & =\left(K-\frac{2}{3} G(0)\right) \nabla \cdot \mathbf{u} \cdot \mathbf{1}+2 G(0) \epsilon(\mathbf{u}) \\
\sigma_{B}(t, s, \mathbf{u}) & =\frac{2}{\tau} G(t-s)\left(-\frac{1}{3} \nabla \cdot \mathbf{u} \cdot \mathbf{1}+\epsilon(\mathbf{u})\right) .
\end{aligned}
$$

The time integral in Eq. (2.15) is solved numerically between time steps $t_{0}$ and $t_{i}$ by the trapezoidal rule (cf. [121, Sec. 6.2]) with time interval $\Delta t$ :

$$
\begin{aligned}
& \int_{0}^{t_{i}} I_{B}\left(t_{i}, s, \mathbf{u}(s), \mathbf{w}\right) \mathrm{d} s \\
& =\Delta t\left[\frac{1}{2} I_{B}\left(t_{i}, t_{0}, \mathbf{u}_{0}, \mathbf{w}\right)+\sum_{j=1}^{i-1} I_{B}\left(t_{i}, t_{j}, \mathbf{u}_{j}, \mathbf{w}\right)+\frac{1}{2} I_{B}\left(t_{i}, t_{i}, \mathbf{u}_{i}, \mathbf{w}\right)\right]
\end{aligned}
$$

for $i \geq 2$.

Unfortunately, the second summand (the sum term) on the right hand side of Eq. 2.21) depends on all prior solutions $\mathbf{u}_{j}$ and therefore is a potential bottleneck regarding simulation speed. A solution to this problem is given in [122, Sec. 5.3] by rewriting into a recursive formula:

$$
\sum_{j=1}^{i-1} I_{B}\left(t_{i}, t_{j}, \mathbf{u}_{j}, \mathbf{w}\right)=\exp \left(-\frac{t_{i}-t_{i-1}}{\tau}\right) \sum_{j=1}^{i-2} I_{B}\left(t_{i-1}, t_{j}, \mathbf{u}_{j}, \mathbf{w}\right)+I_{B}\left(t_{i}, t_{i-1}, \mathbf{u}_{i-1}, \mathbf{w}\right) .
$$

Now, the sum term can be calculated at every time $t_{i}$ from the value at time $t_{i-1}$ and from the prior solution $\mathbf{u}_{i-1}$. Any prestresses were implemented as in the elastic case in Eq. 2.10 by addition of a stress tensor $\boldsymbol{\Sigma}_{0}$ to Eq. (2.19).

I extensively tested the code by comparison to the results of simple linear viscoelastic calculations for the deformation of a cube under different boundary conditions. I calculated the analytic solutions by the viscoelastic analogy, which I describe in Sec.4.1. For the case of a continuous elastic film on a linear viscoelastic substrate, I also obtained a good agreement with linear viscoelastic solutions I derived in Sec. 4.1.1 and 4.1.2, if sufficiently small time intervals $\Delta t$ were used (cf. Fig. 4.4). 


\section{Chapter 3}

\section{Elastic deformation of borosilicate glass plates by laser induced stresses}

\subsection{Experimental results}

I found that cover glasses of Schott D263M can be bent significantly by irradiation with the light of an ArF excimer laser with a wavelength of $193 \mathrm{~nm}$ and a pulse duration of about $20 \mathrm{~ns}$. I studied this effect in detail and here I report on the results. We already published many of the results in compressed form in [123].

\subsubsection{Example for deformation due to irradiation}

A typical irradiation pattern used in this study is shown in Fig. 3.1 as a light microscope image of the surface of a cover glass of D263M after irradiation. The quadratic laser spot, generated by projection of a quadratic mask and a demagnification by a factor of ten, had an edge length of $200 \mu \mathrm{m}$. The sample was scanned with a step width of $220 \mu \mathrm{m}$ in both lateral directions. At each position, one laser pulse with a fluence of $2.0(1) \mathrm{J} / \mathrm{cm}^{2}$ was used in this case. The irradiated area is sharply bounded and homogeneous in appearance, indicating a flat top beam profile in the image plane of the optical setup.

An example for the deformation of a D263M cover glass by irradiation is shown in Fig. 3.2. The surface of one side of the sample has been fully irradiated by a pattern as shown in Fig. 3.1. The laser fluence was $360(30) \mathrm{mJ} / \mathrm{cm}^{2}$. Fig. $3.2 \mathrm{a}$ and Fig. $3.2 \mathrm{~b}$ show the height profiles of the non-irradiated side of the sample, which are oriented as indicated in Fig. 2.5a. The profiles of the pristine sample exhibit a concave shape with an amplitude in the range of 1 to $16 \mu \mathrm{m}$. By irradiation, the profiles become relatively more convex (less concave). After a $100 \mathrm{~min}$ etching process in a $5 \mathrm{wt} \% \mathrm{KOH}$ solution at about $80^{\circ} \mathrm{C}$ during ultrasonic treatment, the 


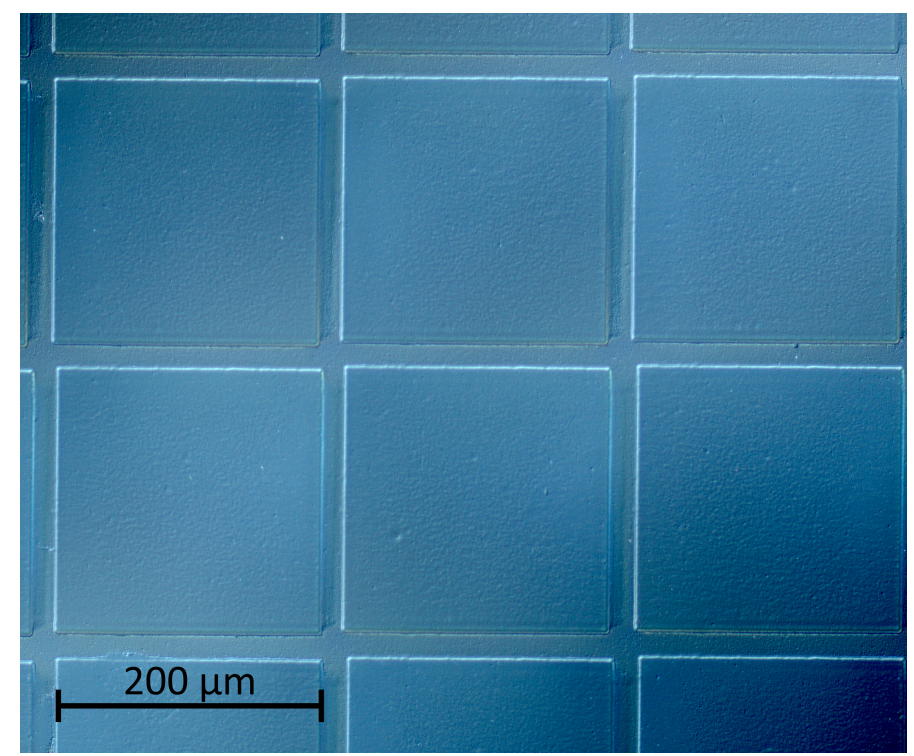

Figure 3.1: A reflected-light (RL) microscope image of the irradiated surface of a D263M cover glass taken with differential interference contrast (DIC). The $200 \mu \mathrm{m}$ sized quadratic spot was scanned with a period of $220 \mu \mathrm{m}$. Each position was irradiated with one laser pulse at a fluence of $2.0(1) \mathrm{J} / \mathrm{cm}^{2}$.

profiles retrieve their pristine shape.

For a quantitative analysis, I calculated the profiles of curvature difference $k^{(i)}(x)$, plotted in Figs. $3.2 \mathrm{c}$ and $3.2 \mathrm{~d}$, with respect to the curvature profiles of the pristine sample and $i=1,2$ marking the measurement direction. The diverging curvature at the ends of these profiles is an artifact of the smoothing procedure. Aside from this artifact, the curvature change is rather homogeneous. Therefore, it can be represented by the arithmetic mean $\left\langle k^{(i)}>\right.$ of all values of curvature change of the profile in between 2.5 and $17.5 \mathrm{~mm}$. I again averaged the values of $\left\langle k^{(i)}>\right.$ for both measurement directions $i=1,2$ to achieve the isotropic curvature change $\langle k\rangle$. For the data shown in Fig. 3.2 , it is $\langle k\rangle=-0.259(1) \mathrm{m}^{-1}$ after irradiation and $<k>=-0.0003(4) \mathrm{m}^{-1}$ after etching.

To investigate the effect of the irradiation pattern on the deformation, I irradiated two samples with the macroscopic patterns sketched in Fig. 3.3. The blue colored area was irradiated by stitching a homogenized laser spot (setup like in Fig. 2.2 with an approximate size of $1.1 \mathrm{~mm}^{2}$ with a bit of overlap. In both cases, one pulse per irradiation position was applied.

Fig. 3.4 shows the measured height profiles and calculated profiles of curvature difference for the sample irradiated as in Fig. $3.3 \mathrm{a}$ with a fluence of about $220 \mathrm{~mJ} / \mathrm{cm}^{2}$ in the homogeneous area of the laser spot. In the direction orthogonal to the transition line, the irradiated and non-irradiated areas can be clearly distinguished by the deformation (Fig. 3.4b and Fig. 3.4d), whereas in direction parallel to the transition line, a homogeneous curvature change can be observed (Figs. 3.4a and 3.4c). I also simulated the deformation of the sample by the FEM, assuming a $t_{s}=209 \mu \mathrm{m}$ thick substrate that is partly covered by a stressed film, of which I arbitrarily chose the film thickness to be $t_{f}=300 \mathrm{~nm}$. By use of the average curvature difference $k_{p}$ in 


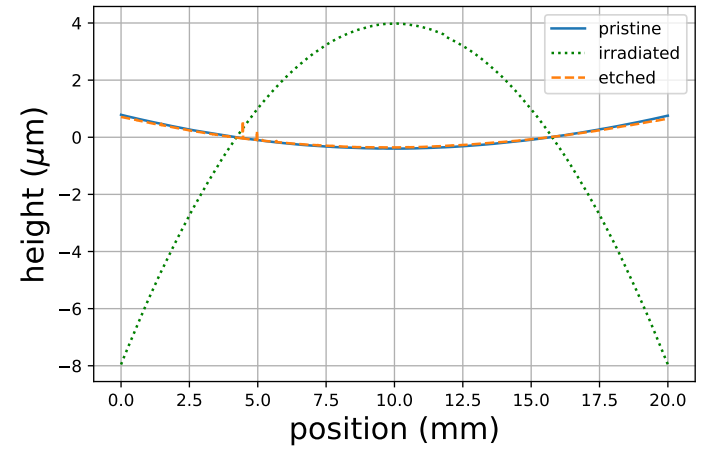

(a) Direction 1

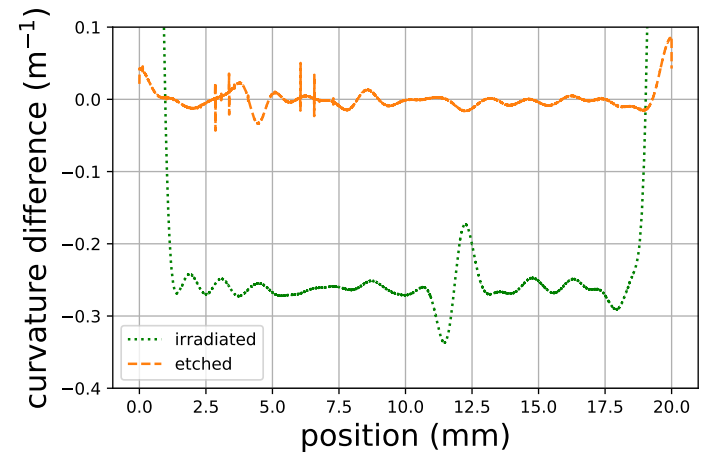

(c) Direction 1

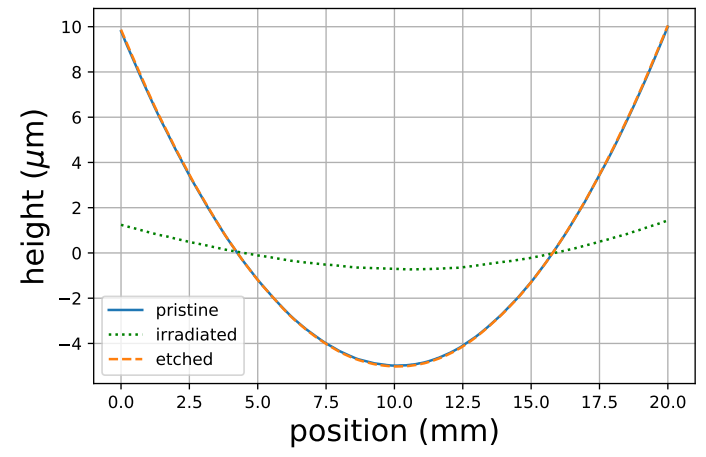

(b) Direction 2

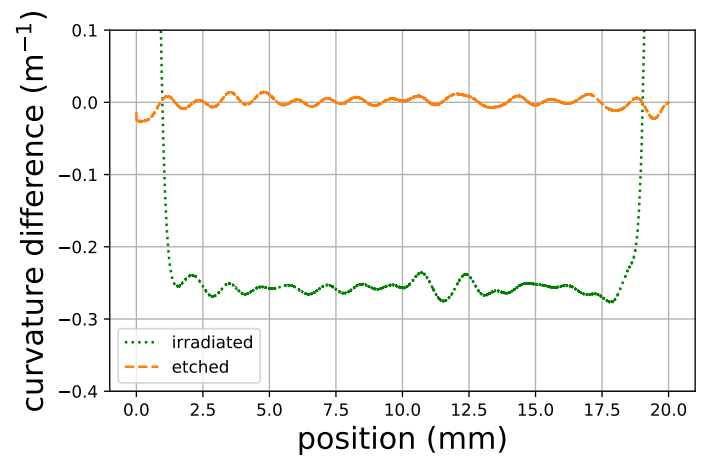

(d) Direction 2

Figure 3.2: An Example for deformation of a D263M cover glass by laser irradiation. One side of the sample was irradiated with a regular pattern of $200 \mu \mathrm{m}$ sized quadratic spots with a period of $220 \mu \mathrm{m}$ at one pulse per position and a fluence of $360(30) \mathrm{mJ} / \mathrm{cm}^{2}$. Subsequently, the sample was etched in $\mathrm{KOH}$ solution. The height profiles of the non irradiated side of the pristine sample, of the sample after irradiation and after etching are shown in (a) and (b) for two orthogonal measurement directions. Please note that there is an almost perfect overlap of profiles of the pristine sample and after etching. The corresponding profiles of curvature difference are plotted in $(\mathrm{c})$ and $(\mathrm{d})$.

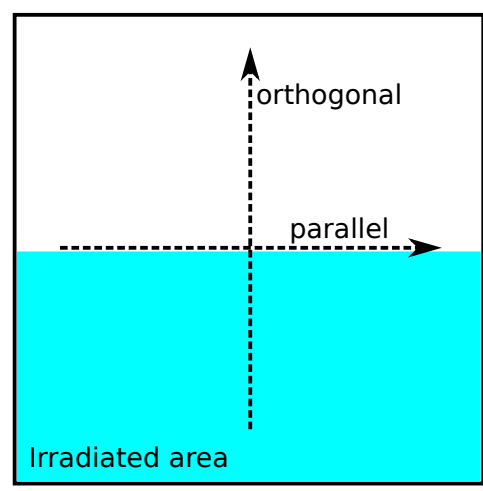

(a) half irradiated

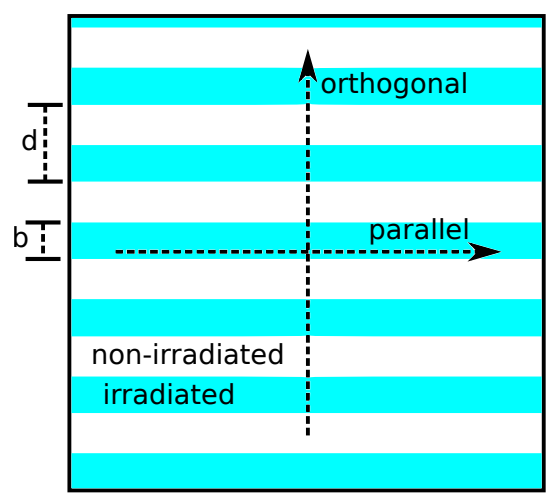

(b) irradiated with line pattern

Figure 3.3: Irradiation schemes for the samples shown in Figs. 3.4 and 3.5, respectively. The height profiles have been measured on the non-irradiated side of the sample and are indicated by the arrowed lines. In (b), the period was $d=4 \mathrm{~mm}$ and the line width $b=2 \mathrm{~mm}$. 
direction parallel to the transition line and the Stoney Eq. (1.1), I calculated the film stress $\sigma_{f}$ to be

$$
\sigma_{f}=-2 \frac{E_{s}}{6\left(1-\nu_{s}\right)} \frac{t_{s}^{2}}{t_{f}} k_{p}=252 \mathrm{MPa}
$$

with the Young's modulus $E_{s}$ and Poisson's ratio $\nu_{s}$ of Schott D263, as given in table A.1 in the appendix. Please note that a factor of -2 has been introduced to account for the fact that the surface is only half coated by the film and that the curvature of the non-irradiated sample side is considered. The simulated profiles agree very well with the measured data.

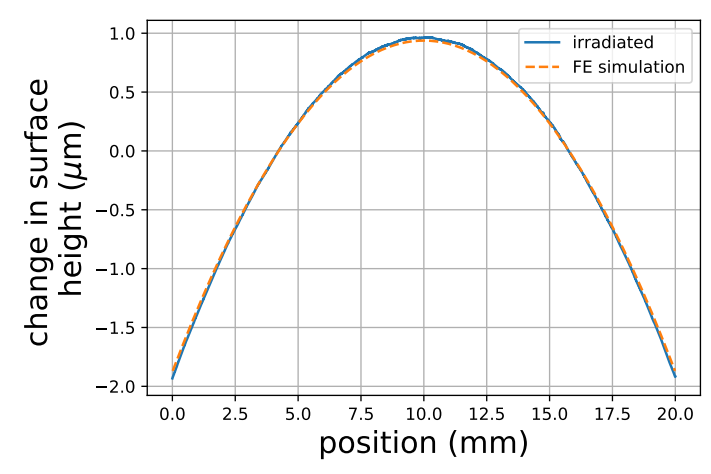

(a) Parallel to transition line

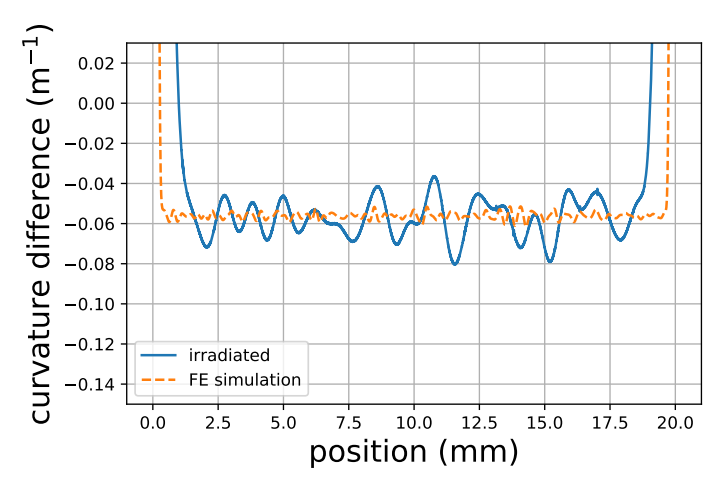

(c) Parallel to transition line

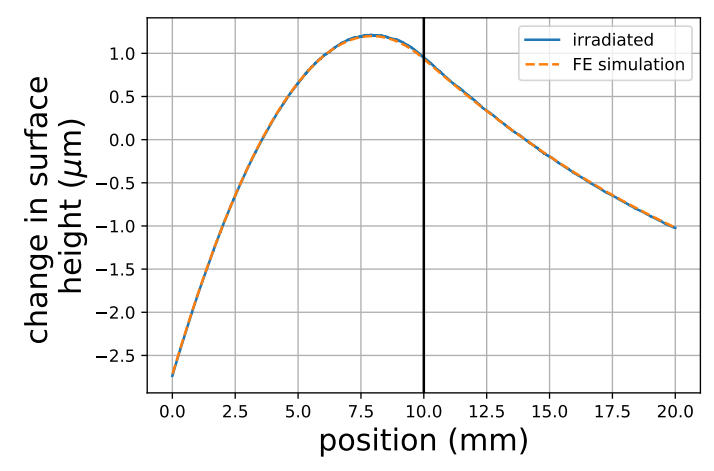

(b) Orthogonal to transition line, left side irradiated

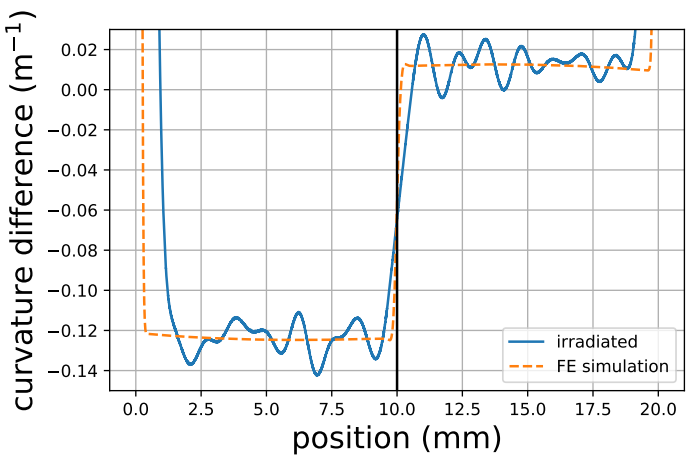

(d) Orthogonal to transition line, left side irradiated

Figure 3.4: Comparison of deformation of a D263M cover glass due to irradiation and the simulated deformation by the FEM. One half of one side of the sample was irradiated by a pattern of homogenized laser spots with a lateral size of about $1 \mathrm{~mm}$. The spot was scanned with a bit of overlap, a fluence of about $220 \mathrm{~mJ} / \mathrm{cm}^{2}$ and one pulse per position. For the finite element simulation, the experimentally determined integrated stress was applied. The change in surface height by irradiation is shown in (a) and (b) in directions parallel and orthogonal to the transition between irradiated and non irradiated area. In (c) and (d), the corresponding profiles of curvature difference are shown.

In Fig. 3.5 the experimental results are shown for the irradiation pattern sketched in Fig. $3.3 \mathrm{~b}$ for a fluence of about $2.0 \mathrm{~J} / \mathrm{cm}^{2}$. The pattern of wide lines is clearly 
visible in the profiles orthogonal to the lines (Fig. 3.5b and Fig. 3.5d). Again, parallel to the lines a homogeneous curvature change (Fig. 3.5c) is observed. For the simulation, a film stress of $\sigma_{f}=872 \mathrm{MPa}$ for a hypothetical film thickness of $t_{f}=300 \mathrm{~nm}$ was calculated. The calculated film stress is remarkably higher than in the preceding paragraph because of the higher laser fluence applied (cf. Fig. 3.6). Experiment and simulation fit very well. This example and the one in Fig. 3.4 demonstrate that the deformation of the samples can be understood by a stressed surface layer in the irradiated areas. This opens up a way to deform samples in a certain way, but also makes it necessary to gain a better understanding and characterization of the mechanism behind the generated stress, because deformation is also expected to occur at any instance such samples are irradiated by an ArF excimer laser, for example in the case of micro-structuring of the surface.

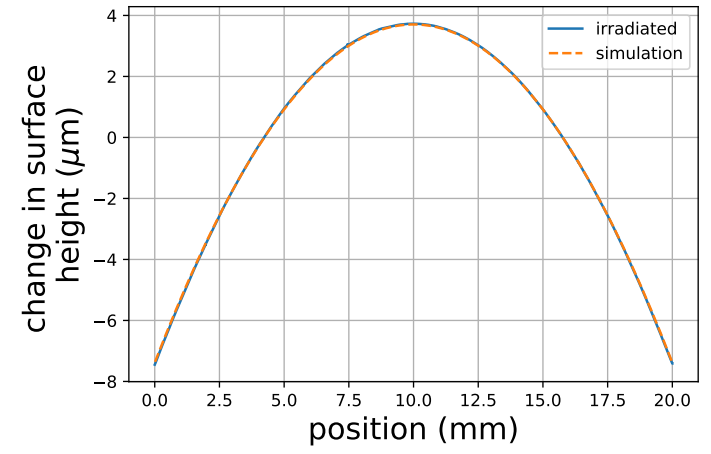

(a) Parallel to lines.

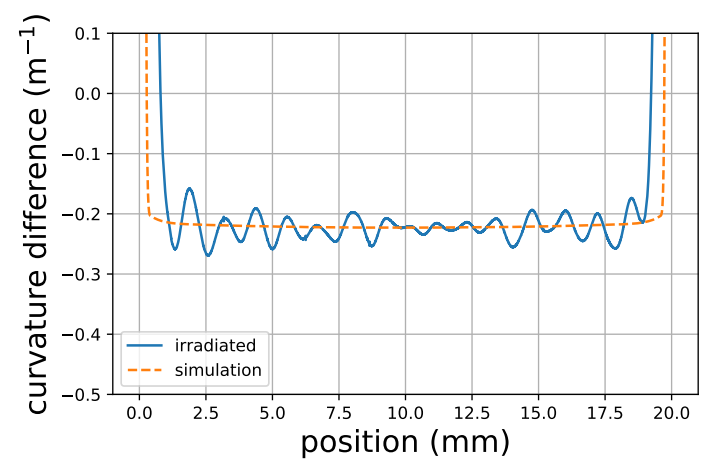

(c) Parallel to lines.

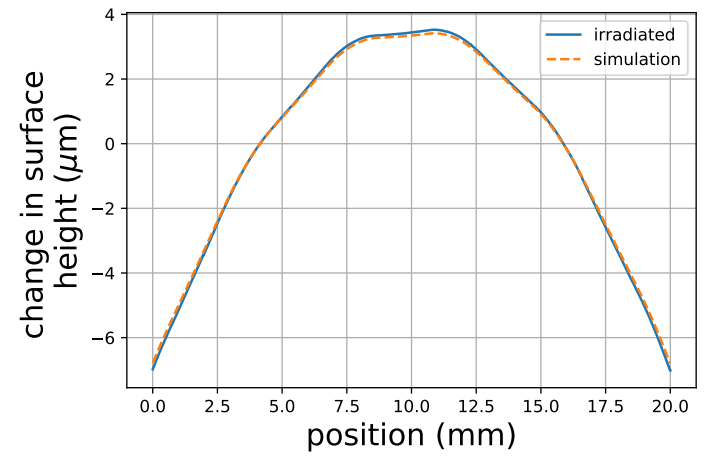

(b) Orthogonal to lines.

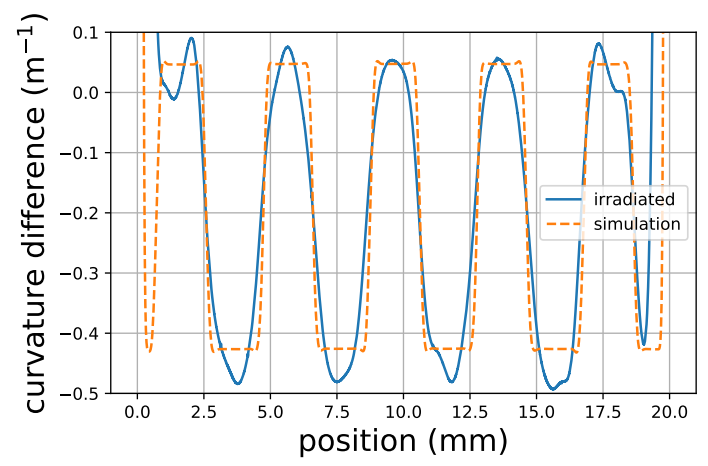

(d) Orthogonal to lines.

Figure 3.5: Comparison of deformation of a D263M cover glass due to irradiation and the simulated deformation by the finite element method. One side of the sample was irradiated by a line pattern of homogenized laser spots with a lateral size of about $1 \mathrm{~mm}$. The period of the line pattern is about $4 \mathrm{~mm}$ and the width of the lines is about $2 \mathrm{~mm}$ (to rows of spots). The spot was scanned with a bit of overlap, a fluence of $2.0 \mathrm{~J} / \mathrm{cm}^{2}$ and one pulse per position. For the FE simulation, the experimentally determined integrated surface stress was applied. The change in surface height by irradiation is shown in (a) and (b) in directions parallel and orthogonal to the lines. In $(c)$ and $(d)$, the corresponding profiles of curvature difference are plotted. 


\subsubsection{Integrated stress in dependence on fluence, pulse num- ber and spot area}

In the preceding section, I demonstrated that a surface-near and equibiaxial plane stress is generated by irradiation. For a quantitative analysis of the underlying mechanism for stress generation, I systematically varied the fluence, pulse number and spot size and calculated the integrated stress of the stressed surface layer by a slightly modified form of the Stoney Eq. (1.1).

Again, I irradiated one side of the sample by a pattern of non overlapping square spots and measured the average curvature difference $k$ to the pristine surface of the non irradiated side. I used this value for calculation of the integrated stress $N$ via a modified form of the Stoney Eq. (1.1)

$$
N \equiv \sigma_{f} \cdot t_{f}=-\frac{A_{\mathrm{tot}}}{A_{\mathrm{irr}}} \frac{E_{s} t_{s}^{2}}{6\left(1-\nu_{s}\right)} k .
$$

Here, $\sigma_{f}$ is the mean stress of a thin surface layer with thickness $t_{f}$. I introduced the factor $A_{\text {tot }} / A_{\text {irr }}$ of total area to irradiated area to account for the non irradiated space between neighboring irradiation positions and justified its introduction by a FE analysis of the deformation of a cover glass by a stressed thin film that is structured the same way as in the experimental case. I also introduced a factor -1 to account for the fact that I measured the curvature on the non-irradiated side.

Fig. 3.6 shows the integrated stress as a function of the laser fluence for one pulse per position, whereas a positive sign corresponds to a tensile stress inside the irradiated surface. The edge length of the laser spot was $200 \mu \mathrm{m}$ and the period of the irradiation pattern was $220 \mu \mathrm{m}$. A step-like dependence of the integrated stress is observed between 100 and $500 \mathrm{~mJ} / \mathrm{cm}^{2}$. For lower fluences the integrated stress is zero and for higher fluences it stays roughly at $250 \mathrm{~N} / \mathrm{m}$ up to a fluence of $2 \mathrm{~J} / \mathrm{cm}^{2}$. I etched four of the irradiated samples after irradiation in the same way as explained in the preceding section. Thereby, the curvature change and, accordingly, the integrated stress returned to zero. I also etched another six samples before irradiation. They follow the same trend as the samples without etching.

Inside the fluence range of the step in Fig. 3.6. I observed a transition in the height profiles of the irradiated surface. For fluences between 150 and $250 \mathrm{~mJ} / \mathrm{cm}^{2}$, the surface height increased inside the irradiated areas with respect to the nonirradiated areas in between. An example for this behavior is shown in Fig. 3.7a. In contrast, from $360 \mathrm{~mJ} / \mathrm{cm}^{2}$ upwards, the surface height decreased due to irradiation as shown in Fig. 3.7b, indicating the onset of ablation.

The dependence of the integrated stress on the number of pulses per position is plotted in Fig. 3.8a for three different fluences, namely 149(9), 250(10) and $1000(50) \mathrm{mJ} / \mathrm{cm}^{2}$. Again, I applied the regular pattern of $200 \mu \mathrm{m}$ sized spots and used a repetition rate of $\leq 10 \mathrm{~Hz}$ at each irradiation position. The data indicate no strong dependence on the number of pulses. However, an increase in the integrated stress from one to two pulses might be suspected.

By change of the projected mask in the optical setup (cf. Fig. 2.1), I achieved 


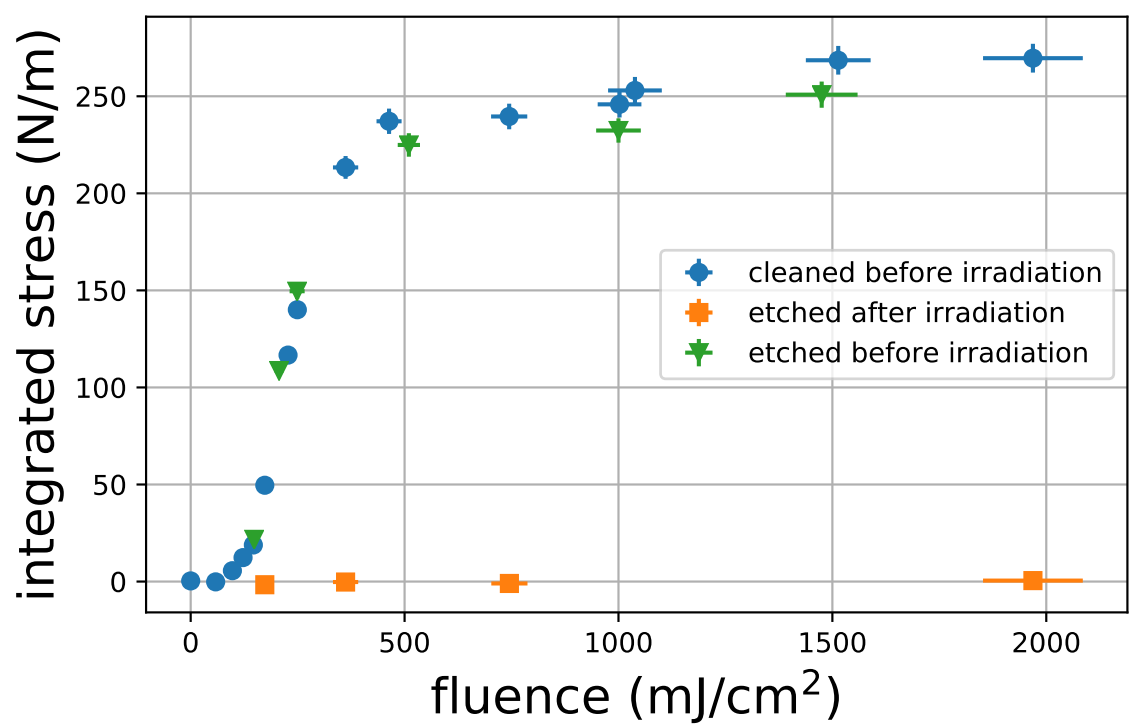

Figure 3.6: The measured integrated stress (positive sign: tensile stress) as a function of the laser fluence. Each sample was irradiated by a regular pattern of $200 \mu \mathrm{m}$ sized square spots with a period of $220 \mu \mathrm{m}$ at constant fluence and one pulse per position. Four of the samples were etched in $\mathrm{KOH}$ solution after irradiation. Another six samples were etched before irradiation.

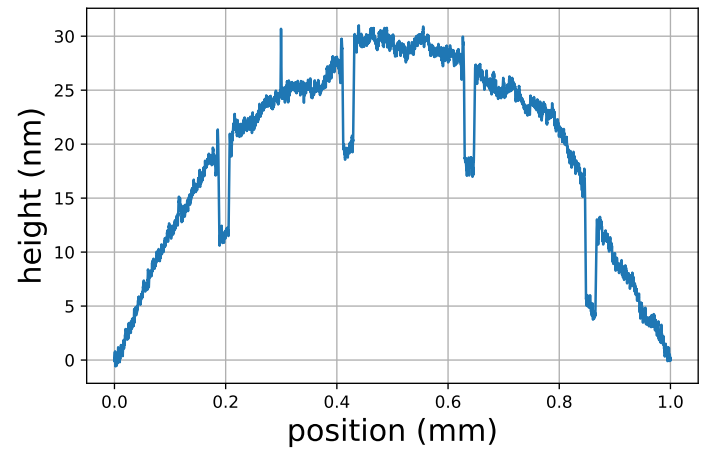

(a) $250(20) \mathrm{mJ} / \mathrm{cm}^{2}$

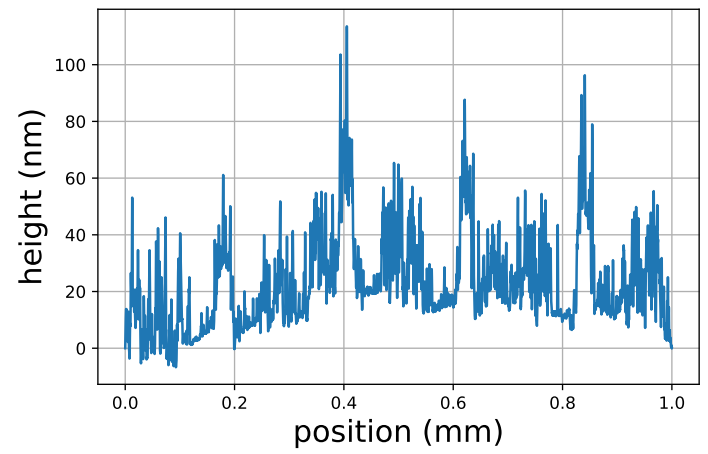

(b) $360(30) \mathrm{mJ} / \mathrm{cm}^{2}$

Figure 3.7: Height profiles measured by profilometry of the irradiated side (one pulse per position) of two of the samples shown in Fig. 3.6. At a fluence of $250(20) \mathrm{mJ} / \mathrm{cm}^{2}$ (a), the surface height increased by irradiation, while it decreased at a fluence of $360(30) \mathrm{mJ} / \mathrm{cm}^{2}$ (b). The high roughness in (b) is probably due to debris deposited after each laser pulse. 


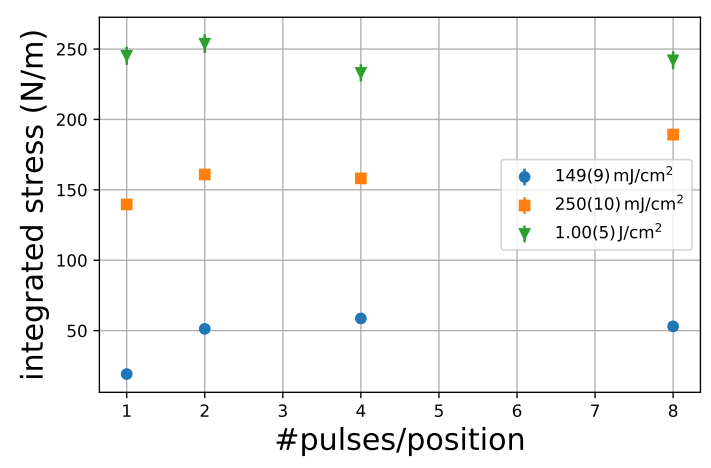

(a)

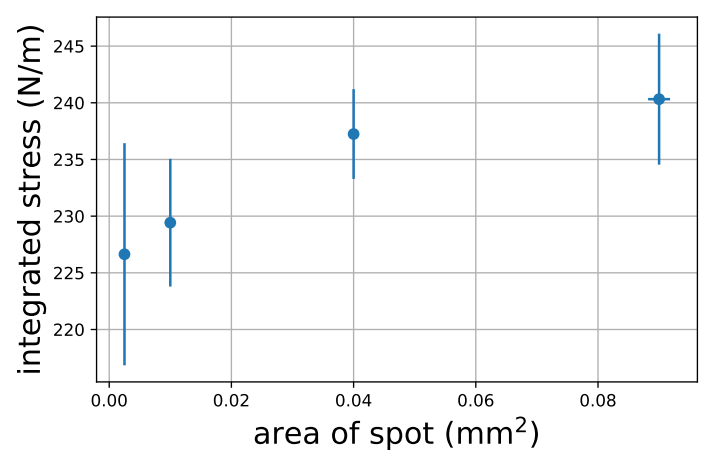

(b) $730(40) \mathrm{mJ} / \mathrm{cm}^{2}$

Figure 3.8: (a) Dependence of the integrated stress on the number of pulses per position for irradiation by a regular pattern of $200 \mu \mathrm{m}$ sized square spots with a period of $220 \mu \mathrm{m}$ at constant fluence. The repetition rate was chosen to be $\leq 10 \mathrm{~Hz}$ at each irradiation position. (b) Dependence on the spot size at one pulse per position and a fluence of $730(40) \mathrm{mJ} / \mathrm{cm}^{2}$. For a high coverage of the surface, the period of the irradiation pattern was varied.

different spot sizes for a fluence of $730(40) \mathrm{mJ} / \mathrm{cm}^{2}$ and one pulse per position. For a high coverage of the sample surface, the period of the irradiation pattern had to be adapted as well. The integrated stress in dependence on the area of the laser spot is plotted in Fig. 3.8b. It seems like there is a systematic increase in the integrated stress with increasing spot area. However, this is covered by the measurement uncertainty of the integrated stress. As the relative uncertainty in the spot size increases with decreasing spot size, the error of the integrated stress is largest for the smallest spot size applied. Thus, no significant dependence can be observed.

\subsubsection{Etching in potassium hydroxide solution}

In the preceding section, the maximum integrated stress that can be induced was determined to a value of about $250 \mathrm{~N} / \mathrm{m}$. Unfortunately, this value does not provide any information on the thickness of the stressed layer and on the stress distribution in direction normal to the surface. Both were obtained via etching experiments.

In the preceding sections, I already demonstrated that, by etching of irradiated samples in $\mathrm{KOH}$ solution, the pristine shape could be retained. For a quantitative analysis, I refined these experiments by abandoning the ultrasonification during etching, by use of a precisely mixed $1.000(2) \mathrm{mol} / \mathrm{l}(\approx 5.3 \mathrm{wt} \%$, supplier: Carl Roth) aqueous potassium hydroxide solution, by carrying out an improved temperature control, a monitoring of the change in structure height during etching and a measurement of the etch rate of the pristine material. The curvature difference plotted against the total etching time for a sample irradiated at $0.99(5) \mathrm{J} / \mathrm{cm}^{2}$ with a pattern of $200 \mu \mathrm{m}$ sized square spots with a pitch of $220 \mu \mathrm{m}$ and one pulse per position is plotted in Fig. 3.9a in dependence on the total etching time. The curvature difference increases until at about $45 \mathrm{~min}$ a value of zero curvature is reached. A control sample, which was simultaneously 'etched' in deionized water, exhibited only 
a slight dependence on the etching time (not shown). Based on the data shown in Fig. 3.9a, I estimated the stress distribution along the axis normal to the surface by the procedure described below.

Fig. $3.9 \mathrm{~b}$ shows the data for determination of the etch rate at $80^{\circ} \mathrm{C}$ of the pristine material. I measured the thickness of a non irradiated D263M cover glass after seven etching steps with a micrometer gauge. At each step, I calculated the average of ten measurement results and subsequently estimated the uncertainty of the average value by the standard deviation of the ten measured values. By a linear fit, I determined the thickness change to be $-920(70) \mathrm{nm} / \mathrm{h}$. As both sample sides were etched during the experiment, the etch rate is half of this value.

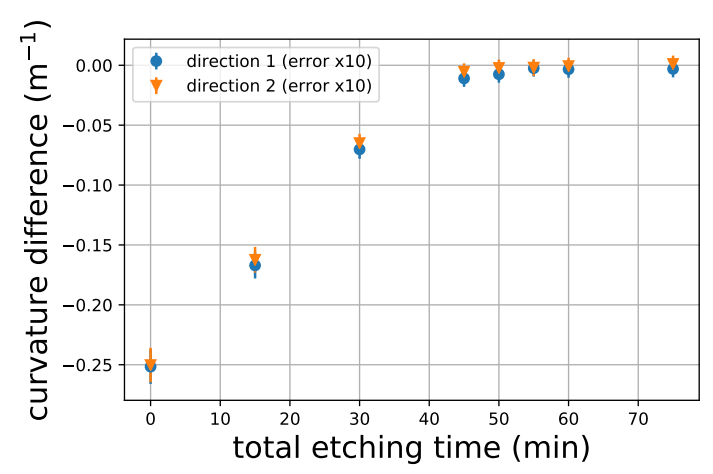

(a)

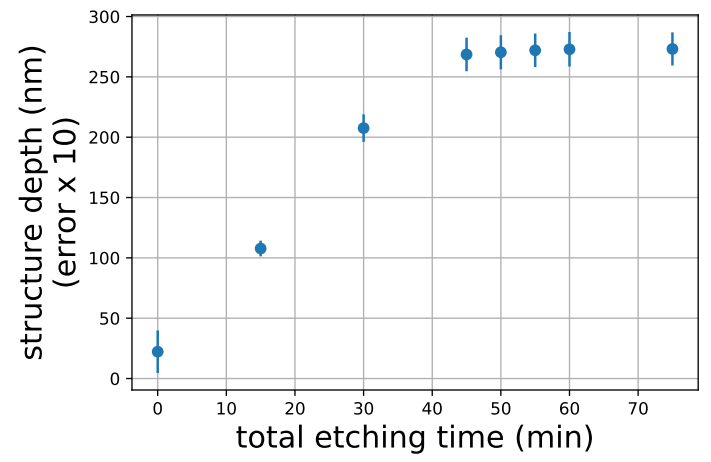

(c)

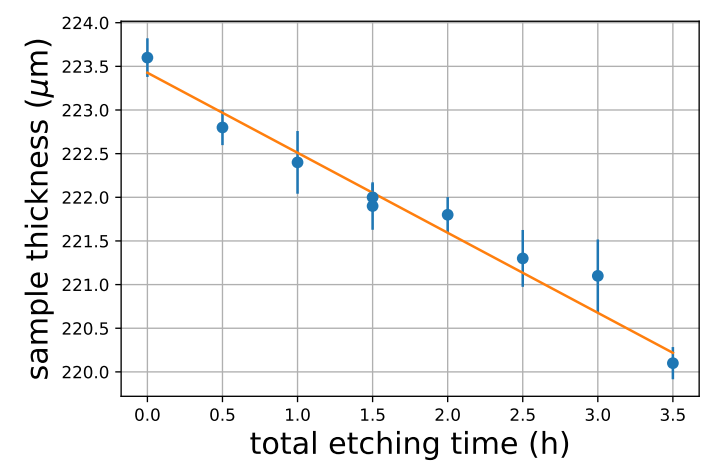

(b)

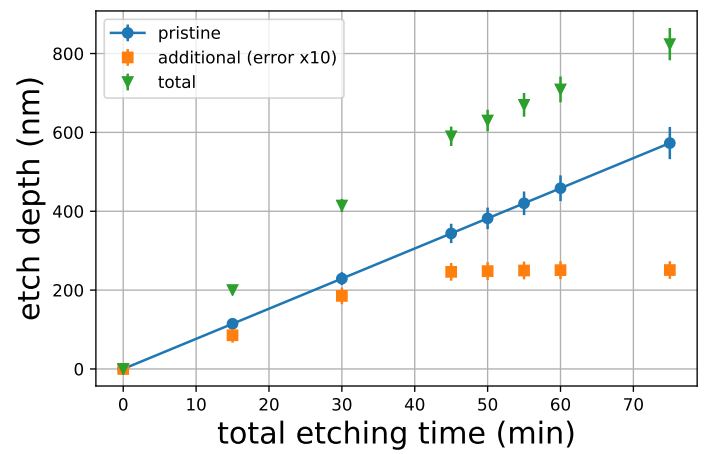

(d)

Figure 3.9: Demonstration of results for the refined etching procedure. (a) The curvature difference of a sample irradiated at $0.99(5) \mathrm{J} / \mathrm{cm}^{2}$ with one pulse per position plotted against the total etching time. (b) The thickness of a pristine sample etched the same way as in (a) as a function of total etching time. (c) The structure depth for the same irradiation conditions as in (a) as a function of total etching time. (d) By combination of the results of (b) (pristine) and (c) (additional), I calculated the total etch depth of the irradiated material (total).

For determination of the etch rate of the irradiated material, I irradiated a D263M cover glass with only a few spots, each of which was at least $2 \mathrm{~mm}$ apart from its neighbor, and measured the structure height of five identical spots before and between the etching steps. The large distance of the spots served for reduction of the influence of debris on the measurement results. Fig. $3.9 \mathrm{c}$ shows the average structure 
depth in dependence on the total etching time for six different measurements at 5 different spots. The structure depth increases almost linearly with increasing etching time until $45 \mathrm{~min}$. For longer times, it stays constant. This shows that the material modified by irradiation is etched faster than the pristine material. A control sample similarly 'etched' in water instead of potassium hydroxide solution exhibited a small increase of about $15 \mathrm{~nm}$ in structure height after the first etching step (not shown). For the subsequent steps, no relevant change in structure depth was observed.

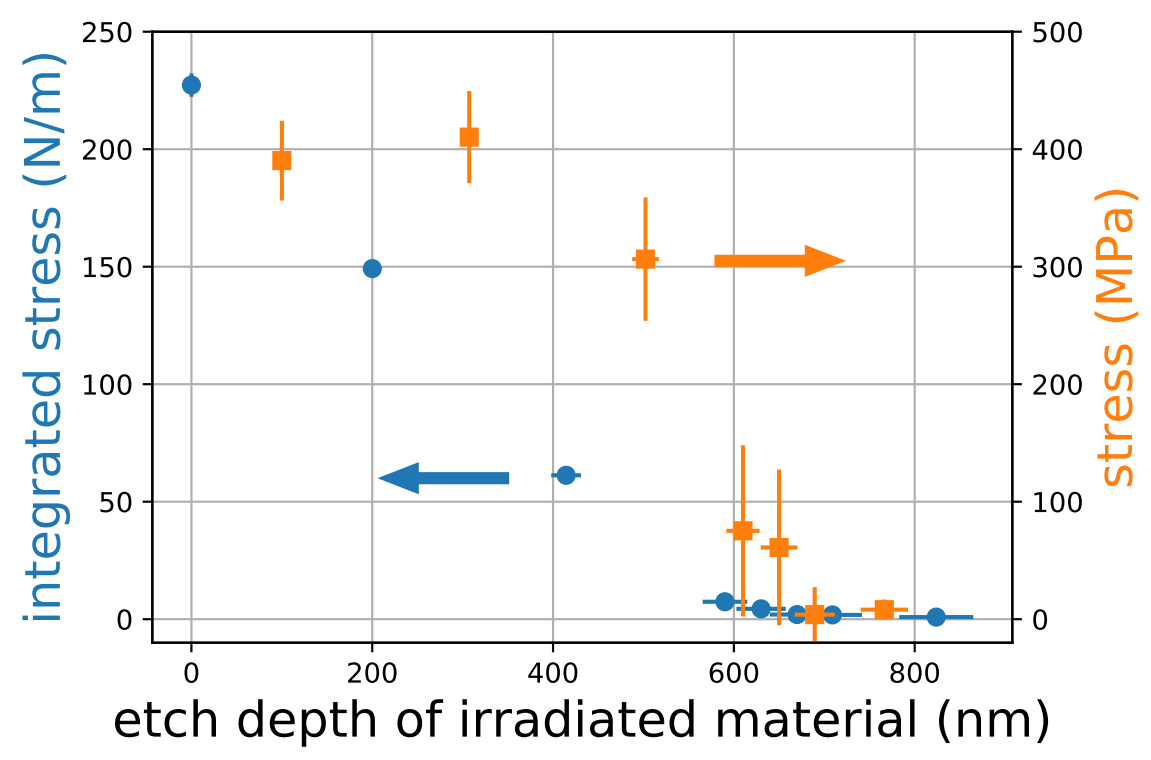

Figure 3.10: Results of the etching experiment on a sample irradiated with $0.99(5) \mathrm{J} / \mathrm{cm}^{2}$ and one pulse per position. The integrated stress is plotted against the total etching depth on the left ordinate (blue circles). The stress, estimated by differentiation of the integrated stress, is plotted on the right ordinate (orange squares).

By combination of the etch rate of the pristine material and the measured change in structure depth, I calculated the total etch depth for each time step. This is shown in Fig. 3.9d, where the calculated etch depth of the pristine material, the structure depth (additional) and the sum of both (total etch depth) are plotted against the total etching time. By use of the modified Stoney Eq. (3.2), I calculated the integrated stress at every time step and, by use of the data in Fig. 3.9d, I plotted it against the etch depth (Fig. 3.10). The integrated stress decreases almost linearly, which is an indication for a constant stress value, until a depth of about $600 \mathrm{~nm}$ is reached. By differentiation of this data via a central difference method, the stress at a certain etch depth can be roughly estimated. This is also demonstrated in Fig. 3.10. Please note that the points of the stress have been positioned in the middle position between the measurement points used for calculation. Fig. 3.10 shows that a stress of about $400 \mathrm{MPa}$ is reached inside the surface. This stress roughly stays constant up to a depth of $500 \mathrm{~nm}$ and decreases sharply for higher depths. In between a depth of 600 to $700 \mathrm{~nm}$, the stress has totally vanished. Thus, the stressed surface layer has a total thickness of about $650 \mathrm{~nm}$. For a laser fluence of $2.0(1) \mathrm{J} / \mathrm{cm}^{2}$, I similarly (with less precision) measured a thickness between 500 and $800 \mathrm{~nm}$ of the stressed surface layer. 
I need to mention that after etching of the irradiated samples, cracks were observed under the light microscope inside some of the ablation spots (Fig. 3.11). These cracks had not been observed before the etching procedure. Thus, either they were covered by debris or they grew or were created during etching. A systematic study, in which the pulse number was varied, showed that similar cracks can be observed at pulse numbers $>15$ at a fluence of $1 \mathrm{~J} / \mathrm{cm}^{2}$ for non-etched samples.

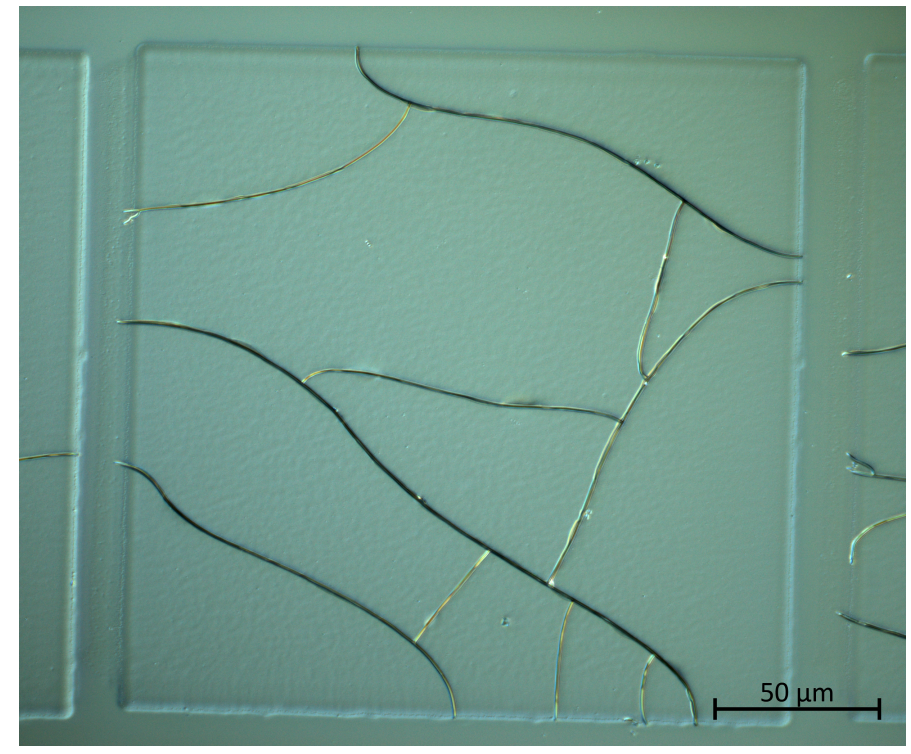

Figure 3.11: A DIC light microscope image of an ablation spot $\left(2.0(1) \mathrm{J} / \mathrm{cm}^{2}, 1\right.$ pulse) on the surface of a sample after etching. A few cracks can be observed, which are confined to the irradiated area. Before etching, no cracks could be observed. For most of the samples only some ablation spots exhibited cracks, and for most of these spots the number of cracks was less than in the present example.

\subsubsection{Antibiaxial plane stress component by line pattern}

So far, the overall curvature difference was the same in both measurement directions, which indicates that equibiaxial plane stress components were generated. It is known that antibiaxial stress components can be generated by structuring a stressed film attached to the underlying substrate [3, 19, 20]. For this, a line pattern of an aspect ratio $\gtrsim 0.1$ can be used (cf. Fig. 1.3b). To reproduce this result with laser generated surface stresses, I changed the quadratic mask in the irradiation setup (cf. Fig. 2.1) to a mask with a $5 \cdot 5 \mathrm{~mm}^{2}$ sized field of lines with a period of $80 \mu \mathrm{m}$. The resulting laser spot with an edge length of about $500 \mu \mathrm{m}$ was scanned in a regular pattern over the surface of one side of the sample with a distance between the irradiation positions of $520 \mu \mathrm{m}$. In between the samples, I varied the number of pulses and the fluence. In the following, I estimated the fluence in the lines of the projected line pattern by assuming a binary fluence distribution with a duty cycle (irradiated area divided by total area) of 0.5 inside the laser spot. This approach neglects any loss of contrast of the optical image compared to the mask.

The surface of a sample irradiated with 20 pulses per position and a nominal 
fluence of $0.9 \mathrm{~J} / \mathrm{cm}^{2}$ is shown in Fig. 3.12. The line pattern has been successfully transferred to the sample surface, as can be seen under the light microscope (Fig. 3.12a). However, it is more pronounced in the center of the spot than at the border, probably due to spherical aberrations of the projection lens. For taking the scanning electron microscopy (SEM) image shown in Fig. 3.12b, I cleaned the sample in a $\mathrm{KOH}$ based detergent (Borer Chemie DECONEX $15 \mathrm{PF}$ ) in an ultrasonic bath to remove the debris deposited during ablation and deposited a thin $(\approx 20 \mathrm{~nm})$ gold film on the surface. Pronounced grooves can be observed.
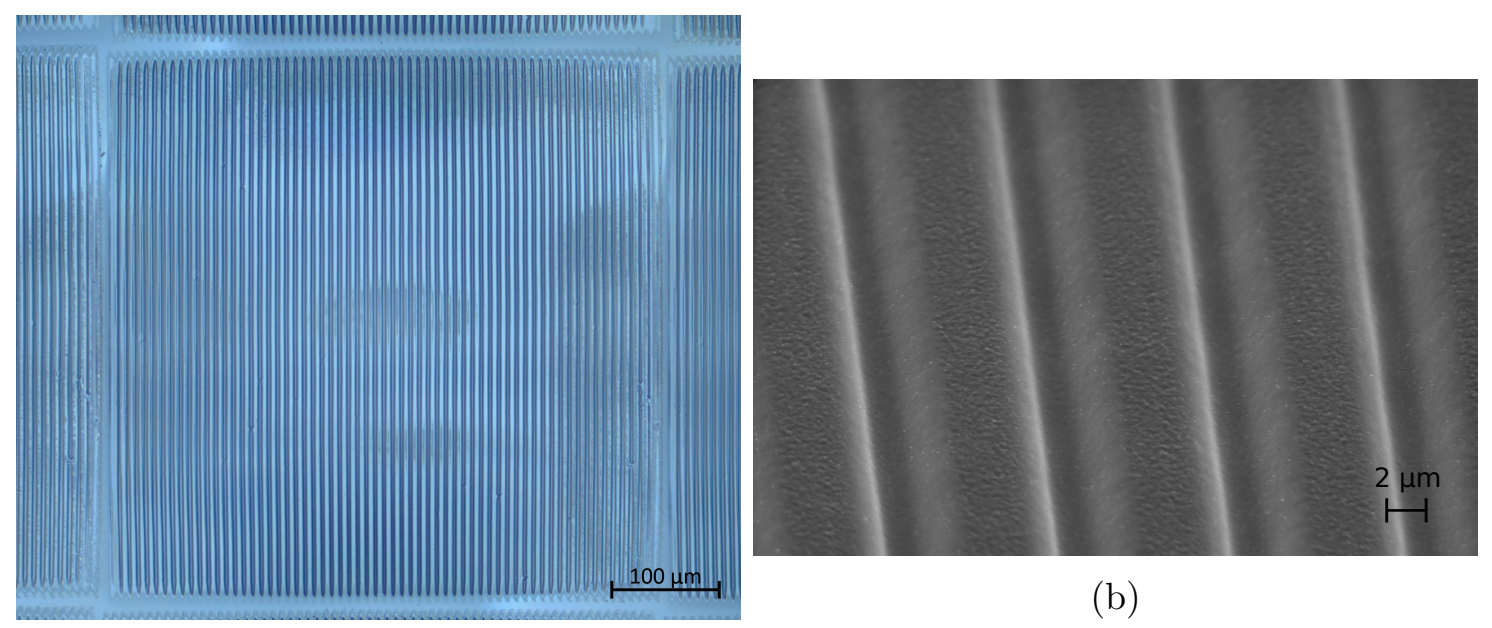

(b)

(a)

Figure 3.12: An example for a sample irradiated by a line pattern. (a) A RLDIC microscope image of the irradiated surface. The about $500 \mu \mathrm{m}$ sized spot was scanned over the sample surface with a pitch of $520 \mu \mathrm{m}$. The spot exhibits a line substructure with a period of about $8 \mu \mathrm{m}$. At each position, the fluence (assuming a duty cycle of 0.5 ) was $0.9 \mathrm{~J} / \mathrm{cm}^{2}$ and the pulse number was 20. (b) A SEM image of the lines in the center of the spot after cleaning in a $\mathrm{KOH}$ based solution and deposition of a thin gold film. The sample was inclined by $45^{\circ}$.

The curvature difference of the sample shown in Fig. 3.12 after irradiation is plotted in Fig. 3.13 for the directions along and across the lines. In both cases, the curvature difference is uniform, but the magnitude differs by a factor 0.6. Thus, an antibiaxial stress component has been generated by irradiation with the line pattern.

As a measure of anisotropy, I calculated the curvature ratio $k_{s} / k_{p}$ of average curvature difference in directions across $\left(k_{s}\right)$ and along $\left(k_{p}\right)$ the lines, respectively. This is plotted against the pulse number for different fluences in Fig. 3.14a. It decreases with increasing pulse number. To check if the ridges of the line structure have also been irradiated, I neglected the line substructure of the laser spot and calculated the integrated stress $N$ corresponding to the average curvature difference $k=\left(k_{s}+k_{p}\right) / 2$ via the modified Stoney Eq. (3.2). The obtained values of the integrated stress are plotted in Fig. $3.14 \mathrm{~b}$ for different pulse numbers against the calculated fluence (assuming a duty cycle of 0.5) in the trenches. The integrated stress saturates at a value of about $240 \mathrm{~N} \mathrm{~m}^{-1}$ for a large number of pulses. Because this value is close to the maximum value for irradiation with a uniform fluence 


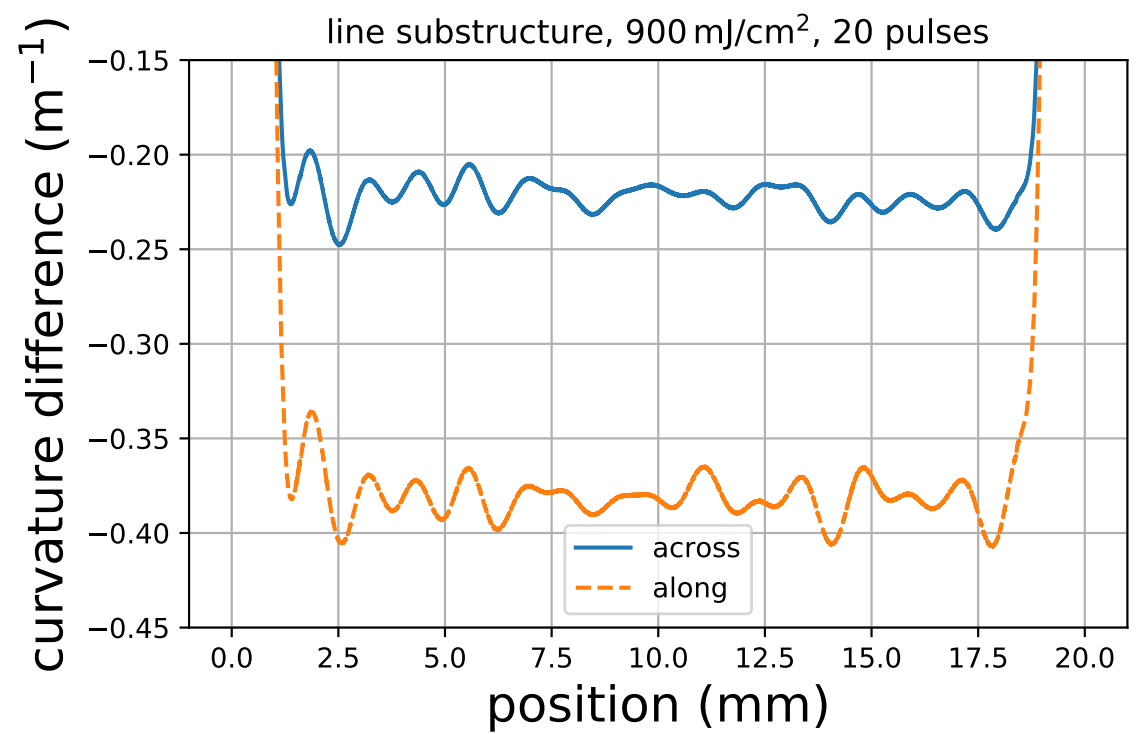

Figure 3.13: The profiles of curvature difference in directions across and along the lines of the sample shown in Fig. 3.12 after irradiation.

distribution (cf. Fig. 3.6) of $250 \mathrm{~N} / \mathrm{m}$, it seems plausible that by irradiation a stress was not only generated in the trenches but also on the ridges of the line pattern.

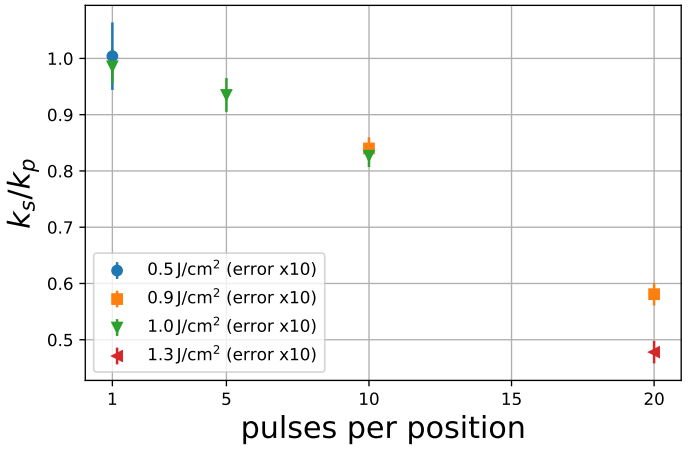

(a)

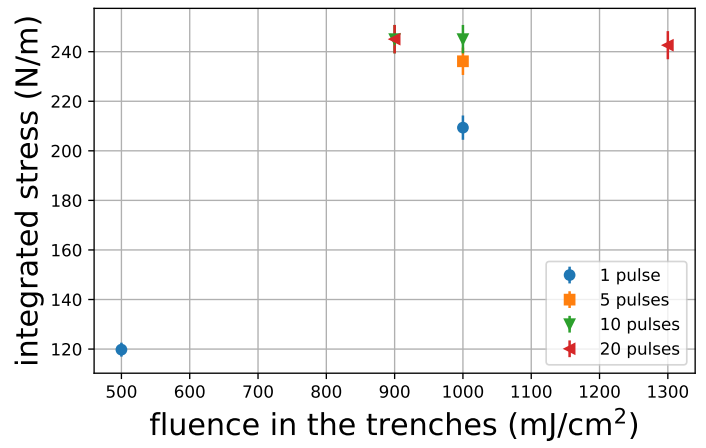

(b)

Figure 3.14: (a) Plot of the curvature ratio $k_{s} / k_{p}$ of curvatures across and along the lines against the pulse number per position for different fluences. The error of the curvature ratio has been magnified by a factor of ten for better visibility. (b) The calculated integrated stress as a function of calculated fluence in the trenches of the lines for the same samples as in (a).

For estimation of the structure depth, I obtained height profiles of the irradiated surfaces via tactile profilometry. However, because of the finite size of the stylus (cf. Eq. (2.1) for the maximum measurable amplitude of a sinusoidal profile), the results were unreliable for large structure depths. Therefore, for the two samples of largest structure depth, atomic force microscopy (AFM) measurements were performed by Jens Oltmanns (IFNano). Unfortunately, the measurements took place after cleaning of the samples for SEM analysis. Thus, structure depth could have already 
slightly changed compared to the irradiation step. Nevertheless, the curvature ratio $k_{s} / k_{p}$ (directly after irradiation) is plotted in Fig. 3.15in dependence on the structure depth. It decreases with increasing depth. In Fig. 3.15 also the curvature ratio as predicted by the Theory of Wikström et al. for a duty cycle of 0.5, Eq. (1.4), is plotted. In Sec. 3.2.3, a simple combination of this theory with the one of Stoney is made, that is also plotted.

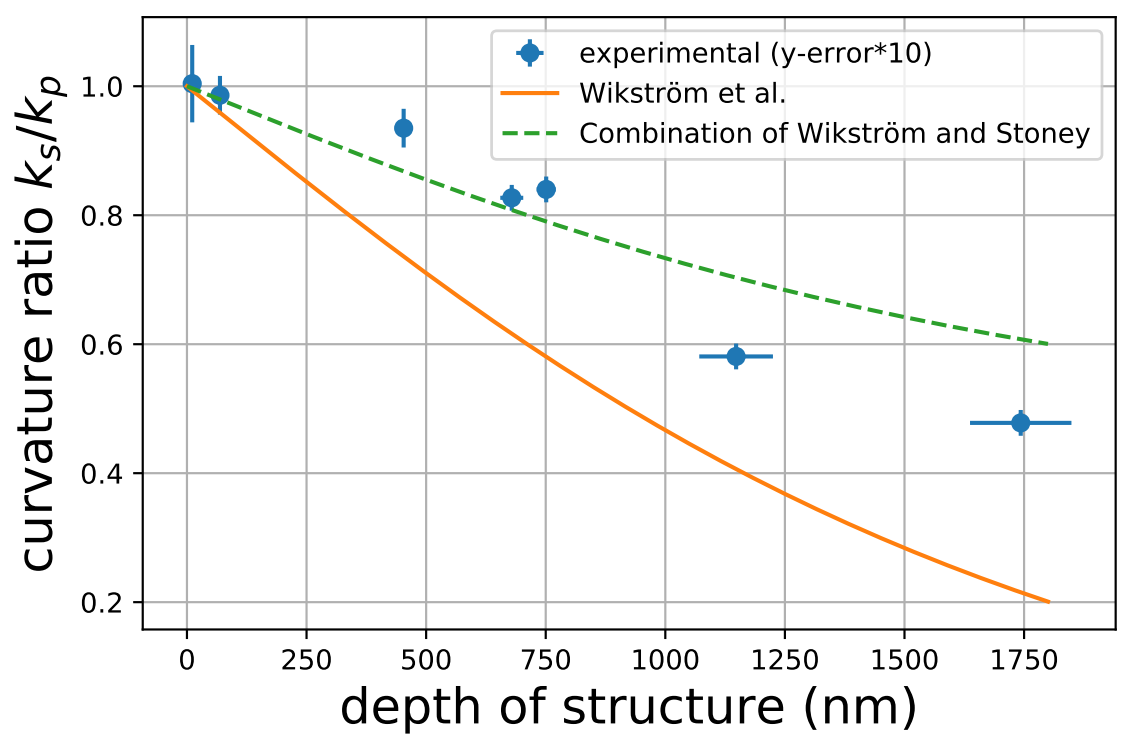

Figure 3.15: The curvature ratio $k_{s} / k_{p}$ of curvature across and along the direction of lines with a period of $8 \mu \mathrm{m}$ as a function of the depth of the lines. I calculated the theoretical dependencies by Eq. (1.4) (Wikström) and Eq. (3.11) (Wikström and Stoney) derived in Sec. 3.2.3. The error of the curvature ratio has been magnified by a factor of ten for better visibility.

For SEM analysis, I cleaned some of the samples in a $\mathrm{KOH}$ based detergent. As demonstrated in the preceding sections, this leads at least to a partial removal of the stressed surface layer. Surprisingly, also the ratio $k_{s} / k_{p}$ of curvature change across and along the lines was reduced by this procedure, as can be seen in Fig. $3.16 \mathrm{~d}$. For the sample irradiated at the highest fluence of $1.3 \mathrm{~J} / \mathrm{cm}^{2}$ and the highest pulse number of 20 pulses per position, cracks were observed after the cleaning step. Under the microscope (Fig. 3.16a) cracks can be seen at the border of the spot. Additionally, inside each trench of the line structure runs a crack, as can be observed via SEM analysis (Fig. 3.16b). 


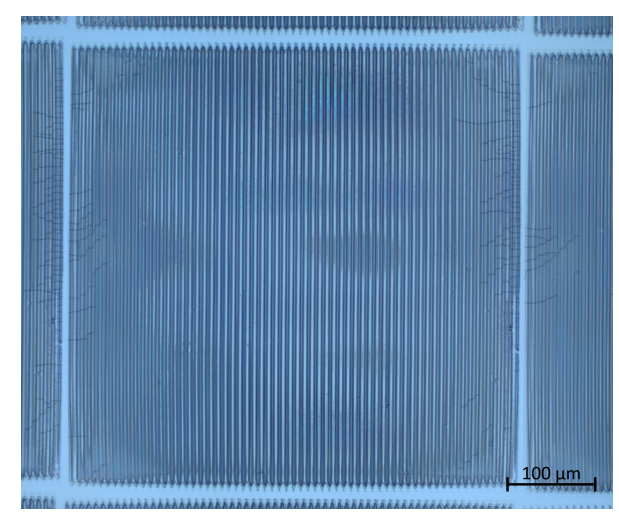

(a)

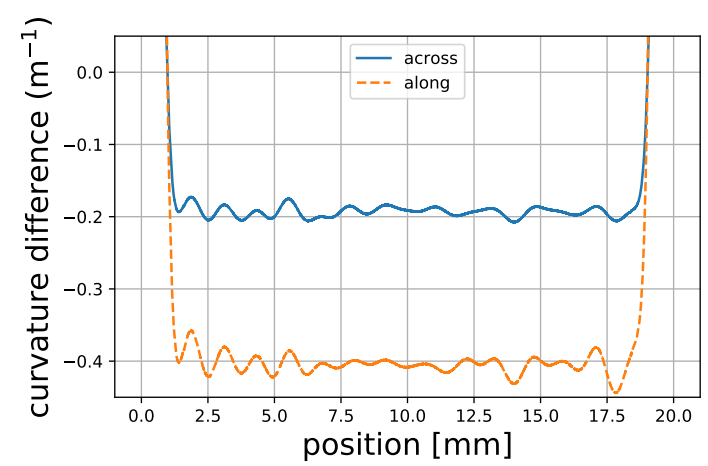

(c) After irradiation.

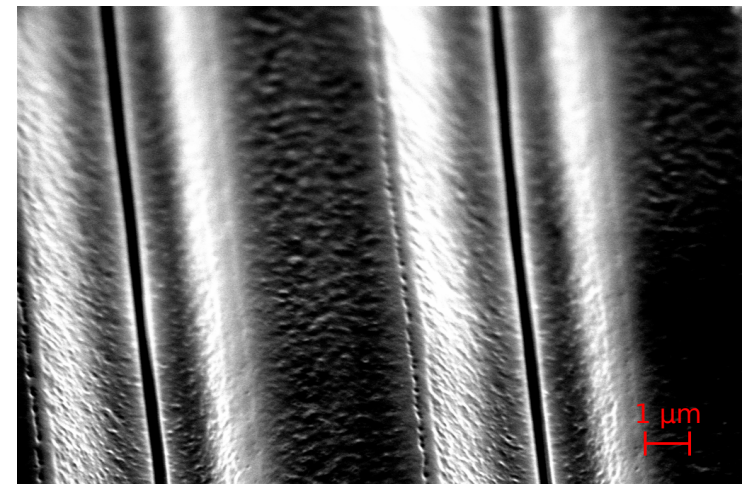

(b)

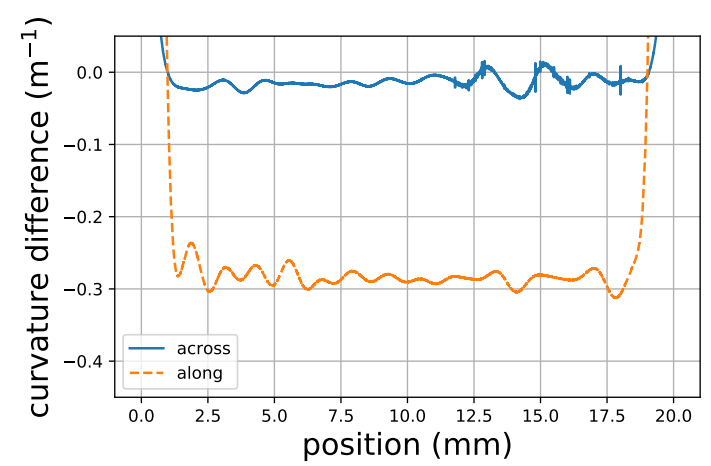

(d) After cleaning in $\mathrm{KOH}$ based solution.

Figure 3.16: The sample irradiated with a fluence of $1.3 \mathrm{~J} / \mathrm{cm}^{2}$ and 20 pulses per position. (a) A RL-DIC light microscope image after cleaning in $\mathrm{KOH}$ based solution. Please note the cracks at the boundary of the spot. (b) An SEM image of the line pattern obtained at $45^{\circ}$ inclination after cleaning. A crack runs at the bottom of the trench. (c) The profiles of curvature difference after irradiation. (d) The profiles of curvature change after the cleaning step. The curvature ratio changed from $0.478(2)$ to $0.048(2)$.

\subsubsection{Long-term temporal evolution of the integrated stress}

For an application of the above discussed deformation by excimer irradiation, stability of the generated surface stress is desirable. Hence, I measured the curvature difference with respect to the pristine curvature of samples irradiated with $200 \mu \mathrm{m}$ sized spots and 1 pulse per position in dependence on storage time at room temperature in ambient air. The results are shown in Fig. $3.17 \mathrm{a}$ for a fluence of $250 \mathrm{~mJ} / \mathrm{cm}^{2}$ and a fluence of $1 \mathrm{~J} / \mathrm{cm}^{2}$. There is a significant decrease of the curvature, which slows down over time. The relative curvature change is less for the sample irradiated at $1 \mathrm{~J} / \mathrm{cm}^{2}$ than for the sample irradiated at $250 \mathrm{~J} / \mathrm{cm}^{2}$. This also holds for the absolute values of curvature difference (not shown). Thus, the generated surface stress is not stable over time.

Fig. 3.17b shows the normalized curvature difference in dependence on the total annealing time in air for irradiation at a fluence of $1 \mathrm{~J} / \mathrm{cm}^{2}$. One sample was annealed at $100^{\circ} \mathrm{C}$, while the other sample was simultaneously stored outside the oven at 


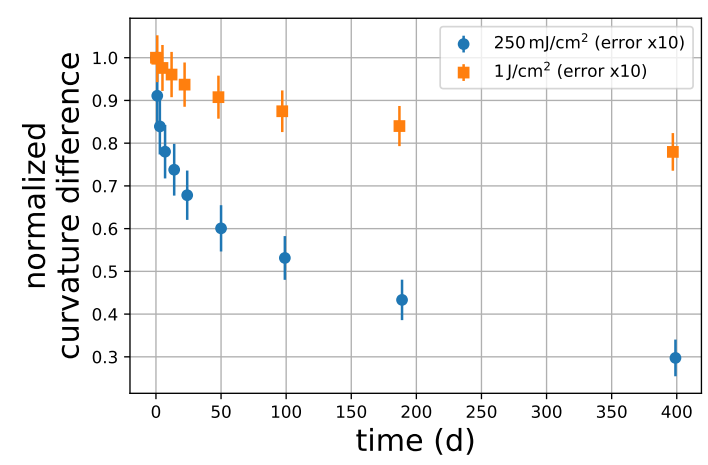

(a) room temperature, ambient air

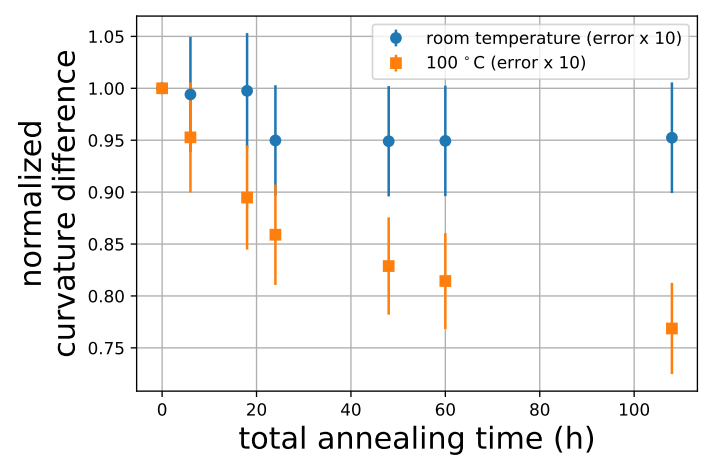

(b) $1 \mathrm{~J} / \mathrm{cm}^{2}$, ambient air

Figure 3.17: (a) The curvature difference normalized to the value after irradiation in dependence on the storage time in days for two samples irradiated at different fluences. The samples were stored at room temperature in ambient air. (b) The normalized curvature difference plotted against the total annealing time for annealing at $100^{\circ} \mathrm{C}$ in ambient air and for a fluence of $1 \mathrm{~J} / \mathrm{cm}^{2}$. The results of a control sample for 'annealing' at room temperature are also shown. In both figures, irradiation was done by a pattern of $200 \mu \mathrm{m}$ sized squares with a period of $220 \mu \mathrm{m}$ and one pulse per position. The errorbars for the relative curvature difference have been magnified by a factor of 10 for better visibility.

room temperature. The normalized curvature difference decreases clearly faster at $100{ }^{\circ} \mathrm{C}$ than at room temperature. Interpretation of the course of the normalized curvature difference in Fig. $3.17 \mathrm{~b}$ has to be done with care, because, as was shown in Fig. 3.17a, also the storage time of the samples between the annealing steps has an influence on the curvature. A non-irradiated sample, that was simultaneously annealed at $100^{\circ} \mathrm{C}$, did not exhibit a relevant change in curvature.

The samples were analyzed for cracks under the light microscope. Neither after storage for more than $170 \mathrm{~d}$ in ambient conditions nor after annealing for more than $100 \mathrm{~h}$ at $100^{\circ} \mathrm{C}$, cracks could be observed. I also observed a decrease in normalized curvature for a sample treated in deionized water at a temperature of $80^{\circ} \mathrm{C}$ (not shown). In this case, I found cracks inside some of the about $1 \cdot 10^{4}$ spots.

\subsubsection{Application for figure correction}

The described laser generated surface stresses could be applied for figure correction of thin mirrors. The approach for a proof of principle of this application is sketched in Fig. 3.18. A deformation of a cover glass was induced by an approximately $90 \mathrm{~nm}$ thick film of chromium, which was deposited by electron beam evaporation 1 . I chose chromium because of its good adhesion to silicate glass surfaces and high melting temperature, that allows for large tensile intrinsic stresses if deposited as a thin film [124, 125]. For example, for a $120 \mathrm{~nm}$ thick film of chromium on a D263M cover glass, I measured an average stress of about $1.4 \mathrm{GPa}$. After the deposition,

\footnotetext{
${ }^{1}$ The deposition was done by L. J. Richter (IFNano).
} 
I compensated the deformation induced by the chromium film by irradiating the non-coated side of the sample with a pattern of $200 \mu \mathrm{m}$ sized squares and one pulse per position. For this, according to Fig. 3.6, one could in principle choose a uniform irradiation pattern and adjust the fluence at every irradiation position to achieve the right local value of equibiaxial integrated stress. But, for simplicity, I used a different approach: From Fig. 3.6, I chose an appropriate fluence, that was kept constant during irradiation. In contrast, I adjusted the irradiation pattern to achieve a certain integrated stress at a certain position. This approach is similar to the one in [126] for lithographically structuring the oxide film on the backside of a silicon substrate in hexagons of different size.

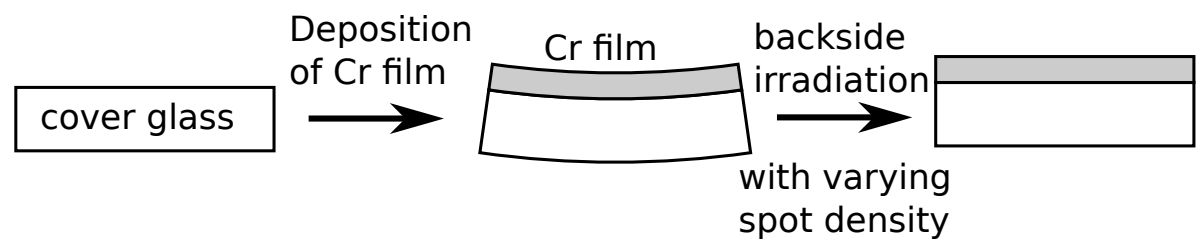

Figure 3.18: Scheme for a proof-of-principle for figure correction of thin substrates. A D263M cover glass is coated by a thin, continuous or non-continuous chromium film. Due to the tensile film stress, the cover glass is deformed. This deformation is compensated by irradiating the backside (non-coated side) of the sample surface with a proper irradiation pattern at a constant fluence.

Here, I show the results for one sample in detail. Before and after the chromium deposition, I measured a height map of the coated side (front side) of the sample by tactile profilometry. The difference of these height maps provides the deformation of the sample due to the coating, which is shown in Fig. 3.19a. A bowl shaped deformation can be observed in agreement with a tensile film stress. The RMS deformation and the peak-to-valley (PV) deformation values are $3.76 \mu \mathrm{m}$ and $16.1 \mu \mathrm{m}$, respectively.

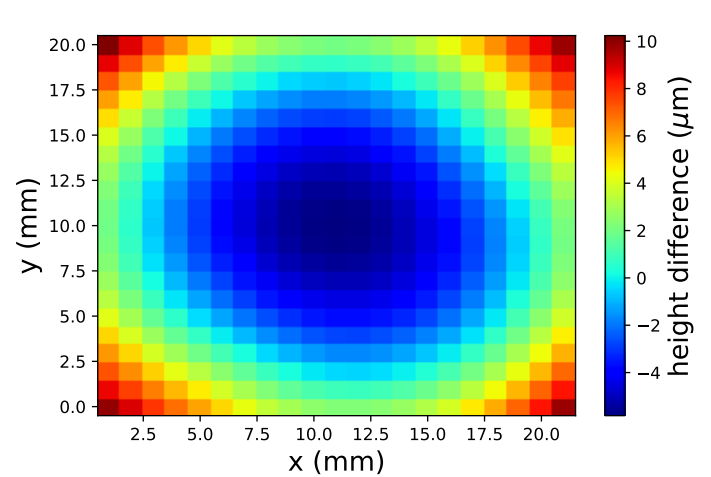

(a) Deformation due to coating.

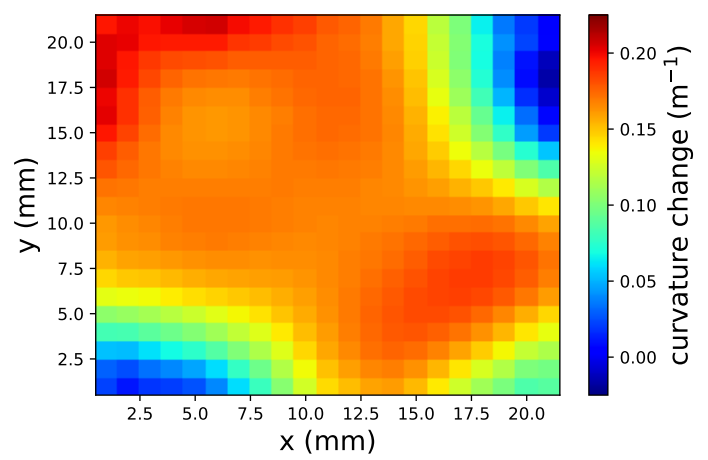

(b) Average curvature change due to coating.

Figure 3.19: Map of height difference (a) and of direction averaged curvature difference (b) of the coated side of a D263M cover glass after coating. For deformation, an about $90 \mathrm{~nm}$ thick chromium film was deposited, and during deposition two areas at the sample boundary were intentionally covered. The height map of the pristine sample has been subtracted from the measured height map. 
As I measured the height maps in two orthogonal directions (named directions 1 and 2), the curvature difference $k_{1}(x, y)$ and $k_{2}(x, y)$ in these two directions can be extracted from them. I obtained the average curvature difference $k(x, y)$ by taking the arithmetic mean of those two curvature maps $k(x, y)=1 / 2\left(k_{1}(x, y)+k_{2}(x, y)\right)$. The average curvature difference $k(x, y)$ after coating is displayed in Fig. $3.19 \mathrm{~b}$. To achieve a non-uniform coating, a significant part of the surface was intentionally covered by clamps during the deposition. At these positions (bottom left and top right) the curvature change is clearly smaller than on the rest of the surface. In addition, to achieve a film thickness gradient, the sample holder was not rotated during deposition.

From the average curvature difference, I estimated the local integrated stress via the Stoney Eq. 1.1. . For an irradiation at a constant fluence of $600 \mathrm{~mJ} / \mathrm{cm}^{2}$ and spotsize of $200 \cdot 200 \mu \mathrm{m}^{2}$, I then translated the integrated stress into a spot density for irradiation (explained below), which led to the irradiation pattern shown in Fig. 3.20a, which I used for irradiation of the non-coated side of the sample with one pulse per position. For illustration, Fig. $3.20 \mathrm{~b}$ shows a microscope image of the irradiated sample surface. Irradiated and non-irradiated areas can be distinguished.

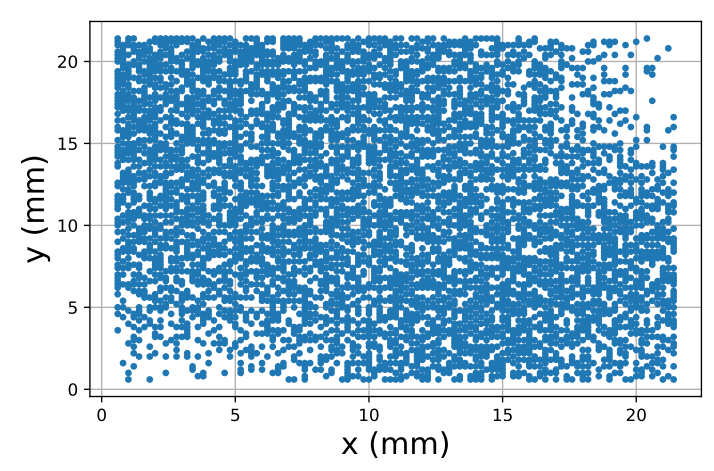

(a) Irradiation positions

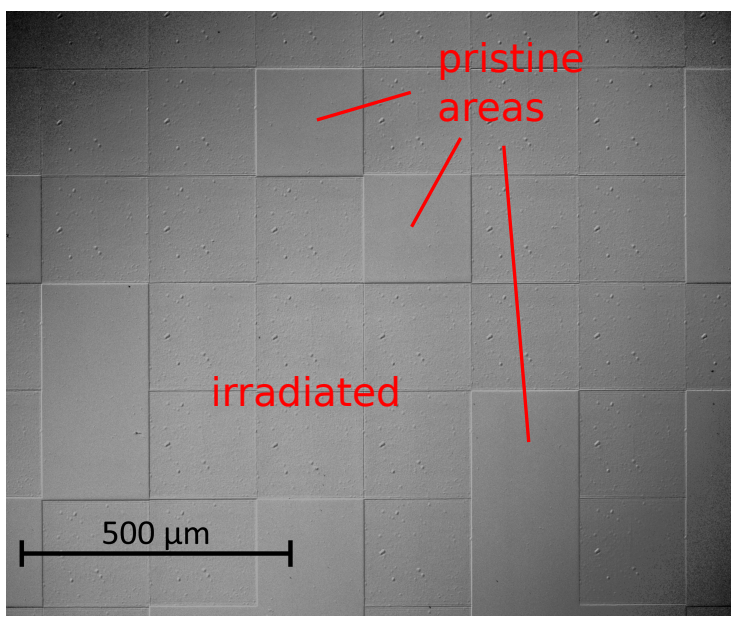

(b) Microscope image of irradiated surface

Figure 3.20: (a) The irradiation pattern for the sample of Fig. 3.19 and Fig. 3.21. (b) A RL microscope image of the irradiated surface taken with DIC. Irradiated and non-irradiated areas can be observed.

For calculation of the irradiation pattern, I virtually divided the measured surface area into square sized cells of $1 \mathrm{~mm}$ edge length. Thus, at a spot size of $200 \mu \mathrm{m}$, the cell could be filled by 25 spots without overlap. I calculated the number of spots per cell with index $i$ via

$$
\begin{aligned}
n_{\text {spots }}^{i} & =25 \cdot r_{A}^{i} \\
& =25 \frac{N_{\text {target }}^{i}}{N_{\text {irr }}} \\
& =\frac{25}{N_{\text {irr }}} \frac{E_{s} t_{s}^{2}}{6\left(1-\nu_{s}\right)} k_{i}
\end{aligned}
$$


Here, $r_{A}^{i}$ is the fraction of the area, that needs to be irradiated, of cell $i, N_{\text {target }}^{i}$ is the desired integrated stress to be induced in the area of cell $i$ and $N_{\text {irr }}$ is the integrated stress that will be induced by irradiation with the chosen fluence. For Eq. 3.5), the desired integrated stress $N_{\text {target }}^{i}$ was approximated by the Stoney Eq. (1.1) with $N=\sigma_{f} \cdot t_{f}$. For this, I measured the sample thickness $t_{s}$ and used the direction averaged curvature difference $k_{i}$ of cell $i$, which was caculated from the deformation of the plate (cf. Fig. 3.19b). Please note that any non-local mechanical effects are neglected by this approximation [17, 18]. The number of spots per cell $n_{\text {spots }}^{i}$ was rounded to a positive integer value and the corresponding amount of spots was randomly distributed inside each virtual cell of $1 \mathrm{~mm}^{2}$ size.

Fig. 3.21a shows the deformation of the coated side of the sample after irradiation. The PV value has been reduced by a factor of 10 to $1.6 \mu \mathrm{m}$ and the RMS value by a factor of 14 to $0.26 \mu \mathrm{m}$ compared to the deformation after coating. This demonstrates the potential of the method for figure correction. Fig. 3.21b shows the curvature change (sign changed for better comparability) of the coated side of the sample induced by irradiation. A comparison with Fig. $3.19 \mathrm{~b}$ shows that the curvature change by the coating has been very well reproduced. I simulated the deformation expected by the figure correction (irradiation) step via the FEM by assuming the deposition of a thin film structured according to the irradiation pattern on the backside surface, corresponding to the non-coated (irradiated) surface in the experiment, of a flat plate. Fig. 3.21c shows the expected deformation after the correction (irradiation). It would have led to a PV value of $0.92 \mu \mathrm{m}$ and a RMS value of $0.16 \mu \mathrm{m}$. Thus, a slightly better correction could have been obtained under perfect conditions. By comparison of the simulated curvature change (Fig. 3.21d) with the obtained curvature change (Fig. $3.21 \mathrm{~b}$ ) it can be concluded that the experimentally obtained integrated stress was slightly smaller than the optimal value. Still, the correction worked very well.

Tab. 3.1 gives an overview of three samples that were deformed by deposition of a $90 \mathrm{~nm}$ thick chromium film and figure corrected the same way as explained above. All samples were coated simultaneously. In the case of sample 3, the example mentioned above, a large area of the surface was covered by clamps during deposition. Therefore, the factor of improvement is lower than in the case of the two other samples. However, for all three samples, the RMS deformation was improved by more than a magnitude.

Table 3.1: Summary of the results for figure correction of three samples. All samples were coated simultaneously with about $90 \mathrm{~nm}$ of chromium. During deposition the sample holder was not rotated. Additionally, in the case of sample 3, the clamps holding the sample were intended to cover a large area of the sample surface.

\begin{tabular}{|c|c|c|c|}
\hline & \multicolumn{2}{|c|}{ RMS deformation $[\mu \mathrm{m}]$} & \\
sample & after coating & after correction & factor of improvement \\
\hline 1 & 3.88 & 0.22 & 18 \\
2 & 3.80 & 0.2 & 19 \\
3 & 3.76 & 0.26 & 14 \\
\hline
\end{tabular}




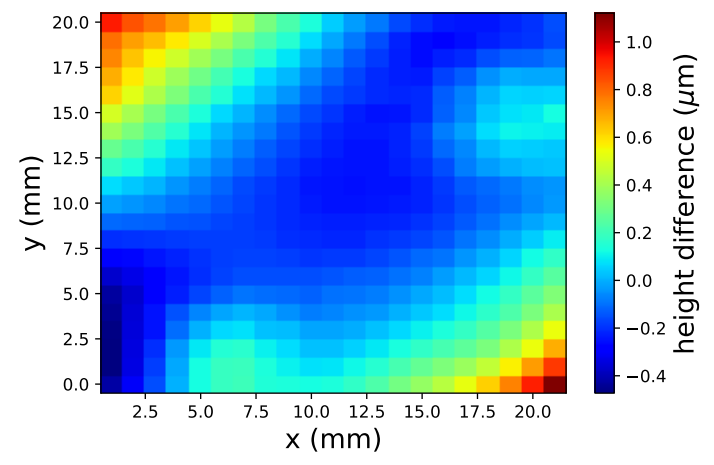

(a) Deformation after figure correction.

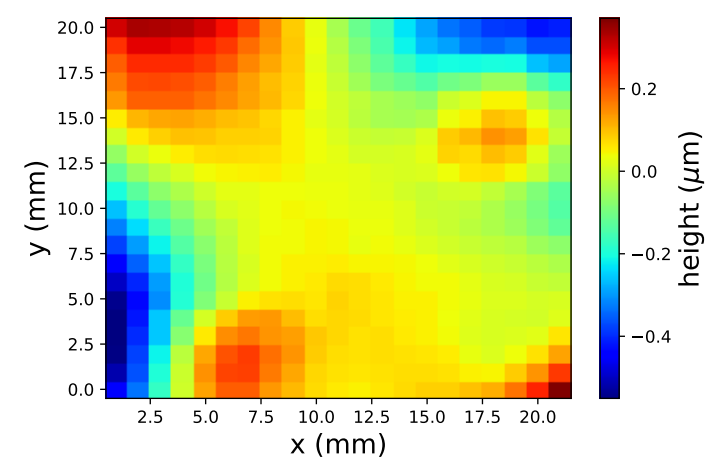

(c) Simulated heightmap after correction.

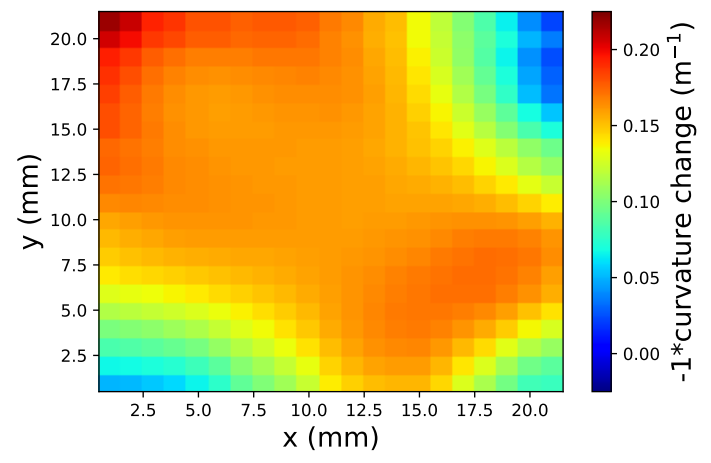

(b) Average curvature change by figure correction.

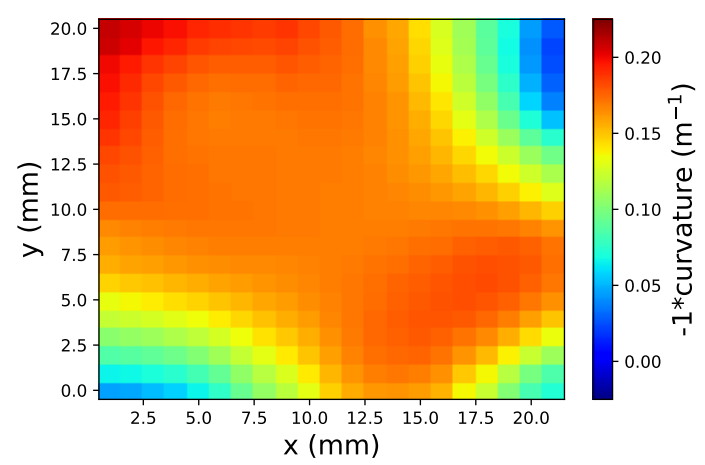

(d) Simulated curvature change.

Figure 3.21: Map of deformation of the sample after the figure correction step (a) and of direction averaged curvature change by the irradiation (b). For irradiation, a square spot with a size of $200 \mu \mathrm{m}$ at a fluence of $600 \mathrm{~mJ} / \mathrm{cm}^{2}$ at one pulse per position was used. In (c), the simulated result of the figure correction step is shown. (d) Shows the simulated curvature change by the applied irradiation pattern. Please note that I inverted the sign of the curvature change in (b) and (d) for better comparison to Fig. $3.19 \mathrm{~b}$ 


\subsection{Discussion}

In section 3.1, I presented experimental results on the deformation of borosilicate cover glasses of Schott D263M via the light of an ArF excimer laser. I demonstrated that the about $210 \mu \mathrm{m}$ thick cover glasses can be significantly deformed via irradiation, and that the deformation can be precisely described by a linear elastic deformation due to a tensile stressed surface layer in the irradiated areas (Figs. 3.4 and 3.5). I estimated the thickness of this stressed surface layer to be in the range of a few hundreds of nanometers (Fig. 3.10) and its integrated stress to be as high as about $250 \mathrm{Nm}^{-1}$ (Fig. 3.6). This integrated stress depends on the laser fluence (Fig. 3.6), but there is no pronounced dependence on the pulse number or the area of the laser spot (Fig. 3.8). The absolute value of the induced curvature change decreases with time for storage in air (Fig. 3.17). Further, I demonstrated that antibiaxial plane stress components can be induced via irradiation with a line pattern of a period of $8 \mu \mathrm{m}$ (Fig. 3.14a). A possible application for such stresses could be a figure correction method. For this, I performed a proof of principle. Here, I discuss the results with regard to the mechanism for stress generation, the reason for the time evolution of the stress, the generation of antibiaxial stresses and the possible application of the stresses.

\subsubsection{The origin of the stress}

A common process in laser beam bending is the temperature gradient mechanism (TGM) [48],[47, Ch. 3.2.1]: Due to heating, plastic deformation and re-solidifying of a surface layer, a tensile stress is developed at the irradiation position. I assume that the TGM is the main cause for the observed surface stress, which is in accordance with the interpretation in [68] for the bending of fused silica sheets via irradiation with an $\mathrm{F}_{2}$ excimer laser.

On the basis of the TGM, the measured step-like dependence of the integrated stress on the fluence in Fig. 3.6 can be qualitatively explained (Fig. 3.22): At fluences below about $100 \mathrm{~mJ} / \mathrm{cm}^{2}$ the absorbed energy is too small to induce a sufficient viscosity decrease of the glass material for plastic deformation and a tensile stress after cooling down. Therefore, no curvature change is induced. At higher fluences a layer of 'molten' and re-solidified material develops at the surface, which exhibits a tensile stress. The thickness of this layer increases with increasing fluence because of the decay of the light intensity inside the material and thermal conduction. However, at fluences above the ablation threshold, the material closest to the surface is removed by laser ablation. Thus, the thickness of the stressed surface layer is limited and a step-like dependence of the integrated stress in dependence on the fluence is observed, as in Fig. 3.6.

The above interpretation of the experimental results is supported by the measurements of the change in surface height of the irradiated area with respect to the non-irradiated area (Fig. 3.7). The increase of the surface height for irradiation with a fluence in between 150 and $250 \mathrm{~mJ} / \mathrm{cm}^{2}$ could be a result of melt expansion and 


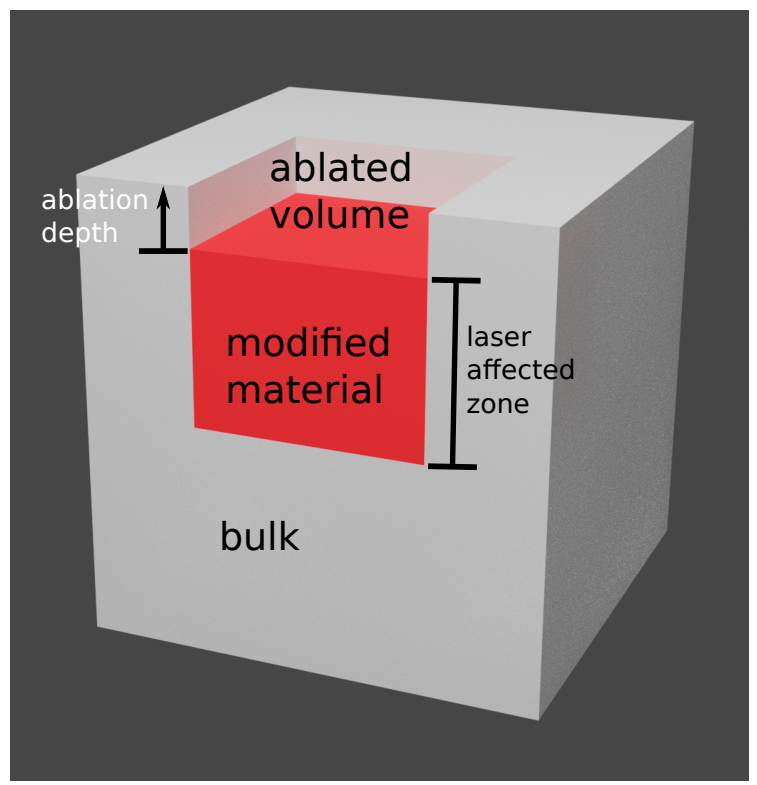

Figure 3.22: Scheme of a section of the sample after irradiation. In the irradiated area, the material is ablated to certain depth. Below the ablated area, the material is heated to such a high temperature that (partial) relaxation of the thermal stresses occurs. During cooling the material contracts and a tensile stress is generated inside the modified material.

indicates that no major ablation took place. In contrast, the decrease in surface height at fluences of $360 \mathrm{~mJ} / \mathrm{cm}^{2}$ and above indicates the removal of material by laser ablation. Therefore, the onset of laser ablation happens roughly in the middle of the step-like region in Fig. 3.6, which is exactly the position one would expect it to happen following the above interpretation. For ablation of Schott D263T eco, a threshold fluence of about $400 \mathrm{~mJ} / \mathrm{cm}^{2}$ was reported [127, Tab. 5.8]. Additionally, for Schott BK7, another borosilicate glass, a threshold fluence of $260(50) \mathrm{mJ} / \mathrm{cm}^{2}$ for $\mathrm{ArF}$ excimer irradiation was experimentally determined [65]. These values agree well with my rough estimation for D263M.

If the sample is etched after irradiation, the stressed surface layer is removed and the curvature change returns to zero, as is demonstrated in Fig. 3.2, 3.6 and 3.9a. As described in section 3.1.3, etching also allowed to estimate the maximum induced stress and the extent of the stressed surface layer. I measured a maximum stress of about $400 \mathrm{MPa}$ and a thickness of about $650 \mathrm{~nm}$ for a fluence of $1 \mathrm{~J} / \mathrm{cm}^{2}$. For the stress, a rough theoretical estimate based on material parameters can be achieved under the assumption that the glass behaves purely elastic below the transformation temperature $T_{g}=557^{\circ} \mathrm{C}[128$ and exhibits a sufficiently small viscosity above it, such that any stresses relax virtually instantaneously above $T_{g}$ :

$$
\begin{aligned}
\sigma & =\frac{E}{1-\nu} \epsilon_{\mathrm{th}} \\
& =\frac{E}{1-\nu} \alpha\left(T_{g}-T_{0}\right) \\
& =354 \mathrm{MPa} .
\end{aligned}
$$

Here, I used the biaxial modulus $E /(1-\nu)$, the thermal strain $\epsilon_{\mathrm{th}}=\alpha\left(T_{g}-T_{0}\right)$, 
the linear thermal expansion coefficient $\alpha$ and the temperature $T_{0}=20^{\circ} \mathrm{C}$ of the sample/the laboratory during irradiation. For the material parameters $E, \nu, \alpha$ and $T_{g}$, I used the values in Tab. A.1 in the appendix. Please note that the value of $\alpha$ is only specified in the range between 20 and $300{ }^{\circ} \mathrm{C}$. The theoretical value in Eq. (3.8) fits well to the experimentally estimated value of $400 \mathrm{MPa}$. This is another argument for the hypotheses of melting induced stresses by the TGM. For the irradiation of fused silica with an $\mathrm{F}_{2}$ laser, an average tensile stress of about $80 \mathrm{MPa}$ was observed inside the stressed surface layer after irradiation [68]. The smaller value compared to D263M can be explained by the much smaller coefficient of thermal expansion of fused silica. According to Eq. (3.7), $\alpha \approx 0.5 \cdot 10^{-6} \mathrm{~K}^{-1}$ and $T_{g} \approx 1150^{\circ} \mathrm{C}$, a stress value of $49 \mathrm{MPa}$ would be expected. This value also is in good agreement with the experimental value of $80 \mathrm{MPa}$.

According to the above explanation of the step-like dependence of the integrated stress on the fluence, the measured thickness of $650 \mathrm{~nm}$ of the stressed surface layer can be considered as the maximum possible thickness because the fluence of $1 \mathrm{~J} / \mathrm{cm}^{2}$, at which this value was measured, is well above the step region in Fig. 3.6. This thickness can be compared to the thermal diffusion length

$$
L_{t h}=\sqrt{2 \kappa \tau_{L}}=\sqrt{2 \frac{\lambda_{c} \tau_{L}}{\rho_{m} C_{p}}} \approx 150 \mathrm{~nm}
$$

as defined in [129]. Here, $\kappa=\lambda_{c} /\left(\rho_{m} \cdot C_{p}\right)$ (see e. g. [100, p. 207]) is the thermal diffusivity of Schott D263, $\lambda_{c}$ the thermal conductivity, $\rho_{m}$ the mass density, $C_{p}$ the heat capacity at constant pressure and $\tau_{L} \approx 20$ ns the duration of the laser pulse. For the material properties of D263 the values in table A.1 in the appendix were used. The calculated thermal diffusion length is, although of the same order of magnitude, clearly smaller than the measured thickness of the laser affected zone. This discrepancy could be explained by a penetration depth of the incident laser light of a few hundred nanometers 2 . Further, the discrepancy demonstrates that valuable information about the ablation process can be obtained by measurement of the stress distribution inside an ablated surface. For irradiation of fused silica with an $\mathrm{F}_{2}$ laser, a thickness of the stressed surface layer of about $275 \mathrm{~nm}$ was measured [68]. This value is of the same order of magnitude as my result of about $650 \mathrm{~nm}$ thickness. Further, it was noted that the thickness does not depend on the laser fluence [68], which supports the same assumption I made above.

In Fig. 3.9c it is shown that the etch rate in a $\mathrm{KOH}$ solution at $80^{\circ} \mathrm{C}$ is apparently faster inside the irradiated area than in the non-irradiated area. I mentioned above that the height of the irradiated surface increases if ablation is not present. Thus, the mass density of the material is lowered by irradiation. It is reasonable to assume that this tensile stressed material with a lower density is etched faster than the pristine material. If the sample is etched before irradiation, the integrated stress as a function of the fluence still follows almost the same curve as for the pristine samples, which is shown in Fig. 3.6. This is in accordance with the above interpretation of the stress generation because etching is supposed to just thinning the sample.

\footnotetext{
${ }^{2}$ Please note that for glasses there can be a difference between the linear absorption according to the Lambert-Beer law and the effective absorption of the excimer laser light $130-132$.
} 
However, etching could also change the surface texture, which could explain the slight deviation from the curve of the pristine samples in Fig. 3.6.

I observed no pronounced influence of the pulse number per irradiation position on the integrated stress (cf. Fig. 3.8a). The same was reported in [68 for the irradiation of fused silica and would be expected from the above interpretation of the stress generation because, at the low repetition rates $(\leqslant 10 \mathrm{~Hz})$ applied, the glass presumably cools down in between individual pulses 3 . I. e. there is no cumulative heating effect and the glass is just melted again and again during each pulse, leading to the same stress and thickness of the re-solidified layer. However, there could be a difference between the first and second pulse because the first pulse hits a pristine surface. For all three fluences in Fig. 3.8a we see an increase in the integrated stress from the first to the second pulse. This could be a hint to an incubation effect, namely a change in absorption of the material due to defects generated by the laser irradiation [133, Secs. 12.3 and 13.4.2]. In laser ablation, incubation manifests itself by a decrease in the ablation threshold with increasing number of pulses 133 , Sec. 12.3]. Since incubation has been observed for excimer laser ablation of Er/Yb-doped borosilicate glass [64] and fused silica [62, 63], it would be no surprise if it would happen in the present case as well.

It is well known that the fast cooling of glasses from a temperature regime of low viscosity can give rise to a permanent compressive stress inside a surface near region due to the temperature gradients during the cooling process (see for example 134 , Ch. 9.4.4]). Thus, on the first glimpse another mechanism to explain our experimental results could be that the sample surface is compressively stressed due to the manufacturing process and this stress is reduced locally by irradiation. Therefore, a relatively tensile stress would be produced locally at the sample surface that would lead to the deformations shown in Fig. 3.4 and 3.5. This hypothesis of a compressively stressed surface layer could also explain the behavior of the integrated stress in dependence on the fluence shown in Fig. 3.6 for pristine samples and samples etched after irradiation: Increasing fluence leads to a more pronounced stress reduction, and at a fluence of about $500 \mathrm{mJcm}^{-2}$ the stress has been been reduced through the whole thickness of the stressed layer. Therefore, no further increase of the integrated stress will be observed. And via etching, the stressed surface layer is removed on the whole sample surface. However, this alternative model fails at explaining why the samples behave almost the same way when irradiated after etching as without etching. One would expect that if the compressively stressed surface layer is removed due to etching, the subsequent irradiation would lead to a totally different behavior of the integrated stress on the fluence. This is obviously not the case. Additionally, a strong dependence of the integrated stress on the pulse number at small fluences would be expected. This is also in direct contradiction to the experimental results of Fig. 3.8a, where no pronounced dependence on the pulse number can be seen. Therefore, this alternative model, based on a compressively stressed surface layer, for explanation of my experimental results is presumably wrong.

\footnotetext{
${ }^{3}$ See for example the calculations made in 84
} 


\subsubsection{Stability of the integrated stress and crack formation}

In the preceding section, I argue that the observed stressed surface layer is generated by the TGM, namely via thermal contraction of the laser heated surface layer. Apparently, the stress is not stable over time. It is demonstrated in Fig. 3.17 that the generated surface stress decreases over time in ambient air at room temperature and at $100^{\circ} \mathrm{C}$. Further, Fig. $3.17 \mathrm{~b}$ shows that the surface stress is less stable at increased temperature. These findings raise the question of the underlying processes responsible for the decline in absolute integrated stress.

It is interesting to compare the experimentally estimated value of the maximum stress of about $400 \mathrm{MPa}$ (Fig. 3.10) to the strength (maximum stress without rupture) of the bulk material. A typical tensile strength of a glass in a water-free environment is about $70 \mathrm{MPa}$, and the maximum design strength for use in ambient air is about $20 \mathrm{MPa}$ [100, p. 363]. Thus, the observed stress is an order of magnitude larger than the expected strength of the glass. A similar, although not as much pronounced, discrepancy between surface stress and material strength has been observed in 68 for fused silica ablated with an $\mathrm{F}_{2}$ excimer laser. The authors speculate that there is some strengthening mechanism of the surface material due to the laser irradiation. However, it could be more appropriate to compare the measured stress values to the strength of thin films of the same material because of the sub-micron thickness of the stressed surface layer. For example, for $1 \mu \mathrm{m}$ thick beams of amorphous $\mathrm{SiO}_{2}$, tensile strength values of 364(57) [135], 426(63) [136 or $810(60) \mathrm{MPa}$ [137] have been reported. They are an order of magnitude larger than the bulk values, which is explained in [135] by the necessarily smaller size of surface flaws than for a macroscopic sample. This could indicate that the short-time annealing or ablation leads to a reduction of the flaw size of the surface in the irradiated area. However, in 68 the authors argue against such a process by noting that optical polishing induces defects inside the surface. Therefore, further study is necessary to explain the large strength of the stressed surface layer of glasses after excimer laser irradiation.

In 137] it is demonstrated for a thin film of $\mathrm{SiO}_{2}$ that, although the strength of this film is significantly higher than in the bulk, the fracture toughness (resistance to crack propagation) is the same in both cases. This means that any flaws in the thin film material behave similarly as in the bulk and allows for sub critical crack growth (SCCG, a slow crack propagation process, see e. g. [138]) as a possible, but highly speculative, explanation for the decrease in absolute integrated stress with time. In certain regimes of SCCG, the crack velocity increases with increasing relative humidity in a gaseous enviroment and with increasing temperature 138 140]. Indeed, for thin films of $\mathrm{SiO}_{2}$, a smaller strength and fracture toughness have been observed for testing in air than in vacuum, which was explained by humidity [141]. As I stored my samples in ambient air, humidity might have had an influence on them. Additionally, I demonstrated a faster decrease of the integrated stress at $100{ }^{\circ} \mathrm{C}$ than at room temperature in Fig. 3.17. These observations could be a hint to SCCG of many nano-sized cracks inside the stressed surface layer as an explanation for the decrease in integrated stress with time. 
Indeed, I observed cracks in the irradiated area after etching, both in the case of the quadratic spot (Fig. 3.11) and for the spot with a line pattern (Fig. 3.16). Much less often I also observed cracks after treatment in water. In both cases, these cracks are definitely caused by the irradiation as they only appear inside the laser spots and are sharply bounded by the borders of it. In laser irradiation of glasses, crack formation is occasionally encountered $[61,63,69,70]$ and in $[70]$ it is associated with the tensile stresses inside the solidified molten layer. Surprisingly, in my case the cracks become more pronounced with the ongoing etching procedure and do not vanish when the integrated stress does. Thus, they seem to be also located inside the pristine material below the solidified molten layer. Therefore, they could be, at least partly, a different phenomenon than the above assumed cracks inside the solidified molten layer. This interpretation is supported by the absence of cracks after longterm storage or annealing of the samples (Sec. 3.1.5). In [69] it has been argued that cracks are also formed during laser heating of the surface by the thermal expansion of the heated material and the resulting tensile stresses at the bottom (the border between heated and pristine material) of the laser affected zone. Such a mechanism could explain the cracks observed inside the pristine material.

An alternative or additional effect on the evolution of the generated stresses could be a change of the elastic stress by water absorption. For vacuum deposited $\mathrm{SiO}_{2}$ films, water uptake has been demonstrated [142], and it has been suspected that it has a significant influence on the film stress [143].

For further investigation of the instability of the generated surface stress and the mechanisms for crack formation, it might be enlightening to have a closer look on the laser affected material in a high resolution cross-sectional view via TEM, SEM or AFM and to study the decrease of the integrated stress in dependence on the humidity of the gaseous environment.

\subsubsection{Generation of antibiaxial stress components}

In section 3.1.4, I demonstrated that an antibiaxial plane stress component can be generated via irradiation with a line pattern with a period of $8 \mu \mathrm{m}$ and a duty cycle of 0.5. To the best of my knowledge, a similar result has not been reported, yet. Kakehata et al. presented anisotropic surface stresses for laser-induced periodic surface structures (LIPSS) on the surface of yttria-stabilized zirconia ceramics [144]. However, they concluded that the stress was generated by a phase change and domain switching. Opposite to my experiments, they observed a larger magnitude of stress across the lines than along them. Thus, presumably the governing mechanisms are different in their experiments and in mine.

In [19], an anisotropic surface stress was generated by oxidizing a silicon surface and subsequently lithographically patterning into fine parallel lines. The curvature ratio $k_{s} / k_{p}$ of such a line pattern is a function of the aspect ratio (height divided by width) of the stressed lines [3, 19]. Therefore, I assume that a similar mechanism is responsible in my case for the anisotropic deformation. This hypothesis is supported by the measured decrease of the ratio $k_{s} / k_{p}$ of curvature change $k_{s}$ across and $k_{p}$ 
along the lines with increasing structure depth (Fig. 3.15). However, in [19] only the ridges (the lines) are prestressed, but in my case one would naively assume that the highest integrated stress is in the trenches of the line structure, because, in case of a perfect mask projection, these are the irradiated areas. This assumption has been addressed in Fig. 3.14b where the integrated stress is plotted against the calculated fluence inside the trenches. Although for calculation of the integrated stress the line profile of the laser spot has been neglected, the integrated stress saturates at a value of about $240 \mathrm{Nm}^{-1}$, which corresponds surprisingly well to the saturation value in Fig. 3.6 of about $250 \mathrm{Nm}^{-1}$. If only the trenches would be prestressed, I would expect half of this value for the integrated stress. Thus, at higher fluences and higher pulse numbers, the irradiation conditions led to a significantly stressed surface layer also on the ridges of the line structure. This is probably caused by a low value of the Modulation Transfer Function of the optical setup. For a higher contrast, spherical aberrations should be reduced by changing the lens to an aspheric or achromatic one or decreasing the size of the mask. Another possible contribution to a stressed surface layer on the ridges can be observed in [65]. In this publication the line patterning of the borosilicate glass BK7 by an $\mathrm{ArF}$ excimer laser is investigated. It is demonstrated that a considerable amount of molten material is deposited on the ridges of the lines during patterning. If this material exhibits a good adhesion to the underlying surface, it could as well contribute to the surface stress on the ridges.

For my case, a simple analytical model can be deduced by combination of the model of Wikström et al. (1.4) [3], for the anisotropic contribution of the ridges, with the Stoney Eq. (1.1) [11], for the isotropic contribution of the trenches:

$$
\begin{aligned}
\mathbf{k} & =\underbrace{-\frac{6\left(1-\nu_{s}^{2}\right)}{E_{s}} \frac{t_{f}}{t_{s}^{2}} \sigma \frac{b}{d} \mathbf{P}_{s}^{-1}\left(\begin{array}{c}
1-\chi(\rho) \nu_{f} \\
1-\chi(\rho) \\
0
\end{array}\right)}_{\text {Wikström }} \underbrace{-\frac{6\left(1-\nu_{s}\right)}{E_{s}} \frac{t_{f}}{t_{s}^{2}}}_{\text {Stoney }} \sigma \underbrace{\left(1-\frac{b}{d}\right)}_{\text {fraction of trenches }}\left(\begin{array}{l}
1 \\
1 \\
0
\end{array}\right) \\
& =-\frac{6\left(1-\nu_{s}\right)}{E_{s}} \frac{t_{f}}{t_{s}^{2}} \sigma\left[\left(1+\nu_{s}\right) \frac{b}{d} \mathbf{P}_{s}^{-1}\left(\begin{array}{c}
1-\chi(\rho) \nu_{f} \\
1-\chi(\rho) \\
0
\end{array}\right)+\left(1-\frac{b}{d}\right)\left(\begin{array}{l}
1 \\
1 \\
0
\end{array}\right)\right]
\end{aligned}
$$

I introduced the minus signs to account for the the fact that I measured the curvature of the non-irradiated sample side. The curvature ratio $k_{s} / k_{p}$, calculated by the theory of Wikström et al. (1.4) and by the above combination (3.11), is plotted in Fig. 3.15 as a function of the structure depth for a line pattern with a period of $d=8 \mu \mathrm{m}$ and a duty cycle of $b / d=0.5$. For both equations, the curvature ratio decreases with increasing structure depth. However, the above theory (3.11) results in higher values of the curvature ratio than the basic theory of Wikström et al. A comparison with the experimental data shows that for smaller depths, the curvature ratios agree well with the extended theoretical description (3.11), while the two points of largest depth lie between both theoretical curves. Please note that a change in the width of the ridges in between the samples is not considered in Fig. 3.15. Also, the theory of Wikström et al. does not consider the film stress distribution in direction normal to the surface before patterning. Additionally, for the sample with the smallest curvature ratio, I observed cracks running parallel to the lines after cleaning (Fig. 3.16b). If they were there also before the cleaning 
procedure, when the curvature was measured, according to the Wikström theory, they would have increased the effective structure depth but would have only slightly increased the measured depth. The decreased curvature ratio after cleaning (cf. Figs. 3.16c and 3.16d) could therefore also be explained via crack growth. Thus, from Fig. 3.15 one might suspect that Eq. (3.11), the combination of the theories of Wikström et al. and Stoney, could be a good description of the experimental results.

This assumption should be verified by an improved experiment. A better homogeneity of the structure depth over the laser spot could be achieved by use of a smaller mask area and an achromatic projection lens instead of a spherical one. The influence of the debris could be reduced by irradiation in vacuo or in helium atmosphere for a more precise measurement of the structure depth.

The finding of a strong anisotropy of the surface-near stresses in case of a laser generated micro-structure is, in my opinion, a valuable result for understanding the side effects in laser structuring. My results and further investigation could help to understand and predict crack orientations, strength reduction and deformation of thin or small parts.

\subsubsection{A method for figure correction?}

In section 3.1.6, I made a proof of principle for a figure correction method by a laser induced stressed surface layer. Although an approximate method for calculation of the necessary integrated stress field was applied, the RMS height error could be reduced by more than a magnitude. Therefore, the factor of figure improvement is comparable to the factors obtained in [36] for correction of Si substrates by ion implantation, which were deformed by a magnetron sputtered chromium film. Still, there is a significant difference between the simulated and the achieved surface topography after correction (Figs. 3.21c and 3.21a). This could be caused by errors in the generated integrated stress or by a non-uniform sample thickness.

A possible workflow for the proposed method for figure correction is shown in Fig. 3.23. The process could be used to correct the deformation of optical parts based on a thin glass substrate and some functional film on the front side of the substrate. A correction of the shape of the front side of the substrate might be needed due to inaccuracies in the manufacturing process or the deposition of a stressed functional film (e. g. a mirror coating). In either case, the deviation of the front surface is measured and an irradiation pattern for the backside surface is calculated. For calculation of the irradiation pattern, the field of integrated stress, that is to be induced, must be known. In [38], different analytic approaches for calculation of the integrated-stress field are given for the case of a round plate. The authors show that if also non-equibiaxial stress components are considered, a perfect correction can be achieved. The authors of [38] did not consider non-local mechanical effect:4

\footnotetext{
${ }^{4}$ I conclude this statement from the observation that Eqs. (9) of 38, which are supposed to be an exact integrated-stress field, are a set of local equations. Further, a similiar set of equations has been derived in [145] (Eqs. (34)) explicitly for the case of uniform strains inside the film. Eqs. (34) did not follow for an arbitrary (lateral) strain distribution inside the film.
} 
as they were described in 17,18$]$. However, in Figs. $3.4 \mathrm{~d}$ and $3.5 \mathrm{~d}$ of this thesis, a non-zero curvature in the non-irradiated areas can be observed and is confirmed by simulation. This demonstrates the influence of non-local mechanical effects. Therefore, depending on the application, such effects should also be considered in the calculation of the integrated-stress field. Irradiation could be performed with an ArF excimer laser, as in my experiments. Subsequently, the induced stress has to be stabilized by an annealing procedure and the success of the correction step should be controlled.

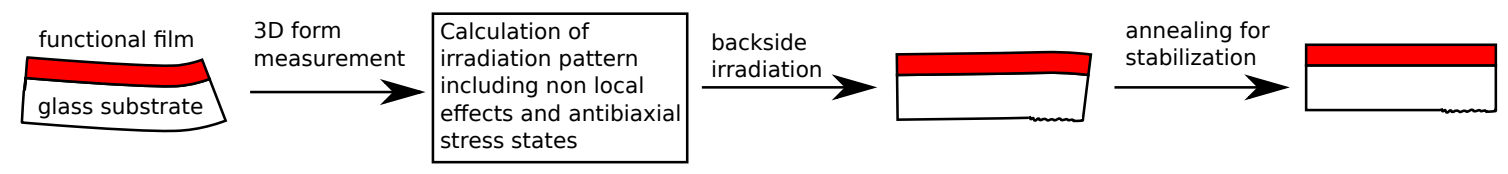

Figure 3.23: A proposed workflow for figure correction of glass substrates by laser induced stresses. Based on a measurement of the deviation of the deformed part, an irradiation pattern for the non-functional side of the part is calculated. By irradiation, the deformation is over compensated because the subsequent stabilization step causes a partial relaxation of the laser induced stress.

This process has some advantages compared to similar figure correction methods. As a laser process, it can be easily adopted to each sample and is free of any hazardous chemicals. Although excimer lasers have moderate repetition rates, I expect it to be a fast process because of the high pulse energy of excimer lasers of up to a few hundred millijoules at $193 \mathrm{~nm}$ wavelength ${ }^{5}$. I. E. an area of a few tens square millimeters could be processed at a fluence of $1 \mathrm{~J} / \mathrm{cm}^{2}$ with a single pulse. If a flattop beam profile is used, which is easily accomplished with an excimer laser, only one calibration curve is needed for all spot sizes. I expect this to be much more complicated in the case of a non-uniform intensity distribution inside the beam or in case of non-linear absorption, like it was used in [35]. The optical setup shown in Fig. 2.1 is simple, robust and exhibits a large depth of focus. Thus, handling of the setup is easy, and in many applications, adjustment of the image plane would not be necessary during irradiation of a slightly curved surface. As I demonstrated in section 3.1.4, generation of antibiaxial plane stress components is also possible. This extends the range of correctable deformations significantly [38].

However, there are also some serious disadvantages of this proposed process. Most problematic is the non-stability of the induced stresses, which impedes a precise long-term correction. I therefore suggest to stabilize the stress by an annealing step after irradiation. Annealing of samples after film deposition is a common procedure in thin film technology [9, 22, 23], and an annealing step is also needed in figure correction by ion implantation [36]. But it is still unclear if annealing leads to a stabilization, and if the expected decrease in absolute stress during annealing is precisely reproducible. Additionally, even if the stress can be stabilized for use at room temperature, it could still be quite sensitive to higher application temperatures, thereby reducing the utility of the method. If the instability of the induced stresses is caused by their large magnitude, another approach for obtaining a stable

\footnotetext{
${ }^{5}$ Coherent COMPex 201[56]: $400 \mathrm{~mJ}$ at $193 \mathrm{~nm}$.
} 
stress could be to irradiate the sample when it is already at an increased temperature. A raised temperature of the sample surface would lead to a smaller elastic thermal contraction of the laser-molten material during the cool down and thus to a smaller value of absolute stress (cf. Eq. (3.7) for the thermal stress) [69].

Because I observed crack formation, the strength of the irradiated samples after irradiation and long-term use is another serious issue. Nano- or microcracks may lead to a reduced strength and subsequently to failure of the part under everyday usage. If failure happens in a space application, the result would be catastrophic because exchange of the broken part is impossible. Thus, extensive tests on the strength and fatigue of figure corrected parts have to be done before the method can be commercially applied.

Another disadvantage is the limitation to generation of tensile stresses. Thereby, surface figure errors can only be corrected in one direction. This is fine if the surface error is the result of a tensile stressed functional film deposited on the front side of the sample. But in many other cases it might exclude the method. It has been reported that a laser induced shockwave can also lead to a permanent bending of the sample in direction away from the laser [45], while the TGM leads to a bending in direction towards the laser. Thus, by changing the laser system from a nanosecond excimer laser to a picosecond or femtosecond laser system, both signs of surface plane stress might be accessible. In this case, a tensile stress could still be produced by cumulative heating with many pulses.

Another extension of the above method to compressive plane stresses, and actually also an alternative to it, is to deposit a stressed film on the backside sample surface and to structure it via laser ablation for achieving a certain elastic deformation of the sample. This approach is mentioned in [45] for correction purposes in microelectromechanical systems or microoptoelectromechanical systems and has been patented [44. It has also been studied for the figure correction of silicon substrates via femtosecond laser ablation of a grown oxide film on the backside of the substrate [46].

In my case, a film of silicon suboxide, deposited by thermal evaporation of silicon monoxide, could presumably be used for this approach. For deposition at low vapor pressure and high deposition rate, the film develops a tensile stress on a glass substrate [146]. However, at high vapor pressure and low deposition rate, a compressive film stress is generated [146]. In a preliminary experiment ${ }^{6}$, I measured an average compressive film stress of less than $-200 \mathrm{MPa}$ for $70 \mathrm{~nm}$ of silicon suboxide on D263M deposited at a background pressure of about $1 \cdot 10^{-5} \mathrm{mbar}$, a deposition rate of about $0.5 \mathrm{~nm} / \mathrm{s}$ and a temperature of about $260^{\circ} \mathrm{C}$. The film was still highly absorbing in the ultraviolet spectral region and could be well ablated in front side orientation with the light of an ArF excimer laser 7 .

A possible work flow for this process is sketched in Fig. 3.24. In addition to the one of Fig. 3.23, the form measurement is first used to estimate the thickness of silicon suboxide needed. After deposition of the silicon suboxide, the second form

\footnotetext{
${ }^{6}$ Deposition done by Lukas Janos Richter (IFNano).

${ }^{7}$ Ablation test performed by Lukas Janos Richter (IFNano).
} 
measurement is used to calculate the integrated stress of the film. Based on this result, the irradiation pattern is calculated. At each location, the silicon suboxide film can be removed to a certain depth by a certain amount of pulses to precisely adjust the integrated stress at this position. If it is totally removed at a location, a tensile stress can be (and probably will be unavoidably) induced inside the cleared glass surface. This stress will need to be stabilized, as discussed above.

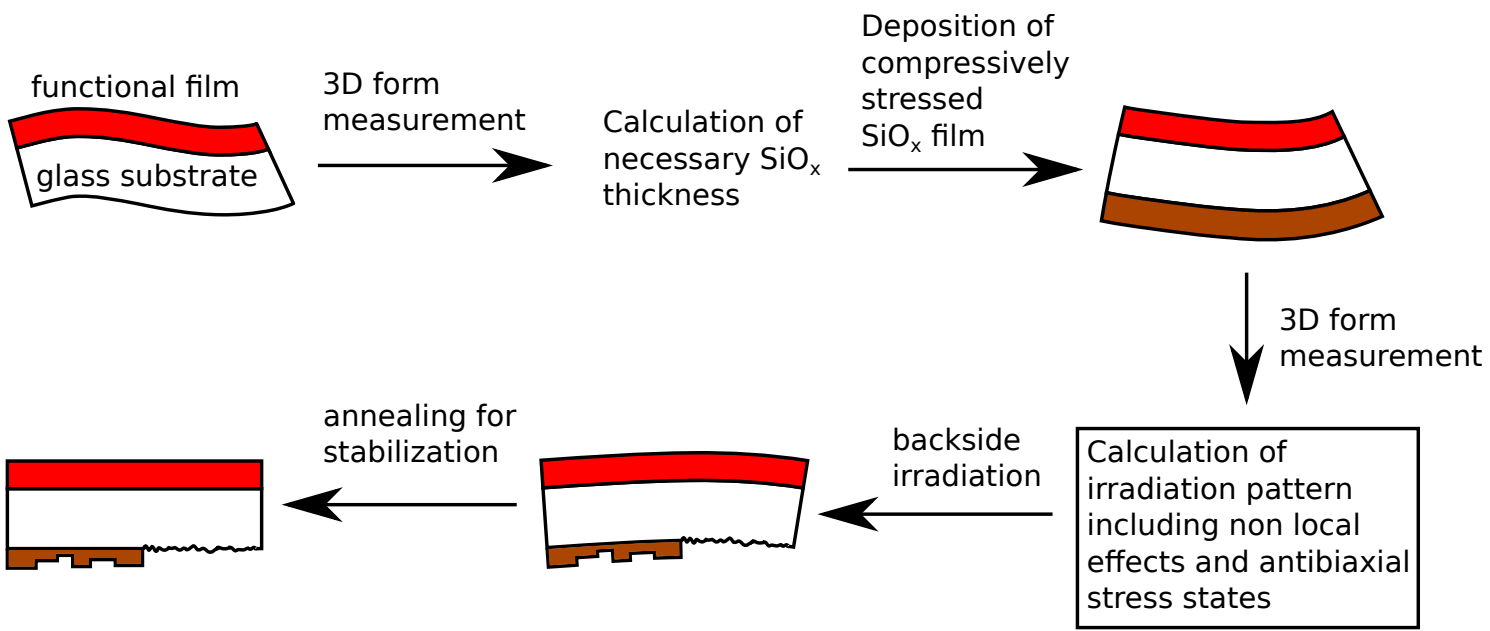

Figure 3.24: A possible work flow for figure correction of glass substrates. By use of a compressively stressed silicon suboxide film, also convex deformations of the front surface can be compensated. 



\section{Chapter 4}

\section{Theoretical considerations for the linear viscoelastic deformation of plates}

In the preceding chapter, I considered the deformation of plates of D263M by a thin stressed layer at the surface, that was generated by irradiation with an ArF excimer laser. As demonstrated by the etching experiments (cf. Sec. 3.1.3), the deformation was elastic. However, at increased temperature (decreased viscosity), glasses show viscoelastic behavior [118, Ch. 3], and in Ch. 5I report on the viscoelastic deformation of vitreous silica substrates by a laser patterned film of $\mathrm{SiO}_{x}$, for which I applied similar methods as in the preceding chapter. The current chapter is supposed to establish some background on the linear viscoelastic behavior of the substrates for a better understanding and interpretation of the experimental results. I make the transition from the elastic case to the viscoelastic case in Sec. 4.1, where I use the viscoelastic analogy to derive some equations for the curvature evolution due to a stressed film out of known elastic equations. Subsequently, in Sec. 4.2, I make use of the linear viscoelastic FE simulation to consider the gravitational deformation of the substrates.

\subsection{Deformation by a stressed film}

The plastic or viscous deformation of substrates due to a stressed film is a common phenomenon [74 78], and a few approaches were made to describe the behavior in case of a continuous film [75, 79 81, 147]. In [79], a numerical model was used to estimate the influence of the thickness ratio $t_{f} / t_{s}$ of film and substrate, respectively, on the curvature evolution of a film-substrate system for the case of maxwellian stress relaxation. In [75], the curvature evolution of a silicon substrate was modeled by introduction of a yield stress. In [147], the stress evolution in film and substrate are calculated by the viscoelastic analogy for the cases of viscoelastic behavior of the film or the substrate. Unfortunately, the curvature evolution is not considered. The stress evolution is also considered in [80] from a quite universal perspective and 
a complicated equation, combining the rate of stress change in the film with the rate of curvature change in the substrate, is given. And in [81 a more practical equation for the rate of curvature change is calculated.

However, to the best of my knowledge, a compilation of simple, closed form equations for the curvature evolution of film-substrate systems with linear viscoleastic deformation of the substrate is still missing. Plus, the case of non-continuous films on linear viscoelastic substrates has, again to the best of my knowledge, not been studied, yet. However, such simple, although approximate, equations are, in my opinion, important to achieve an impression on the overall behavior and dependencies of the curvature evolution. This impression is important for interpretation of experimental results and estimation purposes. Therefore, it is the goal of this section to give an analytical impression for the case of a closed film and a line structured film. Although Eqs. 4.7), 4.19), 4.28) and (4.35), that are derived in this section, might also be deduced from [81] or [80], I followed a path based on existing approximate solutions for the purely elastic case via the viscoelastic analogy.

The viscoelastic analogy can be used to achieve an analytic impression of the viscoelastic behavior of mechanical systems by relatively little effort [118, Chap. 7]. For that, only an elastic solution is needed. In this section, the viscoelastic analogy for the Stoney Eq. (1.1), the equation of Brenner and Senderoff (1.2) and the equation of Wikström et al. (1.4) are calculated. I believe that these equations give valuable insights in the behavior of viscoelastic substrates when deformed by the stress of an elastic film or by a film with exponential stress decay.

For the calculation, two important simplifications are made. First, it is assumed that the shear relaxation modulus can be described by the equation $[118 \text {, Eq. (7.31) }]^{1}$

$$
G_{1}(t)=2 G_{0} \exp (-t / \tau)
$$

with time $t$, instantaneous shear modulus $G_{0}$ and relaxation time $\tau=\eta / G_{0}$, where $\eta$ is the Newtonian viscosity. Thus, delayed elasticity is neglected and the viscous behavior is assumed to be as simple as possible. Second, dilatational relaxation is neglected.

Under these assumptions, the calculation of the viscoelastic analogy is performed by the following scheme [118, Chap. 7]:

1. Calculate the Laplace transform $\mathcal{L}[f(t)](p)$ of the elastic solution $f(t)$ with respect to the parameter $v=t / \tau$. The transform parameter is denoted by $p$.

2. In $\mathcal{L}(p)$, replace the Young's modulus $E$ and the Poisson's number $\nu$ by 118 , Eq. (7.81)]

$$
\mathcal{M}=\frac{E p}{p+2(1+\nu) / 3}
$$

\footnotetext{
${ }^{1}$ The shear relaxation modulus $G_{1}(t)$ defined here and the shear modulus $G(t)$ defined in section 2.8.3 differ by a factor of two. This is because of different definitions in [118] and [40], respectively. This conflict does not influence the value of the instantaneous shear modulus $G_{0}$.
} 
and 118 , Eq. (7.82)]

$$
\mathcal{N}=\frac{\nu p+(1+\nu) / 3}{p+2(1+\nu) / 3}
$$

respectively. This leads to the Laplace transformation $f_{v e}^{*}(p)$ of the solution of the linear viscoelastic problem. Here, the superscript $*$ is used as a short form for $\mathcal{L}$.

3. If necessary, prepare for the inverse transformation by expansion of $f_{v e}^{*}(p)$ into partial fractions.

4. Achieve the viscoelastic solution $f_{v e}(v)$ by the inverse Laplace transformation $f_{v e}(v)=\mathcal{L}^{-1}\left[f_{v e}^{*}(p)\right]$. The inverse Laplace transformation is found by use of corresponding tables ([118, Tab. 7.1] and [108, p. 1118-1123]).

\subsubsection{The viscoelastic Stoney equation}

\section{Elastic film with constant thickness}

The most simple equation for calculation of the curvature $k$ of a substrate with thickness $t_{s}$, Young's modulus $E_{s}$ and Poisson's number $\nu_{s}$ coated by a continuous film of thickness $t_{f}$ and film stress $\sigma$ is the Stoney equation [11

$$
k=\frac{6\left(1-\nu_{s}\right)}{E_{s}} \frac{t_{f}}{t_{s}^{2}} \sigma .
$$

By Laplace transformation and replacement of $E_{s}$ and $\nu_{s}$, the Laplace transformation of the viscoelastic version is obtained:

$$
k_{v e}^{*}=\frac{6(1-\mathcal{N})}{\mathcal{M}} \frac{t_{f}}{t_{s}^{2}} \sigma / p
$$

Inserting Eqs. (4.2) and (4.3) (with $E \rightarrow E_{s}$ and $\nu \rightarrow \nu_{s}$ ) for $\mathcal{M}$ and $\mathcal{N}$, respectively, and expansion into partial fractions leads to

$$
k_{v e}^{*}=\frac{6 t_{f}}{E_{s} t_{s}^{2}} \sigma\left[\frac{1-\nu_{s}}{p}+\frac{1+\nu_{s}}{3 p^{2}}\right] .
$$

The inverse Laplace transformation in combination with $v=t / \tau_{s}$ results in the viscoelastic version of the Stoney equation

$$
k_{v e}(t)=\frac{6 t_{f}}{E_{s} t_{s}^{2}} \sigma\left[1-\nu_{s}+\frac{1+\nu_{s}}{3} \frac{t}{\tau_{s}}\right] .
$$

Eq. (4.7) is plotted in Fig. 4.1. A linear dependence of the curvature on time is observed. The slope increases with increasing film stress $\sigma$ and decreases with increasing relaxation time $\tau_{s}$. Please note that for $t=0$ the elastic Eq. (4.4) is necessarily obtained. 


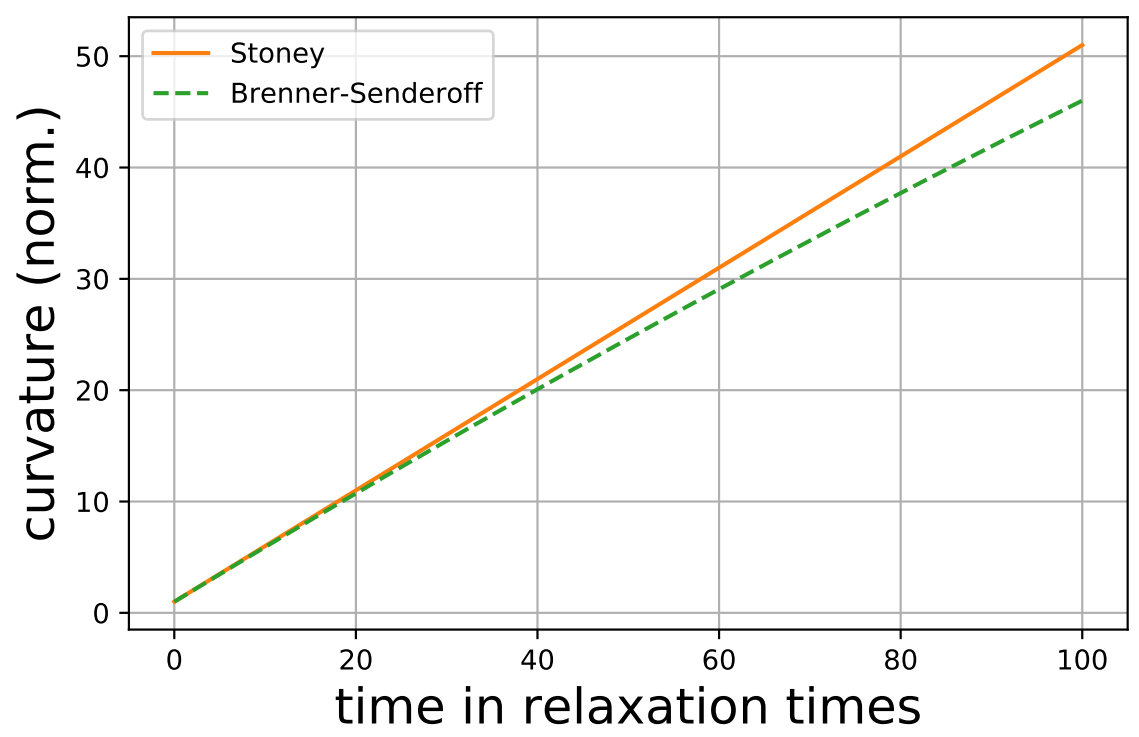

Figure 4.1: Comparison of the calculated viscoelastic versions of the Stoney equation (4.7) and the equation of Brenner and Senderoff (4.35). The time $t$ is given in units of the relaxation time $\tau_{s}$ and the curvature is normalized by the elastic curvature $k_{v e}(t=0)$ calculated by the Stoney Eq. (4.4). A film thickness of $t_{f}=1 \cdot 10^{-3} t_{s}$ and elastic properties of $E_{f}=E_{s}$ and $\nu_{s}=\nu_{f}=0.2$ have been assumed.

\section{Parabolic film growth with exponential stress decay}

Related to the experimental part of this thesis, we now seek an analytic description of the curvature evolution for the case that the film stress is generated by a parabolic oxidation process (cf. [2]) and that the stress in the oxide layer relaxes exponentially with time. First, the elastic case is considered. For this, the integrated stress

$$
N=\int_{0}^{t_{f}} \sigma_{f}^{\prime}(x) \mathrm{d} x
$$

is introduced with the stress distribution $\sigma_{f}^{\prime}(x)$ inside the oxide layer. We now assume a parabolic rate law for the film thickness $x$ in dependence on time $s$ :

$$
x=\sqrt{B_{\mathrm{dg}} s} \Rightarrow \mathrm{d} x=\frac{1}{2} \sqrt{\frac{B_{\mathrm{dg}}}{s}} \mathrm{~d} s .
$$

Inserting Eq. (4.9) into Eq. 4.8 the equation

$$
N(t)=\frac{\sqrt{B_{\mathrm{dg}}}}{2} \int_{0}^{t} \frac{\sigma_{f}(s)}{\sqrt{s}} \mathrm{~d} s
$$

is obtained for the integrated stress in dependence on time $t$ and for a film stress $\sigma_{f}(s)$. It is now assumed that the film stress decays exponentially with the relaxation time $\tau_{f}$ after creation at time $s$. Thus,

$$
\sigma_{f}(s)=\sigma_{0} \exp \left[-(t-s) / \tau_{f}\right]
$$


By insertion into Eq. 4.10) and substitution of $\sigma \cdot t_{f}$ in the Stoney Eq. (4.4) by $N(t)$, an extended version of the elastic Stoney equation

$$
k(t)=\frac{3\left(1-\nu_{s}\right) \sqrt{B_{\mathrm{dg}}} \sigma_{0}}{E_{s} t_{s}^{2}} \int_{0}^{t} \frac{\exp \left[-(t-s) / \tau_{f}\right]}{\sqrt{s}} \mathrm{~d} s
$$

is obtained. Please note that Eq. 4.12) and its derivation are related to the calculations made in [148], [149] and in [81] for the oxidation of a silicon or a metal surface, respectively.

For calculation of the viscoelastic version of Eq. (4.12), the substitution $t=\tau_{s} \cdot v$ is made, the Laplace transformation is calculated, and $E_{s}$ and $\nu_{s}$ are substituted by $\mathcal{M}$ and $\mathcal{N}$, respectively:

$$
k_{v e}^{*}=a \frac{1-\mathcal{N}}{\mathcal{M}} \mathcal{L}[I(v)]
$$

Here, the abbreviations

$$
a=\frac{3 \sqrt{B_{\mathrm{dg}}} \sigma_{0}}{t_{s}^{2}}
$$

and

$$
I(v)=\int_{0}^{v \cdot \tau_{s}} \frac{\exp \left[-(t-s) / \tau_{f}\right]}{\sqrt{s}} \mathrm{~d} s
$$

have been introduced. By insertion of Eqs. 4.2 and 4.3 for $\mathcal{M}$ and $\mathcal{N}$, respectively, the equation

$$
k_{v e}^{*}=\frac{a\left(1-\nu_{s}\right)}{E_{s}} \mathcal{L}[I(v)]+\frac{a\left(1+\nu_{s}\right)}{3 E_{s} p} \mathcal{L}[I(v)]
$$

is obtained. The inverse Laplace transformation of the first term can be readily obtained. However, for the inverse Laplace transformation of the second term, the Laplace transform $\mathcal{L}[I(v)]$ has to be evaluated. In section $\mathrm{B}$ in the appendix, it is demonstrated that

$$
\mathcal{L}[I(v)]=\sqrt{\pi \cdot \tau_{s}} \frac{1}{\sqrt{p}\left(p+\tau_{s} / \tau_{f}\right)} .
$$

Insertion into Eq. 4.16) and expansion into partial fractions leads to

$$
k_{v e}^{*}=\frac{a\left(1-\nu_{s}\right)}{E_{s}} \mathcal{L}[I(v)]+\frac{a\left(1+\nu_{s}\right) \tau_{f} \sqrt{\pi}}{3 E_{s} \sqrt{\tau_{s}}}\left[\frac{1}{p^{3 / 2}}-\frac{1}{\sqrt{p}\left(p+\tau_{s} / \tau_{f}\right)}\right] .
$$

By use of Eq. (4.17), the inverse Laplace transformation of this equation is calculated, and by insertion of Eq. (4.15) and Eq. (4.14), the final expression for the linear viscoelastic version of the extended Stoney Eq. (4.12) is obtained:

$$
\begin{aligned}
k_{v e}(t) & =\frac{3 \sigma_{0} \sqrt{B_{\mathrm{dg}}}}{E_{s} t_{s}^{2}}\left[\left(1-\nu_{s}\right) \int_{0}^{t} \frac{\exp \left[-(t-s) / \tau_{f}\right]}{\sqrt{s}} \mathrm{~d} s\right. \\
& \left.+\frac{\left(1+\nu_{s}\right) \tau_{f}}{3 \tau_{s}}(\underbrace{2 \sqrt{t}}_{\text {square root term }}-\underbrace{\int_{0}^{t} \frac{\exp \left[-(t-s) / \tau_{f}\right]}{\sqrt{s}} \mathrm{~d} s}_{\text {integral term }})\right] .
\end{aligned}
$$


In Fig. 4.2, the integral term

$$
\int_{0}^{t} \frac{\exp \left[-(t-s) / \tau_{f}\right]}{\sqrt{s}} \mathrm{~d} s
$$

and the square root term $2 \sqrt{t}$ of Eq. 4.19 are plotted in dependence on the time $t$ measured in relaxation times $\tau_{f}$. For large times $t \gg \tau_{f}$ the integral term and the elastic deformation can be neglected, and the curvature evolution is described by

$$
\begin{aligned}
k_{v e}(t) & \approx \frac{2\left(1+\nu_{s}\right) \sigma_{0}}{E_{s} t_{s}^{2}} \frac{\tau_{f}}{\tau_{s}} \sqrt{B_{\mathrm{dg}} \cdot t} \\
& =\frac{2\left(1+\nu_{s}\right) \sigma_{0}}{E_{s} t_{s}^{2}} \frac{\tau_{f}}{\tau_{s}} x(t) .
\end{aligned}
$$

Thus, by comparison with Eq. (4.9) for the oxide thickness, it can be seen that the curvature $k_{v e}(t)$ is proportional to the oxide thickness $x(t)=\sqrt{B_{\mathrm{dg}} \cdot t}$. The intuitive explanation of the square-root behavior in Eq. (4.21) is that the rate of curvature change is determined by an effective film thickness that is limited by the stress relaxation in the film and depends on the current oxidation rate. However, due to the parabolic oxidation process, the oxidation rate decreases with on going oxidation. Thus the effective film thickness decreases and consequently the curvature evolution flattens with time. Only at small oxidation times $t \lesssim \tau_{f}$, the effective film thickness is not limited by the stress relaxation but by the oxidation process. This explains the substraction of the integral term 4.20 in Eq. 4.19): It corrects for the fact that the film thickness starts at zero, and therefore, the viscous flow rate has to be zero at $t=0$.

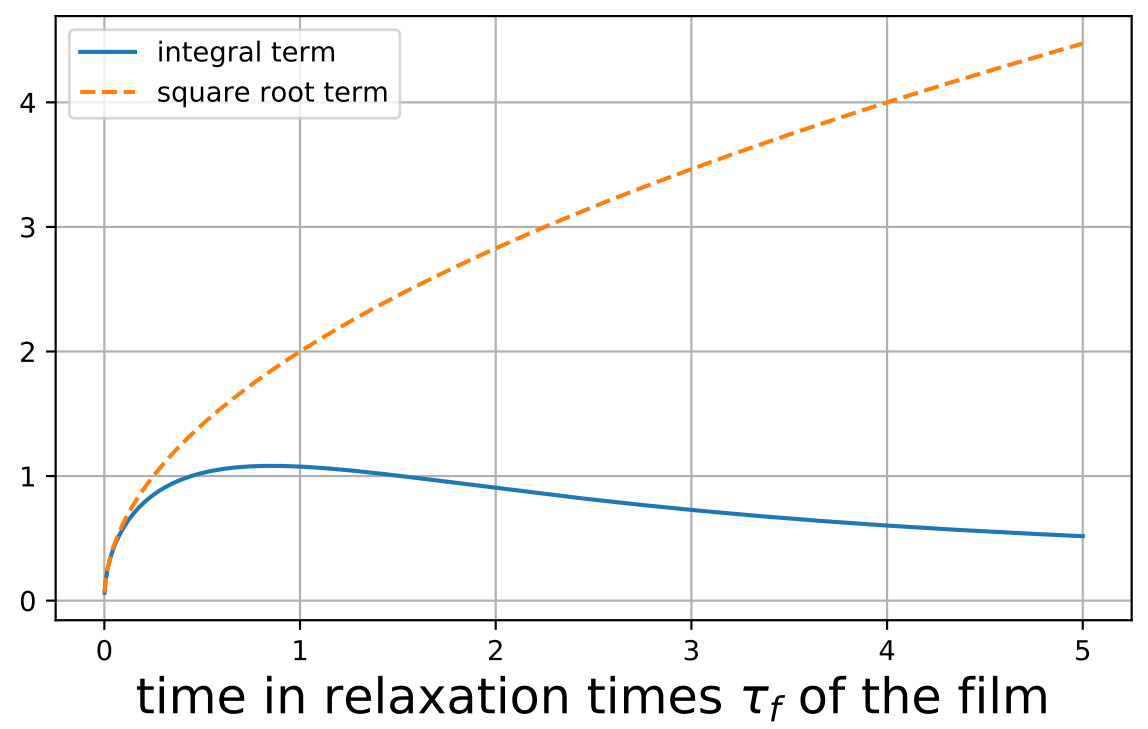

Figure 4.2: Comparison of the integral term and the square root term of the derived Eq. 4.19) for curvature evolution during parabolic oxidation. The terms are plotted in dependence on the time $t$ in units of the relaxation time $\tau_{f}$ of the film.

An estimate obtained by Eq. 4.19) for the curvature evolution of a real sample, as studied in this thesis, is plotted in Fig. 4.3a. A fused silica substrate of $1 \mathrm{~mm}$ 
thickness covered with a thin film of $\mathrm{SiO}_{x}$ is considered. During annealing at $1120^{\circ} \mathrm{C}$, the film shall oxidize by a diffusion limited process. If the stoichiometry of the $\mathrm{SiO}_{x}$ film is close to the one of $\mathrm{SiO}$, the same oxide thickness as in oxidation of a silicon surface is reached by comsumption of only half the number of oxygen atoms. Thus, it is assumed that the instantaneous growth stress $\sigma_{0}$ is half the value of $\sigma_{\mathrm{Si}}=460(50) \mathrm{MPa}[150]$ for oxidation of a silicon surface, and the parabolic rate constant $B_{\mathrm{dg}}$ is double the value $B_{\mathrm{dg}, \mathrm{Si}}=0.027 \mu \mathrm{m}^{2} / \mathrm{h}$ [2] for dry oxidation of a silicon surface at $1100^{\circ} \mathrm{C}$. In 151,152 , the relationship

$$
\frac{\mathrm{d} \sigma_{f}}{\mathrm{~d} t}=-\frac{E_{f}}{1-\nu_{f}} \frac{\sigma_{f}}{6 \eta_{f}}
$$

is given for the change of the film stress $\sigma_{f}$ in dependence on Young's modulus $E_{f}$, Poisson's number $\nu_{f}$ of the film and material shear viscosity $\eta_{f}$. By insertion of Eq. (4.11) for the exponential relaxation of the film stress with $s=0$ into Eq. 4.23), and substitution of $E_{f}=2 G_{0}^{f}\left(1+\nu_{f}\right)$, the relationship

$$
\tau_{f}=3 \frac{1-\nu_{f}}{1+\nu_{f}} \eta_{f} / G_{0}^{f}
$$

for the relaxation time $\tau_{f}$ of the film is obtained. If for the film the mechanical properties of water-free fused silica are assumed, a value of $\eta_{f} \approx 1.6 \cdot 10^{13} \mathrm{~Pa} \cdot \mathrm{s}$ is obtained via Eq. (12) from [100, p. 14], and therefore $\tau_{f} \approx 0.5 \mathrm{~h}$ is estimated. For the substrate, I assumed a viscosity of $\eta_{s}=10^{12} \mathrm{~Pa} \cdot \mathrm{s}$, as given by the manufacturer for Heraeus Suprasil at $1120^{\circ} \mathrm{C}$. The substrate viscosity resulted in a substrate relaxation time of $\tau_{s} \approx 32 \mathrm{~s}$. Fig. $4.3 \mathrm{a}$. shows that a complex behavior of the curvature evolution during oxidation of the first few $100 \mathrm{~nm}$ is obtained by Eq. 4.19.

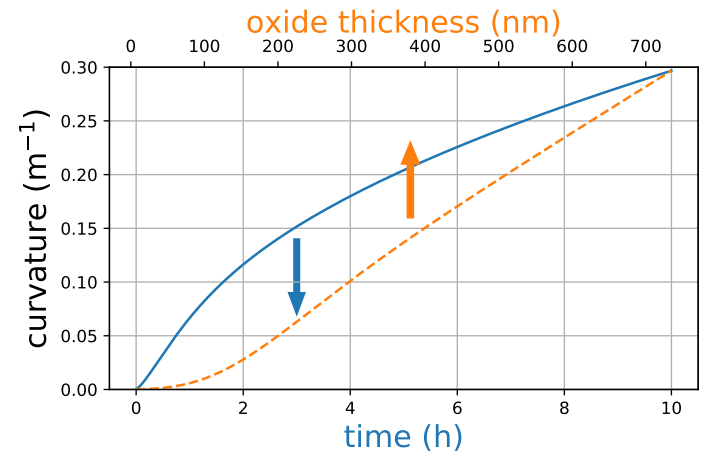

(a)

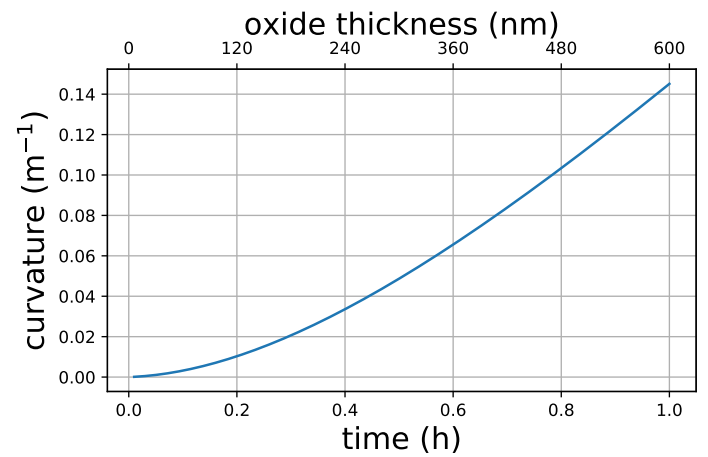

(b)

Figure 4.3: An estimate for the curvature evolution of a $1 \mathrm{~mm}$ thick fused silica plate covered by a film of $\mathrm{SiO}_{x}$ that is oxidized at $1120^{\circ} \mathrm{C}$ by (a) a diffusion controlled process and (b) a linear process.

\section{Linear film growth with exponential stress decay}

In the calculation above, we assumed a parabolic growth law for the film thickness. However, from the Deal-Grove model [2] it is known that the oxide thickness grows 
linearly with time for small oxidation times. Now, this kind of a linear growth law

$$
x=C \cdot s,
$$

with film thickness $x$, time $s$ and linear rate constant $C$, is considered. Insertion of Eq. 4.25 and Eq. 4.11) for the exponential relaxation of the film stress into Eq. (4.8) for the integrated stress leads to the expression

$$
N(t)=C \cdot \sigma_{0} \cdot \tau_{f}\left[1-\exp \left(-\frac{t}{\tau_{f}}\right)\right]
$$

for the integrated stress of the film after time $t$. By substitution of $N=\sigma \cdot t_{f}$ in the Stoney Eq. (4.4), the extended elastic version of the Stoney equation

$$
k(t)=\frac{6\left(1-\nu_{s}\right) C \sigma_{0} \tau_{f}}{E_{s} t_{s}^{2}}\left[1-\exp \left(-\frac{t}{\tau_{f}}\right)\right]
$$

is achieved.

A similar calculation as in the above two cases leads straight forward to the viscoelastic version of this equation:

$k_{v e}(t)=\frac{6 C \sigma_{0} \tau_{f}}{t_{s}^{2}}\left(\frac{1-\nu_{s}}{E_{s}}\left[1-\exp \left(-\frac{t}{\tau_{f}}\right)\right]+\frac{1+\nu_{s}}{3 E_{s} \tau_{s}}\left[t-\tau_{f}\left(1-\exp \left(-\frac{t}{\tau_{f}}\right)\right)\right]\right)$.

For $t>>\tau_{f}$, the curvature grows linearly with time $t$. Thus, also in this case the curvature $k_{v e}$ is proportional to the oxide thickness $t_{f}=C \cdot t$. Further, if the elastic deformation is neglected, the same Eq. (4.22) as for the parabolic growth law is obtained. Therefore, this can be understood as above: At large oxidation times the effective stressed film thickness is $C \cdot \tau_{f}$. Only at small oxidation times the effective stressed film is still growing.

As in the preceding section, Eq. (4.28) has been plotted for the case of a $1 \mathrm{~mm}$ thick fused silica plate covered by a thin $\mathrm{SiO}_{x}$ film (Fig. 4.3b). For the linear rate constant $C=B_{\mathrm{dg}} / A_{\mathrm{dg}}$, the same value for $B_{\mathrm{dg}}$ as above and $A_{\mathrm{dg}}=0.09 \mu \mathrm{m}[2]$ were assumed. A comparison of Figs. $4.3 \mathrm{a}$ and $4.3 \mathrm{~b}$ shows that the curvature is of the same order of magnitude at similar oxide thicknesses.

\subsubsection{The viscoelastic version of the equation of Brenner and Senderoff}

If the assumption $t_{r}=t_{f} / t_{s}<<1$ is not valid, the Stoney Eq. (4.4) loses its justification and the ratio

$$
E_{r}=\frac{B_{f}}{B_{s}}
$$

of the biaxial moduli $B_{s}=E_{s} /\left(1-\nu_{s}\right)$ and $B_{f}=E_{f} /\left(1-\nu_{f}\right)$ of substrate and film, respectively, has to be considered. This is achieved by Eq. (33) in [12] of Brenner and 
Senderoff. They derived this equation for a strip like (length $>>$ width) substrate. In [13], a version for a plate like (length $\approx$ width) substrate has been formulated and expanded to first order in $E_{r} t_{r}$ and $t_{r}$ :

$$
k=\frac{6\left(1-\nu_{s}\right)}{E_{s}} \frac{t_{f}}{t_{s}^{2}} \sigma_{f} \frac{1}{1+4 E_{r} t_{r}-t_{r}} .
$$

For calculation of the viscoelastic solution $k_{v e}$, only the term

$$
\frac{1-\nu_{s}}{E_{s}\left(1+4 E_{r} t_{r}-t_{r}\right)}=\frac{1}{1-t_{r}} \underbrace{\frac{1}{E_{s} /\left(1-\nu_{s}\right)+a^{\prime}}}_{=a}
$$

with $a^{\prime}=4 B_{f} t_{r} /\left(1-t_{r}\right)$ has to be considered. By a Laplace transformation of $a$ and replacement of $E_{s}$ and $\nu_{s}$ by $\mathcal{M}$ and $\mathcal{N}$, respectively, the equation

$$
a_{v e}^{*}=\frac{1}{p} \frac{1}{\mathcal{M} /(1-\mathcal{N})+a^{\prime}}
$$

is achieved. Insertion of Eqs. (4.2) and (4.3) (with $E \rightarrow E_{s}$ and $\nu \rightarrow \nu_{s}$ ), for $\mathcal{M}$ and $\mathcal{N}$, respectively, and expansion into partial fractions leads to the equation

$$
a_{v e}^{*}=\frac{1}{a^{\prime}}\left[\frac{1}{p}-\frac{1}{1+a^{\prime}\left(1-\nu_{s}\right) / E_{s}} \frac{1}{p+a^{\prime}\left(1+\nu_{s}\right) /\left(3\left(E_{s}+a^{\prime}\left(1-\nu_{s}\right)\right)\right)}\right] .
$$

Calculation of the inverse Laplace transformation and simplification gives

$$
a_{v e}(t)=\frac{1-\nu_{s}}{E+a^{\prime}\left(1-\nu_{s}\right)}+\frac{1}{a^{\prime}} \frac{1}{1+a^{\prime}\left(1-\nu_{s}\right) / E_{s}}\left[1-\exp \left(-\frac{1}{3} \frac{a^{\prime}\left(1+\nu_{s}\right)}{E_{s}+a^{\prime}\left(1-\nu_{s}\right)} \frac{t}{\tau}\right)\right] .
$$

This equation has to be reinserted into 4.31) and the result into (4.30) to finally achieve the viscoelastic version of the Brenner-Senderoff equation:

$$
\begin{aligned}
k_{v e}(t) & =\frac{6 \sigma_{f} t_{f}}{t_{s}^{2}} \frac{1}{1+4 E_{r} t_{r}-t_{r}}\left[\frac{1-\nu_{s}}{E_{s}}\right. \\
& \left.+\frac{1}{4} \frac{1-\nu_{f}}{E_{f}}\left(\frac{1}{t_{r}}-1\right)\left[1-\exp \left(-\frac{4}{3} \frac{1+\nu_{s}}{1-\nu_{s}} \frac{E_{r} t_{r}}{1+4 E_{r} t_{r}-t_{r}} \frac{t}{\tau}\right)\right]\right] .
\end{aligned}
$$

Eq. 4.35 is plotted in Fig. 4.1. If the absolute value of the argument of the exponential function is small, the function can be approximated by the zeroth and first element $(\exp (x) \approx 1+x)$ of its series expansion:

$$
k_{v e}(t) \approx \frac{6 \sigma_{f} t_{f}}{E_{s} t_{s}^{2}} \frac{1}{1+4 E_{r} t_{r}-t_{r}}\left[1-\nu_{s}+\frac{1}{3}\left(1-t_{r}\right) \frac{1+\nu_{s}}{1+4 E_{r} t_{r}-t_{r}} \frac{t}{\tau}\right]
$$

If $t_{r}=0$ is assumed, the viscoelastic Stoney Eq. (4.7) is obtained from the above equation. However, in the case of large times $t$, the absolute value of the argument of the exponential function is large and the curvature approaches

$$
k_{\lim }=\frac{3 \sigma_{f}\left(1-\nu_{f}\right)}{2 t_{s} E_{f}} .
$$


Although the Brenner-Senderoff Eq. 4.30 loses its validity for large deflections of the substrate [1, 13, 15, 16], Eq. 4.37) is a hint to limitations of the viscous deformation by a stressed film and gives an estimate for the dependencies of the asymptotic curvature. According to Eq. (4.37), the asymptotic curvature only depends on the substrate thickness $t_{s}$, the mechanical properties of the film and the film stress $\sigma_{f}$.

A comparison of the derived viscoelastic Stoney 4.7) and Brenner-Senderoff (4.35) equations with the result of a linear viscoelastic finite element simulation is shown in Fig. 4.4. A substrate of $1 \mathrm{~mm}$ thickness with a diameter of $25 \mathrm{~mm}$ is assumed. The substrate is coated by a continuous film of $1 \mu \mathrm{m}$ thickness and an initial homogeneous, compressive film stress of $-100 \mathrm{MPa}$. The mechanical behavior of the film is linear elastic while the substrate behaves linear viscoelastic. For both, film and substrate, the elastic properties of fused silica at room temperature (see Tab. A.1) and for the substrate a viscosity of $10^{12} \mathrm{~Pa} \cdot \mathrm{s}$ were set. The time increment of the simulation was $100 \mu \mathrm{h}$. As in the experiments, the curvature of the non coated sample side is observed in the simulation. Thus, a compressive film stress corresponds to a positive curvature. It can be seen that the simulation results are well described by the viscoelastic Brenner-Senderoff Eq. (4.35). The deviation is below $0.13 \%$ after $1 \mathrm{~h}$. On the opposite, the deviation of the simulation from the viscoelastic Stoney Eq. (4.7) is much larger with about $10 \%$ after $1 \mathrm{~h}$. This result gives confidence in the derived viscoelastic Brenner-Senderoff Eq. (4.35) and in the results of the simulation.

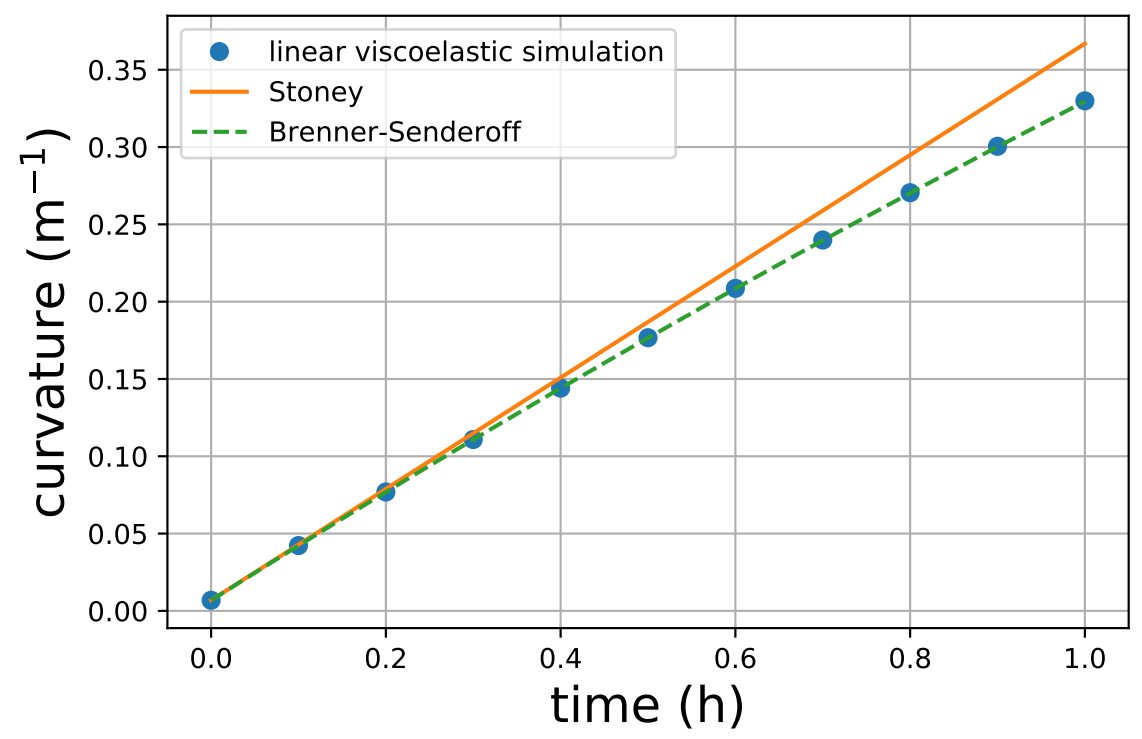

Figure 4.4: A comparison of the results of a linear viscoelastic finite element simulation, the viscoelastic Stoney Eq. (4.7) and the viscoelastic Brenner-Senderoff Eq. 4.35). A $1 \mu \mathrm{m}$ thick film with a stress of $-100 \mathrm{MPa}$ on a $1 \mathrm{~mm}$ thick fused silica substrate with a viscosity of $\eta=10^{12} \mathrm{~Pa} \cdot \mathrm{s}$ was assumed. The curvature of the non-coated side of the substrate is considered.

For understanding of the viscoelastic Brenner-Senderoff Eq. 4.35), Fig. 4.5 shows the film stress calculated by the above mentioned simulation. The absolute value of the film stress decreases by about $20 \%$ during $1 \mathrm{~h}$ of deformation. This shows 
that the decreasing rate of deformation, predicted by Eq. (4.35), is caused by the elastic relaxation of the film stress due to the deformation of the underlying substrate. Thus, Eq. 4.35 will fail if the film stress is generated not before, but during the deformation. The latter would be the case if stress generating processes, like oxidation, happen at the same time as viscous flow.

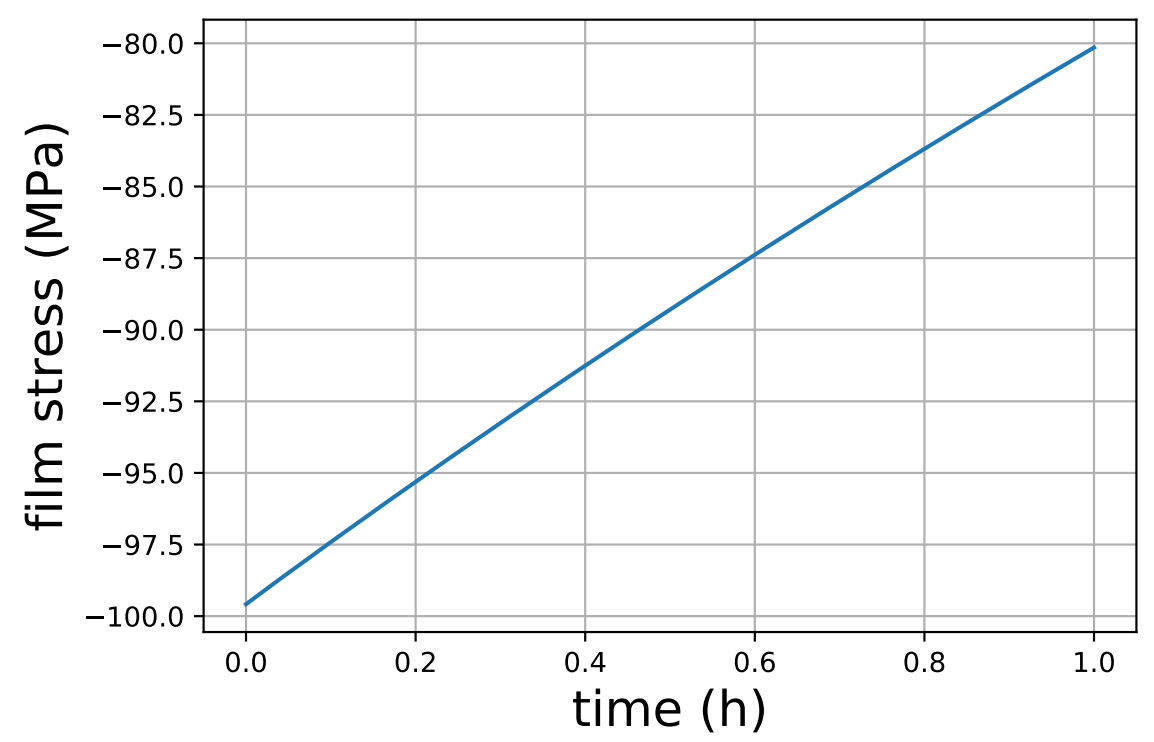

Figure 4.5: The time evolution of the film stress in the simulation of Fig. 4.4 .

\subsubsection{The viscoelastic version of the equation of Wikström et al.}

In [90], experimental data for the viscous deformation of fused silica plates during oxidation of a deposited $\mathrm{SiO}_{x}$ film is mentioned, that suggests that a line structured film leads to a highly anisotropic deformation. Here, an analytic approach is made to explain this behavior.

By Wikström et al. an extension of the Stoney Eq. (4.4) was derived for the case of a line structured film and thermal stresses [3] (Fig. 1.3a). For the elastic substrate, the theory gives the curvatures $k_{p}$ and $k_{s}$ along and across the lines, respectively, by

$$
\begin{aligned}
& k_{p}=\frac{6 t_{f} \sigma_{f}}{t_{s}^{2} E_{s}} \frac{b}{d}\left[1-\chi \nu_{f}-(1-\chi) \nu_{s}\right], \\
& k_{s}=\frac{6 t_{f} \sigma_{f}}{t_{s}^{2} E_{s}} \frac{b}{d}\left[1-\chi-\left(1-\chi \nu_{f}\right) \nu_{s}\right] .
\end{aligned}
$$

In Eqs. (4.38) and (4.39), the factor $b / d$ denotes the duty cycle of the line structure, calculated from linewidth $b$ and line period $d$, and quantity $\chi=\chi(\rho)$ is a function of the aspect ratio $\rho=t_{f} / b$ of the lines. $\chi(\rho)$ is an element of the intervall $[0,1)$ with $\chi(\rho \rightarrow 0)=0$ and $\chi(\rho \rightarrow \infty)=1[3]$. 
For calculation of Versions of Eqs. (4.38) and $(4.39)$ for the case of a viscoelastic substrate and an elastic film, it is assumed that the geometry of the line structure, the properties of the film material and the quantity $\chi$ are independent of time. However, the presumably most critical assumption is that $\chi$ is the same in the viscoelastic as in the elastic case. This is because the calculation of $\chi$ is based on stress intensity factors for a homogeneous medium 21 and therefore does not account for different material properties of film and substrate [3]. Nevertheless, it is expected that even with this weakly justified assumption something about the viscoelastic deformation in case of a line structured film can be learned.

Under the above assumptions, the Laplace transform of the viscoelastic version of Eq. (4.38) is given by

$$
\left(k_{p}^{v e}\right)^{*}=a \frac{1}{p \mathcal{M}}\left[1-\chi \nu_{f}-(1-\chi) \mathcal{N}\right]
$$

with $a=6 t_{f} \sigma_{f} b /\left(t s^{2} d\right)$. By insertion of Eqs. 4.2) and (4.3) (with $E \rightarrow E_{s}$ and $\left.\nu \rightarrow \nu_{s}\right)$, for $\mathcal{M}$ and $\mathcal{N}$ and expansion into partial fractions, the equation

$$
\left(k_{p}^{v e}\right)^{*}=\frac{a}{E_{s}}\left[\left(1-\chi \nu_{f}\right)\left(\frac{1}{p}+\frac{2\left(1+\nu_{s}\right)}{3 p^{2}}\right)-(1-\chi)\left(\frac{\nu_{s}}{p}+\frac{1+\nu_{s}}{3 p^{2}}\right)\right]
$$

is obtained. From the inverse Laplace transformation, it follows that

$$
k_{p}^{v e}(t)=\frac{6 t_{f} \sigma_{f} b}{E_{s} t_{s}^{2} d}\left[1-\chi \nu_{f}-(1-\chi) \nu_{s}+\frac{1}{3}\left(1+\nu_{s}\right)\left(1+\chi-2 \chi \nu_{f}\right) \frac{t}{\tau_{s}}\right] .
$$

And analogously to the above derivation, the equation

$$
k_{s}^{v e}(t)=\frac{6 t_{f} \sigma_{f} b}{E_{s} t_{s}^{2} d}\left[1-\chi-\left(1-\chi \nu_{f}\right) \nu_{s}+\frac{1}{3}\left(1+\nu_{s}\right)\left(1-2 \chi+\chi \nu_{f}\right) \frac{t}{\tau_{s}}\right]
$$

for the curvature across the lines is obtained from Eq. (4.39).

In the derived Eqs. 4.42 and 4.43 the curvature changes linear with time. However, the rates of change are different for the direction along and across the lines. Therefore, for large times $t$, the curvature ratio

$$
k_{r}^{v e}=k_{s}^{v e} / k_{p}^{v e}=\frac{1-\chi-\left(1-\chi \nu_{f}\right) \nu_{s}+\frac{1}{3}\left(1+\nu_{s}\right)\left(1-2 \chi+\chi \nu_{f}\right) \frac{t}{\tau_{s}}}{1-\chi \nu_{f}-(1-\chi) \nu_{s}+\frac{1}{3}\left(1+\nu_{s}\right)\left(1+\chi-2 \chi \nu_{f}\right) \frac{t}{\tau_{s}}}
$$

will adopt a constant value given by the the ratio of the slopes:

$$
\lim _{t \rightarrow \infty} k_{r}^{v e}=\frac{1-\chi\left(2-\nu_{f}\right)}{1+\chi\left(1-2 \nu_{f}\right)}
$$

Please note that the final curvature ratio does only depend on $\chi$ and $\nu_{f}$.

In Fig. 4.6a, the curvature ratio $k_{r}^{v e}$ is plotted as a function of time $t$ in relaxation times $\tau_{s}$ for $\rho=0.1$ and $\nu_{f}=\nu_{s}=0.165$. Starting at the elastic solution (as it always has to be), the curvature ratio decreases with time and asymptotically reaches the limit given by Eq. 4.45). In Fig. 4.6b this final curvature value is 
plotted in dependence on the aspect ratio $\rho$ for the same Poisson's ratio as above. It is always smaller than the elastic solution. The difference is much more pronounced for large aspect ratios because the elastic solution tends to $k_{r}^{e}=-\nu_{s}=-0.165$ for $\chi \rightarrow 1[3]$, while the viscoelastic solution tends to $k_{r}^{v e}=-0.5$ independent of material parameters. In both cases, the curvature ratio changes its sign from positive for small aspect ratios to negative for large ones.

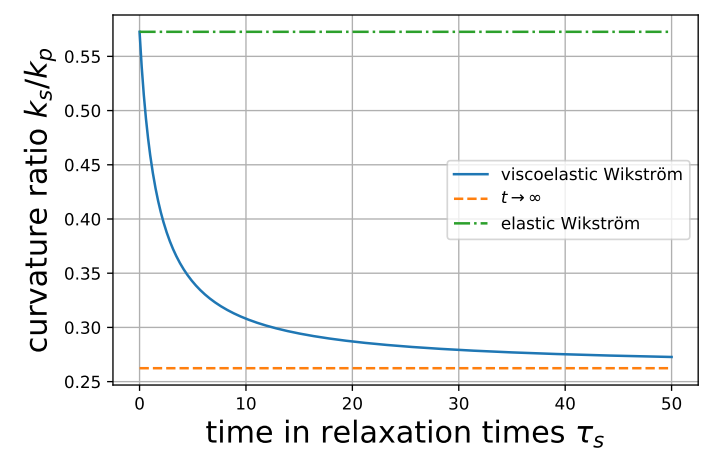

(a) $\rho=0.1$

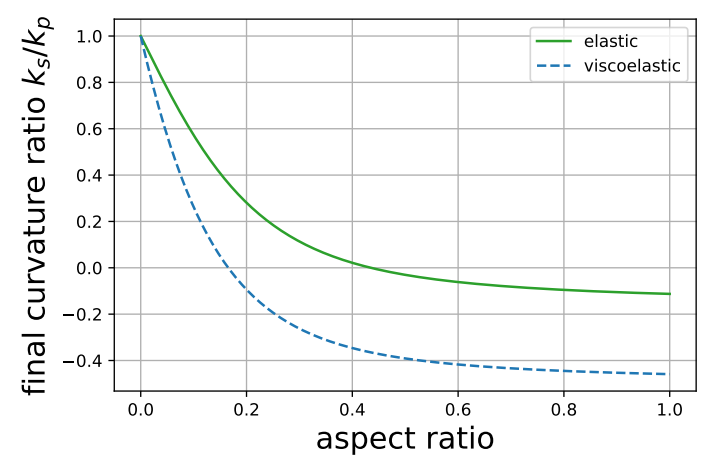

(b)

Figure 4.6: The curvature ratio as calculated by the elastic theory of Wikström et al. [3] and by its expansion to a simple viscoelastic behavior of the substrate (4.44). For the Poisson's ratios of substrate and film, $\nu_{s}=\nu_{f}=0.165$ was assumed. (a) The curvature ratio in dependence on time measured in units of the relaxation time $\tau_{s}$ for an aspect ratio of $\rho=0.1$. The elastic solution and the solution for large times are also denoted by horizontal lines. (b) The solution for large times in dependence on the aspect ratio $\rho$ compared to the elastic solution.

\subsection{Deformation by gravity}

If the viscosity of the substrate is low enough for observation of viscous flow due to a stressed film, a significant gravitational deformation of the substrate might also happen. That is why, the gravitational sag of linear viscoelastic plates is studied by the FEM in this section.

For the simulations, an initially flat fused silica substrate with a radius of $r=$ $12.5 \mathrm{~mm}$ and a thickness of $t_{s}=1 \mathrm{~mm}$ is assumed (Fig. 4.7). For the elastic properties $E_{s}$ and $\nu_{s}$, and the mass density $\rho_{m}$, the properties in Tab. A.1, in the appendix, at room temperature are used. The viscosity is set to $10^{12} \mathrm{~Pa} \cdot \mathrm{s}$, corresponding to a substrate made of Heraeus Suprasil and, according to the manufacturer, a temperature of $1120^{\circ} \mathrm{C}$. The gravitational force points in the $-z$ direction, while the plate is oriented in the $x-y$ plane with the center point of the upper surface in the origin. The mesh size is $0.5 \mathrm{~mm}$ and the time increment $100 \mu \mathrm{h}$. In the following, for better comparability with the experimental results, the deformation of the bottom surface is considered. Thus, a convex deformation of this surface causes a negative curvature' $\mathrm{2}^{2}$ (Fig. 4.8).

\footnotetext{
${ }^{2}$ To remember the sign of curvature for a convex or concave surface, think of a parabola $f(x)=$
} 


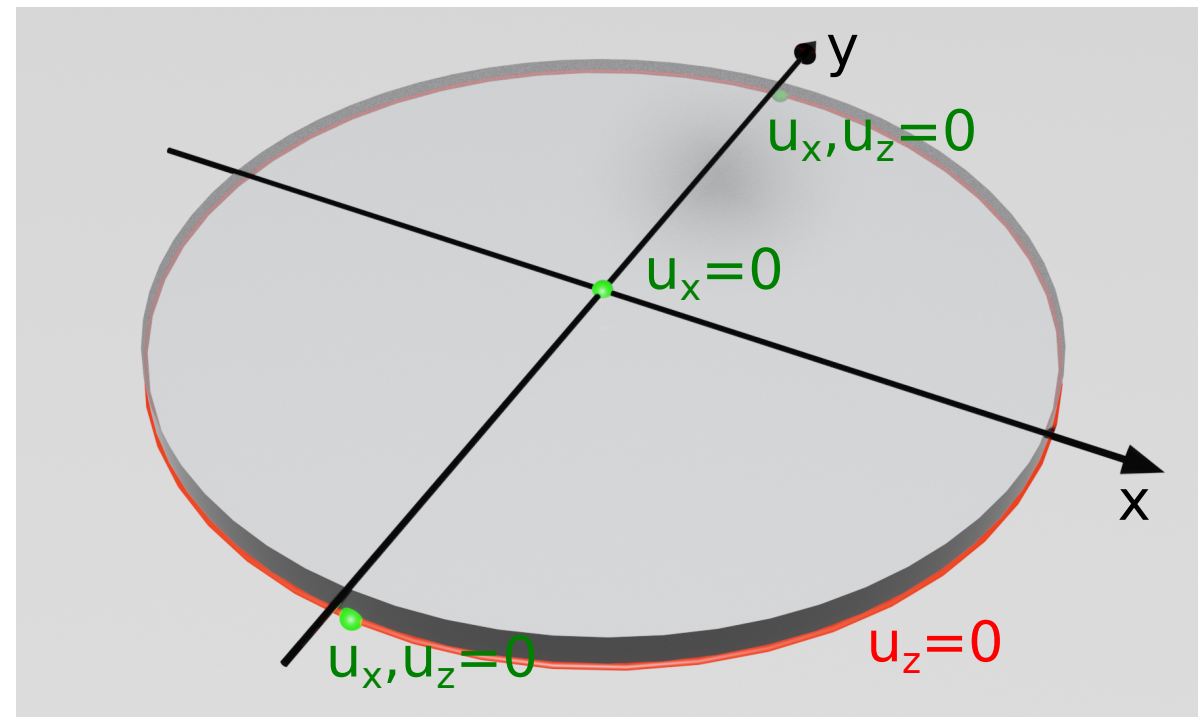

Figure 4.7: For the numerical study of the gravitational deformation of a viscoelastic plate, two different sets (red and green) of boundary conditions were applied.

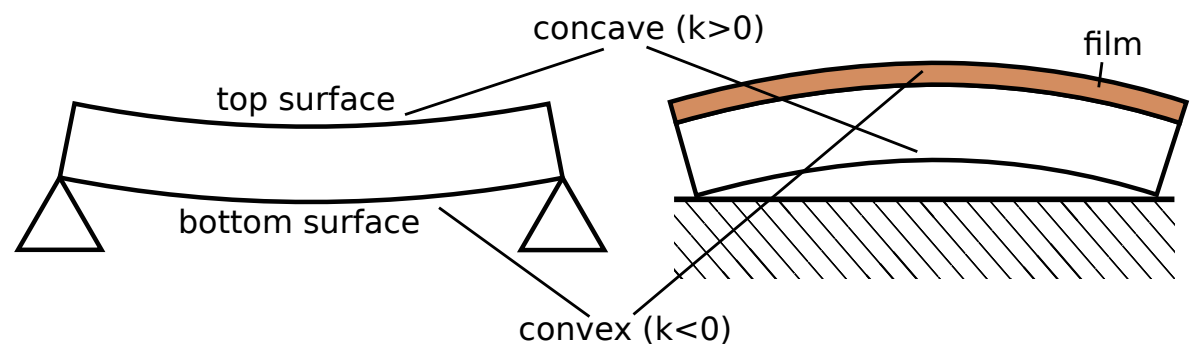

Figure 4.8: To avoid confusion, two different deformations of a plate are depicted. Left: The plate rests at the bottom rim. Therefore, gravity leads to a concave (positive curvature $k$ ) deformation of the top surface and to a convex deformation (negative curvature) of the bottom surface. Right: The plate was deformed by a compressively stressed thin film on its top surface. Thus, the top surface is convex and the bottom surface concave. 


\subsubsection{Bearing at the lower edge}

For the first case, it is assumed that the plate is supported at the lower edge of the rim (red in Fig. 4.7). Thus, the Dirichlet boundary condition for the displacement is $u_{z}(x, y, z)=0$ at $x^{2}+y^{2}=r^{2}$ and $z=-t_{s}$. This boundary condition imitates the case of a plate with a concave deformation of the bottom surface of isotropic curvature lying on a flat surface (Fig. 4.8). Fig. 4.9a shows three simulated curvature profiles of the bottom surface of an initially flat plate for the above boundary condition after $0,0.5$ and $1 \mathrm{~h}$ of deformation. The curvature is non-uniform with the minimum value in the middle of the plate. After $1 \mathrm{~h}$, the minimum curvature lies at about $-0.0055 \mathrm{~m}^{-1}$. The average curvature in the range of -7.5 up to $7.5 \mathrm{~mm}$ of the curvature profiles, calculated as in the experiments of Ch. 5 , is plotted in Fig. $4.9 \mathrm{~b}$ in dependence on the annealing time. A linear dependence is observed. After $1 \mathrm{~h}$ of deformation an average curvature of $-0.0046 \mathrm{~m}^{-1}$ is reached.

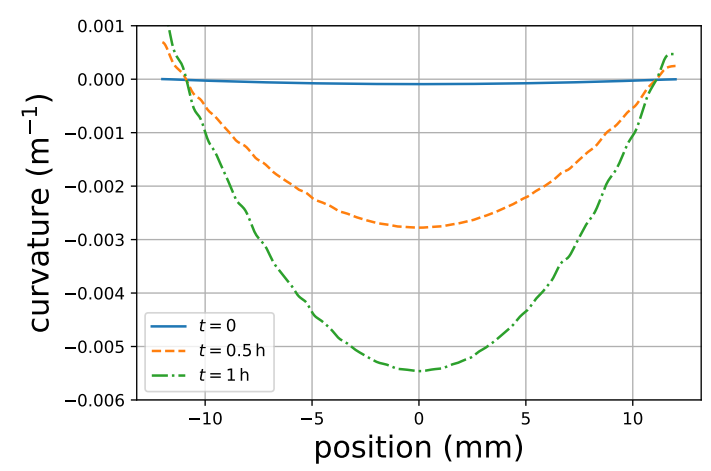

(a)

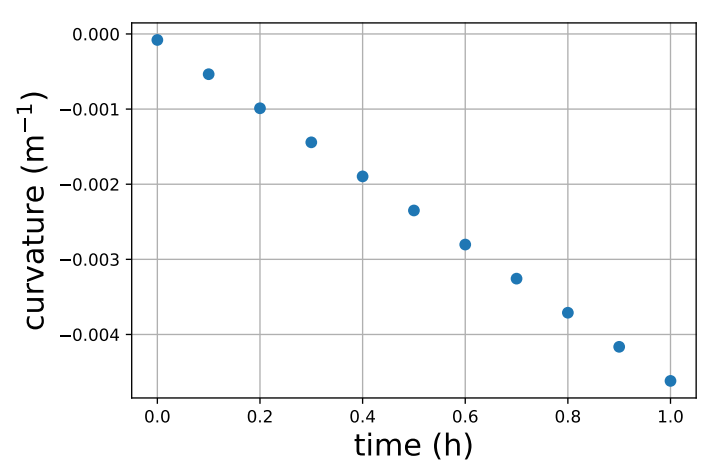

(b)

Figure 4.9: The curvature evolution of a fused silica plate with a thickness of $1 \mathrm{~mm}$ and a diameter of $25 \mathrm{~mm}$ during linear viscoelastic deformation by gravity. The plate is supported at the edge. (a) The curvature profiles after a specific deformation time. (b) The average curvature profiles in the middle $15 \mathrm{~mm}$ of the plate in dependence on deformation time. The time increment of the simulation was $\Delta t=100 \mu \mathrm{h}$. The curvature of the bottom surface is considered.

\subsubsection{Bearing at two opposite points}

I now assume that the sample is only supported at two opposite contact points lying on the $\mathrm{y}$ axis (green in Fig. 4.7). Thus, $u_{z}\left(0, \pm r,-t_{s}\right)=0$. Actually, this defines an unstable configuration even without deformations allowed. Therefore, rotation around the $\mathrm{y}$ axis is hindered by the additional boundary conditions of $u_{x}\left(0, \pm r,-t_{s}\right)=0$ and $u_{x}(0,0,0)=0$. The whole set of boundary conditions imitates the case of a plate on a flat surface with a concave deformation of the bottom surface, which is more pronounced in $\mathrm{y}$ than in $\mathrm{x}$ direction. Such a deformation can be caused by a line structured film with the line orientation along the y direction (cf. Sec. 5.1.4).

$a \cdot x^{2}$ and its second order coefficient $a$, which, according to Eq. 2.2., denotes half the curvature at $x=0$. If $a>0$, the parabola is concave. If $a<0$, the parabola is convex. 
Fig. 4.10a shows the resulting curvature profiles on the $\mathrm{x}$ and the $\mathrm{y}$ axis after $1 \mathrm{~h}$ of deformation. The curvature profile in $\mathrm{x}$ direction is clearly non-uniform and exhibits a maximum in the middle of the plate. In y direction a much more uniform curvature is observed in between -10 to $10 \mathrm{~mm}$. Fig. 4.10b shows the time evolution of the average curvatures in the range of -7.5 up to $7.5 \mathrm{~mm}$. In the y direction, the curvature decreases, while in the $\mathrm{x}$ direction the curvature increases. Please note that the decrease in average curvature in the y direction is about five times as fast than in the above case of support at the full edge.

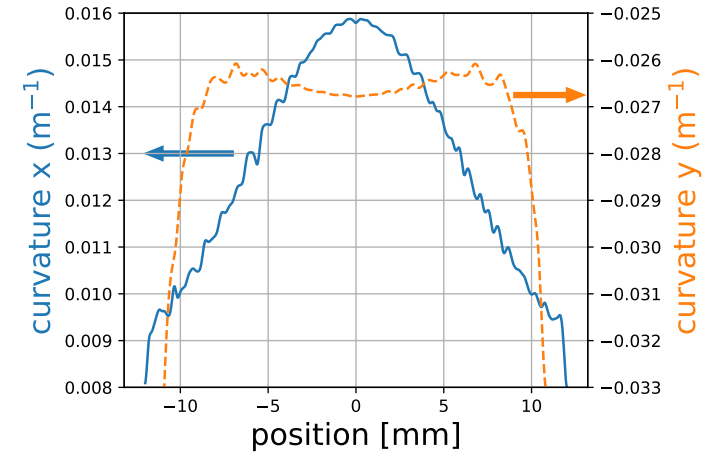

(a)

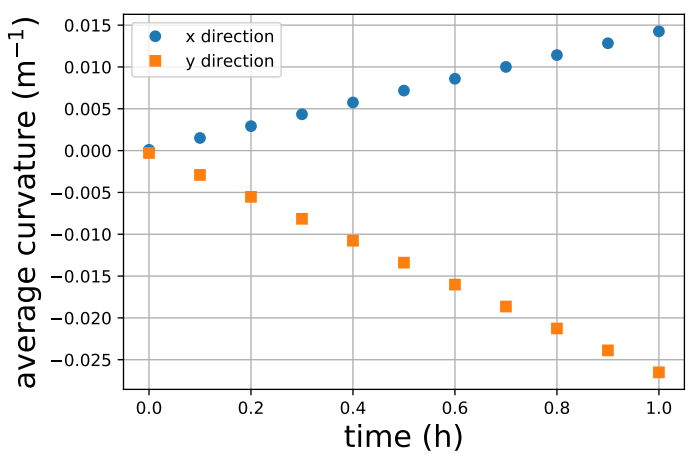

(b)

Figure 4.10: The curvature evolution of a fused silica plate with a thickness of $1 \mathrm{~mm}$ and a diameter of $25 \mathrm{~mm}$ during linear viscoelastic deformation by gravity. The plate is supported at two opposite points on the edge along the y axis. (a) The curvature profiles along the $\mathrm{x}$ and the $\mathrm{y}$ axis after $1 \mathrm{~h}$ of deformation. (b) The average curvature profiles in those directions in the middle $15 \mathrm{~mm}$ of the plate in dependence on deformation time. The time increment of the simulation was $\Delta t=100 \mu \mathrm{h}$. The curvature of the bottom surface is considered. 


\section{Chapter 5}

\section{Viscoelastic deformation of fused silica plates covered with a continuous or patterned silicon suboxide film}

It was found that oxidation in air of $\mathrm{SiO}_{x}$ films on fused silica substrates leads to a significant deformation of the samples and that this deformation depends on the patterning of the $\mathrm{SiO}_{x}$ film [90]. It was one of the tasks of this thesis to study this deformation in detail. For that, substrates of Heraeus Suprasil with a thickness of $1 \mathrm{~mm}$ and a diameter of $25 \mathrm{~mm}$ were coated with $\mathrm{SiO}_{x}$ films ${ }^{1}$. I structured the films by laser ablation and annealed the samples in oxidizing and almost inert atmospheres at such temperatures that a mostly viscous deformation would be expected. The results of these experiments are described in the following Sec. 5.1 .

I also pursued the idea to change the film stress of the $\mathrm{SiO}_{x}$ films via oxidation at temperatures so low that an entirely elastic deformation of the silica substrate is obtained. Although this approach was not successful in the end, it led to some interesting experimental results on the change of the $\mathrm{SiO}_{x}$ film during annealing, its oxidation kinetics, the oxidation stress and the stresses induced during front side and backside ablation of $\mathrm{SiO}_{x}$ film on a fused silica substrate. These results are presented in Sec. 5.2. The results of both sections are discussed in Sec. 5.3 .

\subsection{Results on the viscous deformation}

\subsubsection{Annealing in air at $1120^{\circ} \mathrm{C}$ in horizontal orientation}

In this section, results are shown for annealing in air and the samples lying on a ground fused silica plate with the coated side facing upwards (Fig. 2.9a). I chose the

\footnotetext{
${ }^{1}$ Deposition done by Lukas Richter (IFNano)
} 
annealing temperature to be $1120(1){ }^{\circ} \mathrm{C}$, which is the annealing point $\left(\eta=10^{12} \mathrm{~Pa} \cdot \mathrm{s}\right.$ [134, p. 112]) of the material, as stated by the manufacturer [97].

Fig. 5.1 summarizes the results for annealing of a sample with a 500(6) nm thick, continuous $\mathrm{SiO}_{x}$ film. In Fig. 5.1a transmission spectra of the sample after different total annealing times are shown. The transmission increases with increasing annealing time and eventually reaches the transmission of the substrate after $9 \mathrm{~h}$ of annealing. This behavior can be explained by the oxidation of the film to silicon dioxide 87, 153 158. By analysis of the reflection spectra of the coated sample side (not shown), I measured the thickness of the grown $\mathrm{SiO}_{2}$ layer on top of the $\mathrm{SiO}_{x}$ film (cf. Sec. 5.2.3). The results are given in the legend of Fig. 5.1a and are plotted in Fig. $5.1 \mathrm{~b}$ against the total annealing time. As expected, the thickness increases with increasing annealing time until after $9 \mathrm{~h}$ of annealing no interference peaks can be observed anymore. Thus, oxidation is complete. By fitting the $\mathrm{SiO}_{2}$ thickness values with a square root dependence $x_{\mathrm{SiO} 2}(t)=\sqrt{B_{\mathrm{dg}} \cdot t}$ (Fig. 5.1b), I obtained a parabolic rate constant of $B_{\mathrm{dg}}=0.04596(2) \mu \mathrm{m}^{2} / h$.

I observed the deformation of the sample by each time measuring two line profiles on the non-coated side (the lower side during annealing) of the sample in between the annealing steps, which are oriented as in Fig. 2.5b. Fig. 5.1c shows selected profiles in direction 1 . Initially, the substrate is virtually flat but develops a concave parabolic shape of the non-coated side during oxidation. The deflection increases with increasing annealing time until, after $9 \mathrm{~h}$, oxidation is complete. For longer annealing times, the deflection decreases again. A topography map of the non-coated side of the sample after $9 \mathrm{~h}$ of annealing is plotted in Fig. 5.1d. The deformation is isotropic. This observation is confirmed by comparison of the height profiles measured in direction 1 (Fig. 5.1c) to the height profiles measured in direction 2 (not shown).

From the measured height profiles, I calculated the curvature profiles, which are plotted in Fig. 5.1e. Up to $9 \mathrm{~h}$ of total annealing time, the curvature is roughly homogeneous along the measurement direction and increases with increasing annealing time. For longer annealing times, a slightly concave shape in the curvature profiles evolves. For a quantitative analysis and more simple presentation, I calculated the average values of the curvature profiles in between $2.5 \mathrm{~mm}$ and $17.5 \mathrm{~mm}$ and plotted them against the total annealing time in Fig. $5.1 \mathrm{f}$ for both measurement directions. Please note that the errorbars only reflect the uncertainty in measurement of the average curvature values. They do not contain any statistical variations in the actual deformation of the sample. The average curvature is the same in both directions. It increases approximately linearly during oxidation and decreases approximately linearly afterwards. According to the simulation results of Sec. 4.2.1, the curvature decrease after complete oxidation could be explained by the influence of gravity and a constant value of viscosity. In case of a constant viscosity, the approximately linear increase in curvature during oxidation indicates an approximately constant value of integrated stress according to the viscoelastic Stoney Eq. (4.7).

To estimate the integrated film stress that causes the curvature increase, I first estimated the viscosity of the sample at the annealing temperature by a linear fit to the average curvatures in dependence on annealing time after oxidation is complete 


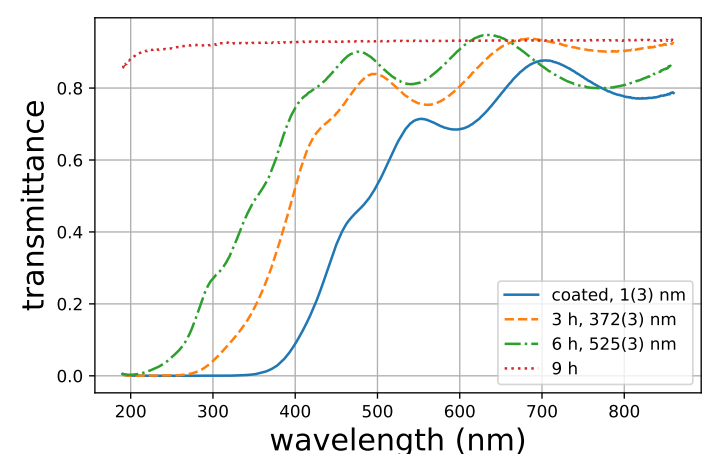

(a)

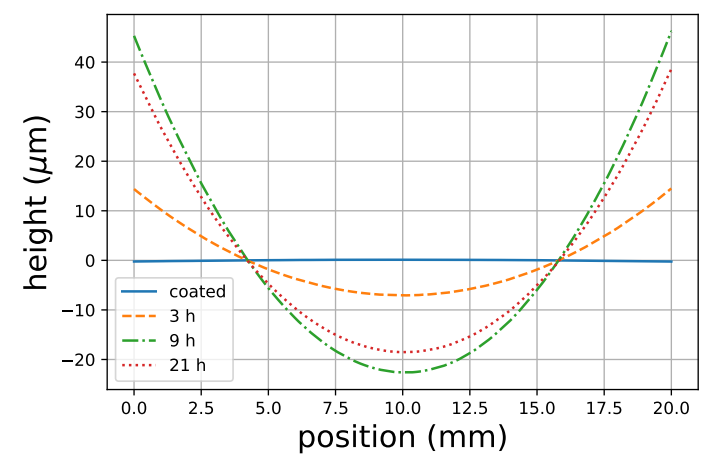

(c)

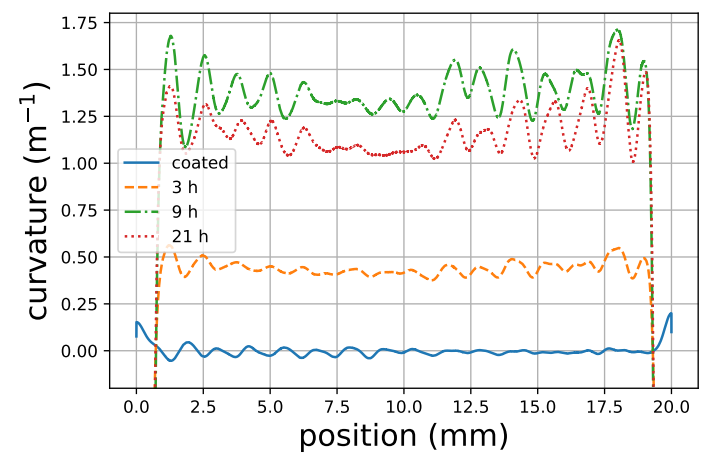

(e)

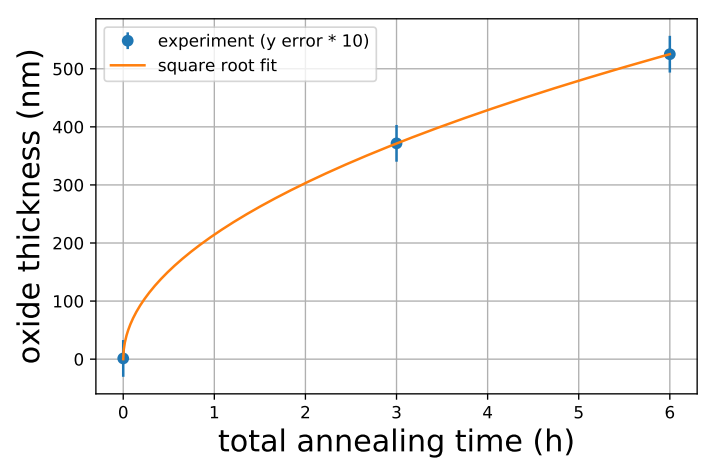

(b)

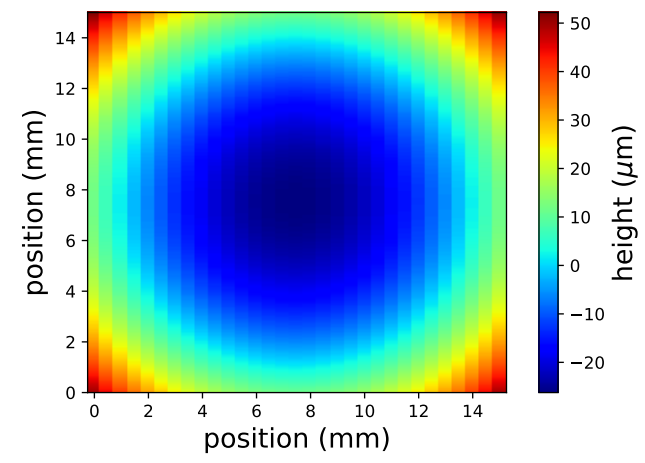

(d) After $9 \mathrm{~h}$ of annealing.

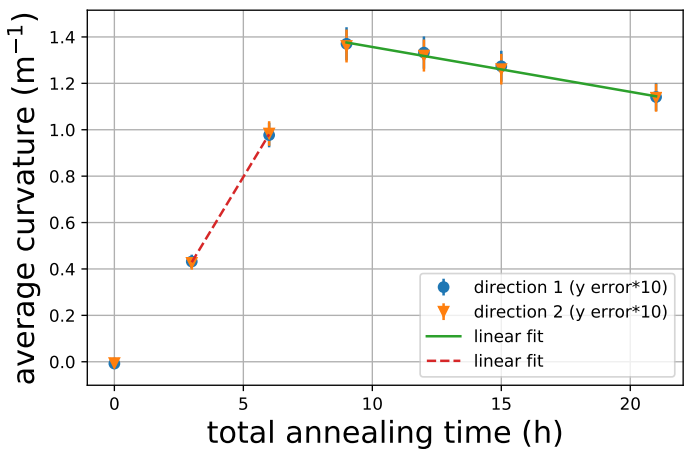

(f)

Figure 5.1: Experimental results for a sample with a continuous film of 500(6) nm silicon suboxide on a $1 \mathrm{~mm}$ thick substrate of Heraeus Suprasil. The sample was annealed at $1120(1)^{\circ} \mathrm{C}$ in ambient air in horizontal orientation. (a) The transmission spectra of the sample after the specified total annealing time. The thickness of the $\mathrm{SiO}_{2}$ oxide layer is also given. (b) The oxide thickness plotted in dependence on annealing time and fitted by assuming a diffusion limited oxidation behavior. (c) Height profiles of the non-coated side of the sample. (d) A height map of the noncoated side after $9 \mathrm{~h}$ of total annealing time. (e) The curvature profiles corresponding to the height profiles of (c). (f) The average curvature values calculated from the curvature profiles in two lateral directions in dependence on the annealing time. The error of the oxide thickness in (b) and of the curvature values in (f) has been magnified by a factor of ten for better visibility. 
(Fig. 5.1f). The fit resulted in a slope of $m_{\text {sag }}=-19.3 \cdot 10^{-3}(\mathrm{~m} \cdot \mathrm{h})^{-1}$. I achieved a similar value of $m_{\text {sag }}=-20.6 \cdot 10^{-3}(\mathrm{~m} \cdot \mathrm{h})^{-1}$ by a linear viscoelastic simulation, like the one in section 4.2.1, for a bulk modulus of $K=43 \mathrm{GPa}$, a shear modulus of $G=33 \mathrm{GPa}$, both extracted from Fig. 11 of 159 for a temperature of $1400 \mathrm{~K}$ $\left(=1127^{\circ} \mathrm{C}\right)$, and a viscosity of $\eta_{s}=2.2 \cdot 10^{11} \mathrm{~Pa} \cdot \mathrm{s}$. This value of viscosity is only by a factor of 5 smaller than the value of $1 \cdot 10^{12} \mathrm{~Pa} \cdot \mathrm{s}$ expected for the annealing point of the material (see above). Therefore, the obtained viscosity value is another argument for the gravitational influence on the sample curvature. By a linear fit to the average curvatures during oxidation, I obtained a slope of $m_{o x}=184 \cdot 10^{-3}(\mathrm{~m} \cdot \mathrm{h})^{-1}$. Please note that I excluded the point at $0 \mathrm{~h}$ because of any initial processes in the substrate, like structural relaxation (cf. [118, Part 2]) or delayed elasticity (cf. [118, Part 1]), and I excluded the point at $9 \mathrm{~h}$ because of complete oxidation. Under the assumption of the same viscosity $\eta_{s}$ as obtained above for the data after complete oxidation, calculation of the time derivative of the viscoelastic Stoney Eq. (4.7), insertion of $N=t_{f} \cdot \sigma, \tau_{s}=\eta_{s} / G_{0}^{s}$ and $G_{0}^{s}=E_{s} /\left(2\left(1+\nu_{s}\right)\right)$ [40, Eq. (6.3.38)] leads to an integrated stress of

$$
N=-t_{s}^{2} \cdot \eta_{s}\left(m_{o x}-m_{\text {sag }}\right) \approx-13 \mathrm{~N} / \mathrm{m} .
$$

The minus sign stems from the fact that opposite sample sides are considered in the theory and the experiments.

To study the influence of macroscopic (lateral dimension of structure $>$ substrate thickness $t_{s}$ ) patterned films on the deformation of the samples, I applied two different patterns in laser structuring of the films. In both cases, I achieved patterning via backside ablation (cf. Fig. 1.7) of the $\mathrm{SiO}_{x}$ films with the light of an ArF excimer laser and a homogenized laser spot (cf. Fig. 2.2). The about $1 \mathrm{~mm}$ sized quadratic spots were scanned with overlap to achieve removal of larger areas. At each position, I applied one laser pulse with a fluence of about $500 \mathrm{~mJ} / \mathrm{cm}^{2}$.

Fig. 5.2 summarizes the results for a sample with a $431(8) \mathrm{nm}$ thick $\mathrm{SiO}_{x}$ film, that was removed on one half of the coated side of the sample (Fig. 5.2f). Before annealing at $1120(1)^{\circ} \mathrm{C}$, I annealed the samples at $900{ }^{\circ} \mathrm{C}$ for $6 \mathrm{~h}$ in nitrogen atmosphere for stabilization of the film. Later on in the course of this work, I abolished this step because of no observable influence on the results. I measured the height profiles, as depicted in Fig. 5.2f, across and along the film edge, and they are shown in Figs. $5.2 \mathrm{a}$ and $5.2 \mathrm{~b}$, respectively. Across the film edge, the ablated and coated regions can be clearly distinguished from another by the difference in curvature. In contrast, along the film edge, a spherical or parabolic height profile is observed.

These observations are validated by the curvature profiles plotted in Figs. 5.2c and $5.2 \mathrm{~d}$ for the directions across and along the film edge, respectively. Across the film edge, a change in sign of the curvature can be observed. In the ablated region, the curvature is negative and declines with decreasing distance to the center of the surface area at $10 \mathrm{~mm}$, while in the coated region there is a homogeneous curvature with positive sign. Along the film edge, the curvature is mostly homogeneous but develops a slight concave profile with increasing annealing time. Interestingly, the average curvature, calculated as described above, is almost the same for both directions. This is shown in Fig. 5.2e, where it is plotted against the total annealing time. It can further be observed that the average curvature increases up to $9 \mathrm{~h}$ of 


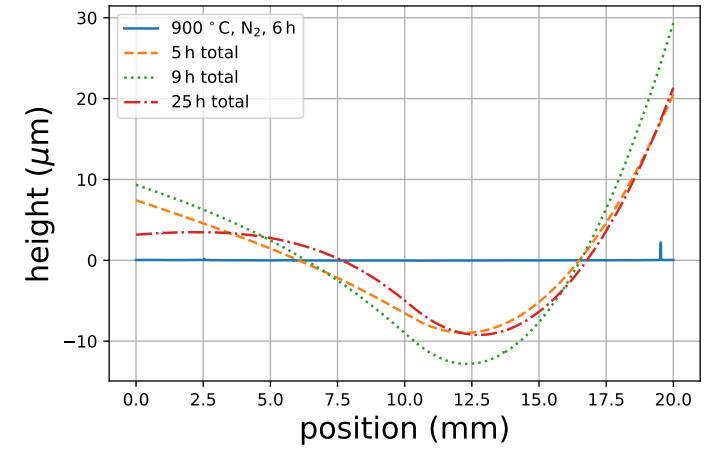

(a) across film edge

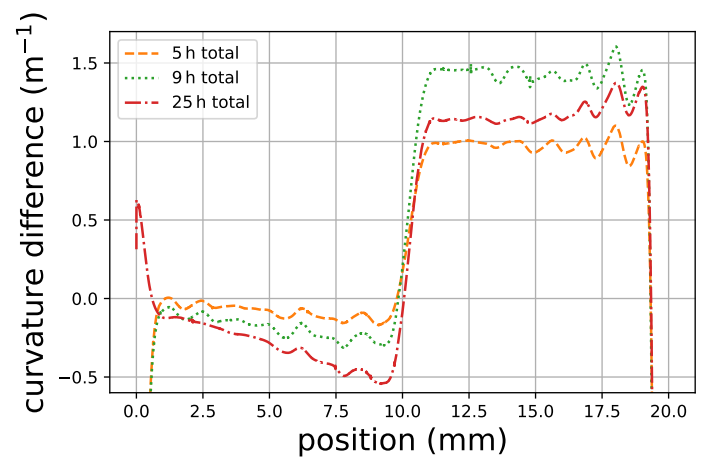

(c) across film edge

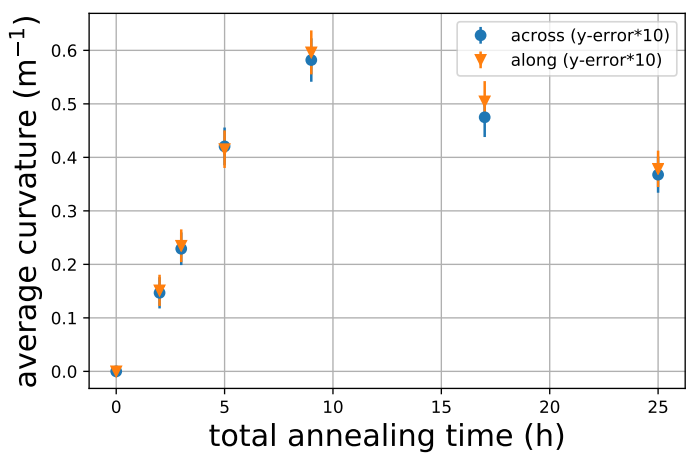

(e)

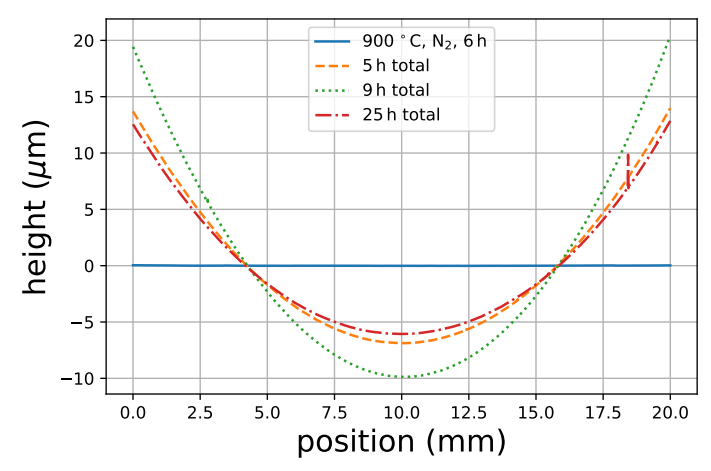

(b) along film edge

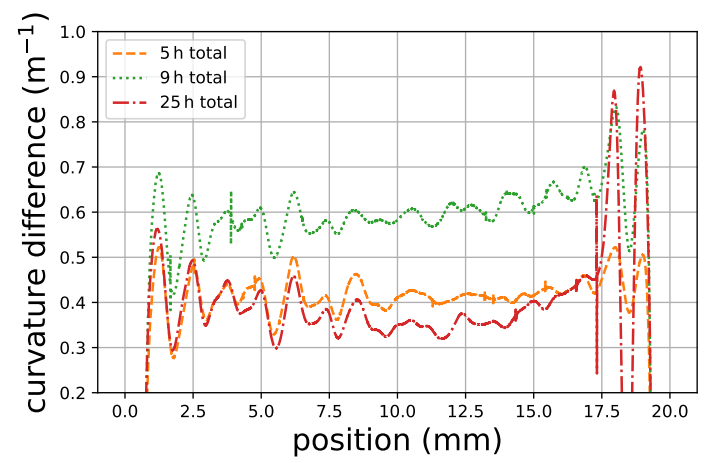

(d) along film edge

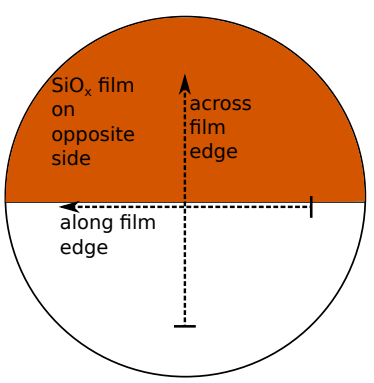

(f)

Figure 5.2: Experimental results for deformation of the non-coated side of a $1 \mathrm{~mm}$ thick Heraeus Suprasil plate by a $431(8) \mathrm{nm}$ thick film of silicon suboxide that has been removed on one half of the coated side of the sample. The sample was annealed at $1120(1){ }^{\circ} \mathrm{C}$ in ambient air in horizontal orientation. For selected annealing steps, the height profiles across the film edge (a) and along the film edge (b) are shown. The corresponding curvature profiles are plotted in (c) and (d), respectively. The average curvature difference is plotted as a function of the total annealing time in (e). Please note that the error of the curvature values is magnified by a factor of ten for better visibility. (f) Scheme of the view on the non-coated sample side. The measurement directions are indicated. 
annealing time and afterwards decreases again. From the transmission spectra of the coated sample area (not shown), I can tell that the oxidation process was complete after $9 \mathrm{~h}$ of annealing.

For short annealing times, the profiles of Fig. 5.2 agree surprisingly well with the profiles from a linear elastic FE simulation, if unrealistic values for the film stress are assumed. This is demonstrated in Fig. 5.3 for a total annealing time of 5 h. For the simulation, I assumed a film thickness of $431 \mathrm{~nm}$ and a substrate thickness of $1.078 \mathrm{~mm}$, the latter measured by a micrometer gauge in the center of the surface. I calculated the hypothetical film stress of $\sigma_{f}=-32.782 \mathrm{GPa}$ from the experimental data via the arithmetic mean of the average curvature changes in both directions and the elastic Stoney equation corrected by a factor of two (3.1). For $E_{s}$ and $\nu_{s}$, I inserted the elastic properties at room temperature, as given in table A.1 in the appendix. From Fig. 5.3b, it can be seen that the experimental curvature profiles can be well reproduced by the simulation. However, there are slight deviations in the direction across the film edge: In the ablated region $(<10 \mathrm{~mm})$, the decline of the curvature is not in agreement with the simulation. Also, in the coated region $(>10 \mathrm{~mm})$, the curvature is underestimated by the simulation.

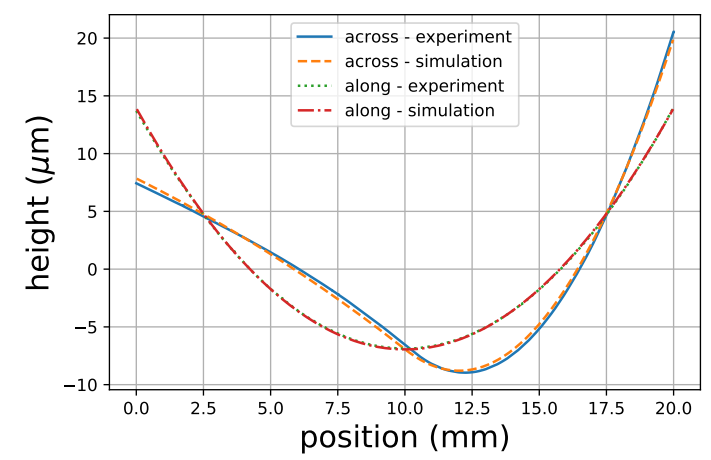

(a)

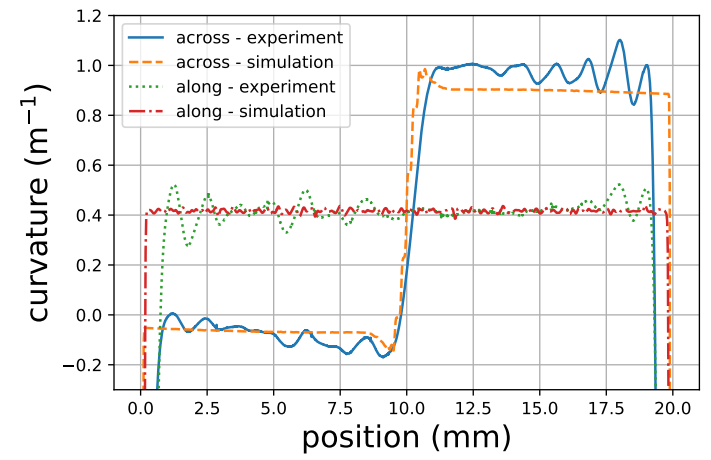

(b)

Figure 5.3: The experimental data for a total annealing time of $5 \mathrm{~h}$ of Fig. 5.2 compared to the results of a linear elastic finite element simulation. In the simulation a film stress of $\sigma=-32.782 \mathrm{GPa}$ was applied.

These differences between experiment and linear elastic simulation become more pronounced at larger total annealing times, as can be seen in Fig. 5.4, where the profiles after $25 \mathrm{~h}$ of total annealing time are compared to a linear elastic simulation based on a hypothetical film stress of $-29.268 \mathrm{GPa}$. I could achieve a better match between experiment and simulation by adding a gravitational body force to the FE simulation. For this, I assumed simply supported boundary conditions at the rim of the sample and a homogeneous mass density. I varied the hypothetical film stress and the hypothetical mass density manually for best fit to the experimental data. The best result of the simulation with gravity, for a film stress of $-54 \mathrm{GPa}$ and a mass density of $10 \mathrm{~kg} / \mathrm{cm}^{3}$, is also shown in Fig. 5.4. Across the film edge the height profile (Fig. 5.4a) is much better reproduced by the extended simulation. In Fig. $5.4 \mathrm{c}$, we see that the simulation now reproduces the slope in the curvature profile and the difference in curvature between ablated and coated region in the curvature 
profile across the film edge. Additionally, the slight concave shape of the curvature profile along the film edge can be reproduced by the simulation (Fig. 5.4d) as well. I obtained similar results for a stripe pattern of the $\mathrm{SiO}_{x}$ film. They are summarized in the appendix in section C.1.

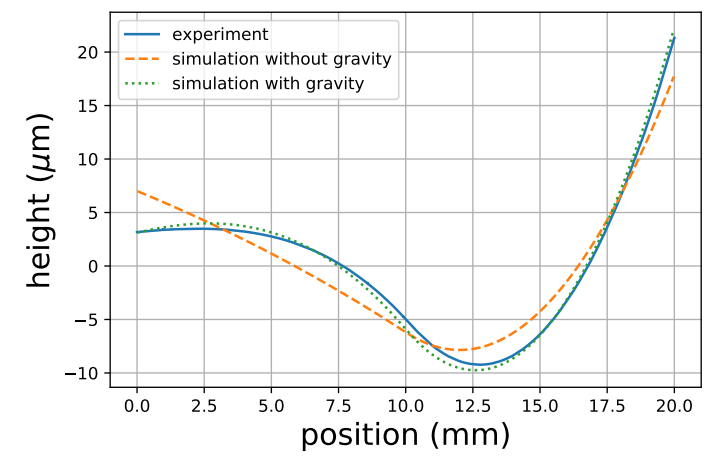

(a) across film edge

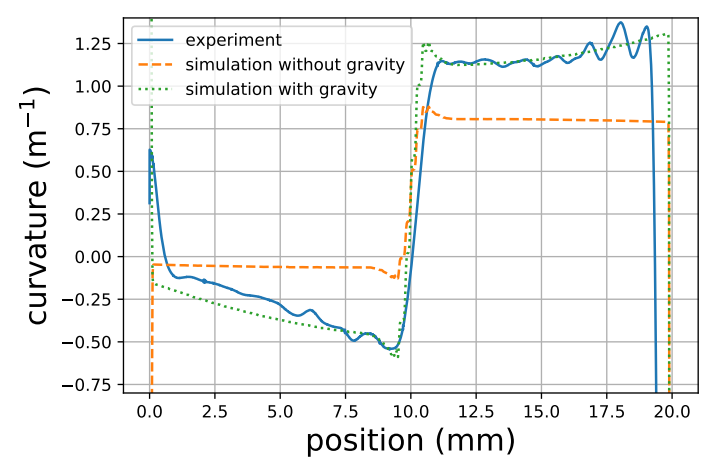

(c) across film edge

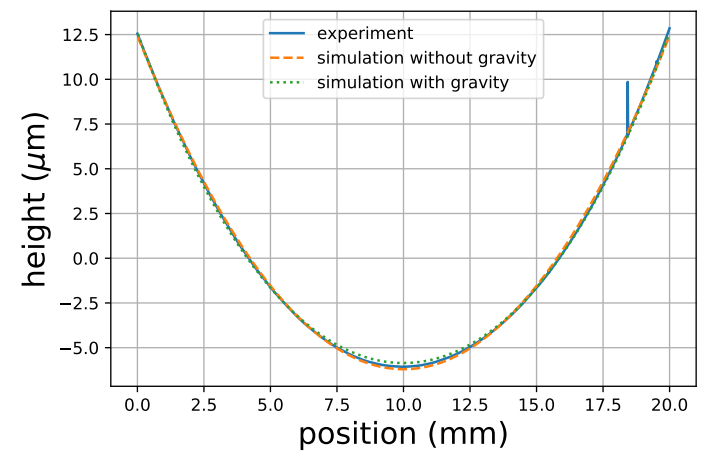

(b) along film edge

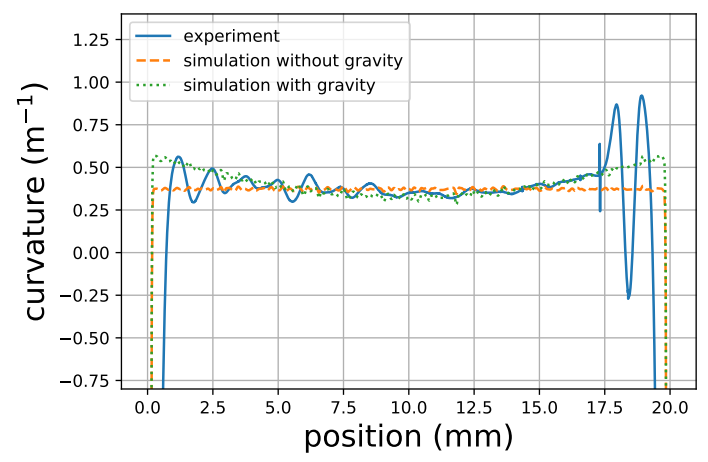

(d) along film edge

Figure 5.4: The experimental data for a total annealing time of $25 \mathrm{~h}$ of Fig. 5.2 compared to the results of linear elastic finite element simulations with and without a gravitational body force. In the case with gravity, support of the sample at its lower edge was assumed. The film stress and the mass density have been manually adjusted for best fit to the experimental data in direction along the film edge.

It has to be emphasized again that the gigantic absolute values of stress and mass density are caused by assumption of an elastic deformation, which is obviously not the right physical description. However, the good agreement between the experimental results and the elastic solution demonstrates that the elastic solution can be used for a prediction of the qualitative deformation for a specific film pattern. This finding can be understood in light of the correspondence principle (or quasi-elastic approach), which is an analytic approach for obtaining an approximate viscoelastic solution from the elastic solution by replacing an elastic modulus with a viscoelastic (time-dependent) modulus (cf. [118, pp. 295-298] or [40, Sec. 9.3.3]). 


\subsubsection{Annealing in air at $1120^{\circ} \mathrm{C}$ in vertical orientation}

The results of the previous section indicate that gravitational sag has a major influence on the deformation of substrates of Heraeus Suprasil due to a laser patterned film of $\mathrm{SiO}_{x}$. To further validate this assumption and to reduce the presumed gravitational influence, I studied annealing in vertical orientation, which I simply achieved by clamping the bottom rim of the sample between two bars of fused silica, as shown in Fig. 2.9b. I annealed the samples, as in the preceding section, in air at $1120(1)^{\circ} \mathrm{C}$.

Fig. 5.5 summarizes the results for a $1 \mathrm{~mm}$ thick substrate coated with a 505(4) nm thick film of $\mathrm{SiO}_{x}$. I removed the film by laser ablation on one half of the coated surface, as in Fig. 5.2f. The results of a similar sample with a film patterned in stripes are summarized in the appendix in Sec. C.2. During annealing, the sample was oriented in such a way that the resulting film edge lay on a horizontal line and the still coated area was farthest away from the underlying plate. The qualitative deformation is similar to the one shown in Fig. 5.2 for a comparable sample annealed in horizontal orientation: Across the film edge, the ablated $(<10 \mathrm{~mm})$ and still coated $(>10 \mathrm{~mm})$ areas can be distinguished by a negative and positive curvature, respectively, and by different magnitudes of curvature. Along the film edge, an almost homogeneous curvature is observed.

However, there are significant differences to the results of Fig. 5.2 in the evolution of the deformation in the direction across the film edge. From the transmission spectra of the coated area of the sample (not shown), I can tell that the oxidation was almost complete after $9 \mathrm{~h}$ and certainly complete after $13 \mathrm{~h}$ of annealing. From Figs. 5.1f and $5.2 \mathrm{e}$ of the preceding section, one would have expected an overall decrease in curvature after oxidation is complete. However, in Fig. 5.5e, we observe a curvature increase in direction across the film edge and a decrease in direction along the film edge. This difference could be explained by a different direction of gravity. In horizontal orientation gravity points in normal direction to the surfaces of the plate, while in the present experiment it pointed in the direction across the film edge (cf. Fig. 2.9). Another difference to the case of horizontal orientation in Fig. 5.2 lies in the slope of the curvature in dependence on total annealing time. By comparison of Fig. $5.2 \mathrm{e}$ and Fig. $5.5 \mathrm{e}$ we find that during the oxidation the curvature change is more than twice as fast in vertical orientation than in horizontal orientation, although the film thickness is comparable. The reason for this large difference in slopes is unknown.

Fig. 5.6 shows a comparison of the experimental results after a total of $9 \mathrm{~h}$ and $33 \mathrm{~h}$ of annealing with the results of a linear elastic FE simulation following the same approach as in the preceding section. For the simulation, I calculated hypothetical film stresses of -118.154 and $-129.234 \mathrm{GPa}$ for $9 \mathrm{~h}$ and $33 \mathrm{~h}$, respectively, and I did not consider the gravitational influence. For a total annealing time of $9 \mathrm{~h}$ (Figs. $5.6 \mathrm{a}$ and $5.6 \mathrm{~b}$, qualitatively similar results are obtained as in the case of horizontal orientation and a total annealing time of $5 \mathrm{~h}$ (Fig. 5.3). The height profiles are fairly well reproduced by the simulation, although for the curvature profiles, the magnitude of the curvature across the film edge is underestimated by the simulation.

In the case of a total annealing time of $33 \mathrm{~h}$, there is a large difference between 


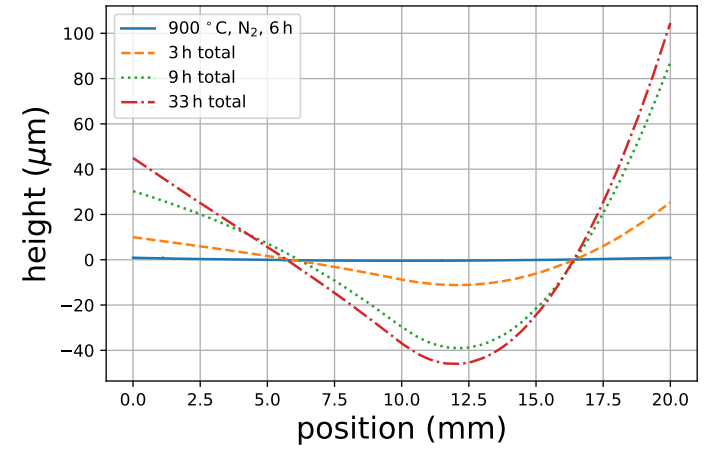

(a) across film edge

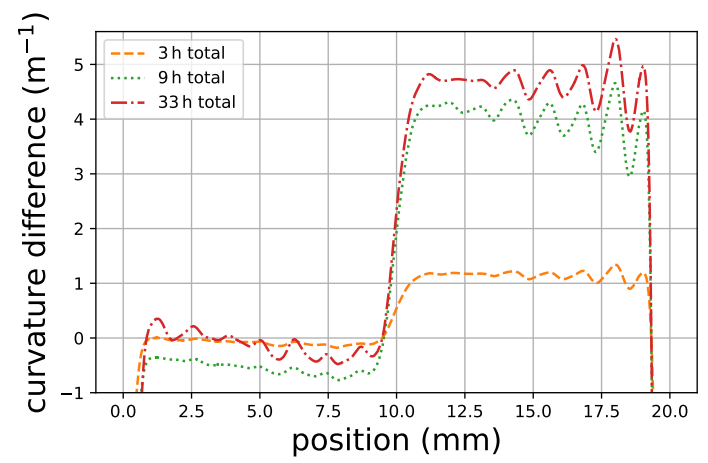

(c) across film edge

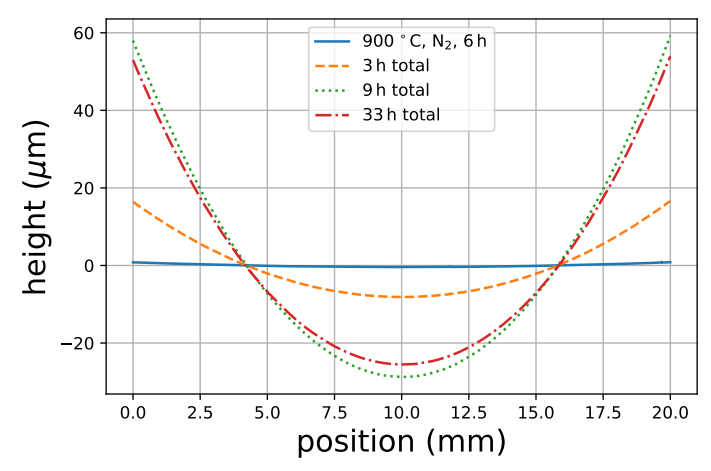

(b) along film edge

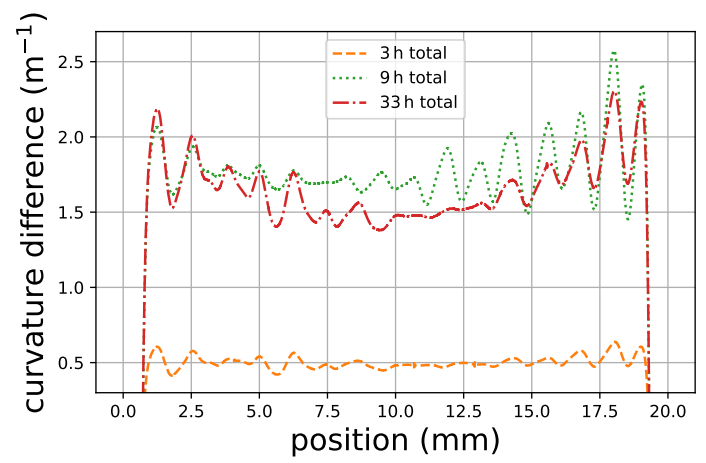

(d) along film edge

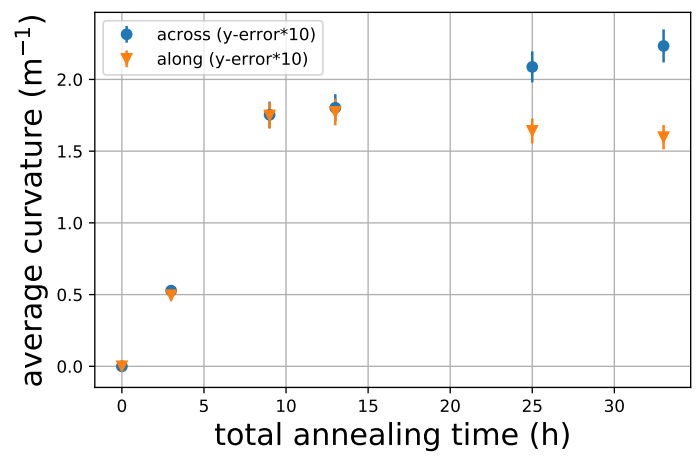

(e)

Figure 5.5: Experimental results for the deformation of the non-coated side of a $1 \mathrm{~mm}$ thick Heraeus Suprasil plate covered by a 505(4) nm thick film of silicon suboxide, that has been removed on one half of the coated side of the sample. The sample was annealed at $1120(1)^{\circ} \mathrm{C}$ in ambient air in vertical orientation. For selected annealing steps, the height profiles across the film edge (a) and along the film edge (b) are shown. The corresponding curvature profiles are plotted in (c) and (d), respectively. The curvature profiles after preliminary annealing at $900^{\circ} \mathrm{C}$ have been subtracted. The average curvature change is plotted as a function of the total annealing time in (e). The errorbars of the curvature values are magnified by a factor of 10 for better visibility. 


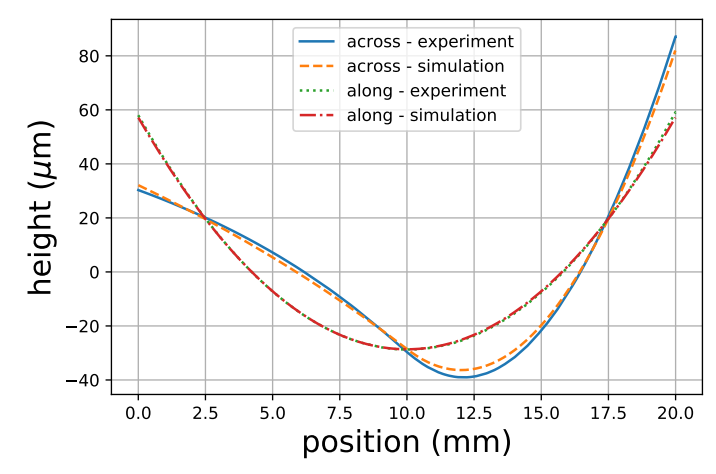

(a) $9 \mathrm{~h}$ annealing

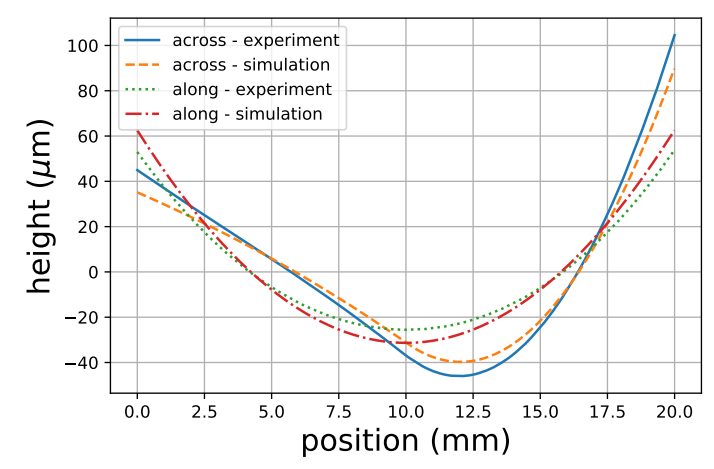

(c) $33 \mathrm{~h}$ annealing

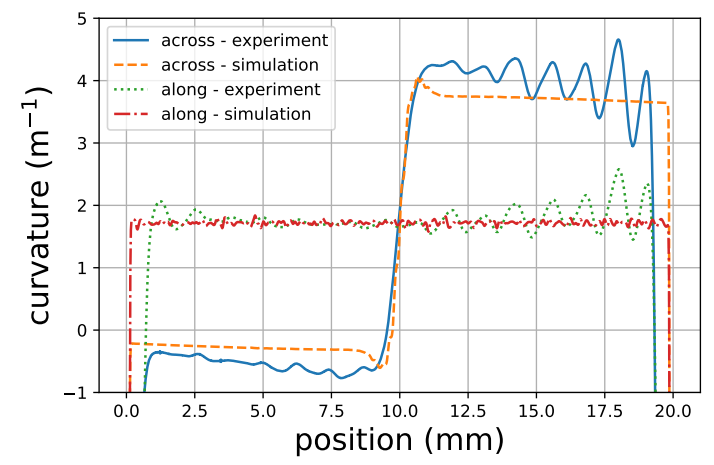

(b) $9 \mathrm{~h}$ annealing

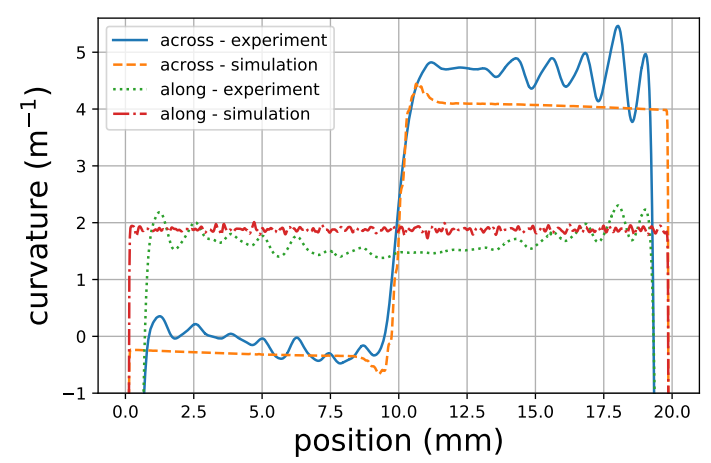

(d) $33 \mathrm{~h}$ annealing

Figure 5.6: The experimental data for a total annealing time of $9 \mathrm{~h}$ and of $33 \mathrm{~h}$ of Fig. 5.5 compared to the results of a linear elastic finite element simulation without gravity. 
the experimental height profiles and the simulation results (Fig. 5.6c). As can be seen from Fig. 5.6d, this is mostly the result of an underestimated curvature in the direction across the film edge in the coated region $(>10 \mathrm{~mm})$ and an overestimated curvature in direction along the film edge. I could not reproduce the experimental results after $33 \mathrm{~h}$ of annealing by a linear elastic simulation including gravity. This is presumably because of the undefined boundary conditions in the experiment. Nevertheless, the experiments in vertical orientation demonstrate the large influence of the sample orientation during annealing.

\subsubsection{Annealing in different atmospheres}

In the preceding sections, I demonstrated a viscous deformation of plates of fused silica covered with a film of $\mathrm{SiO}_{x}$ for annealing at $1120^{\circ} \mathrm{C}$. Because the results indicate that the deformation is mostly caused by the film stress, a better understanding of the sample behavior requires a better understanding of the film stress. Although it is reasonable to assume that oxidation of a silicon suboxide film leads to a compressively stressed film of silicon dioxide, other origins could contribute to the overall film stress. Possible additional sources for stresses are differences in the thermal expansion of film and substrate materials [160], the phase separation of the film into silicon nanoparticles inside a matrix of $\mathrm{SiO}_{x}$ with $x \approx 2$ and crystallization of the nanoparticles 161, 162.

For a better separation of these contributions, I studied the viscous deformation of two identical samples in oxygen and nitrogen flow, respectively. Each of the two samples consisted of a $1 \mathrm{~mm}$ thick substrate of Heraeus Suprasil with a diameter of $25 \mathrm{~mm}$ covered on one side by a continuous film of $\mathrm{SiO}_{x}$. I determined the film thickness of the samples by tactile profilometry to be $513(9) \mathrm{nm}$ and $505(4) \mathrm{nm}$, respectively.

I annealed the samples at $1060(10)^{\circ} \mathrm{C}$ in horizontal orientation in oxygen and nitrogen flow, respectively. After each annealing step, I measured the height profiles of the non-coated side and the reflection spectra of the coated sample side to determine the overall curvature change and the thickness of the silicon dioxide layer formed by oxidation, respectively. These values are plotted in Fig. 5.7 in dependence on the total annealing time.

The thickness of the silicon dioxide layer is plotted in Fig. 5.7a. For oxidation in oxygen atmosphere, the $\mathrm{SiO}_{x}$ film is fully oxidized after $10 \mathrm{~h}$ total annealing time, resulting in a measured silicon dioxide film thickness of $710(30) \mathrm{nm}$ (determined by tactile profilometry). In case of annealing in nitrogen flow, oxidation was observed, as well, but proceeded much slower than in oxygen. Presumably, oxidation was caused by residual oxidizing species. After $10 \mathrm{~h}$ of annealing, a film thickness of $62(3) \mathrm{nm}$ was reached. For the same time steps, the curvature change is shown in Fig. 5.7b, It is significantly larger for annealing in oxygen atmosphere, which indicates that the growth stress of the silicon dioxide film contributes to the sample deformation. However, while after $5 \mathrm{~h}$ of annealing the oxide thickness lies about one order of magnitude apart, the curvature change differs only by about a factor 


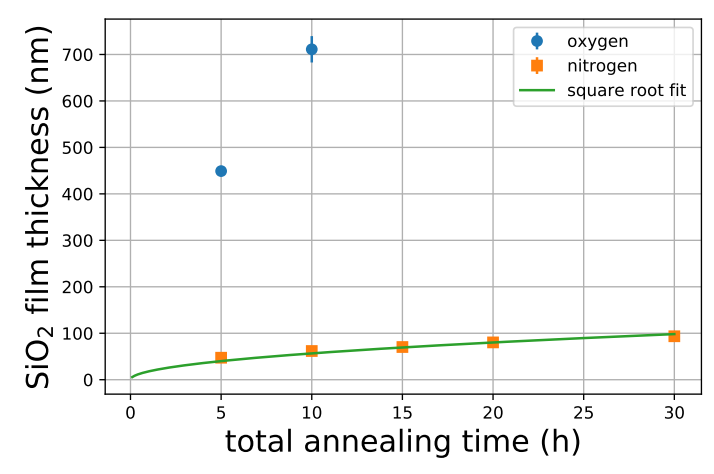

(a)

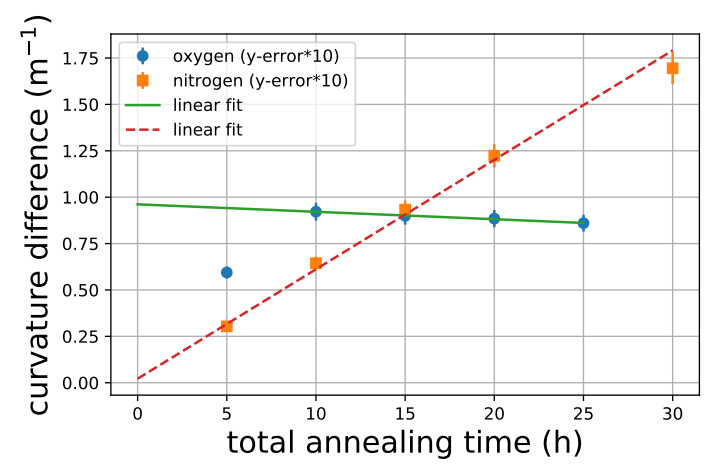

(b)

Figure 5.7: Results for the deformation of two identical samples during annealing in horizontal orientation at $1060(10)^{\circ} \mathrm{C}$ in oxygen and nitrogen atmosphere, respectively. (a) The thickness of the silicon dioxide layer formed by oxidation as a function of the total annealing time. For oxidation in flowing oxygen, the film was already completely oxidized after $10 \mathrm{~h}$ of annealing. Therefore, the final oxide thickness was determined by step height measurement via tactile profilometry. The data for annealing in nitrogen was fitted by a square root dependence. (b) The curvature difference with respect to the curvature after pre-annealing at $900^{\circ} \mathrm{C}$ as a function of the total annealing time. For estimation of the viscosity and integrated stress, two linear fits were applied to the data. The errorbars of the curvature values are magnified by a factor of 10 for better visibility.

of two in between the two samples.

The data for annealing in nitrogen is in good agreement with a linear function, as is demonstrated by a linear fit to the data in Fig. 5.7b. Under the assumption of a constant integrated stress in case of annealing in nitrogen, I estimated its magnitude the same way as in section 5.1.1 for annealing in air. First, I fitted the curvature decrease for annealing in oxygen after oxidation was complete by a linear function (Fig. 5.7b) to obtain a value for the substrate viscosity. The resulting slope of $m_{\text {sag }}=-4.01 \cdot 10^{-3} \mathrm{~m}^{-1} / \mathrm{h}$ agrees well with the slope of $-3.90 \cdot 10^{-3} \mathrm{~m}^{-1} / \mathrm{h}$ obtained by a linear viscoelastic FE simulation, like the one in section 4.2.1, for a bulk modulus of $K=43 \mathrm{GPa}$, a shear modulus of $G=42.5 \mathrm{GPa}$, which both were extracted from Fig. 11 of 159 for a temperature of $1300 \mathrm{~K}\left(1027^{\circ} \mathrm{C}\right)$, and a viscosity of $\eta_{s}=1.0 \cdot 10^{12} \mathrm{~Pa} \cdot \mathrm{s}$. The obtained viscosity $\eta_{s}$ for annealing at $1060(10){ }^{\circ} \mathrm{C}$ is larger than the viscosity value of $2.2 \cdot 10^{11} \mathrm{~Pa} \cdot \mathrm{s}$ obtained in Sec.5.1.1 for annealing at $1120(1)^{\circ} \mathrm{C}$, which can be explained by the different annealing temperatures. The linear fit to the curvature values in case of annealing in nitrogen in Fig. $5.7 \mathrm{~b}$ resulted in a slope of $m_{o x}=59.0(3) \cdot 10^{-3} \mathrm{~m} \mathrm{~h}^{-1}$. Under the assumption that the obtained viscosity value is constant and the same for annealing in oxygen and nitrogen flow, the integrated film stress for annealing in nitrogen flow is estimated via Eq. (5.1) by combination of the measured slopes $m_{s a g}$ and $m_{o x}$, the estimated viscosity $\eta_{s}$ and the measured sample thickness $t_{s}=1.084(2) \mathrm{mm}$ :

$$
N \approx-21 \mathrm{~N} / \mathrm{m} \text {. }
$$

If the film stress is generated by oxidation, the average stress inside the oxide layer $\sigma_{f}$ after $5 \mathrm{~h}$ of annealing can be estimated via dividing the above obtained value of 
$N$ by the measured oxide thickness $x_{\mathrm{SiO} 2}(5 \mathrm{~h})=62(3) \mathrm{nm}$ :

$$
\sigma_{f}(5 \mathrm{~h})=\frac{N}{x_{\mathrm{SiO} 2}(5 \mathrm{~h})} \approx-340 \mathrm{MPa} .
$$

It is interesting to see under which conditions (initial growth stress $\sigma_{f}$ and relaxation time $\tau_{f}$ of the oxide layer) the curvature evolution for annealing in nitrogen flow in Fig. $5.7 \mathrm{~b}$ can be reproduced by the viscoelastic Stoney equation including oxidation 4.19) I derived in Sec. 4.1.1. To answer this question, I first fitted the oxide thickness in dependence on the annealing time (Fig. 5.7a) by a square root dependence $x_{\mathrm{SiO} 2}=\sqrt{B_{\mathrm{dg}} \cdot t}$ on time $t$. For the parabolic rate constant, I obtained a value of $B_{\mathrm{dg}}=320 \mathrm{~nm}^{2} / \mathrm{h}$. Then, I corrected the curvature evolution for annealing in nitrogen flow in Fig. $5.7 \mathrm{~b}$ by the above determined rate of gravitational sag $m_{\text {sag }}$ and fitted it by Eq. (4.19). For the fit of Eq. (4.19), I used the above determined values of $B_{\mathrm{dg}}, K, G, \eta_{s}$ and $t_{s}$ and varied the initial growth stress $\sigma_{f}$ and the relaxation time of the $\mathrm{SiO}_{2}$ layer $\tau_{f}$. I obtained values of $\sigma_{f}=-910(40) \mathrm{MPa}$ and $\tau_{f}=7.9(5) \mathrm{h}$. They are discussed in Sec. 5.3.1. The resulting graph is plotted in Fig. 5.8 together with the experimental data after correction of the gravitational influence.

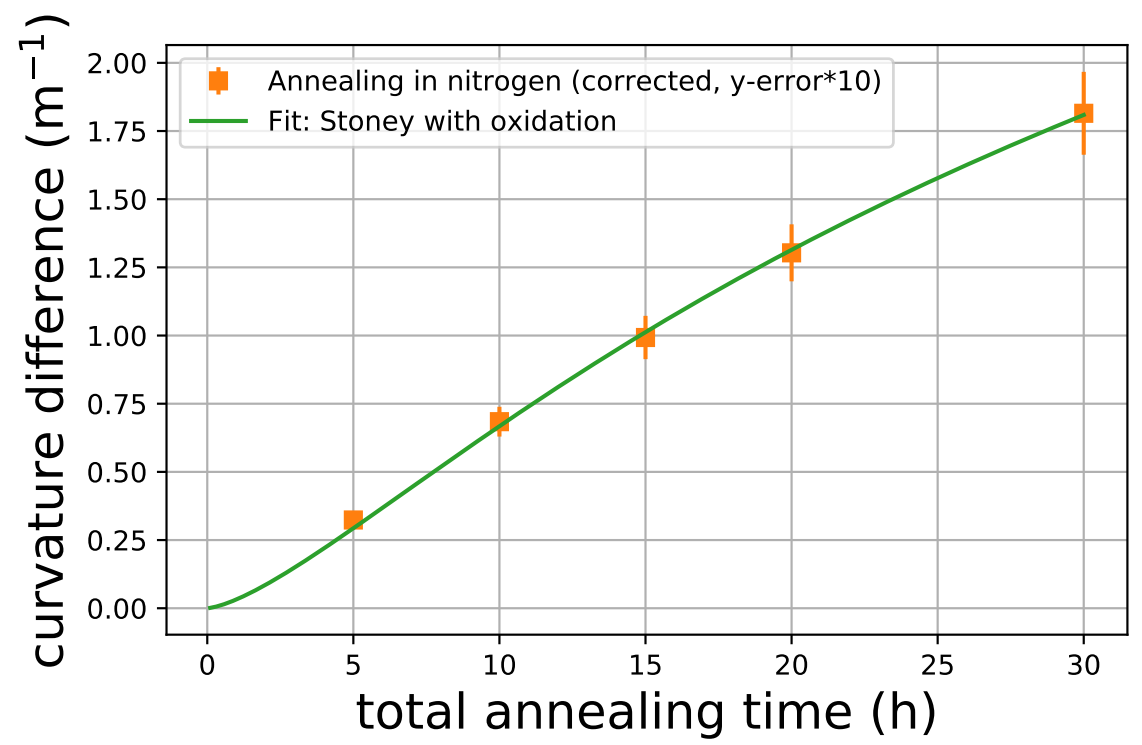

Figure 5.8: The curvature data of Fig. $5.7 \mathrm{~b}$ for annealing in nitrogen corrected by the rate of curvature change due to gravity. I fitted the data by the viscoelastic Stoney equation with parabolic oxidation 4.19 by variation of the initial growth stress $\sigma_{f}$ and the relaxation time $\tau_{f}$ (see text). The errorbars are enhanced by a factor of 10 for better visibility.

To further elucidate the origin of the film stress, I annealed samples of four different $\mathrm{SiO}_{x}$ thicknesses, roughly between 200 and $700 \mathrm{~nm}$, in nitrogen atmosphere the same way as described above. The resulting curvature after $5 \mathrm{~h}$ and $10 \mathrm{~h}$ of annealing is plotted in Fig. $5.9 \mathrm{a}$ in dependence on the film thickness. For a better comparison, I corrected the curvature by the slightly varying substrate thickness 
according to the viscoelastic Stoney Eq. (4.7). In Fig. 5.9a, it can be observed that the $\mathrm{SiO}_{x}$ film thickness has no significant influence on the absolute value of the curvature. Thus, the film stress is generated at the oxide- $\mathrm{SiO}_{x}$ or the $\mathrm{SiO}_{x}$-substrate interface.

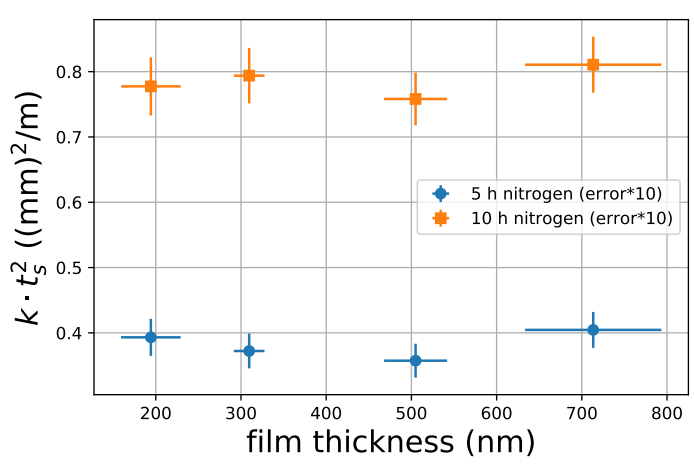

(a)

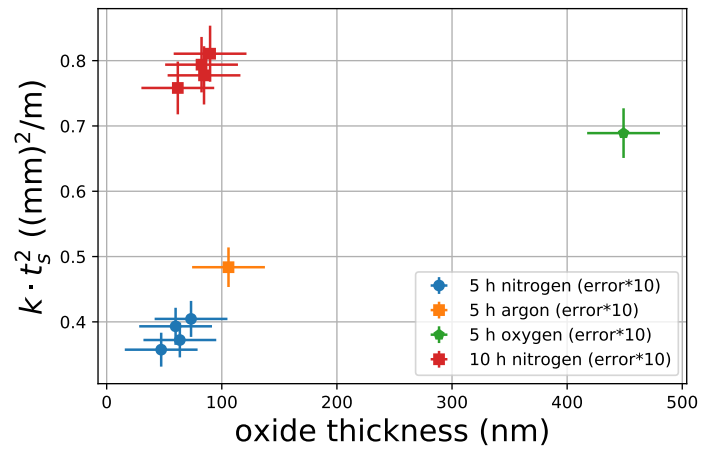

(b)

Figure 5.9: The corrected curvature in dependence on (a) the thickness of the $\mathrm{SiO}_{x}$ film for four samples after annealing in nitrogen flow and (b) on the thickness of the $\mathrm{SiO}_{2}$ oxide layer for six samples after annealing in nitrogen, argon or oxygen flow at a temperature of $1060(10)^{\circ} \mathrm{C}$. The curvature has been corrected by the substrate thickness $t_{s}$ by multiplication of $t_{s}^{2}$. The data is plotted for a total annealing duration of 5 and $10 \mathrm{~h}$. Please note, that the measurement uncertainties of the thicknesses and of the corrected curvature values have been multiplied by a factor of 10 for better visibility of the errorbars.

For further evaluation, the curvature is plotted in Fig. $5.9 \mathrm{~b}$ in dependence on the oxide thickness for samples of different film thickness annealed in nitrogen, oxygen and argon flow. For annealing for $5 \mathrm{~h}$, the curvature increases with increasing oxide thickness. This demonstrates the influence of the oxidation process on the film stress. However, there is a non-linear dependence of the curvature on the oxide thickness for annealing for $5 \mathrm{~h}$. This trend cannot be explained by the viscoelastic Stoney Eq. 4.19 for the case of parabolic oxidation and exponential relaxation because from Eq. (4.19) a linear dependence of the curvature on the oxide thickness would be expected if a similar value of $\tau_{f}$ for annealing in nitrogen, argon and oxygen flow is assumed. The linear dependence of the curvature on the oxide thickness in Eq. 4.19 can seen from the observation that the curvature depends linearly on $\sqrt{B_{\mathrm{dg}}}$, which is proportional to the oxide thickness. Therefore, the assumptions of Eq. 4.19 are too simple for a good description of the experimental results.

\subsubsection{Annealing of line patterns}

In Sec. 5.1.1, I found that the viscous deformation of the fused silica substrates due to a patterned $\mathrm{SiO}_{x}$ film is qualitatively similar to the case of an elastic deformation. Therefore, in analogy to the elastic case [38], the space of achievable viscous deformations should be significantly larger if not only equibiaxial but also antibiaxial plane stress components can be generated by patterning of the $\mathrm{SiO}_{x}$ film. It has 
been demonstrated that in the case of a stressed elastic film on an elastic substrate, a highly anisotropic deformation of the substrate, and therefore an antibiaxial plane stress component, can be induced by patterning the film into fine lines [19, 20]. This behavior has been analytically described [3], and I derived an extension of this theory in Sec. 4.1 .3 for the case of a simple linear viscoelastic substrate. The derived Eq. 4.45 suggests that the curvature ratio $k_{s} / k_{p}$, of average curvatures $k_{s}$ and $k_{p}$ in directions across and along the lines, respectively, is smaller for a viscoelastic substrate than for an elastic substrate, which is in agreement with the observations mentioned in [90] and unpublished results of Lukas Janos Richter (IFNano) for the same sample system studied here. In the present work, I studied the deformation of fused silica substrates due to a $\mathrm{SiO}_{x}$ film patterned into fine lines in detail for horizontal orientation of the samples during annealing at $1120(1)^{\circ} \mathrm{C}$ in air or at $1060(10)^{\circ} \mathrm{C}$ in flowing nitrogen.

\section{Annealing at $1120^{\circ} \mathrm{C}$ in air}

Substrates of Heraeus suprasil with a diameter of $25 \mathrm{~mm}$ and a thickness of $1 \mathrm{~mm}$ were coated with a $\mathrm{SiO}_{x}$ film of about $500 \mathrm{~nm}$ thicknes: ${ }^{2}$. Subsequently, I structured the films via backside ablation into a line pattern with a duty cycle of 0.5 and periods of 200,100 and $20 \mu \mathrm{m}$, respectively. The optical setup used for ablation was based on projection of a slit mask via a pair of cylinder lenses 3 , similarly to the setup in Fig. 2.1 without the field lens. I annealed the three samples with a structured film and a sample from the same deposition charge with a continuous film in time steps of $3 \mathrm{~h}$ in ambient air at a temperature of $1120(1)^{\circ} \mathrm{C}$ in horizontal sample orientation (cf. Fig. 2.9a).

From the transmission spectra of the sample with the continuous film (not shown), it can be deduced that oxidation of the $\mathrm{SiO}_{x}$ film was complete after $9 \mathrm{~h}$ of total annealing time. Fig. 5.10 shows an SEM image of the line pattern with $20 \mu \mathrm{m}$ period after $12 \mathrm{~h}$ of annealing. The lines have steep edges and continuously span the whole width of the sample.

The average curvatures of the samples in dependence on the annealing time are plotted in Fig. 5.11 in direction along the lines of the structured film (a) and across the lines (b). The curvatures of the samples with structured films are smaller than half the curvature of the sample with continuous film. By Eqs. (4.42), (4.43), derived for the viscoelastic case from the theory of Wikström et al., and a duty cycle of 0.5 roughly half the value would be expected. Additionally, the curvatures split up with increasing annealing time and become arranged according to the line period. That is, for large annealing times the curvature increases with increasing line period. In accordance to the experiments described above, the curvature along the lines decreases at large times, presumably after oxidation is complete. The same can be observed across the lines for the sample with a $200 \mu \mathrm{m}$ line period, but not for the two samples with smaller period. Interestingly, the annealing time, at which

\footnotetext{
${ }^{2}$ Deposition done by Lukas Janos Richter (IFNano).

${ }^{3}$ The setup was build by Jörg Meinertz (IFNano). The purpose of the lens pair is to reduce spherical aberrations 163 , Sec. 6.10]
} 


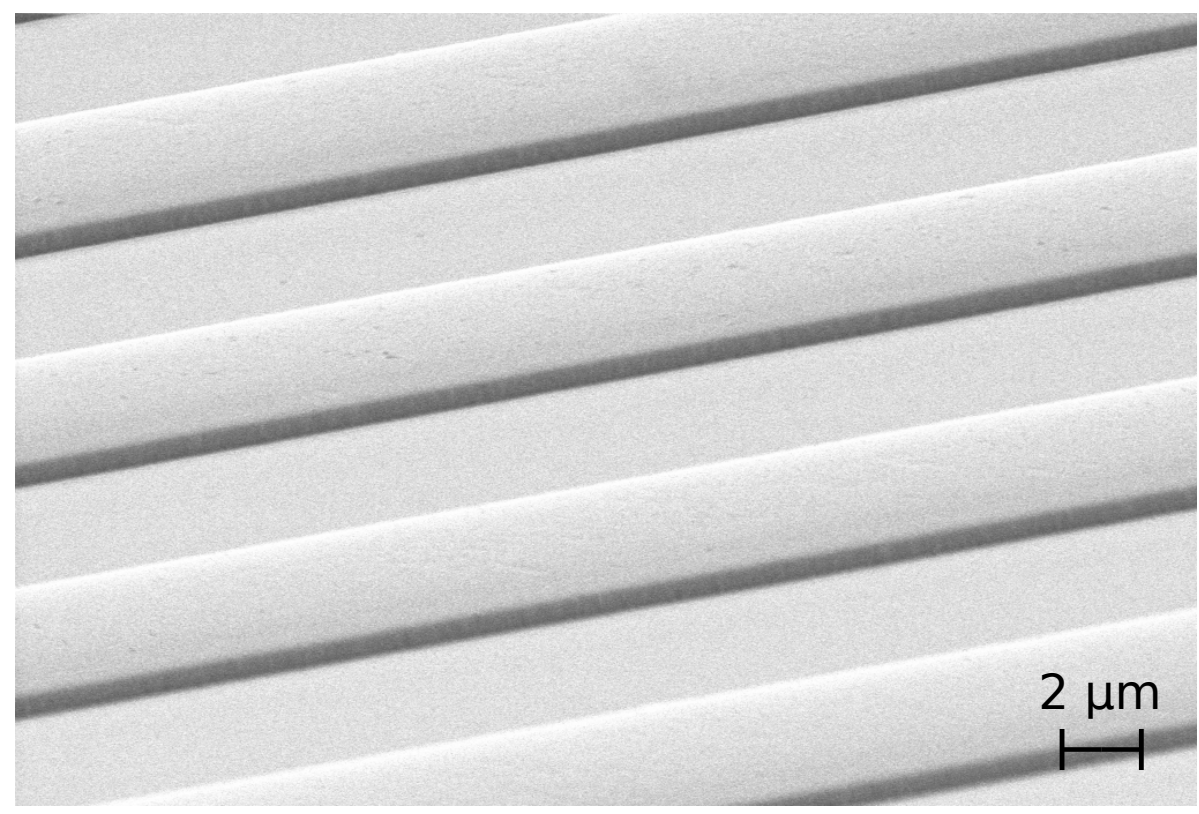

Figure 5.10: An SEM image of a $\mathrm{SiO}_{2}$ line pattern with a period of $20 \mu \mathrm{m}$ on fused silica. The pattern was produced by laser ablation of an about $500 \mathrm{~nm}$ thick $\mathrm{SiO}_{x}$ film and subsequent annealing in air at $1120(1)^{\circ} \mathrm{C}$ for $12 \mathrm{~h}$ in total. The view is inclined by $80^{\circ}$ to the surface normal. The image has been taken by Jürgen Ihlemann (IFNano).

the turning point of the curvature evolution in direction along the lines is reached, increases with increasing line period.

As a measure of the anisotropic deformation of the samples, I calculated the curvature ratio $k_{s} / k_{p}$, which is plotted against the annealing time in Fig. 5.12 . For the annealing times studied, it is always smaller than one but increases with increasing annealing time to reach a value larger than 0.9 after the last annealing step at $12 \mathrm{~h}$ of oxidation. Nevertheless, at small annealing times of 3 and $6 \mathrm{~h}$ the curvature ratio is spread over a region of about 0.3 to 0.85 and increases with increasing line period as would be expected from the elastic and linear viscoelastic Wikström theory (Sec. 4.1.3). The increase in curvature ratio with time could be partially explained by the decrease of effective aspect ratio of the lines due to the on going oxidation. That is, that the stress distribution inside the line cross section changes. This will further be discussed in Sec. 5.3.3.

I noted that the curvature profiles of the samples gain a concave shape along the lines and a slightly convex shape across the lines with increasing annealing time. As an example, the curvature profiles after annealing for $12 \mathrm{~h}$ in total are plotted in Fig. 5.13. The non-uniform curvature profiles might be explained by gravitational sag. From Fig. 5.12 it can be seen that the curvature is still bigger along the lines than across the lines after $9 \mathrm{~h}$ of annealing. Thus, during annealing the sample presumably rests on the two opposite edges that are 'connected' by the lines. Therefore, gravitational sag will lead to a convex shape of the curvature profiles across the lines (cf. Sec. 4.2.2). The concave shape of the curvature profiles in direction along the lines could be caused by a non-perfect point bearing of the sample. According to the simulations in Sec. 4.2.1, a concave curvature profile 


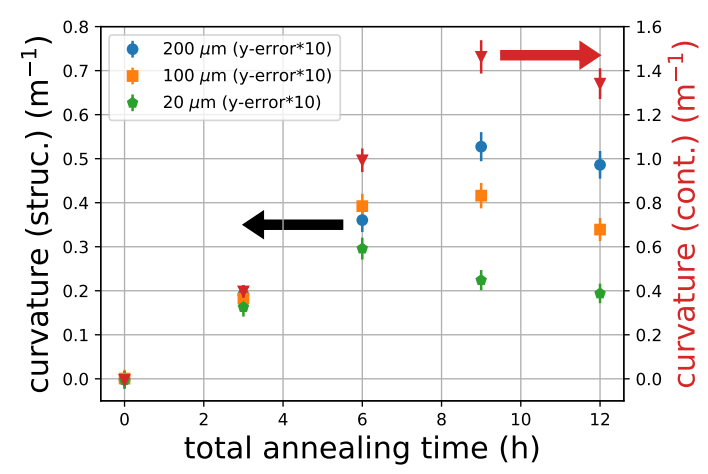

(a) Along lines

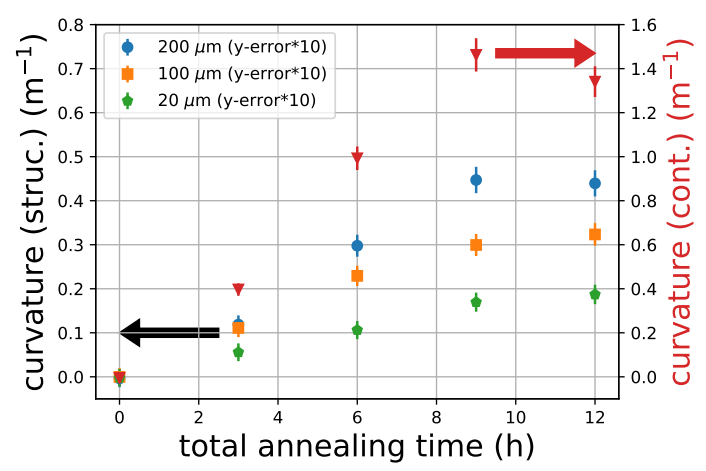

(b) Across lines

Figure 5.11: The average curvature in dependence on the total annealing time for a sample with a continuous film (right axis) and three samples with structured films (left axis). In case of the latter, the films are patterned in lines with the period specified in the legend. The curvature was measured along the lines (a) and across the lines (b). Annealing was done in horizontal orientation in air at $1120(1)^{\circ} \mathrm{C}$. The errorbars of the curvatures have been ten times magnified for better visibility.

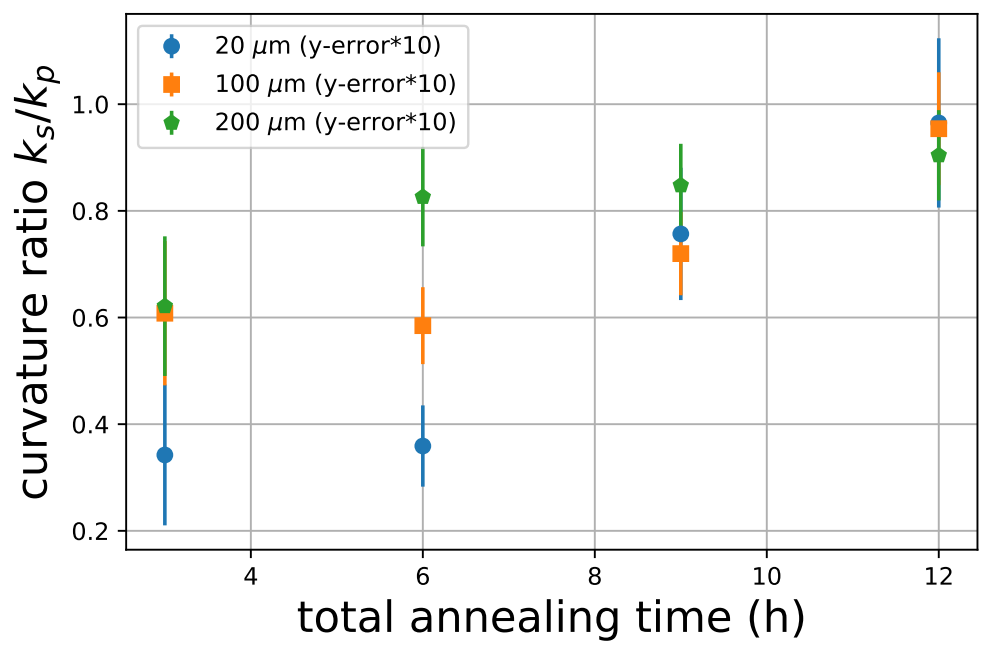

Figure 5.12: The curvature ratio $k_{s} / k_{p}$ plotted against the total annealing time for three samples with different line periods. Annealing was done in horizontal orientation of the sample in air at $1120(1)^{\circ} \mathrm{C}$. The errorbars of the curvature ratio have been ten times magnified for better visibility. 
would evolve in case of a bearing at the whole rim. Maybe, the actual boundary conditions in the experiment are an intermediate state between bearing at two points and bearing at the whole rim. Possibly, the gravitational effect also contributes to the increase in curvature ratio with increasing annealing time observed in Fig. 5.12 , as it matches the curvatures in both directions to each other.

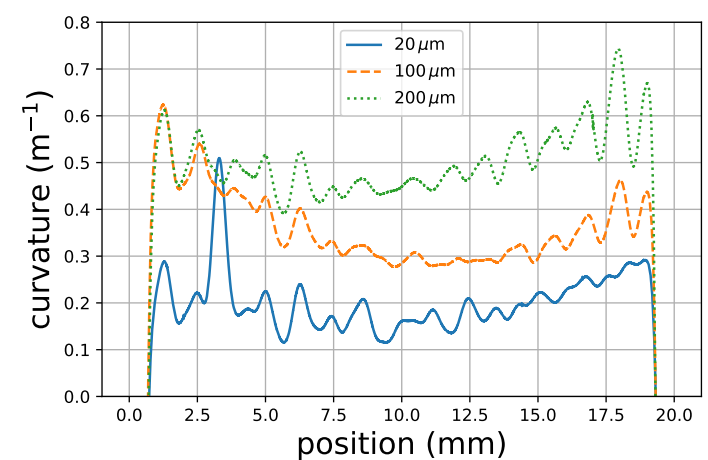

(a) Along lines

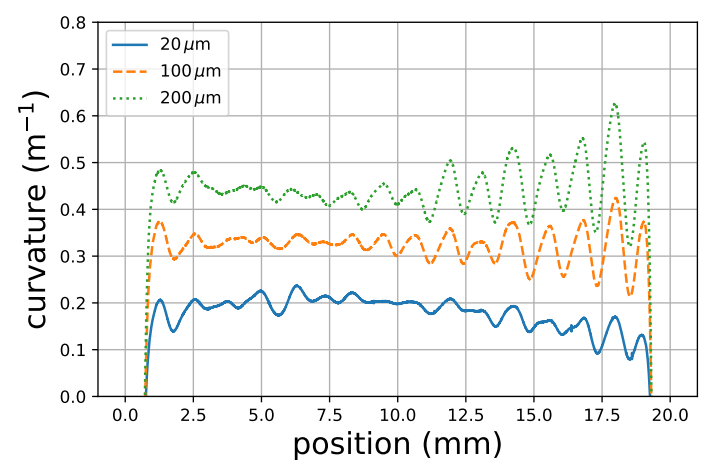

(b) Across lines

Figure 5.13: The curvature profiles along (a) and across (b) the lines for three samples with different line periods after annealing for $12 \mathrm{~h}$ in total. Annealing was done at $1120(1)^{\circ} \mathrm{C}$ in air in a horizontal sample orientation.

Between the annealing steps, I also measured height profiles across the lines on the coated surfaces of the samples via tactile profilometry. Fig. 5.14 shows the measured profiles for the sample with a period of $100 \mu \mathrm{m}$ and the sample with a period of $200 \mu \mathrm{m}$. The height of the lines increased with increasing annealing time until a total annealing time of $9 \mathrm{~h}$ was reached, as would be expected for oxidation of $\mathrm{SiO}_{x}$ [154]. Interestingly, the cross section of the lines was smoothed during annealing. That is, the samples with 20 (not shown) and $100 \mu \mathrm{m}$ period developed a round convex top, while the sample with $200 \mu \mathrm{m}$ period developed two smooth peaks. Also interesting, the ablated surface between the lines became convex and developed deep dips close to the edges of the lines.

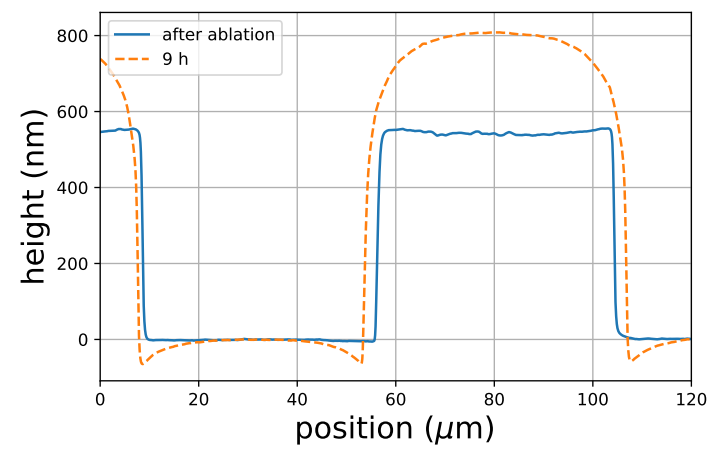

(a) $100 \mu \mathrm{m}$ period

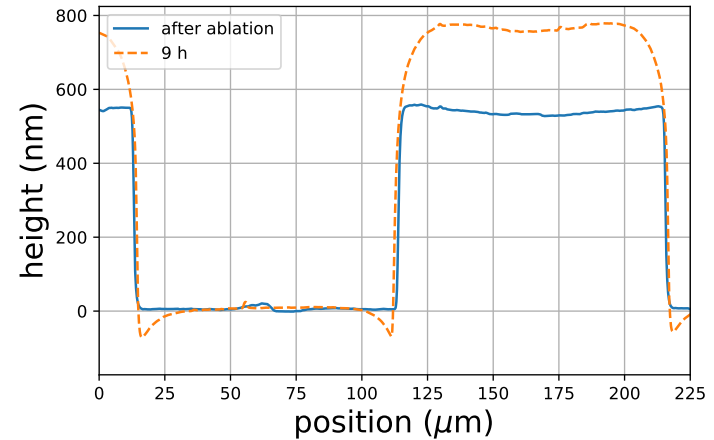

(b) $200 \mu \mathrm{m}$ period

Figure 5.14: Height profiles of the line structures with 100 (a) and $200 \mu \mathrm{m}$ (b) period after ablation and after annealing for $9 \mathrm{~h}$ in total in air at $1120(1)^{\circ} \mathrm{C}$. The measurement direction is across the lines. 
To comprehend the experimentally observed deformation of the lines, I conducted a linear elastic FE simulation. In the simulation, I considered a volume segment of length of the line period, $10 \mu \mathrm{m}$ depth and $5 \mu \mathrm{m}$ thickness of the substrate (Fig. 5.15a). I assumed the line to have a width of half the period and to span the whole depth of the substrate segment. Periodic boundary conditions were applied on all four sides of the segment under consideration. Further, I assumed a compressive lateral stress of the hypothetical value of $-20 \mathrm{GPa}$ for the line, and I assumed the elastic material properties of fused silica at room temperature for film and substrate.

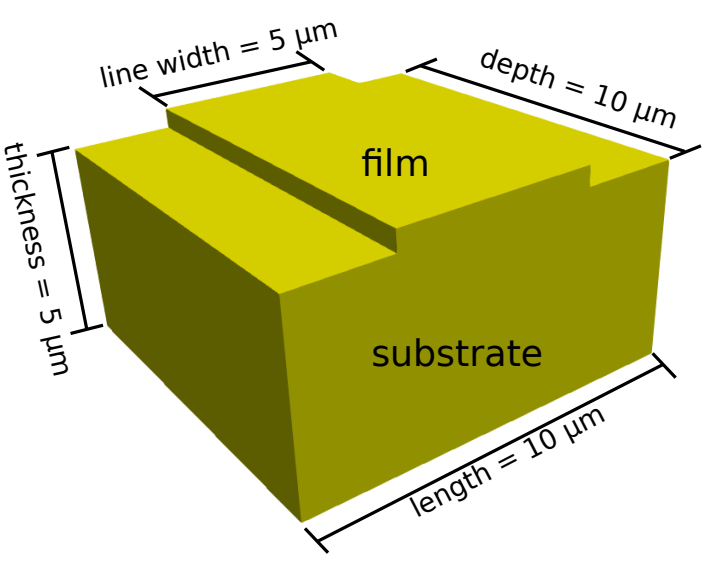

(a)

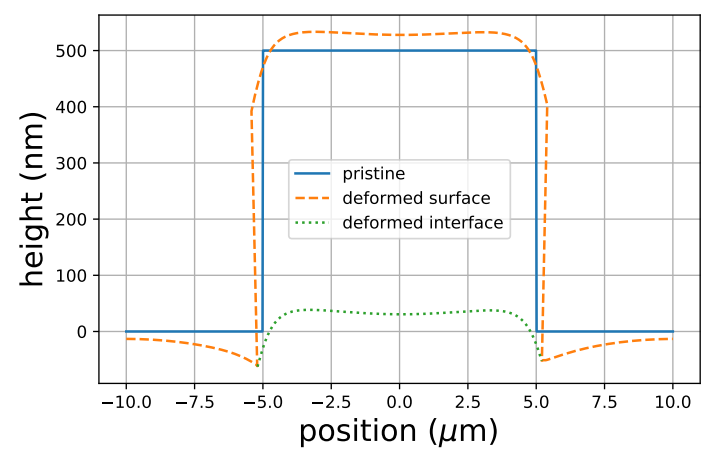

(c) $20 \mu \mathrm{m}$ period

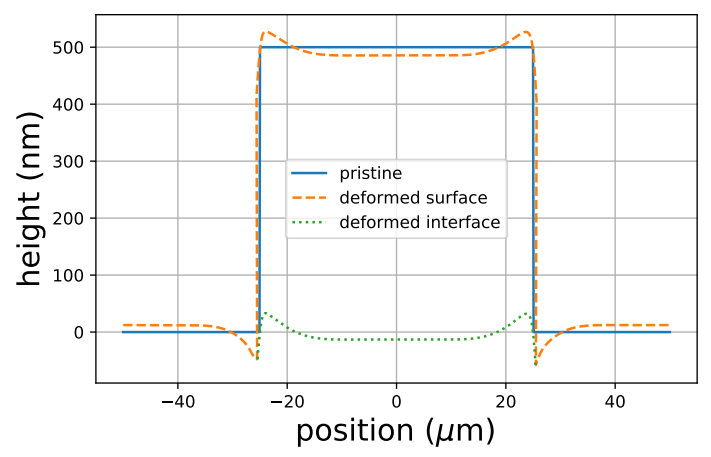

(b) $100 \mu \mathrm{m}$ period

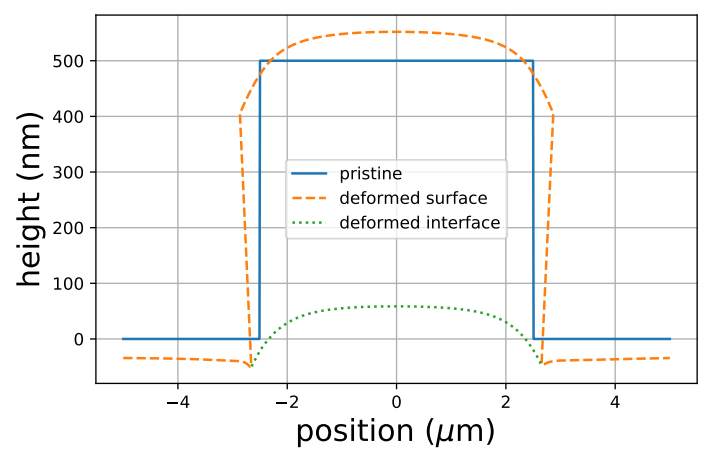

(d) $10 \mu \mathrm{m}$ period

Figure 5.15: The result of a linear elastic FE simulation for the deformation of a film line by a lateral compressive film stress of the hypothetical value of $-20 \mathrm{GPa}$. (a) The geometry for a $10 \mu \mathrm{m}$ line period. (b)-(d) The height profile before deformation, the deformed height profile and the height profile of the film-substrate interface across the line are shown for line periods of 100, 20 and $10 \mu \mathrm{m}$. The material properties of fused silica at room temperature were used.

Fig. 5.15b shows the height profiles of the surface and the film-substrate interface after deformation for a line period of $100 \mu \mathrm{m}$. The dips at the lower corners of the line in Fig. 5.14 can be reproduced. In the simulation, two peaks appear at the upper edges of the line, that remotely resemble the smooth peaks in Fig. $5.14 \mathrm{~b}$ on top of the line. The similarity becomes clear if the line width is reduced, as in Figs. $5.15 \mathrm{c}$ and $5.15 \mathrm{~d}$. The peaks move together and eventually merge, as in the simulation for a line width of $5 \mu \mathrm{m}$. This behavior could explain the single maximum observed in 
the experiment for line periods of 100 (Fig. 5.14a) and $20 \mu \mathrm{m}$. Please note that in Fig. 5.15 the top of the lines and the line-substrate interface deform the same way. Therefore, according to this explanation, the experimentally observed deformation of the lines, separate from the height increase, is a consequence of the deformation of the underlying substrate due to the film stress.

\section{Annealing at $1060(10)^{\circ} \mathbf{C}$ in nitrogen flow}

In the preceding subsection, I demonstrated that annealing of samples with a line structured $\mathrm{SiO}_{x}$ film leads to the desired anisotropic deformation of the samples. However, the samples show a complex behavior with annealing time, that could be caused by gravitational force and fast oxidation. To reduce the potential influence of oxidation, I repeated the experiment with the annealing conditions of section 5.1.3, namely slow oxidation in nitrogen flow at a temperature of $1060(10)^{\circ} \mathrm{C}$. Additionally, I increased the film thickness from about $500 \mathrm{~nm}$ to about $1100 \mathrm{~nm}$. Thereby, the oxidation rate in relation to the overall film thickness was further decreased and the anisotropic behavior should be enhanced due to an increased aspect ratio. In Sec. 5.1.3, I also demonstrated that the integrated film stress is larger for annealing in nitrogen flow at $1060(10){ }^{\circ} \mathrm{C}$ than for annealing in air at $1120(1){ }^{\circ} \mathrm{C}$ (cf. Sec. 5.1.1), which are the conditions of the preceding subsection. Therefore, annealing in nitrogen flow at $1060(10)^{\circ} \mathrm{C}$ should lead to a relatively smaller influence of gravity on the deformation. I used a similar optical setup as in the preceding subsection for structuring of the $\mathrm{SiO}_{x}$ film. However, instead of the lens pair, I used a single cylindrical lens. To compensate for a smaller output pulse energy of a different laser (LPX-Pro instead of LPX 300), I also introduced a spherical field lens (cf. Fig. 2.1). As a consequence, two lines had to be stitched together for spanning the whole width of the sample.

For reference, I annealed a sample with a continuous (non-structured) film the same way as three samples with structured films, and I measured the $\mathrm{SiO}_{2}$ oxide thickness in between the annealing steps. It increased with increasing total annealing time (not shown) and after $34 \mathrm{~h}$ a thickness of $129(3) \mathrm{nm}$ was reached. If a thickness ratio of $t_{\mathrm{SiO}_{2}} / t_{\mathrm{SiO}_{x}}=1.35$ [154] is assumed for the thickness of a $\mathrm{SiO}_{x}$ film before $\left(t_{\mathrm{SiO}_{x}}\right)$ and after $\left(t_{\mathrm{SiO}_{2}}\right)$ oxidation, the thickness of the $\mathrm{SiO}_{x}$ film should have decreased to about $1000 \mathrm{~nm}$ and the thickness of the bilayer film system $\left(\mathrm{SiO}_{2}\right.$ and $\mathrm{SiO}_{x}$ ) should have increased by about $30 \mathrm{~nm}$ after $34 \mathrm{~h}$ of annealing. Thus, only a small relative change of the aspect ratio of the line structures is expected.

The average curvatures along and across the lines are plotted in Fig. 5.16 in dependence on the total annealing time. As for annealing in air, the curvatures of the samples with structured films are much smaller than for the sample with a continuous film. If the curvatures are divided by the ones for the continuous film, a value of 0.2 is approached along the lines. Across the lines, the curvatures diverge with increasing annealing time. In contrast to the results for annealing in air, no turning point is observed at which the slope of the curvature-time curve becomes negative. This is because of the much slower oxidation process, that prevents the complete oxidation on relevant time scales. 


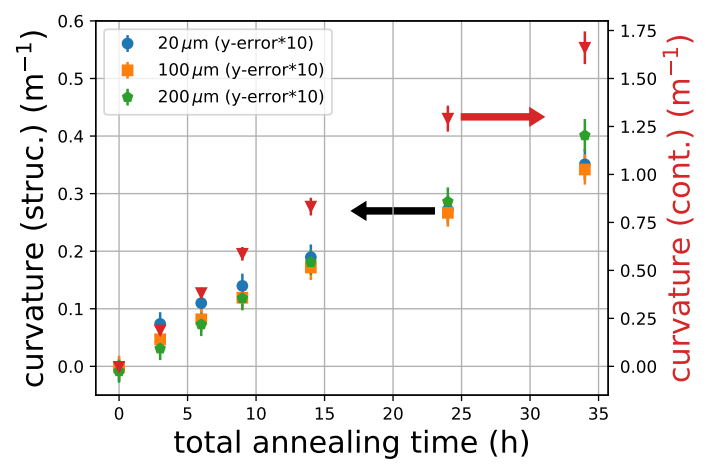

(a) Along lines

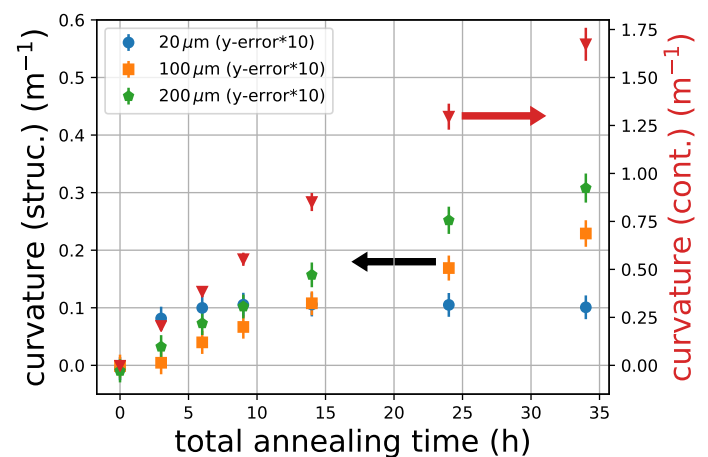

(b) Across lines

Figure 5.16: The average curvature in dependence on the total annealing time for a sample with a continuous film (right scale) and three samples with structured films (left scale). In case of the latter, the films are patterned in lines with the period given in the legend. The curvature was measured along the lines (a) and across the lines (b). Annealing was done in horizontal orientation in nitrogen flow at $1060(10)^{\circ} \mathrm{C}$. The errorbars of the curvatures have been ten times magnified for better visibility.

Fig. 5.17 shows the calculated curvature ratio $k_{s} / k_{p}$ for the same measurement values in dependence on the annealing time. As expected, for the sample with a continuous film, the curvature ratio approaches $k_{s} / k_{p} \approx 1$ with increasing annealing time. The curvature ratios of the samples with 100 and $200 \mu \mathrm{m}$ period seem to level off with increasing annealing time, while after $34 \mathrm{~h}$ of annealing the curvature ratio of the sample with $20 \mu \mathrm{m}$ line period still decreases. The behavior of the latter is a result of the fact that the curvature along the lines increases with annealing time while the curvature across the lines stays constant (cf. Fig. 5.16). Thus, if this trend continues, eventually the curvature ratio of this sample will be zero. However, with increasing annealing time the non-uniformity of the curvature profiles increased (Fig. 5.19). Since this behavior hinders the interpretation of the calculated average curvature values, the experiment was stopped after $34 \mathrm{~h}$ of total annealing time.

In Fig. 5.17, the curvature ratio increases with increasing line period at a fixed annealing time for annealing times larger than or equal to $14 \mathrm{~h}$. I calculated the aspect ratio of the line structures by dividing the thickness of the films before structuring, measured by tactile profilometry, by the width of the ridges directly after structuring, measured by optical microscopy. In Fig. 5.18, the behavior of the curvature ratios of Fig. 5.17 in dependence on the aspect ratio of the lines after annealing for $34 \mathrm{~h}$ is compared to the theoretical behavior for the case of an elastic substrate with a Poisson's ratio of $\nu_{s}=0.165$ of fused silica at room temperature and to the derived theory for a simple linear viscoelastic substrate (Eq. 4.45). In both cases, I assumed the Possoin's ratio of the elastic film to be $\nu_{f}=0.17$ of a $\mathrm{SiO}$ film [164]. This value can only serve as a rough estimate because in [164 the films were prepared differently than mine and in my case the sample was annealed at high temperature. Thus, the films presumably underwent a phase separation, and, at least for the viscoelastic equation, the Poisson's ratio at the annealing temperature should be used if it were known. Nevertheless, from Fig. 5.18 it can be seen that the 


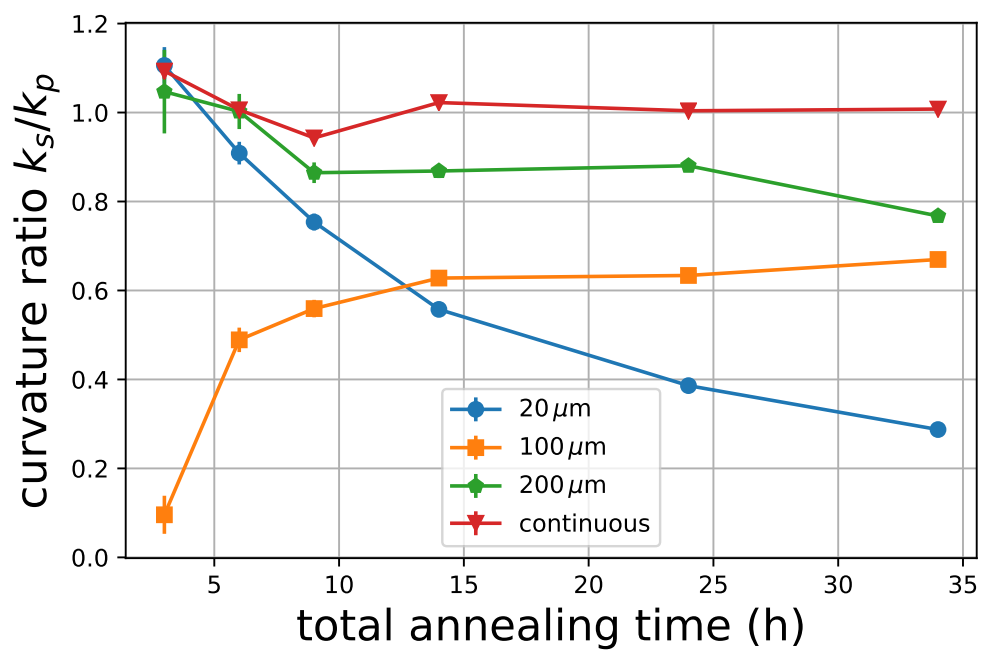

Figure 5.17: The curvature ratio $k_{s} / k_{p}$ plotted against the total annealing time for one sample with a continuous film and three samples with different line periods. Annealing was done in horizontal sample orientation in nitrogen flow at $1060(10){ }^{\circ} \mathrm{C}$.

simple linear viscoelastic theory is a better match to the experimental data than the elastic one. Additionally, if it is assumed that the curvature ratio of the sample with highest aspect ratio would further decrease to zero with increasing annealing time, as has been explained above, both theories would overestimate the experimentally obtained values.

The curvature profiles of the same data are plotted in Fig. 5.19 after annealing for $34 \mathrm{~h}$ in total. As was already noted in Fig. 5.13 for annealing in air, a concave shape evolved in direction along the lines and a convex shape evolved in direction across the lines. The convex shape in direction across the lines (Fig. 5.19b) is more pronounced for smaller periods.

To verify that the deformations of the samples are as one would expect from the height profiles in two orthogonal directions, I measured height maps of the noncoated side by tactile profilometry. In Fig. 5.20, the height maps of the sample with a continuous film (a) and of the sample with the $20 \mu \mathrm{m}$ line period (b) are shown for a total annealing time of $34 \mathrm{~h}$. For the continuous film, a spherical deformation is observed. The height map for the structured film is oriented such that the ordinate (the measurement direction) is pointing in direction across the lines. The deflection of the surface is larger along the lines than across them, which is in agreement with the measured average curvatures in Fig. 5.16. There are no higher order deformations. Thus, the deformation is as expected from the two height profiles measured in orthogonal directions. It needs to be mentioned, that, in contrast to annealing in air at $1120(1){ }^{\circ} \mathrm{C}$ (cf. Fig. 5.14), I did not observe any large deformations on a small lateral scale in the height profiles of the structured surface after annealing.

The described results for deformation by line patterned films confirm that small values of the curvature ratio $k_{s} / k_{p}$ can be reached by a line pattern. They also demonstrate that the dependence of the curvature ratio on the aspect ratio of the lines is similar to the elastic case and thermal stresses. However, they also show a 


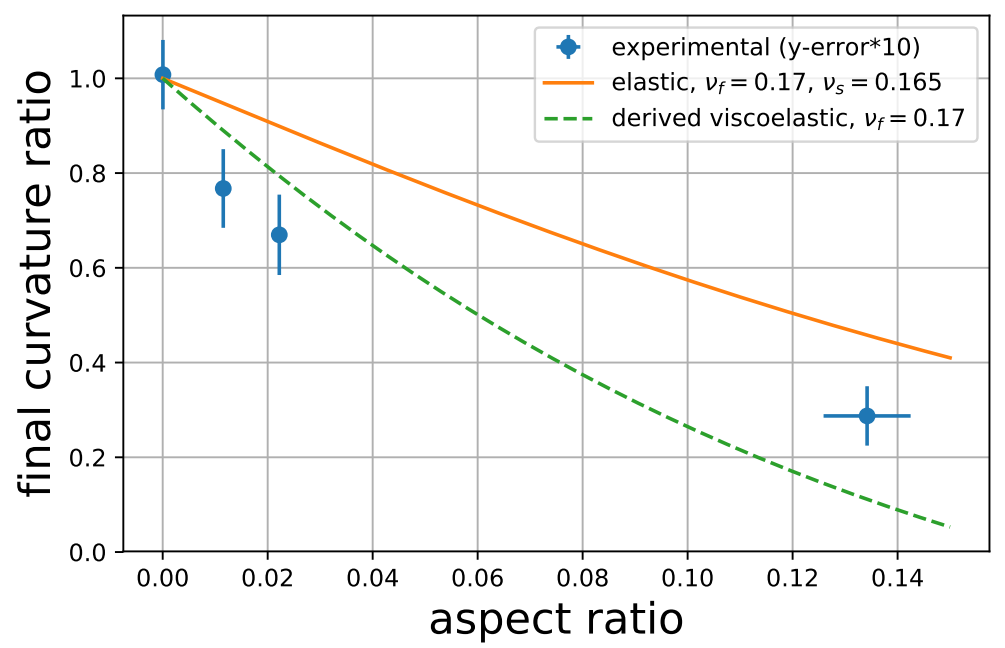

Figure 5.18: The curvature ratio $k_{s} / k_{p}$ of Fig. 5.17 after $34 \mathrm{~h}$ of annealing plotted against the aspect ratio of the lines before annealing. Annealing was done in horizontal sample orientation in nitrogen flow at $1060(10){ }^{\circ} \mathrm{C}$. The errorbars of the curvature ratio have been ten times magnified for better visibility. For comparison, the theory of Wikström et al. [3] for an elastic substrate and the extended theory for a viscoelastic substrate are plotted, as well. The stated values of the Poisson's ratios $\nu_{f}=0.17$ and $\nu_{s}=0.165$ of film and substrate, respectively, were used.

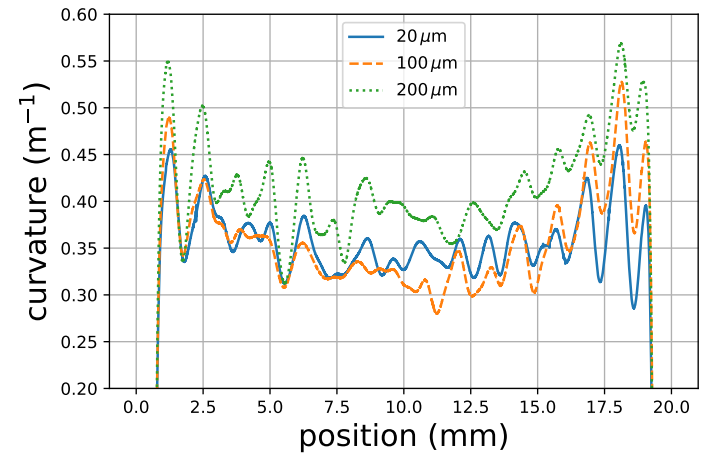

(a) Along lines

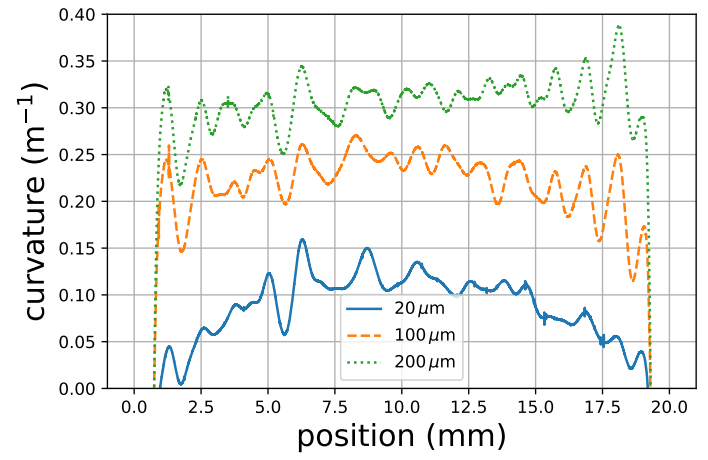

(b) Across lines

Figure 5.19: The curvature profiles along (a) and across (b) the lines for different line periods after $34 \mathrm{~h}$ of annealing in nitrogen flow. 


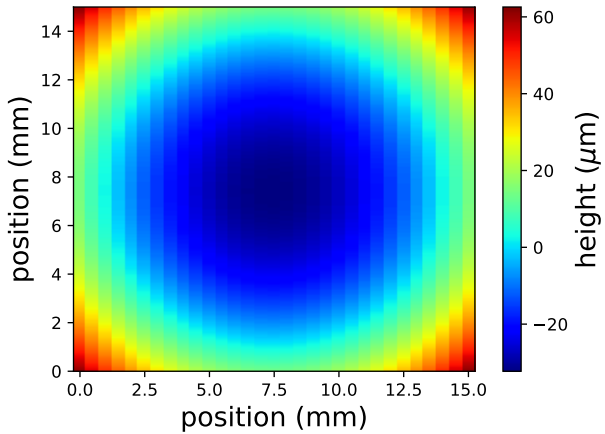

(a) Continuous film

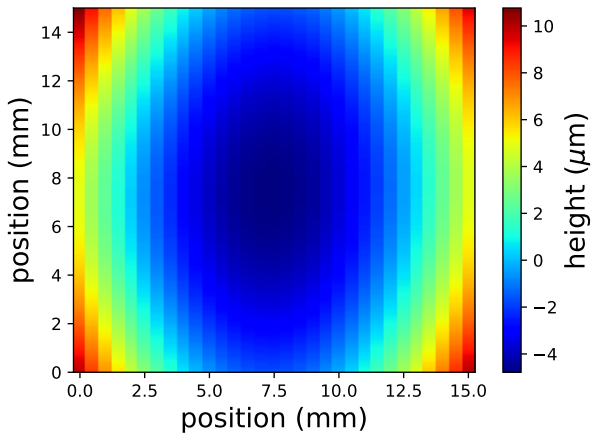

(b) $20 \mu \mathrm{m}$ line period

Figure 5.20: Height maps of the non-coated surfaces of the samples with (a) continuous $\mathrm{SiO}_{x}$ film and (b) a line structure with a period of $20 \mu \mathrm{m}$. The samples were annealed for $34 \mathrm{~h}$ at $1060(10)^{\circ} \mathrm{C}$ in nitrogen flow.

complex behavior of the curvature ratio with time. This is especially true for the case of a fast oxidation of the film and a significant gravitational influence.

\subsection{Additional results}

Section 5.1 dealt with the viscous deformation of fused silica plates at very high temperatures of 1120 and $1060^{\circ} \mathrm{C}$. I performed additional experiments at lower temperatures, at which an elastic deformation of the substrate can be observed and the oxidation proceeds at a much slower rate. The results contribute to a better understanding of the $\mathrm{SiO}_{x}$-fused silica system.

For analysis of the elastic deformation of the substrate, I reduced the substrate thickness by a factor of 4 by use of fused silica cover glasses with a thickness of about $250 \mu \mathrm{m}$. The cover glasses were coated with silicon suboxide, as described in section 2.2 . The film stress at room temperature after coating, as measured by deformation of the samples, was tensile with a magnitude of $50-110 \mathrm{MPa}$. Because I measured the deformation of the non-coated sample side, a negative curvature corresponds to a tensile (positive) film stress, and a positive curvature corresponds to a compressive (negative) film stress (Fig. 5.21).

\subsubsection{Stress change of silicon suboxide films due to anneal- ing}

To study the changes inside the $\mathrm{SiO}_{x}$ films during a temperature raise, I measured the change in transmittance and curvature at room temperature of GE124 cover glasses covered with continuous films of $\mathrm{SiO}_{x}$ due to annealing in flowing nitrogen for different annealing temperatures. Fig. $5.22 \mathrm{a}$ shows a typical behavior in dependence on the annealing temperature. I annealed a sample with a film thickness 

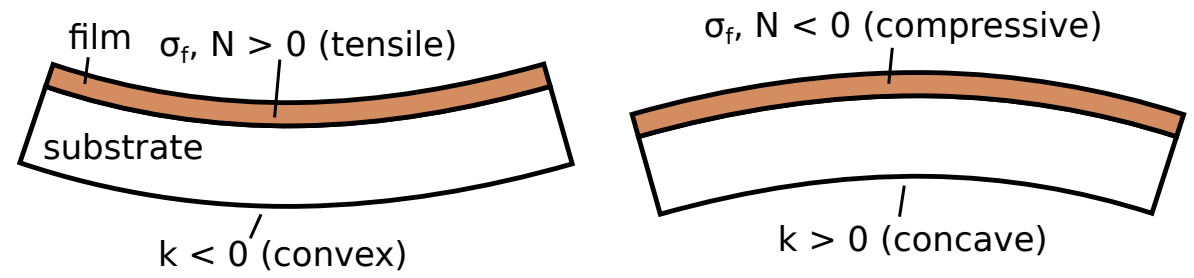

Figure 5.21: For a better understanding of the results of Sec. 5.2, the sample deformation due to a tensile (left) or compressive film stress $\sigma_{f}$ (right) is sketched. The usual convention is to denote a tensile film stress by a positive sign and a compressive film stress by a negative sign. The same holds for the integrated stress $N$. Because in Sec. 5.2 the deformation of the non-coated sample side is considered, a tensile film stress corresponds to a negative curvature $k$ and a compressive film stress corresponds to a positive curvature.

of $360(20) \mathrm{nm}$ at increasing temperatures for $6 \mathrm{~h}$ at each temperature. The overall transmission increases at small temperatures up to about $250^{\circ} \mathrm{C}$, and for annealing at $700{ }^{\circ} \mathrm{C}$ it sharply drops. This trend continuous until the transmission increases again at a temperature of $900^{\circ} \mathrm{C}$.

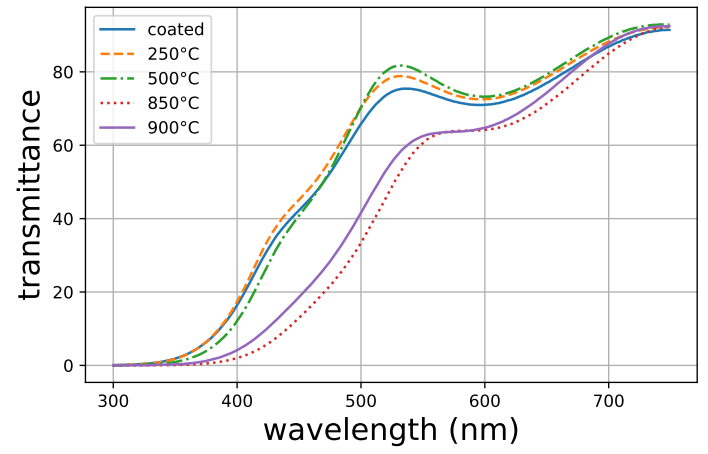

(a)

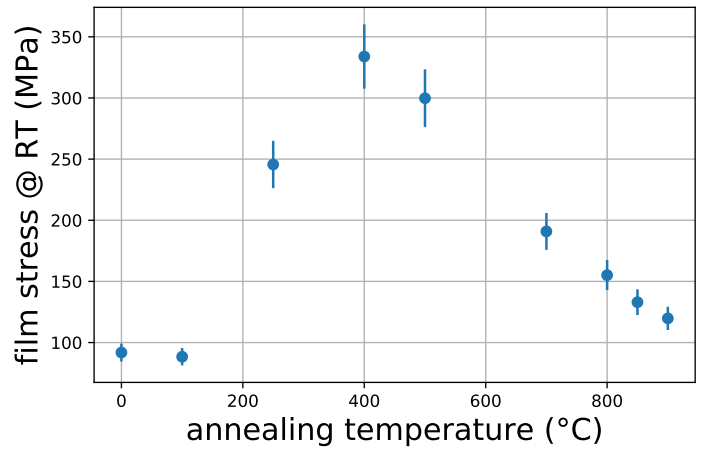

(b)

Figure 5.22: Experimental results for a GE124 cover glass covered with 360(20) nm of $\mathrm{SiO}_{x}$. The sample was annealed in nitrogen flow at increasing temperatures for $6 \mathrm{~h}$ at each temperature. (a) The transmission spectra of the sample after annealing at the stated temperature. (b) The film stress measured at room temperature after the corresponding annealing step. An entirely elastic deformation was assumed. However, for annealing at $800^{\circ} \mathrm{C}$ and above, viscous flow might have contributed to the measured curvature.

A similar behavior is observed in Fig. $5.22 \mathrm{~b}$ for the average film stress at room temperature, which I calculated by the Stoney Eq. (1.1) from the curvature difference with respect to the non-coated sample. After deposition, the film stress is tensile with a value of 92(7) MPa. Between 100 and $400{ }^{\circ} \mathrm{C}$ it increases (becomes more tensile) to a value of $330(30) \mathrm{MPa}$ and decreases for annealing at higher temperatures. For annealing at temperatures of $800^{\circ} \mathrm{C}$ and above, a contribution of viscous flow cannot be excluded.

These results demonstrate that at least two different processes happen during 
annealing of $\mathrm{SiO}_{x}$ films. The first process dominates at temperatures below $400{ }^{\circ} \mathrm{C}$ and the second process dominates above $400^{\circ} \mathrm{C}$. The underlying mechanisms will be discussed in Sec. 5.3.1,

\subsubsection{Stresses induced by laser ablation of silicon suboxide films}

\section{Backside ablation}

For the experiments described in Sec. 5.1, I structured $\mathrm{SiO}_{x}$ films via backside ablation with an ArF excimer laser, and I demonstrated in Ch. 3 that laser ablation induces a quasi-permanent stress inside the glass surface. Therefore, I studied if such a stress can also be observed in backside ablation. For this, I used three samples of GE124 cover glasses covered with approximately 310, 650 and $1400 \mathrm{~nm}$ thick $\mathrm{SiO}_{x}$ films. Before and after backside ablation of the films, I measured the curvatures on the non-coated side and calculated the integrated film stress.

Fig. 5.23 shows an example for the curvature profiles after film deposition and after removing the whole film by laser ablation with a homogenized laser spot of approximately quadratic cross section and an edge length of about $1.15 \mathrm{~mm}$. After deposition, the $650 \mathrm{~nm}$ thick film exhibits a tensile stress of about $90 \mathrm{MPa}$ and an integrated stress of $60(2) \mathrm{N} / \mathrm{m}$. One half of the sample surface (left in Fig. 5.23) was decoated with a fluence of about $500 \mathrm{~mJ} / \mathrm{cm}^{2}$, while the other half (right in Fig. 5.23 was decoated with a fluence of about $2 \mathrm{~J} / \mathrm{cm}^{2}$. In both cases, the curvature increased, but is still clearly below the zero line, that indicates the non-deformed state of the substrate. Thus, even after decoating, some tensile near-surface stress is left. The corresponding integrated stress is roughly about $20 \mathrm{~N} / \mathrm{m}$. Additionally, the curvature is smaller (more negative) for ablation with a higher fluence. If the stress would be the result of a thin residual film of $\mathrm{SiO}_{x}$ after ablation, one would expect that the curvature is larger (more positive) at the higher fluence because more material should have been ablated. Plus, for another sample with smaller film thickness, hence larger initial curvature, even no increase of the curvature after decoating at high fluence was observed. This shows, that the stress is not caused by residual $\mathrm{SiO}_{x}$ but is generated by the ablation process.

The curvature of the decoated samples can further be increased by annealing in air at $900{ }^{\circ} \mathrm{C}$ for tens of hours or by ultrasonic treatment in a $\mathrm{KOH}$ based detergent. I observed no curvature change for irradiation of a pristine (non-coated) substrate. Therefore, the generated stress is definitely caused by ablation of the $\mathrm{SiO}_{x}$.

\section{Front side ablation}

To observe the stress change in $\mathrm{SiO}_{x}$ films by excimer laser ablation in front side orientation, a GE124 cover glass was coated with a film of 428(9) nm thickness, and one half of the film was irradiated in front side orientation with a homogenized laser 


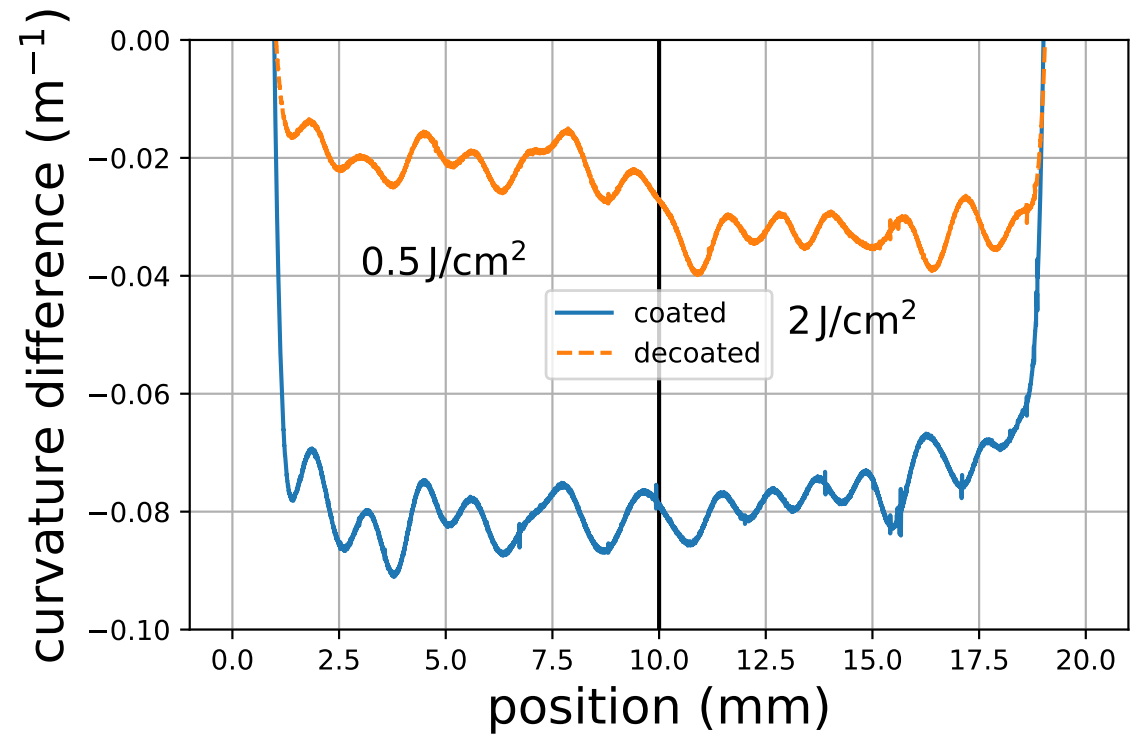

Figure 5.23: Curvature profiles of a sample with about $650 \mathrm{~nm}$ of $\mathrm{SiO}_{x}$ on a $\mathrm{GE} 124$ cover glass. The sample was coated and subsequently fully decoated by backside ablation at two different fluences. A negative curvature corresponds to a tensile film stress.

spot at a fluence of about $1.8 \mathrm{~J} / \mathrm{cm}^{2}$ and one pulse per position. Fig. 5.24 shows the result of the experiment. The average curvature is $-45.6(7) \cdot 10^{-3} \mathrm{~m}^{-1}$ after deposition, which corresponds to a tensile integrated stress of $43(2) \mathrm{N} / \mathrm{m}$. In the irradiated area, the curvature decreases to a value of about $-125 \cdot 10^{-3} \mathrm{~m}^{-1}$, which corresponds roughly to an integrated stress of $117 \mathrm{~N} / \mathrm{m}$ (if non-local mechanical effects are neglected). After total decoating of the film in backside orientation at $450 \mathrm{~mJ} / \mathrm{cm}^{2}$, no indication of the prior irradiation step is left (not shown). Thus, by the first irradiation step at $1.8 \mathrm{~J} / \mathrm{cm}^{2}$, an additional tensile stress has been generated inside the film or a compressively stressed layer has been removed. In Fig. 5.24b the transmission spectra of the sample after deposition and of the irradiated area are shown. By irradiation, the transmission decreased, although at the applied fluence, ablation of about $150 \mathrm{~nm}$ in depth is to be expected [84], which should lead to an overall increase of transmission.

In Fig. 5.22, a simultaneous decrease of the transmission and increase of integrated stress of an $\mathrm{SiO}_{x}$ film was demonstrated for annealing of the sample. To check if a similar process is activated by the irradiation, I first annealed a similar sample to the one of Fig. 5.24 at $900{ }^{\circ} \mathrm{C}$ in nitrogen flow for $4.5 \mathrm{~h}$ and subsequently irradiated it analogously. This time, an increase of the curvature (less negative) and correspondingly a decrease of the integrated stress (less positive) was observed after irradiation. Presumably, the annealing step already induced the processes responsible for the curvature change in Fig. 5.24. Thus, the above described generation of a tensile stress component by front side irradiation is presumably caused by short time annealing of the film. 


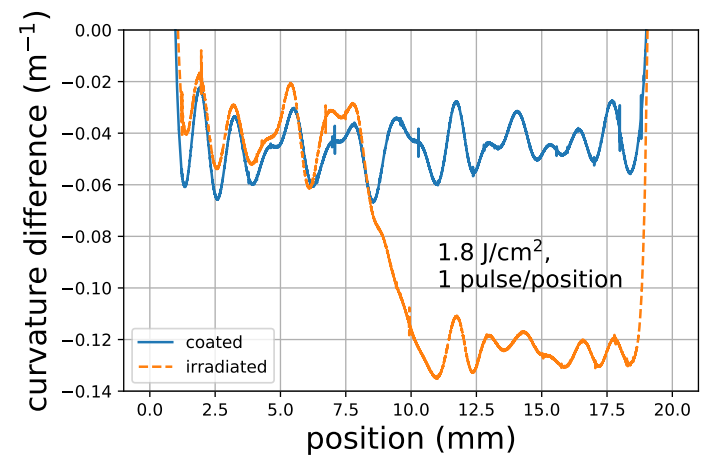

(a)

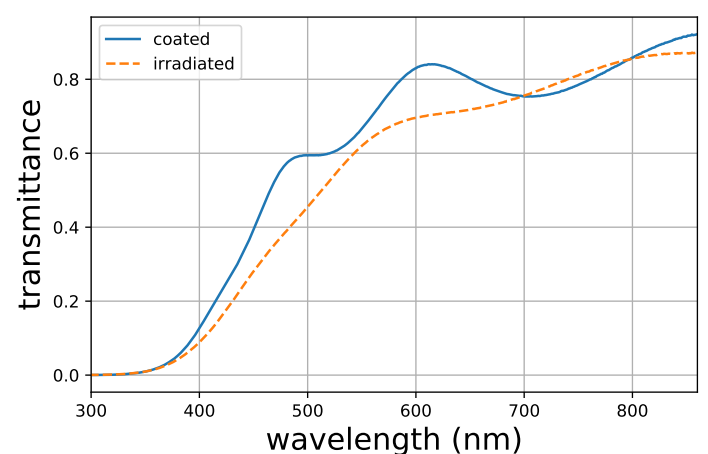

(b)

Figure 5.24: Experimental results for a GE124 cover glass covered with 428(9) nm of $\mathrm{SiO}_{x}$. After coating, one half of the coated side was irradiated in front side orientation with a homogenized laser spot at $1.8 \mathrm{~J} / \mathrm{cm}^{2}$ and one pulse per position. (a) The curvature profiles of the sample in direction across the boundary between pristine and irradiated film. (b) The transmission spectra of the sample before irradiation and inside the irradiated area.

\subsubsection{Oxidation kinetics}

It has been argued that the oxidation of $\mathrm{SiO}_{x}$ films proceeds by transversal propagation of a distinct interface between the oxide $\left(\mathrm{SiO}_{2}\right)$ and the film $\left(\mathrm{SiO}_{x}\right)[153,157$, 158]. However, a homogeneous oxidation over the film thickness was also reported for certain deposition conditions $[156]$. Therefore, I studied the oxidation of the $\mathrm{SiO}_{x}$ films used in this work in detail.

An example for the transmission and reflection of the coated side of a fused silica substrate coated with a $\mathrm{SiO}_{x}$ film after annealing in air is shown in Fig. 5.25. Here, the film thickness was 500(8) nm before annealing. Annealing was performed in air at a temperature of $1120(1){ }^{\circ} \mathrm{C}$ for a duration of $3 \mathrm{~h}$ and led to the evolution of interference peaks in the UV spectral region. A comparison with the transmission spectrum shows that the $\mathrm{SiO}_{x}$ film is still highly absorbing in the UV region. Thus, the evolved peaks are an indication of a transparent film on top of the $\mathrm{SiO}_{x}$ film. Assuming a bilayer system of a $\mathrm{SiO}_{2}$ film on a $\mathrm{SiO}$ film, I determined the film thicknesses to be 347 and $231 \mathrm{~nm}$, respectively, via a fit to the experimental reflectance spectrum. The existence of the interference peaks in the UV spectral region is a proof for a distinct interface during oxidation. I obtained similar results for oxidation in air at $900^{\circ} \mathrm{C}$, oxidation in flowing oxygen at 800,900 and $1060{ }^{\circ} \mathrm{C}$ and for oxidation in flowing nitrogen at $1060{ }^{\circ} \mathrm{C}$. The latter was presumably caused by traces of oxidizing species.

To further evaluate the oxidation kinetics of $\mathrm{SiO}_{x}$ films, I measured the oxide thickness in dependence on total annealing time for annealing in oxygen flow at a temperature of $920(20)^{\circ} \mathrm{C}$ for a sample of about $850 \mathrm{~nm}$ of $\mathrm{SiO}_{x}$ on a $1 \mathrm{~mm}$ thick substrate of Heraeus Suprasil. For comparison, I simultaneously annealed a silicon sample with a polished surface. 


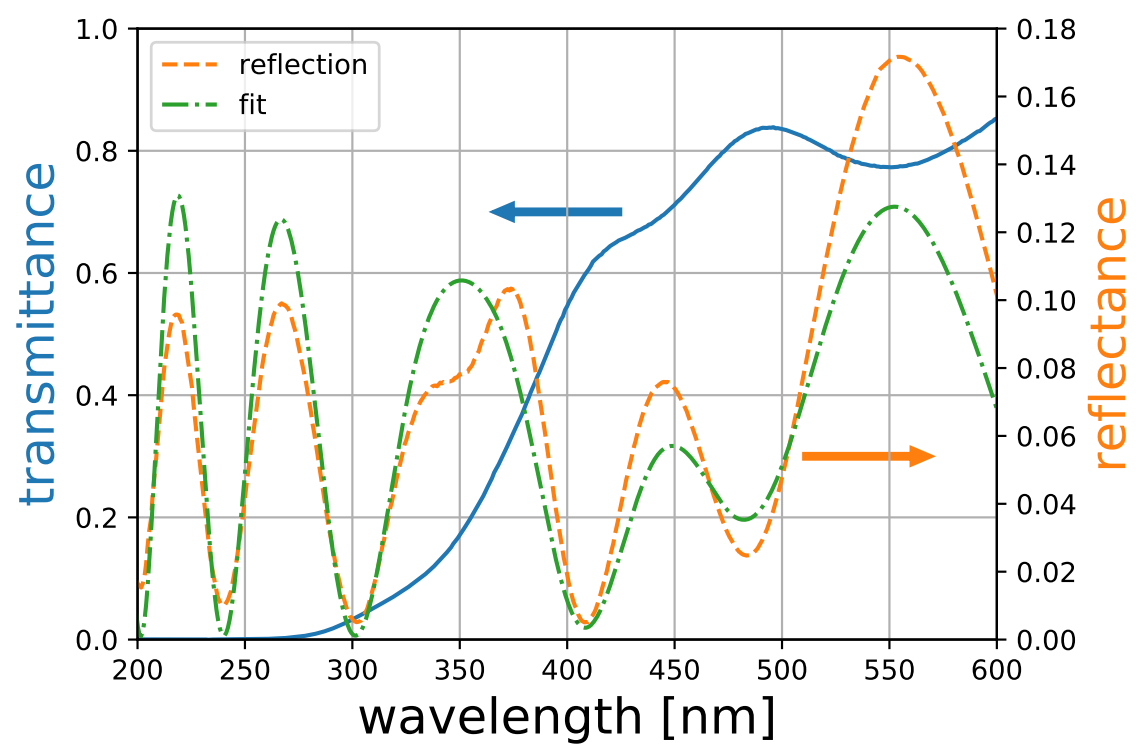

Figure 5.25: The transmittance and reflectance of a sample with 500(8) nm $\mathrm{SiO}_{x}$ on a $1 \mathrm{~mm}$ thick fused silica substrate after oxidation in air at $1120(1)^{\circ} \mathrm{C}$ for $3 \mathrm{~h}$. The reflectance was measured on the coated side of the sample. For determination of the thickness of the $\mathrm{SiO}_{2}$ and the $\mathrm{SiO}_{x}$ film, the reflectance spectrum has been fitted by variation of the corresponding layer thicknesses.

Fig. 5.26 shows the results of the experiment. Oxidation of the $\mathrm{SiO}_{x}$ film proceeds much faster than oxidation of the silicon sample. For a quantitative analysis, the data was fitted by the model of Deal and Grove [2]

$$
x(t)=\frac{A_{\mathrm{dg}}}{2}\left[\sqrt{1+\frac{4 B_{\mathrm{dg}}\left(t+t_{0}\right)}{A_{\mathrm{dg}}^{2}}}-1\right]
$$

for the oxide thickness $x$ in dependence on oxidation time $t$. The parameters $A_{\mathrm{dg}}$ and $B_{\mathrm{dg}}$ depend on reaction rates, the diffusion properties of the oxide and the oxidation state of the material before oxidation. The parameter $t_{0}$ corresponds to the initial oxide thickness and can be used to compensate for any oxidation behavior that is not covered by the model at small oxidation times. For oxidation of silicon in dry oxygen atmosphere, such a behavior has been intensively discussed [2, 148, 165 168]. Therefore, in order to not restrict the parameter $t_{0}$ by the fitting procedure, the first measurement point at time $t=0$ was ignored in the fitting routine.

Tab. 5.1 summarizes the fit results and compares them to the original data of Deal and Grove for oxidation of silicon at $920^{\circ} \mathrm{C}$ in a dry oxygen atmosphere. The results for the parameters $A_{\mathrm{dg}}$ and $B_{\mathrm{dg}}$ agree well between the silicon sample and the literature values. However, they differ by the parameter $t_{0}$. This difference corresponds to a difference in film thickness of roughly $10-20 \mathrm{~nm}$. Nevertheless, the overall good agreement for the oxidation of silicon excludes any major experimental errors. For the oxidation of the $\mathrm{SiO}_{x}$ film, the small values for the parameters $A_{\mathrm{dg}}$ and $t_{0}$ indicate a simple parabolic oxidation behavior $x(t)=\sqrt{B_{\mathrm{dg}} \cdot t}$ with $B_{\mathrm{dg}}$ being twice to third times as large than in the case of silicon. 


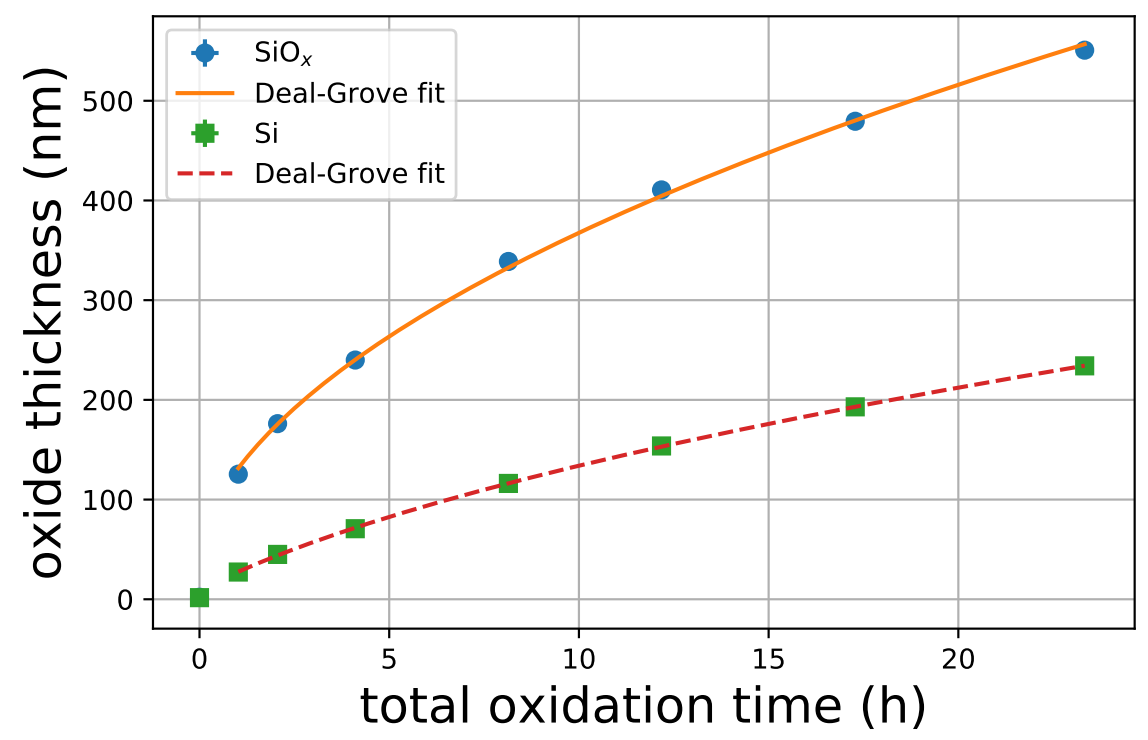

Figure 5.26: The oxide thickness as a function of the annealing time in oxygen atmosphere at $920(20)^{\circ} \mathrm{C}$ for a $\mathrm{SiO}_{x}$ film and a polished silicon sample. The results of both samples have been fitted by the Deal-Grove model (5.4) excluding the first measurement point at zero oxidation time.

Table 5.1: The results of a Deal-Grove fit for a $\mathrm{SiO}_{x}$ film and a silicon sample compared to the results of Deal and Grove (third line) from [2]. Oxidation was performed in oxygen flow at $920(20)^{\circ} \mathrm{C}$.

\begin{tabular}{|c|c|c|c|}
\hline sample & $A_{\mathrm{dg}}$ in $\mu \mathrm{m}$ & $B_{\mathrm{dg}}$ in $\mu \mathrm{m}^{2} / \mathrm{h}$ & $t_{0}$ in $h$ \\
\hline $\mathrm{SiO}_{x}$ film & $0.00(4)$ & $0.013(1)$ & $0.3(3)$ \\
silicon & $0.20(1)$ & $0.0043(1)$ & $0.44(8)$ \\
silicon[2] & 0.235 & 0.0049 & 1.4 \\
\hline
\end{tabular}




\subsubsection{Deformation of vitreous silica cover glasses during ox- idation at $900^{\circ} \mathrm{C}$}

To learn more about the stresses that occur during oxidation of $\mathrm{SiO}_{x}$ films, I annealed GE124 cover glasses covered with $\mathrm{SiO}_{x}$ films at $900^{\circ} \mathrm{C}$ in ambient air and in flowing oxygen and studied their curvature evolution by measurement at room temperature.

Fig. 5.27a shows the curvature evolution of a sample covered with 360(20) nm of $\mathrm{SiO}_{x}$ for annealing in flowing oxygen. After deposition, I pre-annealed the sample in flowing nitrogen to stabilize the film. Therefore, in Fig. 5.27a, the curvature starts at the value measured after annealing for $6 \mathrm{~h}$ at $900^{\circ} \mathrm{C}$. The curvature follows a square-root-like dependence until, at a total oxidation time of $60 \mathrm{~h}$, a value of about $0.23 \mathrm{~m}^{-1}$ is reached. For longer oxidation times, it relaxes to a value of about $0.15 \mathrm{~m}^{-1}$. From the transmission spectra of the sample (not shown), it can be deduced that oxidation was complete after $76 \mathrm{~h}$ of annealing. Hence, the breaking point in curvature evolution is reached when oxidation is complete.

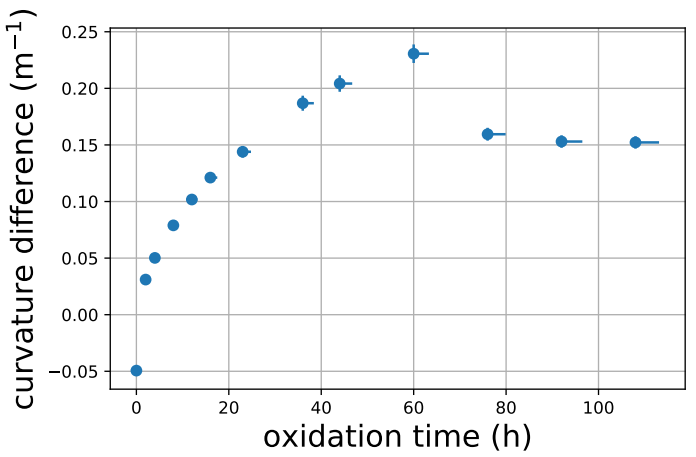

(a)

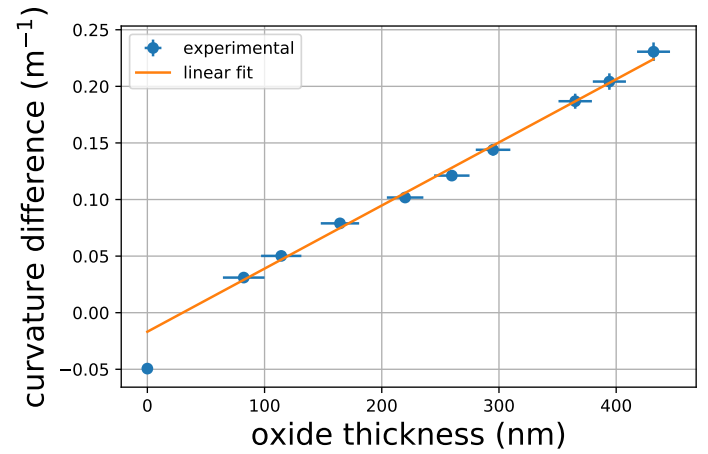

(b)

Figure 5.27: Experimental results for a GE124 cover glass covered with 360(20) nm of $\mathrm{SiO}_{x}$. After coating, the sample was annealed at $900{ }^{\circ} \mathrm{C}$ in nitrogen flow and subsequently in oxygen flow at the same temperature. Here, only the curvature evolution for annealing in oxygen is considered. In (a) the curvature difference with respect to the pristine substrate is plotted in dependence on the total oxidation time. In (b) the same curvature values (up to $60 \mathrm{~h}$ ) are plotted in dependence on the oxide thickness. The data points, excluding the one at zero thickness, have been fitted by a linear function.

By analysis of the transmission spectra and measurement of the film thickness after complete oxidation, I estimated the oxide thickness at every time step ${ }^{4}$. In Fig. $5.27 \mathrm{~b}$, the curvature is plotted in dependence on this estimate. The data is well described by a linear function if the initial curvature value is excluded, as can be seen by the linear fit in Fig. 5.27b.

Due to the many different effects (elasticity, delayed elasticity [118, pp. 45-46], viscosity, thermal expansion) that could play a role, the interpretation of the results

\footnotetext{
${ }^{4}$ The reflectometer for measuring the film thickness was bought after I performed these experiments.
} 
in Fig. 5.27 is difficult. However, presumably the decrease in curvature after oxidation is complete (Fig. 5.27a) can be attributed to relaxation of the stress in the grown oxide layer and an elastic deformation of the substrate, or to delayed elasticity of the substrate. Either way, the fact that the curvature does not reduce to zero could be caused by a non-instantaneous (viscous or delayed elastic) contribution to the deformation of the substrate.

To check for a non-instantaneous deformation of the substrate, I analogously oxidized similar samples for such a duration that a thin layer of $\mathrm{SiO}_{x}$ remained. Subsequently, I removed at least one half of the film via back side ablation and etched the samples in a $\mathrm{KOH}$ based detergent (Borer Chemie Deconex $15 \mathrm{PF}-\mathrm{x}$ ) to reduce the influence of the stresses induced by the ablation process (cf. Sec. 5.2.2). An example for oxidation of a $380(30) \mathrm{nm}$ thick $\mathrm{SiO}_{x}$ film for $30 \mathrm{~h}$ is shown in Fig. 5.28, After ablation of half of the film area, the curvature remains positive in the ablated region. Thus, the substrate deformation is composed of an elastic (instantaneous) and a non-instantaneous component.

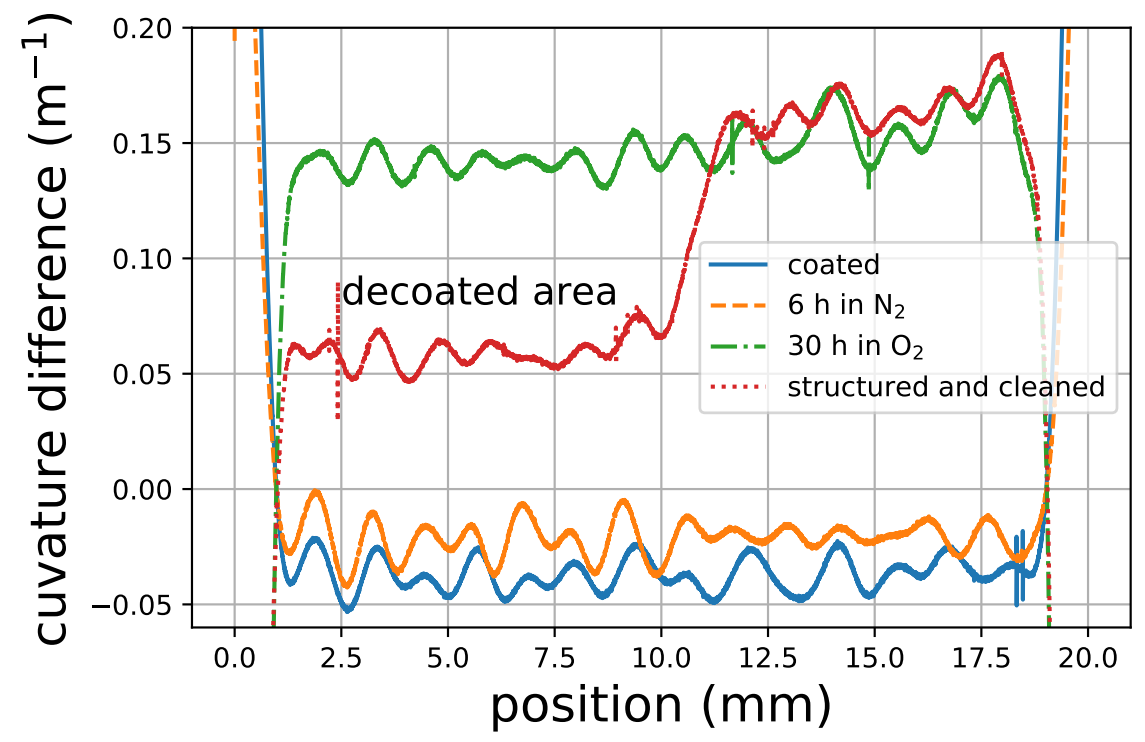

Figure 5.28: The curvature profiles of a GE124 cover glass coated with 380(30) nm of $\mathrm{SiO}_{x}$. After deposition, the sample was annealed in nitrogen and subsequently in oxygen for $30 \mathrm{~h}$ at $900^{\circ} \mathrm{C}$. Via backside ablation, the film was removed on one half of the sample surface and the sample cleaned in a $\mathrm{KOH}$ based detergent.

I measured a thickness of the grown $\mathrm{SiO}_{2}$ layer after the oxidation step of $439(3) \mathrm{nm}$. Assuming a thickness increase by oxidation of 1.3, a layer of about $10-70 \mathrm{~nm}$ of $\mathrm{SiO}_{x}$ remained. Neglecting the mechanical influence of this layer, an average compressive film stress at room temperature of roughly $-160 \mathrm{MPa}$ can be estimated for the $\mathrm{SiO}_{2}$ layer by the Stoney equation and the curvature change by film ablation. I achieved a similar estimate of roughly $-150 \mathrm{MPa}$ for the sample of Fig. 5.27 by considering the curvature change after complete oxidation of the film in Fig. 5.27a. In this case the film thickness after oxidation was 469(3) nm. The similarity between the curvature decrease in Fig. 5.27 a by annealing and the curvature decrease in Fig. 5.28 by laser ablation indicates that the curvature decrease in Fig. 
$5.27 \mathrm{a}$ is caused by relaxation of the stress inside the oxide layer. If the same thermal expansion coefficient for the $\mathrm{SiO}_{2}$ oxide layer and the $\mathrm{SiO}_{2}$ substrate is assumed, the results further indicate that oxidation of the $\mathrm{SiO}_{x}$ film causes a compressive stress inside the grown oxide layer that is smaller (higher absolute value) than -150 MPa.

\subsection{Discussion}

In Secs. 5.1 and 5.2, I presented results on the mostly viscous and viscoelastic deformation, respectively, of silica plates by continuous and structured films of $\mathrm{SiO}_{x}$ deposited on one side of the plates. For annealing at $1120^{\circ} \mathrm{C}$ in air and at $1060^{\circ} \mathrm{C}$ in flowing oxygen or nitrogen, a large deformation of $1 \mathrm{~mm}$ thick plates is observed, with amplitudes of up to more than $100 \mu \mathrm{m}$ and curvatures of up to more than $1 \mathrm{~m}^{-1}$. In Sec. 5.2, I presented additional results on the behavior of fused quartz cover glasses as substrates and on the behavior of $\mathrm{SiO}_{x}$ for annealing at temperatures below $920^{\circ} \mathrm{C}$. In the following these results are discussed.

\subsubsection{The origin of the film stress}

From the experimental results presented in Sec. 5.1, it is clear that the viscous deformation of the fused silica plates is related to an overall compressive stress generated by the deposited $\mathrm{SiO}_{x}$ film. More troubling is the question of the origin of the film stress and its temporal behavior. Generally, a film stress can be decomposed into an external contribution, by forces acting on the film-substrate system, a thermal contribution and an intrinsic contribution [4]. In the present case, the external contribution can be neglected.

Regarding the thermal contribution, in [169] the linear thermal expansion coefficient of $\mathrm{SiO}_{x}$ films with $x \approx 1$ deposited by PECVD was measured to be about $9 \cdot 10^{-6} \mathrm{~K}^{-1}$ in the range of 30 to $100^{\circ} \mathrm{C}$. This is more than a magnitude larger than the linear thermal expansion coefficient of fused silica of about $0.5 \cdot 10^{-6} \mathrm{~K}^{-1} 100$, Tab. 2.13]. Thus, at least for a not too large temperature increase, a compressive thermal stress contribution should exist in the $\mathrm{SiO}_{x}$ film. Thermal expansion is a 'bulk' property, and therefore the integrated stress should depend on the film thickness. However, annealing of samples with different film thickness (Fig. 5.9a) shows that at $1060(10)^{\circ} \mathrm{C}$ there is no significant dependence of the sample curvature on the film thickness. Therefore, no thermal contribution to the film stress is observed at this temperature and presumably also at $1120^{\circ} \mathrm{C}$. The same argument holds for potential stresses generated by the phase separation of the film: The stress should be generated inside the 'bulk' of the film, and therefore the integrated stress should depend on the film thickness.

It is tempting to explain the absence of stresses inside the $\mathrm{SiO}_{x}$ film, away from the interfaces, for annealing at $1060(10)^{\circ} \mathrm{C}$ by relaxation of the stress inside the $\mathrm{SiO}_{x}$ film. Unfortunately, to the best of my knowledge, the viscosity of silicon suboxide has not been measured, yet. For a simple estimate of the maximum possible viscosity, 
we note that after annealing at such temperatures the film is decomposed into silicon nanoclusters inside a $\mathrm{SiO}_{x}$ matrix with $x \lesssim 2$, and that the clusters are already to a large extent crystallized [170, 171]. Thus, one might argue that the film can be considered as a suspension of hard silicon spheres inside a Newtonian $\mathrm{SiO}_{2}$ liquid, and therefore its relative viscosity $\eta_{r}=\eta / \eta_{0}$ with respect to the viscosity of the liquid $\eta_{0}$ can be estimated by the Roscoe equation 172

$$
\eta_{r}=\left(1-c_{\mathrm{eff}}\right)^{-2.5},
$$

with an effective volume concentration $c_{\mathrm{eff}}=c_{\mathrm{Si}} / c_{p}$ [173] calculated from the volume concentration $c_{\mathrm{Si}}$ of the silicon clusters and some percolation threshold $c_{p}$. With a value of $c_{\mathrm{Si}}=0.31$ for an average stoichiometry of silicon monoxide $(x=1)$ and $c_{p}=0.38[174$ as the percolation threshold for transport of photoexcited carriers, a value of $\eta_{r}=69$ is obtained. From this value, the relaxation time of the $\mathrm{SiO}_{x}$ film can be estimated by use of Eq. (4.24) for the relaxation time of a thin film:

$$
\tau_{\mathrm{SiOx}} \approx 3 \cdot 69 \frac{\eta_{0}}{G_{0}} .
$$

The instantaneous shear modulus $G_{0}$ of the film is approximated by $G_{0} \approx 30 \mathrm{GPa}$ for fused silica (cf. Tab. A.1). For the viscosity $\eta_{0}$ of the $\mathrm{SiO}_{2}$ matrix, the viscosity of bulk fused silica might be inserted. Unfortunately, the viscosity of fused silica depends on the impurity concentration: For the substrate material of Heraeus Suprasil, I obtained a value of $1 \cdot 10^{12} \mathrm{~Pa} \cdot \mathrm{s}$ at $1060(10)^{\circ} \mathrm{C}$ in Sec. 5.1.3. However, for pure fused silica, a value of $3 \cdot 10^{14} \mathrm{~Pa} \cdot \mathrm{s}$ would be expected at a temperature of $1060{ }^{\circ} \mathrm{C}[100$, Eq. (12) on p. 14]. Applying this range of viscosity values in Eq. (5.6), a relaxation time $\tau_{\mathrm{SiOx}}$ of $1.9 \mathrm{~h}$ to $23 \mathrm{~d}$ is obtained. According to this simple estimation, stresses inside the $\mathrm{SiO}_{x}$ film would not rapidly decline. However, a size-dependent melting temperature of silicon nanocrystals was observed [175]. According to Fig. 3 in [175], at a temperature of $1060^{\circ} \mathrm{C}$, silicon nanocrystals up to a radius of about $7 \mathrm{~nm}$ are molten already. Therefore, the above assumption of a suspension of solid silicon nanocrystals is wrong, and a much lower viscosity should be expected. Plus, even if the nanocrystals do not melt at the application temperature, in [176] a rapid stress relaxation inside silicon nanocrystals was observed. From Fig. 3(b) of [176] a stress decay time (defined by a stress decrease of $1 \mathrm{GPa}$ ) of smaller $3 \mathrm{~s}$ can be estimated by extrapolation to a temperature of $1060^{\circ} \mathrm{C}$. These observations support the assumption of a rapid stress relaxation inside the decomposed $\mathrm{SiO}_{x}$ at temperatures of $1060^{\circ} \mathrm{C}$ and above, and could explain the fact that the film stress does not depend on the film thickness.

Because thermal expansion has been experimentally ruled out for causing the film stress, the only possibility left is an intrinsic stress. Further, the independence of the curvature on the film thickness, that is demonstrated in Fig. 5.9a, suggests that the stress is located at an interface. The most obvious explanation for such a stress is the oxidation of the $\mathrm{SiO}_{x}$ film to $\mathrm{SiO}_{2}$ [146, 154, 177, 178].

By reflection measurements, I found that oxidation proceeds from the surface (Sec. 5.2.3), leading to the formation of a $\mathrm{SiO}_{2} / \mathrm{SiO}_{x}$ bilayer system (Fig. 5.29). Additionally, I studied the oxidation kinetics at a temperature of $920(20)^{\circ} \mathrm{C}$ in flowing oxygen. A simple parabolic/diffusion limited behavior with $A_{\mathrm{dg}}=0$, with 
$A_{\mathrm{dg}}$ being the parameter $A$ in the Deal-Grove model [2], is observed. Diffusion limited oxidation was implied in many works on $\mathrm{SiO}_{x}$ films 146, 153, 154, 156 158. The value I obtained for the parabolic rate constant $B_{\mathrm{dg}}$ is twice to third as large than for oxidation of silicon (Tab. 5.1). This can be explained by $B_{\mathrm{dg}} \propto 1 / n_{1}[2]$ with $n_{1}$ being the number density of oxidant molecules incorporated into the oxide layer during oxidation. Thus, for $x \approx 1\left(\mathrm{SiO}_{x} \approx \mathrm{SiO}\right), n_{1}$ is half the value for oxidation of $\mathrm{SiO}_{x}$ compared to oxidation of silicon and therefore twice the value for $B_{\mathrm{dg}}$ would be expected. The picture is complicated by phase separation of the $\mathrm{SiO}_{x}$, that results in a geometrically different oxidation behavior, and the influence of the oxide stress on the diffusion of the oxidizing species [178].

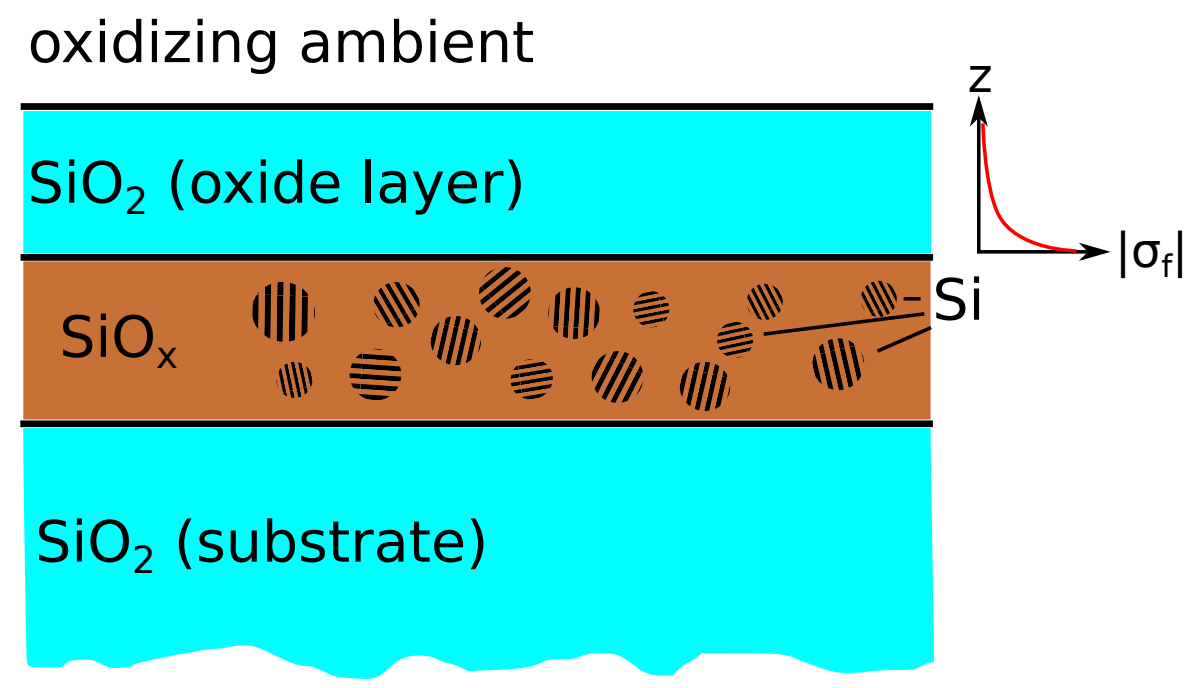

Figure 5.29: Scheme of the assumed cross section of a sample after annealing in oxidizing ambient for a specific duration. At elevated temperatures, the $\mathrm{SiO}_{x}$ film decomposes into silicon nanoclusters inside a matrix of high $(x \lesssim 2)$ oxygen concentration 170, 171]. Oxidation proceeds from the surface by forming a compressively stressed $\mathrm{SiO}_{2}$ layer. The growth stress $\sigma_{f}$ relaxes with time and therefore is most compressive at the $\mathrm{SiO}_{2} / \mathrm{SiO}_{x}$ interface.

For oxidation at about $900{ }^{\circ} \mathrm{C}$ in air, I estimated a maximum (minimum in absolute values) compressive oxide stress of about $-150 \mathrm{MPa}$ of the about $450 \mathrm{~nm}$ thick oxide layer (Sec. 5.2.4). And for annealing for $5 \mathrm{~h}$ in nitrogen flow at $1060(10)^{\circ} \mathrm{C}$, I obtained a value of roughly $-340 \mathrm{MPa}$ in Sec. 5.1.3 under the assumption that the film stress was generated by oxidation and substrate viscosity was constant. Due to relaxation, the obtained stress values are presumably bigger (smaller in absolute values) than the value of the instantaneous growth stress [179], which is the stress that is generated directly after oxidation at the $\mathrm{SiO}_{2} / \mathrm{SiO}_{x}$ interface. For the instantaneous growth stress for oxidation of silicon, values of $-460(50) \mathrm{MPa}$ and $-450 \mathrm{MPa}$ have been reported [150, 180]. In [146], a compressive stress of about $-500 \mathrm{MPa}$ was reported for deposition of $\mathrm{SiO}_{x}$ at small rate and high gas pressure, and subsequent exposure to the ambient atmosphere. Thus, the estimated values of $-150 \mathrm{MPa}$ and $-340 \mathrm{MPa}$ are of the same order of magnitude as would be expected from the literature.

For oxidation in air at $1120(1)^{\circ} \mathrm{C}$ and in nitrogen flow at $1060(10)^{\circ} \mathrm{C}$, I observed 
an approximately linear curvature evolution with time, which indicates, according to the viscoelastic Stoney Eq. (4.7), an approximately constant integrated stress if the substrate viscosity is assumed to be constant during oxidation. As I demonstrated for annealing in nitrogen (Sec.5.1.3, Fig. 5.8), such a behavior can also be reproduced by the viscoelastic Stoney equation with parabolic oxidation (4.19) if large values of the relaxation time $\tau_{f}$ of the $\mathrm{SiO}_{2}$ layer and of the initial growth stress $\sigma_{f}$ of this layer are assumed. Compared to the value of $\tau_{s}=\eta_{s} / G_{s} \approx 30 \mathrm{~s}$ (with $\eta_{s}=10^{12} \mathrm{~Pa} \cdot \mathrm{s}$ and $G_{s}=33 \mathrm{GPa}$ ) for the relaxation time of the substrate for annealing at $1060(10){ }^{\circ} \mathrm{C}$, the value of $\tau_{f}=7.9(5) \mathrm{h}$, which I obtained in Sec.5.1.3 by fitting Eq. (4.19), seems unrealistic large. However, the viscosity of fused silica is extremely dependent on the hydroxyl concentration [100, pp. 14-15], and for the relaxation of a thin film, the geometry needs to be considered, which is reflected by Eq. (4.24) [151, 152]. For annealing at $1050{ }^{\circ} \mathrm{C}$ of dry grown $\mathrm{SiO}_{2}$ on silicon, a relaxation time of $6-20 \mathrm{~h}$ was reported, which agreed reasonable well to an estimate by literature values of $5 \mathrm{~h}$ [179]. Therefore, a relaxation time of $\tau_{f}=7.9(5) \mathrm{h}$ is actually a realistic value. By the same fit of Eq. (4.19) in Sec. 5.1.3. I also obtained a value of $\sigma_{f}=-910(40) \mathrm{MPa}$ for the initial growth stress. Its absolute value is clearly larger than expected (see above), but still of the same order of magnitude. Therefore, considering the simple assumptions I made in the derivation of Eq. (4.19), the fit to the data for annealing in nitrogen flow at $1060(10){ }^{\circ} \mathrm{C}$ resulted in surprisingly realistic results.

However, Eq. 4.19) cannot explain the small difference in curvature after annealing for $5 \mathrm{~h}$ in oxygen flow to annealing in nitrogen flow at $1060(10)^{\circ} \mathrm{C}(\mathrm{Fig}$. $5.7 \mathrm{~b}$ ) when compared to the large difference in oxide thickness (Fig. 5.7a). Further, if the same value of $\sigma_{f}$ for the initial growth stress is assumed for annealing at $1120^{\circ} \mathrm{C}$, the value for $\tau_{f}$ is corrected to $\tau_{f}=1.7 \mathrm{~h}$ by the ratio of experimentally determined substrate viscosities at 1120 and $1060^{\circ} \mathrm{C}\left(2.2 \cdot 10^{11} \mathrm{~Pa} \cdot \mathrm{s}\right.$ and $1 \cdot 10^{12} \mathrm{~Pa} \cdot \mathrm{s}$, respectively), and the value for $B_{\mathrm{dg}}$ from the fit in Fig. $5.1 \mathrm{~b}$ is used, Eq. (4.19) overestimates the curvature values in Fig. $5.1 \mathrm{f}$ for oxidation at $1120^{\circ} \mathrm{C}$ by more than a magnitude. A tolerable description of the data in Fig. 5.1f can only be obtained by Eq. 4.19) if an initial growth stress is assumed that is more than a magnitude smaller than the value of $\sigma_{f}=-910(40) \mathrm{MPa}$. These two shortcomings of Eq. (4.19) indicate an oversimplification in the derivation. In [179], it was argued for the oxidation of silicon that the relaxation of the growth stress can be separated into a fast relaxation to an intermediate level and a slow relaxation to the fully relaxed state, where the slow relaxation process is supposed to correspond to the linear viscous flow of bulk fused silica. The fast relaxation process is not considered in Eq. (4.19). But, for oxidation of $\mathrm{SiO}_{x}$ at $900{ }^{\circ} \mathrm{C}$, I observed a fast relaxation of the film stress (Fig. $5.27 \mathrm{a}$ that could be a hint to a two step relaxation process. Further, in contrast to oxidation of a flat silicon surface, oxidation of phase-separated $\mathrm{SiO}_{x}$ involves the oxidation of approximately spherical silicon nanoparticles, which leads to a profoundly different stress distribution inside the material [178], which was not considered in the derivation of Eq. 4.19). At last, I assumed a very simple viscoelastic behavior in the derivations in Sec. 4.1. I assumed a Maxwell element, which results in only one relaxation time, for the deviatoric relaxation and did not consider delayed elasticity, dilatational relaxation and structural relaxation during annealing (cf. [118]).

As structural relaxation (cf. [118, Part 2]) can have a large influence on the viscosity (cf. [134, Sec. 6.7]), according to the viscoelastic Stoney Eq. (4.7), it can 
also influence the curvature evolution of the samples. It is convenient to describe the thermal history of a glass, and therefore in some respects its structure, by the fictive temperature $T_{f}$, which states the temperature at which a glass needs to be quenched to reach specific properties in the solid state (cf. [118, Ch. 9]). The substrates I used have been received with an unknown fictive temperature $T_{f 0}$. During annealing at $T_{1}=1060{ }^{\circ} \mathrm{C}$ or $T_{2}=1120^{\circ} \mathrm{C}$, the fictive temperature $T_{f}$ must have changed towards $T_{f 1,2}=T_{1,2}\left(T_{1,2}\right.$ means $T_{1}$ or $\left.T_{2}\right)$. Simultaneously, the viscosity $\eta\left(T_{1,2}, T_{f}\right)$, which depends on current temperature $T_{1,2}$ and fictive temperature $T_{f}$ (cf. [118, Sec. 11]), presumably changed as well from $\eta\left(T_{1,2}, T_{f 0}\right)$ towards $\eta\left(T_{1,2}, T_{f 1,2}\right)=\eta\left(T_{1,2}, T_{1,2}\right)$ (cf. [134, Sec. 6.7]). The time scale on which this change in fictive temperature happened can be roughly estimated by the structural relaxation time $\tau_{p}$, which in general is related to the fictive temperature as well (cf. [118, Ch. 11]). According to the data of [181] presented in 118, p. 143], for $T_{1,2} \approx T_{f 0}$,

$$
\tau_{p}=a \cdot \tau_{s}
$$

with $a$ being a dimensionless value in the range of 4 to 20 and $\tau_{s}=\eta / G_{0}$ being the Maxwell relaxation time, which depends on viscosity $\eta$ and instantaneous shear modulus $G_{0}$. If the obtained viscosity values $\eta\left(T_{1}\right)=1 \cdot 10^{12} \mathrm{~Pa} \cdot \mathrm{s}$ (Sec. 5.1.1) and $\eta\left(T_{2}\right)=2.2 \cdot 10^{11} \mathrm{~Pa} \cdot \mathrm{s}$ (Sec. 5.1.3) and $G_{0}=33 \mathrm{GPa}$ (Tab. A.1) are used in Eq. (5.7), structural relaxation times of $\tau_{p}=27 \mathrm{~s}$ to $\tau_{p}=10 \mathrm{~min}$ are obtained. However, in this estimate for the structural relaxation time $\tau_{p}$, the change in viscosity $\eta$ during structural relaxation was not considered and $T_{1,2} \approx T_{f 0}$ was assumed. Another estimation for the structural relaxation time can be obtained from measurements of the fictive temperature of a type-III vitreous silica, like Heraeus suprasil [98] I used in this thesis, via small angle x-ray scattering. In [182], the fictive temperature was measured during temperature cycles and isothermal annealing. It was found that, in the range of measurement uncertainty, the structural relaxation time is independent on the fictive temperature and can be described by

$$
\tau_{p}(T)=a_{1} \exp \left(a_{2} / T\right)
$$

with $a_{1}=10^{-12.9} \mathrm{~s}$ and $a_{2} \approx 48.5 \cdot 10^{3} \mathrm{~K}$ for the first heating curve of an asreceived sample. Eq. (5.8) leads to structural relaxation times of $\tau_{p}\left(T_{1}\right)=13 \mathrm{~min}$ and $\tau_{p}\left(T_{2}\right)=3 \mathrm{~min}$, which are similar to the above estimated values. These values for the structural relaxation time are a strong indication that in my experiments for annealing at $T_{1}$ and $T_{2}$, structural relaxation happened during the first annealing step and did not affect the viscosity on subsequent annealing steps. This is in agreement with my observations of a linear curvature decrease after complete oxidation (Figs. 5.1f and 5.7b), which indicates a constant viscosity, and justifies my assumption of a constant viscosity already during oxidation. However, for a better control of viscosity during the viscous deformation, the substrates should be stabilized, i. e. the fictive temperature set to the final value by annealing at the corresponding temperature, before deposition of the $\mathrm{SiO}_{x}$ film.

In principle, delayed elasticity (cf. [118, Part 1]), a non-instantaneous but recoverable strain, also contributed to the sample deformation. However, the delayed elastic strain is of similar value as the instantaneous elastic strain (cf. [118, Sec. $4.2]$ ), and the values of the instantaneous elastic strain are negligible small. This can be seen from the unrealistically large values of the hypothetical film stress I 
obtained by describing the sample deformation by an elastic model in Secs. 5.1.1 and 5.1.2. Thus, in my experiments, the influence of delayed elasticity is negligible small as well.

Although no contribution of thermal expansion, phase separation or crystallization of the silicon nanoparticles is observed at $1060^{\circ} \mathrm{C}$, these processes presumably contribute at lower temperatures. Indeed, in Fig. 5.22b, it is demonstrated that during annealing of a $\mathrm{SiO}_{x}$ film at increasing temperature, the film stress at room temperature varies. Although the absolute value of the stress could be a superposition of intrinsic and thermal contribution, the stress variation shows that due to annealing, the material properties are altered. This interpretation is verified by the simultaneous change of the transmission spectra in Fig. 5.22a. A similiar behavior as in Fig. 5.22 was observed in [183] for $\mathrm{SiO}_{x}$ films of different oxygen content deposited by magnetron sputtering. The transmission of the films increased for annealing up to a temperature of $400-500^{\circ} \mathrm{C}$ and decreased for annealing at higher temperatures. The film thickness exhibited a similiar behavior, which agrees well with the stress-temperature curve of Fig. 5.22b. Please note that according to [151], counterintuitively, the film thickness increases if the film stress becomes more tensile. In [183], the behavior below $400^{\circ} \mathrm{C}$ is explained by stress relaxation and healing effects inside the freshly deposited film. In contrast, the behavior above $400{ }^{\circ} \mathrm{C}$ to about $800^{\circ} \mathrm{C}$ is explained by the phase separation inside the $\mathrm{SiO}_{x}$ material. Therefore, my data indicate that the phase separation leads to a change in thermal expansion or even to an intrinsic film stress at temperatures of $400{ }^{\circ} \mathrm{C}$ and above. I found that front side ablation has a similar effect on the film stress at room temperature and on the transmission of the film as annealing. Indeed, in [184] it was reported that $\mathrm{KrF}$ excimer irradiation $(248 \mathrm{~nm}, 20 \mathrm{~ns})$ of $\mathrm{SiO}_{x}$ films causes the phase separation as well.

In Sec. 5.2.2, I demonstrated that backside ablation of a $\mathrm{SiO}_{x}$ on a fused silica substrate leads to a tensile stress inside the uncovered substrate surface. Further, the magnitude of the generated integrated stress increases with increasing fluence. It is known that for ablation above the fluence threshold for film removal, at about $300 \mathrm{~mJ} / \mathrm{cm}^{2}$, some material of the underlying surface is removed as well [87, 88. In [88], this is explained by plasma mediated etching for fluences above the plasma ignition threshold, which lies roughly at $1 \mathrm{~J} / \mathrm{cm}^{2}$. However, even for fluences below this threshold, ablation of the fused silica substrate can be observed [87]. In light of the here measured stress generation at the surface and the stress generation by the TGM discussed for irradiation of Schott D263M in Sec. 3.2.1, it seems plausible that the ablation for fluences below the plasma ignition threshold is caused by thermal loading. In [68], a stressed surface layer with a thickness of about $275 \mathrm{~nm}$ and a stress of about $80 \mathrm{MPa}$ was reported for direct ablation of fused silica with an $\mathrm{F}_{2}$ laser. These values lead to an integrated stress of $22 \mathrm{~N} / \mathrm{m}$, which is quite close to the value of $\approx 20 \mathrm{~N} / \mathrm{m}$ I estimated in Sec. 5.2.2 from my experiments. The good agreement is another argument for a thermal stress generated via backside ablation and additionally gives a rough estimate for the thickness of the laser affected zone of roughly $250 \mathrm{~nm}$. Nevertheless, the stresses generated by patterning of the $\mathrm{SiO}_{x}$ presumably do not contribute to the curvature evolution of the samples at $1060(10){ }^{\circ} \mathrm{C}$ and above because of fast stress relaxation. 
In summary, my results indicate that at $1060^{\circ} \mathrm{C}$, and presumably at $1120^{\circ} \mathrm{C}$ as well, the film stress is located at an interface. The absence of a stress in the 'bulk' of the film could be explained by a fast relaxation of stresses inside the $\mathrm{SiO}_{x}$. Presumably, the film stress is generated by oxidation, which proceeds from the surface by diffusion of oxidizing species and causes a compressive stress on the order of a few $-100 \mathrm{MPa}$ inside the freshly grown $\mathrm{SiO}_{2}$. As oxidation proceeds, the stress inside the older $\mathrm{SiO}_{2}$ material relaxes. Surprisingly, an approximately constant integrated film stress is observed, which could be related to a complex coupling of oxidation and growth stress inside the grown $\mathrm{SiO}_{2}$ layer. For a better understanding, further studies are necessary.

\subsubsection{Deformation and gravity}

Basically, the qualitative viscous deformation of the $1 \mathrm{~mm}$ thick fused silica plates studied in Sec. 5.1 is similar to the one for purely elastic behavior of film and substrate. This is demonstrated by comparison with the results of linear elastic simulations in Figs. 5.3, 5.4, 5.6, C.2 and C.4 for macroscopically (lateral dimension > substrate thickness) structured films. And even for the case of a microscopically (lateral dimension < substrate thickness) line structured film, the qualitative behavior is similar as expected for the elastic case, which is experimentally demonstrated in Fig. 5.18. The similarity between viscous and elastic deformation can be understood in light of the correspondence principle [40, Sec. 9.3.3] or quasi-elastic approach [118, pp. 295-298] for obtaining an approximate solution for a viscoelastic problem from the elastic solution by replacing an elastic modulus with a viscoelastic modulus. Because in linear elasticity the uniaxial strain $\epsilon=\sigma / E$ is given as the ratio of stress $\sigma$ to Young's modulus $E$, from a mathematical point of view, it does not matter for the resulting deformation if the value of the Young's modulus or the value of the stress is changed. In the correspondence principle or the quasi-elastic approach, the Young's modulus is changed, which makes sense from a physical point of view, but in the simulations in sections 5.1.1 and 5.1.2, I just applied an unrealistically large value of stress, because it was the more convenient approach.

Only at more precise observation, differences between the elastic deformation (without gravity) and the measured deformation can be observed. One of these differences is the non-uniformity of the curvature profiles, which becomes more pronounced with increasing annealing time. For annealing in horizontal orientation of samples with continuous or macroscopically structured films, this additional deformation appears in both measurement directions. Therefore, it is presumed to be isotropic. In contrast, for microscopically line structured films, a concave curvature profile develops in direction along the lines and a convex curvature profile develops in direction across the lines (Figs. 5.13 and 5.19).

This behavior fits qualitatively well to the simulated curvature profiles of Sec. 4.2 , which are generated by gravity. In case of a macroscopic film structure, the overall displacement of the sample is isotropic so that a round plate-like substrate contacts the underlay at the lower rim. In the simulation in Sec. 4.2.1, such a set of boundary conditions indeed leads to the concave shape of the curvature profile observed in the 
experiment. This is also demonstrated by the direct comparison of the measured profiles for a structured film with the results of a linear elastic deformation in Fig. 5.4. In case of a microscopically line structured film, however, an overall anisotropic displacement is observed so that the number of contact points to the underlay is ideally reduced to two. Such a set of boundary conditions is approximated in the simulation in Sec. 4.2.2. By this simulation, a convex shape of the curvature profiles across the lines and a roughly uniform curvature along the lines are predicted. If the actual set of boundary conditions is an intermediate state between bearing at the whole rim and bearing at two points, maybe a concave shape develops in direction along the lines, like it is observed in the experiments (Figs. 5.13a and 5.19a).

Interestingly, for a macroscopically structured film and vertical orientation during annealing, a concave shaped curvature profile can only be observed in direction parallel to the underlay (Figs. 5.5d and C.3d). Unfortunately, in the experiment, the boundary conditions were badly defined. Therefore, it was not possible to estimate the expected deformation of the sample. Further, it should be noted that in case of an elastic substrate deformation, a non-uniform curvature is expected for large displacements [1]. I cannot exclude that this effect also contributes to the observed non-uniformity of the curvature profiles. However, at this point, I also do not see the necessity to include such an effect into the explanation of the experimental data.

So far, I argued that the form of the curvature profiles is influenced by gravitational sag. Further evidence for the influence of gravity can be found by considering the values of the average curvatures. For annealing at $1120^{\circ} \mathrm{C}$ in horizontal sample orientation, the average curvature increases during oxidation and it decreases after oxidation is complete (Figs. 5.1f and 5.2e). Again, the decrease of the curvature can be qualitatively explained by the simulations of Sec. 4.2. Please note that also the increase in curvature in direction across the lines, predicted by the simulation in Fig. 4.10b, can be observed in the experiment for small line periods in Fig. 5.11b. If gravity is responsible for the decrease of curvature, there should be a different behavior in the case of vertical orientation. Indeed, for the samples annealed in vertical orientation (Figs. 5.5e and C.3e), it can be observed that the curvature decreases in one direction and increases in the other direction after oxidation is complete.

A quantitative analysis further confirms the gravitational influence. From Fig. 5.1f, it can be seen that for a continuous film after annealing for $5 \mathrm{~h}$ at $1120(1)^{\circ} \mathrm{C}$ in air, a curvature of about $0.8 \mathrm{~m}^{-1}$ is reached. If half of the film is removed in a macroscopic pattern, half the value should be reached. Indeed, in Figs. $5.2 \mathrm{e}$ and C.1e, after $5 \mathrm{~h}$, a curvature of approximately $0.4 \mathrm{~m}^{-1}$ is observed. Thus, presumably viscosity is similar for all three experiments, and therefore the rate of curvature decrease after complete oxidation should be roughly the same for all three samples if geometrical influences are neglected. Indeed, for the samples of Figs. 5.1f, 5.2e and C.1e, a curvature decrease of $-0.019,-0.014$ and $-0.017(\mathrm{~m} \cdot \mathrm{h})^{-1}$, respectively, is measured by linear fits to the data. Although the films are differently patterned, these values agree well. This is another argument for the influence of gravity.

Although there is convincing evidence on the major influence of gravity on the sample deformation, there is some discrepancy considering the quantitative curvature evolution in the case of vertical orientation. In Fig. 5.5e, for annealing in 
vertical orientation of a sample with half of the film removed, after $5 \mathrm{~h}$ of annealing, a curvature of about $0.8 \mathrm{~m}^{-1}$ is reached. Surprisingly, this is approximately double the value for annealing of a similar sample in horizontal orientation (cf. 5.2e). This difference cannot be explained by the absence of the influence of gravity in vertical orientation because from the above paragraph, for $5 \mathrm{~h}$ of annealing, only a curvature decrease of $0.075 \mathrm{~m}^{-1}$ is expected. Because of the badly defined boundary conditions in vertical annealing, improved experiments are necessary to understand this discrepancy.

In summary, gravity seems to have a large effect on the samples in that it influences the average curvature values and leads to a non-uniformity of the curvature profiles. However, for a more precise analysis of the gravitational influence, a better control of the mechanical boundary conditions would be necessary. Precisely defined boundary conditions are especially important if an antibiaxial plane-stress component is generated by the film structure or the sample is not oriented horizontally.

\subsubsection{Line structured films}

For the same sample system $\left(\mathrm{SiO}_{x}\right.$ on Suprasil) studied here and annealing in air at 1000 and $1100^{\circ} \mathrm{C}$, an overall anisotropic deformation in the case of line structured films is described in [90]. This anisotropy is also observed in section 5.1.4 for annealing in air and in nitrogen flow at 1120 and $1060^{\circ} \mathrm{C}$, respectively. It is demonstrated that the curvature ratio $k_{s} / k_{p}$ increases with increasing line period at constant height. This result is in agreement with the case of elastic deformation, for which it was shown that the aspect ratio of the lines determines the resulting curvature ratio $3,19,20]$.

For annealing in nitrogen flow at $1060^{\circ} \mathrm{C}$, the evolution of the curvature ratio with annealing time is plotted in Fig. 5.17. As is proposed by the viscoelastic version of the theory of Wikström et al. I derived in Sec. 4.1.3, the curvature ratio shows an asymptotic behavior. According to the derived Eq. (4.44), the curvature ratio should decrease with time (Fig. 4.6a). While this is the case for the samples with 20 and $200 \mu \mathrm{m}$ line period, the inverse behavior is observed for the sample with $100 \mu \mathrm{m}$ line period (Fig. 5.17). Another discrepancy can be found when the time scale, on which the curvature ratio changes, is considered. In the experiment (Fig. 5.17), it is on the order of tens of hours, which corresponds to more than $10^{3} \cdot \tau_{s}$, if the estimated value of $\eta_{s}=1 \cdot 10^{12} \mathrm{~Pa} \cdot \mathrm{s}$ for the substrate viscosity (Sec. 5.1.3) is used for calculation of the relaxation time $\tau_{s}$. However, the theory predicts a time scale on the order of a few tens of $\tau_{s}$, as can be seen from Fig. 4.6a. These differences between experiment and theory could indicate a shortcoming of the theory with regard to the viscoelastic behavior of the substrate.

In Fig. 5.18, the curvature ratio after annealing for $34 \mathrm{~h}$ is compared to the expected curvature ratio in the elastic case and the expected final curvature ratio for the derived viscoelastic equation (4.45). The experimental curvature ratio does not agree with one of those theories, but a similar behavior is observed. In addition to the above mentioned shortcomings of the theory, ignorance of the right parameter 
values for the theoretical calculation could be one reason for the disagreement, as was already discussed in section 5.1.4. Another reason could be the distribution of the stress inside the cross section of the line segments. In the theory of Wikström et al., thermal stresses, and therefore a roughly homogeneous distribution of the stress before film patterning in direction normal to the surface, are assumed [3]. The same holds for other studies [19, 20, 185] of similar setups. However, in the current case, the stress is presumably caused by oxidation (cf. section 5.3.1), and therefore the prestressed area inside the line cross section might be thin. Plus, in case of fast oxidation, the position of this layer will change with time. Preliminary results of a linear elastic FE simulation indicate that these two aspects can have a large effect on the volume averaged stresses inside the line structure, which in turn has an effect on the curvature ratio. The simulations suggest that the curvature ratio will be overestimated by the theory of Wikström et al. if only the top part of the line is prestressed in the experiment. This result might explain why in Fig. 5.18 the experimental observed curvature ratio is overestimated both by the elastic and the linear viscoelastic estimate. Further, the simulations suggest that the curvature ratio increases if a thin stripe of prestressed material is moved from the top of the line segment to the bottom at the interface to the substrate. This effect could be another contribution to the evolution of the curvature ratios with time in Fig. 5.12 for annealing in air at $1120^{\circ} \mathrm{C}$. In case of oxidation of the line structured $\mathrm{SiO}_{x}$ films, the height of the lines increases significantly (cf. Fig. 5.14). This effect could also contribute to the curvature ratio by increasing the aspect ratio of the lines.

Further, there is the gravitational influence, as was already discussed in the preceding section. As is shown in Fig. $4.10 \mathrm{~b}$ by a linear viscoelastic simulation, gravitational deformation presumably leads to a decrease of curvature in direction along the lines and to an increase of curvature in direction across the lines. Therefore, the curvature ratio will be increased by gravity. This effect will be especially pronounced after oxidation is complete and could be another reason for the increase of curvature ratio with time in Fig. 5.12 for annealing in air. Additionally, the non-uniformity of the curvature profiles, that is induced by gravity, complicates the interpretation of the average curvature values.

Besides the curvature evolution of the substrate, I found that for oxidation in air at $1120^{\circ} \mathrm{C}$, the cross section of the line structures is changed. Because of oxidation, a height increase would be expected. However, in Fig. 5.14 also a rounding of the edges and a convex deformation of the decoated region can be observed. By comparison to the results of a linear elastic FE simulation, I concluded that the rounding and the deformation of the uncovered surface are caused by the stress in the lines and the corresponding deformation of the substrate surface. The deformation of the substrate surface can possibly be verified by only partial oxidation of the lines and subsequent removal by backside ablation. In [186], a deformation of the underlying fused silica substrate surface was observed for laser annealing of the $\mathrm{SiO}_{x}$ on top. This could be a similar stress effect.

In summary, for annealing in nitrogen flow, a similar behavior of the final curvature ratio on the aspect ratio as predicted by the linear elastic and the linear viscoelastic theory is observed. This shows that the deformation can be understood by the stress distribution inside the cross section of the lines and is a step towards 
the quantitative description of the sample system. However, for a better description, many different effects, like the stress distribution due to oxidation, the influence of gravity and the precise viscoelastic behavior of the substrate material need to be considered or their influence need to diminished by improved experiments.

\subsubsection{Application of the viscous deformation}

The patent 90 describes a process for forming of plates by deposition of a film of a substoichiometric oxide, generation of a compressive film stress by oxidation of the film and plastic or viscous deformation of the plate during annealing. At this point, I would like to discuss the difficulties in such a process for deformation of silicate glass plates and the steps to overcome them.

In analogy to the experimental part of this work, I assume that the problem consists of a plate of a silicate glass that is covered by a thin film. The film shall exhibit a certain stress at a given process temperature. Let us further assume that the process is fully deterministic, and all parameters are known. Then, from a certain film structure, it is possible to calculate the expected deformation. The other way round, namely calculating the necessary film pattern to reach a given deformation, can have more than one solution [38]. One solution might be found by numerically iterating the film pattern or by fragmentation of the desired deformation into a combination of an appropriate set of basis deformations, for each of which the necessary plane-stress field is known. The latter method has been successfully used in the elastic case [34, 36, 41]. The space of accessible deformations will be determined by the properties of the film and the patterning method. For example, in correspondence to the elastic deformation, if also antibiaxial plane stress components can be generated, the space of accessible deformations is larger than in case of only equibiaxial plane stress components [38].

In practice, gravity can influence the deformation, as was observed in the experimental part of this work. This influence could be reduced by different approaches or it could be included in the process. Presumably, increasing the processing viscosity at constant integrated film stress is not an option because deformation by film stress and gravity scale the same way with viscosity. However, one approach to reduce the relative gravitational influence is to increase the integrated film stress. For the case of the system studied in this work, this might be achieved by lowering the annealing temperature to increase the viscosity of the film. Please also note that the values for integrated stress of the film of $N_{1} \approx-13 \mathrm{~N} / \mathrm{m}$ and $N_{2} \approx-21 \mathrm{~N} / \mathrm{m} \mathrm{I}$ estimated in Secs. 5.1.1 and 5.1.3, respectively, are rather moderate. This can be seen by calculation of the average film stress via $\sigma_{f}=N / t_{f}$ with a film thickness of $t_{f} \approx 500 \mathrm{~nm}$. Thus, average stresses of $\sigma_{1} \approx-26 \mathrm{MPa}$ for $N_{1}$ and $\sigma_{2} \approx-42 \mathrm{MPa}$ for $N_{2}$ are obtained, of which the absolute values would be considered to be small in coating community [5]. Presumably, by a different film material, absolute stress values in the range of a few $100 \mathrm{MPa}$ could be obtained. Therefore, at the same film thickness, the relative influence of gravity could be reduced by one magnitude. The challenge is to identify a film material that fulfills all requirements for application. 
Another approach to reduce the influence of gravity, is to choose a proper sample orientation. A simple embodiment was already tested in this work by vertical orientation. However, finding appropriate boundary conditions could be difficult, and the residual gravitational deformation would be more difficult to predict, if necessary, than in the case of horizontal orientation. A sample holder for vertical orientation without elastic deformation of thin wafers is described in [28]. It would be a considerable task to design a similar holder for high temperature processes. To reduce the gravitational influence during gas bearing slumping (see below) of Schott D263 glass, it was suggested to glue tungsten wires to the sample and to hang it vertically oriented inside the oven [94]. This could indeed lead to a simple set of boundary conditions.

A third approach to reduce the influence of gravity could be to choose a soft underlay in horizontal sample orientation that, like a mattress, supports even the deformed plate at every point of its lower side. The success of such an approach is reported in 9 for avoidance of the elastic deformation of a flat mirror substrate during form measurement. The authors used a polymer foam as underlay material. Unfortunately, to find a suitable material that is stable at high temperatures and copes with the large deformations in the viscous case is a challenge. I performed experiments with fused silica wool as the underlay material with little success. Another material for the underlay could be a glass with a lower viscosity than the one of the sample. In that case, elemental diffusion and adhesion would presumably cause problems. Floating the sample on a constant gas stream is in my opponion also no adequate solution. In [39, 94], gas bearing slumping, in which the sample floats on a nitrogen stream, was studied with the result of considerable unintentional deformation of the sample. In gas bearing slumping there is still a mold applied. In our moldless process the unintentional deformations could become even worse.

If the gravitational influence cannot be reduced to a tolerable level, it needs to be included in the process. Unfortunately, the gravitational deformation depends on the boundary conditions and they, in turn, might depend on the deformation of the sample, which complicates prediction of the deformation a lot if an anisotropic curvature change, like in the case of a line structure, is generated. To avoid the connection between deformation and boundary conditions, a three point bearing should be used. However, this will also lead to a complex gravitational deformation. It could be difficult or even impossible (depending on the sample system) to compensate for such a deformation by a patterned film.

I my opinion, the most promising approach would be a compensation of the gravitational influence by turning the sample upside down after such an annealing duration that in the end a flat sample would be obtained if it were not for the film. For this, a three-point bearing and the possibility of reproducible positioning are needed. I expect that gravity would still lead to an irreversible deformation of the sample surface at the contact points. Therefore, the contact should happen at positions, e. g. close to the rim, where these deformations do not restrict the later application of the deformed part.

Besides gravity, precision is of course limited by the underlying statistical variation and measurement uncertainty in the parameter values. While the measurement 
uncertainty can, in certain cases, be avoided by direct calibration of the deformation in dependence on time, the statistical variations directly affect the precision of the process. Because viscosity depends extremely nonlinear on temperature, the temporal variation, the spatial variation and the reproducibility of the processing temperature are important parameters. To give an example: Eq. (12) in [100, p. 14] states the viscosity of water-free vitreous silica in the temperature range of 1000 to $1400{ }^{\circ} \mathrm{C}$. This equation is plotted in Fig. 5.30 for a small temperature range around the annealing point at $10^{12} \mathrm{~Pa} \cdot \mathrm{s}$. Already a change of $1 \mathrm{~K}$ in temperature leads to a change of viscosity in the range of a few percent. This deviation would be directly transferred to the sample curvature according to the viscoelastic Stoney Eq. 4.7).

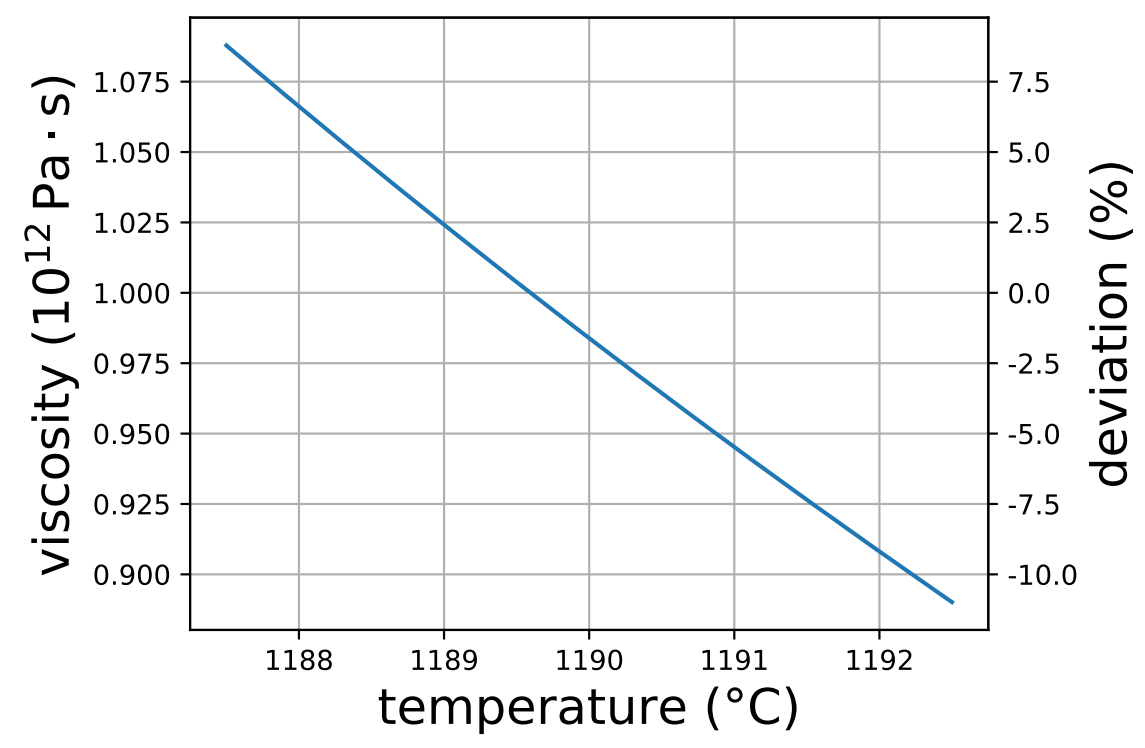

Figure 5.30: Viscosity in dependence on temperature for water-free vitreous silica according to Eq. (12) of [100, p. 14].

Considering the above discussed effects, I believe that roughly a precision of the method in the range of $1 \%$ of the target curvature could be reached with effort for a single cycle process (film patterning and subsequent annealing). To increase the precision, an iterative approach or in-situ monitoring (and also in-situ patterning) were already suggested in [90]. The iterative approach is time consuming and demands a good solution for repositioning the sample for irradiation and possibly also for annealing. The in-situ approach is challenging from a technological point of view because a special setup, combining oven, optical form measurement and possibly optical setup for laser ablation, needs to be realized. Either way, as a last processing step, figure correction by elastic deformation could be applied.

Usually, freeform optics are produced by cutting, grinding, polishing or moulding [91. One of the advantages of the deformation by a stressed film is that polishing can be performed on the flat surface before deformation. Another advantage is that it is more flexible than moulding because no mould needs to be produced first. Further, the patterning of the film can be achieved fast via laser ablation. And the annealing process, that would possibly take a considerable amount of time, can be parallelized and easily automated. However, to reach a precision of a few $10 \mathrm{~nm}$, 
as it can be achieved by grinding and polishing [91], could be difficult if surface height variations in the range of tens of micrometers are targeted. Additionally, while a process that is based on mechanical material removal can be easily applied to different glass compositions, application of the viscous deformation process on different glasses would presumably require a lot of effort. This is because of the complex behavior of film and substrate during annealing. For the film, a lot of processes can influence the stress evolution. For the case of $\mathrm{SiO}_{x}$, they are discussed in section 5.3.1. And for the substrate, the viscoelastic properties need to be known or calibrated. Unfortunately, for a precise description of the viscoelastic deformation also delayed elasticity and dilatational relaxation should be considered $[118$, Ch. 3]. Additionally, during annealing the fictive temperature and therefore the properties of the glass are changed [118, Ch. 9]. Relaxation processes in the glass are also influenced by diffusion of water inside the glass matrix $[187-189]$, so that the environment during annealing would need to be considered.

In summary, this thesis is a first step towards an application of the viscoelastic deformation by a non-continuous film for forming of glass plates. However, the realization of such a process is a difficult task. Presumably, the major issue would be the precision in the final deformation, which, to my understanding, can only be improved by considerable effort. However, further efforts could not only yield a useful process for forming of substrates but would also contribute to a better understanding of the underlying mechanisms.

\subsubsection{Outlook}

Because the viscous deformation of glass plates by a continuous or patterned film is, to the best of my knowledge, mostly unexplored, there are a lot of open questions that should be addressed. Two of those questions are related to Eqs. 4.35 and (4.45) I derived in section 4.1. Eq. 4.35) states that if the film stress is not constantly renewed during viscous deformation, a rather small curvature limit exists. It would be fascinating to study to what extent this prediction can be reproduced in the experiment. For the final curvature ratio in case of a line patterned film, I derived Eq. (4.45). Also for this equation, it would be interesting to see how well it corresponds to a well defined experiment and how the theory can be improved.

In the elastic case, for large substrate deflections, a non-uniform curvature and a bifurcation of the curvature in orthogonal directions are observed [1, 13, 15, 16]. A relevant question is if, and under which conditions, such effects can be observed in the case of viscous flow of the substrate. Further, it could be argued that the concave sample surface would develop some waviness at large curvatures due to the compressive stress inside the surface-near material. I would like to study if such an effect can be observed in the experiment.

Unfortunately, tactile form measurement, as applied in this work, is a slow process. Therefore, in possibly following projects, an optical measurement technique, like measurement of the reflected wavefront [190] or pattern projection [1], should be realized and applied. For measurement of the deformation dynamics, the ther- 
mal film stress, or the stress change during annealing, an in-situ measurement setup for the sample curvature could be used. Such a system can be simply realized by measurement of the direction of two or more laser beams reflected at the sample surface inside the oven [13, 191].

My experimental results also indicate a complex behavior of the stress in a $\mathrm{SiO}_{x}$ film during annealing. It was argued that there is a large influence of the stresses inside the material on the phase separation mechanism [161, 171, 192]. Further, oxidation could be used to control the size of the silicon nanoparticles [178]. Therefore, the idea arose that the stress distribution inside a patterned and subsequently annealed $\mathrm{SiO}_{x}$ film could be used to study these effects and even to control the technologically highly relevant optical behavior of the silicon nanoparticles on a small scale. For such experiments, laser ablation is a well suited tool because of the straight and steep film edges that can be easily produced in backside ablation and because of the possibility for blister formation by local debonding of the film [193]. The latter leads to a lateral film stress variation [194, 195] that could also be used for such experiments. 



\section{Chapter 6}

\section{Conclusion}

In excimer laser structuring of silicate glass surfaces and thin dielectric films on silicate glasses, a stress is generated by irradiation or a film stress is modified. These stresses can effect the form of the substrates and could be used for adjusting the topography of a functional surface. In the present work, I studied two different but related systems by similar experimental methods. In both cases, I applied an ArF excimer laser with a wavelength of $193 \mathrm{~nm}$ and a pulse duration of about $20 \mathrm{~ns}$ for irradiation, and measured the surface topography by tactile profilometry. For a better understanding of the experimental results, I performed simulations by the FEM.

I demonstrated the generation of long-term thermal stresses inside the borosilicate glass Schott D263M by irradiation. To the best of my knowledge, this is the second detailed study on the stress generation during excimer laser irradiation of silicate glasses and the first study that makes use of these stresses for a practical application. My results confirm and complement on the observations reported in 68 for irradiation of fused silica with an $\mathrm{F}_{2}$ laser. For stress generation, I used a mask projection setup that resulted in a flat-top beam profile at the image plane. For a large laser spot, an equibiaxial component of tensile plane stress is generated. The step-like dependence of the integrated stress on the fluence, a measurement not shown in [68], can be explained by the thermal stresses that evolve during cooling of the irradiated area. A stress of $400 \mathrm{MPa}$ is reached inside the laser affected zone, which extends up to about $650 \mathrm{~nm}$ into the material. I measured the temporal evolution of this stress, which was not considered in [68], and found that the stress decreases over time. If the surface is patterned into a line structure, an antibiaxial plane stress component is generated, which can be understood in analogy to the case of a line patterned thin film [19]. To the best of my knowledge, this is the first time that this mechanism is reported for laser structuring of a surface and irradiation induced stresses. The ability to generate equibiaxial and antibiaxial stress components on large areas could be useful in figure correction of glass substrates. For equibiaxial stress components only, I successfully made a proof-of-principle for figure correction of cover glasses of D263M. However, for long-term corrections, the stability of the generated stress needs to be improved. 
Annealing of a glass sample after deposition of a thin film can lead to a viscous deformation of the sample due to the film stress. This is the case for the system I studied, of a thin film of silicon suboxide on a plate of fused silica annealed at 1120 or $1060^{\circ} \mathrm{C}$. During annealing, the curvature of the non-coated side increases until oxidation of the film is complete. This way, much larger displacements than by an entirely elastic deformation can be reached. For patterned films, the deformation is analogous to the case of an elastic deformation, which I demonstrated by comparison to the results of a linear elastic FE simulation. This also holds for a film patterned in thin lines, for which an overall anisotropic deformation is observed. From a theoretical consideration, I predict that the curvature ratio $k_{s} / k_{p}$ of the curvatures in direction across and along the line is smaller than in the elastic case. Indeed, the derived linear viscoelastic theory is a better match to the data than the elastic equation. Unfortunately, comparison of theory and experiment is hampered by the influence of gravity and the stress distribution inside the film during annealing. Although the film stress is presumably caused by oxidation of the silicon suboxide film, an approximately linear curvature evolution with annealing time is observed. My results demonstrate that complex deformations of the fused silica plate can be generated, and the analogous behavior of the elastic and the viscous deformation facilitates the prediction of the viscous deformation for a specific film pattern, which could be useful in applying the process for forming of glass plates. However, for applying the process, the gravitational influence and precision are major issues that need to be addressed. 


\section{Appendix A}

\section{Material properties and constants}

Table A.1: Some constants and material properties used in this work.

\begin{tabular}{|c|c|c|c|c|}
\hline Name & Symbol & Value & & ource \\
\hline Gravitational acceleration & $g$ & $9.80665 \mathrm{~m} \mathrm{~s}^{-2}$ & 108 , & p. 1062] \\
\hline $\begin{array}{l}\text { Young's modulus of fused silica at } \\
25^{\circ} \mathrm{C}\end{array}$ & $E$ & $72.9 \mathrm{GPa}$ & 100 & p. 16] \\
\hline $\begin{array}{l}\text { Poisson's ratio of fused silica at } \\
25^{\circ} \mathrm{C}\end{array}$ & $\nu$ & 0.165 & $\overline{100}$ & p. 16] \\
\hline Mass density of fused silica & $\rho_{m}$ & $2.20 \mathrm{~g} \mathrm{~cm}^{-3}$ & 100 & p. 18] \\
\hline $\begin{array}{l}\text { Bulk modulus of fused silica at } \\
1300 \mathrm{~K}\end{array}$ & $K$ & $\approx 42.5 \mathrm{GPa}$ & 159 & Fig. 11] \\
\hline $\begin{array}{l}\text { Sheer modulus of fused silica at } \\
1300 \text { and at } 1400 \mathrm{~K}\end{array}$ & $G$ & $\approx 33 \mathrm{GPa}$ & $\overline{159}$, & Fig. 11] \\
\hline $\begin{array}{l}\text { Bulk modulus of fused silica at } \\
1400 \mathrm{~K}\end{array}$ & $K$ & $\approx 43 \mathrm{GPa}$ & $\overline{159}$, & Fig. 11] \\
\hline $\begin{array}{l}\text { Linear thermal expansion coeffi- } \\
\text { cient of vitreous silica }\end{array}$ & $\alpha$ & $\approx 0.5 \cdot 10^{-6} \mathrm{~K}^{-1}$ & 100 & Tab. 2.13] \\
\hline $\begin{array}{l}\text { Glass transition temperature of } \\
\text { vitreous silica }\end{array}$ & $T_{g}$ & $\approx 1150^{\circ} \mathrm{C}$ & 95 & p. 16] \\
\hline $\begin{array}{l}\text { Young's modulus of } \text { Schott } \\
\text { D263M }\end{array}$ & $E$ & $72.9 \mathrm{GPa}$ & & 128 \\
\hline Poisson's ratio of Schott D263M & $\nu$ & 0.208 & & 128 \\
\hline $\begin{array}{l}\text { Linear thermal expansion coef- } \\
\text { ficient of Schott D263M ( } 20- \\
\left.300^{\circ} \mathrm{C}\right)\end{array}$ & $\alpha$ & $7.2 \cdot 10^{-6} \mathrm{~K}^{-1}$ & & 128 \\
\hline $\begin{array}{l}\text { Transformation temperature of } \\
\text { Schott D263M }\end{array}$ & $T_{g}$ & $557^{\circ} \mathrm{C}$ & & 128 \\
\hline Mass density of Schott D263M & $\rho_{m}$ & $2.51 \mathrm{~g} / \mathrm{cm}^{3}$ & & 128 \\
\hline $\begin{array}{l}\text { Thermal conductivity of Schott } \\
\text { D } 263 \mathrm{~T} \text { eco at } 90^{\circ} \mathrm{C}\end{array}$ & $\lambda_{c}$ & $1.06 \mathrm{~W} /(\mathrm{m} \cdot \mathrm{K})$ & & 196 \\
\hline $\begin{array}{l}\text { Mean specific heat capacity of } \\
\text { Schott D263 bio at } 20 \text { to } 100^{\circ} \mathrm{C}\end{array}$ & $c_{p}$ & $0.8 \mathrm{~J} /(\mathrm{g} \cdot \mathrm{K})$ & & 197 \\
\hline Poisson's ratio of $\mathrm{SiO}$ film & $\nu$ & 0.17 & & 164 \\
\hline
\end{tabular}





\section{Appendix B}

\section{Evaluation of $\mathcal{L}[I(v)]$ in Eq. (4.16)}

In section 4.1.1, the value of the integral

$$
\begin{aligned}
\mathcal{L}[I(v)] & =\int_{0}^{\infty} \exp (-p v) I(v) \mathrm{d} v \\
& =\int_{0}^{\infty}\left(\exp \left[-\left(p+\tau_{s} / \tau_{f}\right) v\right] \int_{0}^{v \cdot \tau_{s}} \exp \left(s / \tau_{f}\right) \frac{1}{\sqrt{s}} \mathrm{~d} s\right) \mathrm{d} v \\
& =\frac{\sqrt{\pi \cdot \tau_{s}}}{\sqrt{p}\left(p+\tau_{s} / \tau_{f}\right)}
\end{aligned}
$$

is used in Eq. (4.17). In the present section, I demonstrate the above equality.

By partial integration of Eq. B.2

$$
\begin{aligned}
\mathcal{L}[I(v)] & =\underbrace{-\left.\frac{1}{p+\tau_{s} / \tau_{f}} \exp \left[-\left(p+\frac{\tau_{s}}{\tau_{f}}\right) v\right] \int_{0}^{v \cdot \tau_{s}} \frac{\exp \left(s / \tau_{f}\right)}{\sqrt{s}} \mathrm{~d} s\right|_{v=0} ^{v=\infty}}_{=S_{1}} \\
& +\underbrace{\frac{\sqrt{\tau_{s}}}{p+\tau_{s} / \tau_{f}} \int_{0}^{\infty} \exp \left[-\left(p+\frac{\tau_{s}}{\tau_{f}}\right) v\right] \frac{\exp \left[v \cdot \tau_{s} / \tau_{f}\right]}{\sqrt{v}} \mathrm{~d} v}_{=S_{2}}
\end{aligned}
$$

is obtained.

First, I show that $S_{1}=0$ : The upper limit of $S_{1}$ is

$$
\begin{aligned}
S_{1, \infty} & =\lim _{v \rightarrow \infty}-\frac{1}{p+\tau_{s} / \tau_{f}} \exp \left[-\left(p+\frac{\tau_{s}}{\tau_{f}}\right) v\right] \int_{0}^{v \cdot \tau_{s}} \frac{\exp \left(s / \tau_{f}\right)}{\sqrt{s}} \mathrm{~d} s \\
& \propto \lim _{v \rightarrow \infty} \frac{\int_{0}^{v \cdot \tau_{s}} \frac{\exp \left(s / \tau_{f}\right)}{\sqrt{s}} \mathrm{~d} s}{\exp \left[\left(p+\frac{\tau_{s}}{\tau_{f}}\right) v\right]} \\
& \stackrel{\text { L'Hospital }}{=} \lim _{v \rightarrow \infty} \frac{\sqrt{\tau_{s}}}{p+\tau_{s} / \tau_{f}} \frac{\exp (-p v)}{\sqrt{v}} \\
& =0
\end{aligned}
$$


where L'Hospital's rule was used in the third row. The lower limit of $S_{1}$ is

$$
\begin{aligned}
S_{1,0} & =\lim _{v \rightarrow+0}-\frac{1}{p+\tau_{s} / \tau_{f}} \exp \left[-\left(p+\frac{\tau_{s}}{\tau_{f}}\right) v\right] \int_{0}^{v \cdot \tau_{s}} \frac{\exp \left(s / \tau_{f}\right)}{\sqrt{s}} \mathrm{~d} s \\
& \propto \lim _{v \rightarrow+0} \int_{0}^{v \cdot \tau_{s}} \frac{\exp \left(s / \tau_{f}\right)}{\sqrt{s}} \mathrm{~d} s \\
& =\lim _{v \rightarrow+0}\left[\left.2 \sqrt{s} \exp \left(s / \tau_{f}\right)\right|_{0} ^{v \cdot \tau_{s}}-\frac{2}{\tau_{f}} \int_{0}^{v \cdot \tau_{s}} \sqrt{s} \exp \left(s / \tau_{f}\right) \mathrm{d} s\right] \\
& =0,
\end{aligned}
$$

where partial integration was used in the third row. Thus, $S_{1}=S_{1, \infty}-S_{1,0}=0$.

Second, $S_{2}$ is calculated by noticing that

$$
\begin{aligned}
S_{2} & =\frac{\sqrt{\tau_{s}}}{p+\tau_{s} / \tau_{f}} \int_{0}^{\infty} \frac{\exp (-p v)}{\sqrt{v}} \mathrm{~d} v \\
& =\frac{\sqrt{\tau_{s}}}{p+\tau_{s} / \tau_{f}} \mathcal{L}\left[\frac{1}{\sqrt{v}}\right] \\
& =\sqrt{\pi \cdot \tau_{s}} \frac{1}{\sqrt{p}} \frac{1}{p+\tau_{s} / \tau_{f}} .
\end{aligned}
$$

In the last step, I used a conversion table [108, p. 1120] for the Laplace transformation.

All together, it follows that

$$
\mathcal{L}[I(v)]=S_{2}=\sqrt{\pi \cdot \tau_{s}} \frac{1}{\sqrt{p}} \frac{1}{p+\tau_{s} / \tau_{f}} .
$$




\section{Appendix $\mathrm{C}$}

\section{Annealing in air at $1120^{\circ} \mathrm{C}$ with a macroscopic line pattern}

\section{C.1 Horizontal sample orientation}

In section 5.1.1, I presented the results for the viscous deformation of a fused silica plate in horizontal orientation. The deformation is caused by a $\mathrm{SiO}_{x}$ film that has been removed on one half of the coated surface via laser ablation. In the current section, I show similar results for a different film structure.

The sample consisted of a cylindrical Heraeus Suprasil fused silica plate of $1078 \mu \mathrm{m}$ thickness and a diameter of $25 \mathrm{~mm}$, of which one side was covered by a $\mathrm{SiO}_{x}$ film with a thickness of 650(10) nm after deposition. Via backside ablation with the light of an ArF excimer laser, I structured the film into a stripe pattern with a period of about $4 \mathrm{~mm}$ and a duty cycle of 0.5 (Fig. C.1f). The roughly quadratic laser spot was formed by a homogenizer setup (cf. Fig. 2.2) and exhibited an edge length of about $1 \mathrm{~mm}$. To achieve the stripe pattern, the spot was stitched with overlap and one pulse per position was applied. The fluence was about $500 \mathrm{~mJ} / \mathrm{cm}^{2}$. After patterning, the sample was first annealed in nitrogen flow at $900{ }^{\circ} \mathrm{C}$ and subsequently for different durations in air at $1120(1)^{\circ} \mathrm{C}$ in horizontal orientation (cf. Fig. 2.9a).

The height profiles of the non-coated sample surface are plotted in Figs. C.1a and C.1b for direction across and along the lines, respectively, for different total annealing times. The overall deflection is roughly the same in both directions. However, in direction across the lines, at the position of the residual film stripes, a local concave deformation is observed, while in direction along the lines, the height profiles exhibit an approximately parabolic shape.

The difference between both directions becomes more pronounced in the curvature profiles in Figs. C.1c and C.1d for directions across and along the lines, respectively. The curvature profiles after the preliminary annealing step at $900{ }^{\circ} \mathrm{C}$ have been subtracted. Across the lines, at the position of the residual stripes, the curvature becomes positive, while in the decoated regions it becomes negative. Ad- 


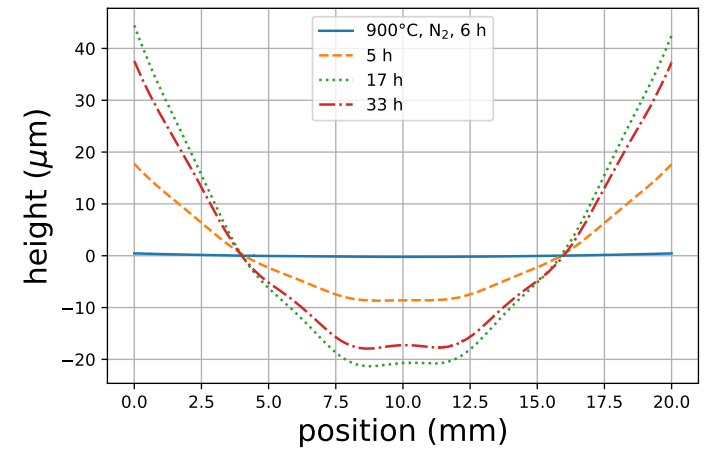

(a) across the lines

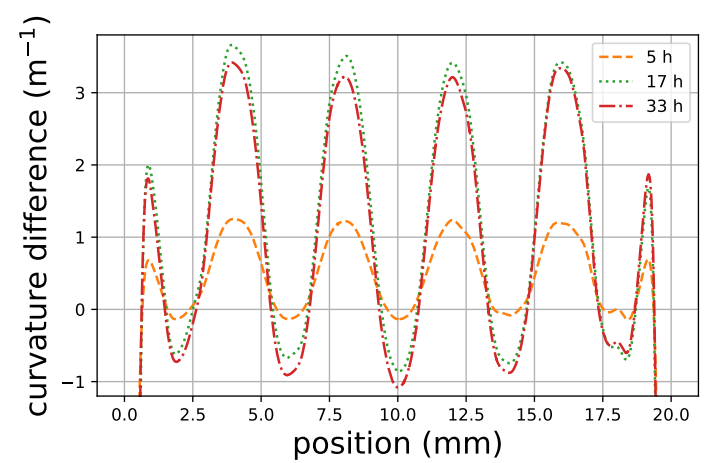

(c) across the lines

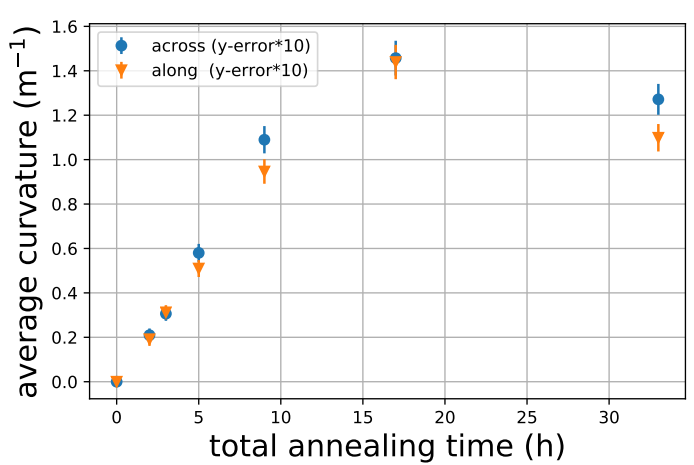

(e)

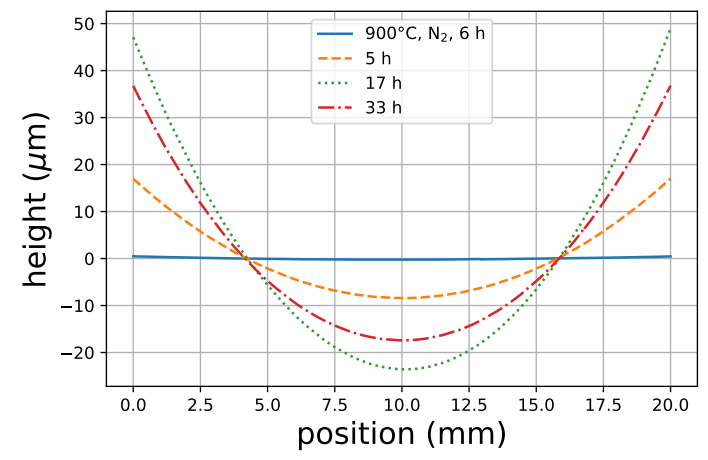

(b) along the lines

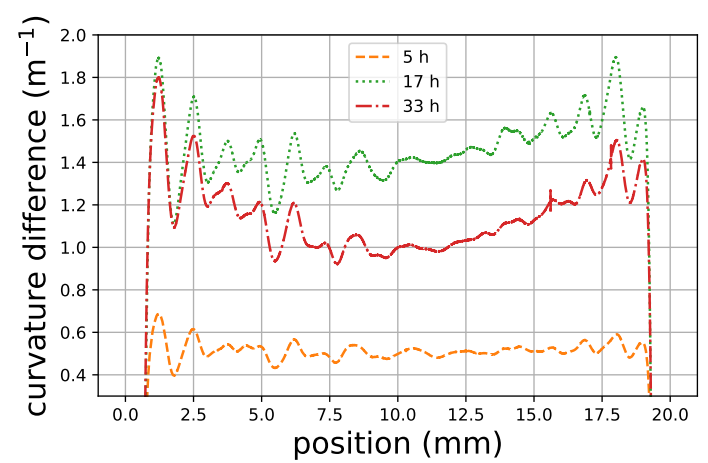

(d) along the lines

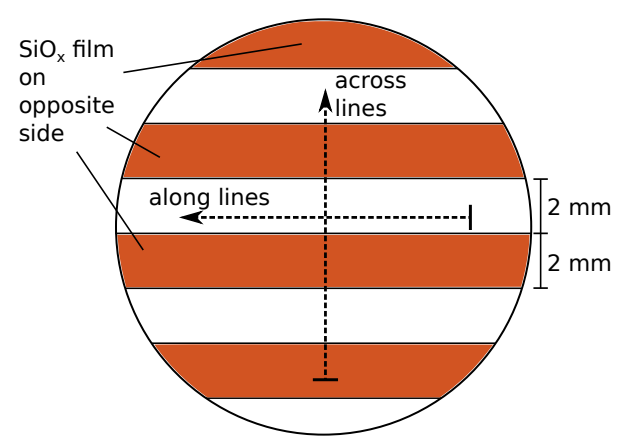

(f)

Figure C.1: Experimental results for a fused silica plate covered by a stripe pattern of $650(10) \mathrm{nm}$ thick $\mathrm{SiO}_{x}$ and annealing at $1120(1)^{\circ} \mathrm{C}$ in air in horizontal sample orientation. (a,b) The height profiles in directions across and along the stripes. (c,d) The corresponding curvature profiles. (e) The average curvature values calculated from the curvature profiles. (f) A schematic of the sample with the non-coated side facing the reader. 
ditionally, with increasing annealing time an overall concave shape of the curvature profiles develops.

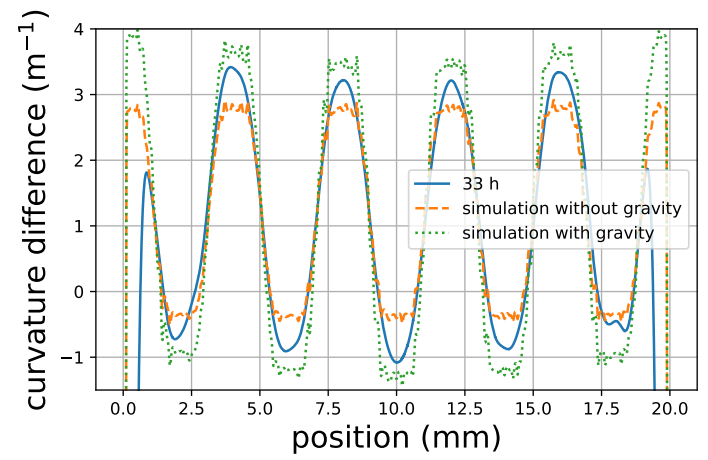

(a) across the lines

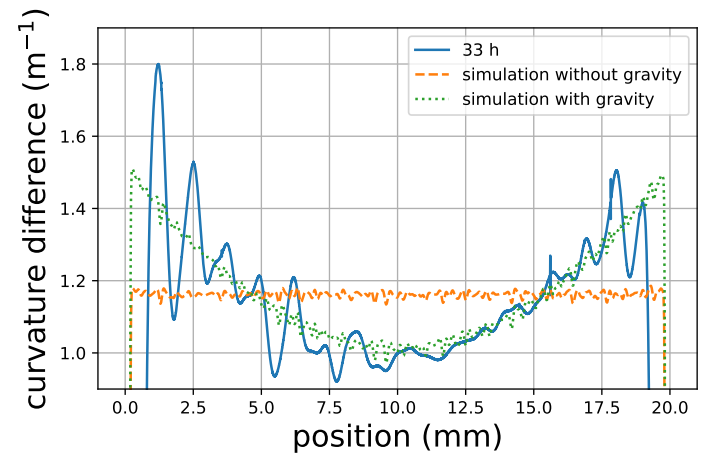

(b) along the lines

Figure C.2: Comparison of the curvature profiles after $33 \mathrm{~h}$ of annealing for the sample of Fig. C.1 with the results of a linear elastic FE simulation excluding and including gravity.

Fig. C.1e shows the average values of the curvature profiles in between 2.5 and $17.5 \mathrm{~mm}$ in dependence on the total annealing time. Up to $9 \mathrm{~h}$ of total annealing time, the average curvature increases approximately linearly. After $17 \mathrm{~h}$, after oxidation is complete, it decreases again.

For reproduction of the experimental results after $33 \mathrm{~h}$ of annealing, I performed a linear elastic FE simulation with and without the influence of gravity. A substrate thickness of $t_{s}=1078 \mu \mathrm{m}$ and a film thickness of $t_{f}=651 \mathrm{~nm}$ were used in the simulation. For the simulation excluding gravitational effects, the hypothetical film stress was estimated by Eq. (3.1) via the experimentally determined average curvature to a value of $-61.54 \mathrm{GPa}$. For the simulation including gravity, I manually varied film stress and mass density for apparently best fit to the experimental data. This resulted in a hypothetical film stress of $-92 \mathrm{GPa}$ and a hypothetical density of $20 \mathrm{~kg} / \mathrm{cm}^{3}$.

A comparison of the curvature profiles for $33 \mathrm{~h}$ of annealing with the simulation results is shown in Fig. C.2. The simulation excluding gravity cannot describe the underlying concave shape of the curvature profiles in both directions. Plus, it underestimates the amplitude of the curvature profiles in direction across the lines (Fig. C.2a). In contrast, concave curvature profiles and amplitude are well described by the simulation including gravity.

The results presented in this section are in good agreement with the ones of section 5.1.1 for a half decoated sample. Therefore, they confirm the observations described therein. 


\section{C.2 Vertical orientation}

I also annealed a sample with a similar film pattern as in the preceding section (Fig. C.1f) in vertical orientation (cf. Fig. 2.9b). This time, the thickness of the film was 499(4) nm and the thickness of the substrate $1.083 \mathrm{~mm}$. The $\mathrm{SiO}_{x}$ film was structured in backside ablation with a $200 \cdot 200 \mu \mathrm{m}^{2}$ laser spot, which was achieved via mask projection (cf. Fig. 2.1) at a fluence of about $500 \mathrm{~mJ} / \mathrm{cm}^{2}$ and one pulse per position. The spot was scanned with an overlap of $10 \mu \mathrm{m}$.

The height profiles after selected total annealing times are plotted in Figs. C.3a and C.3b across and along the lines, respectively. They are comparable to the ones in Fig. C.1 for horizontal annealing. A difference can be observed in the curvature profiles in direction across the lines in Fig. C.3c. Here, no evolution of an underlying concave shape can be observed. The largest differences to annealing in horizontal orientation are observed in Fig. C.3e for the time dependence of the average curvatures. They split after $9 \mathrm{~h}$ of annealing and thereupon the curvature across the lines increases while the curvature along the lines decreases. A similar behavior was already observed in Fig. 5.5e for vertical annealing of a sample with a different film pattern.

Fig. C.4 shows a comparison of the experimental results after $33 \mathrm{~h}$ of annealing with the results of a linear elastic FE simulation excluding gravity. From the average curvatures, a hypothetical film stress of -116.796 GPa was calculated and assumed in the simulation. The concave shape of the experimental curvature profile in direction along the lines cannot be reproduced by the simulation. And in direction across the lines, the maxima of the curvature profiles are underestimated.

The obtained results are in agreement with the ones I presented in section 5.1 .2 for a sample with a half decoated $\mathrm{SiO}_{x}$ film, that was treated analogously. Therefore, they confirm these results. 


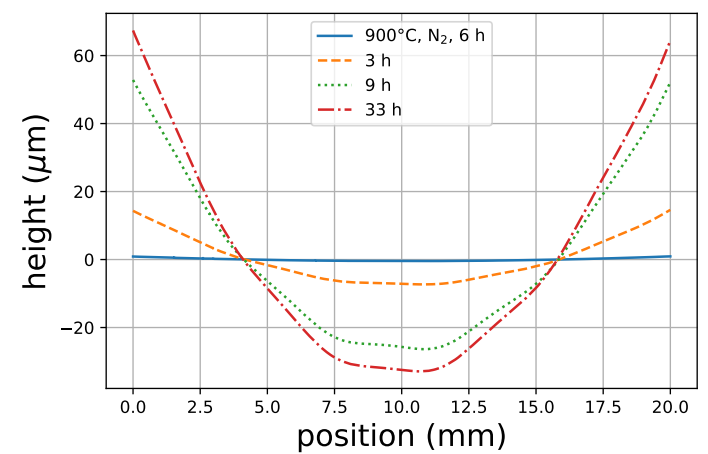

(a) across the lines

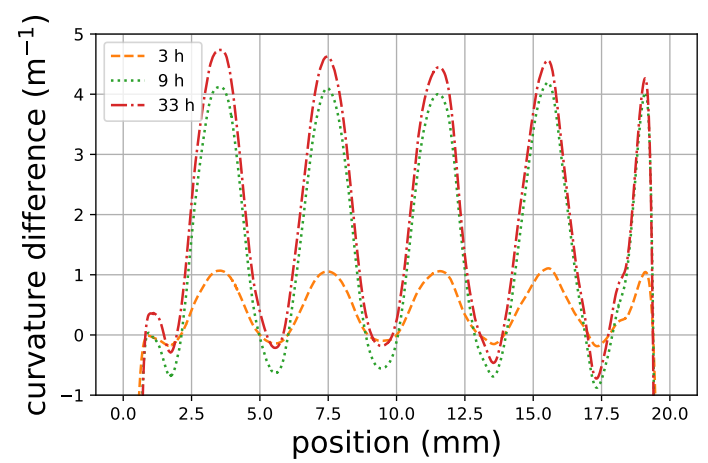

(c) across the lines

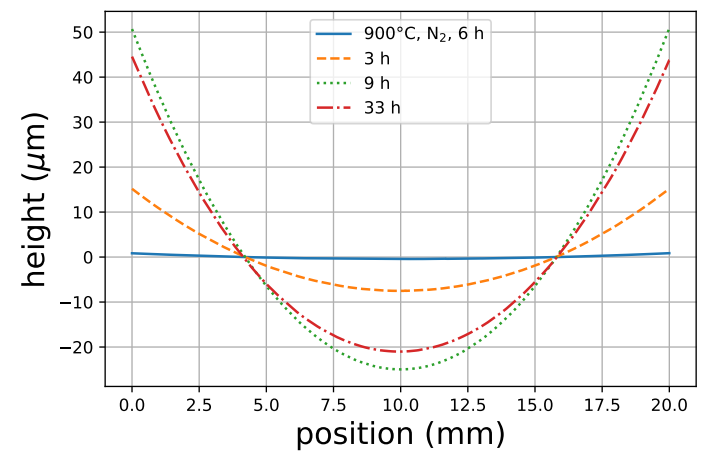

(b) along the lines

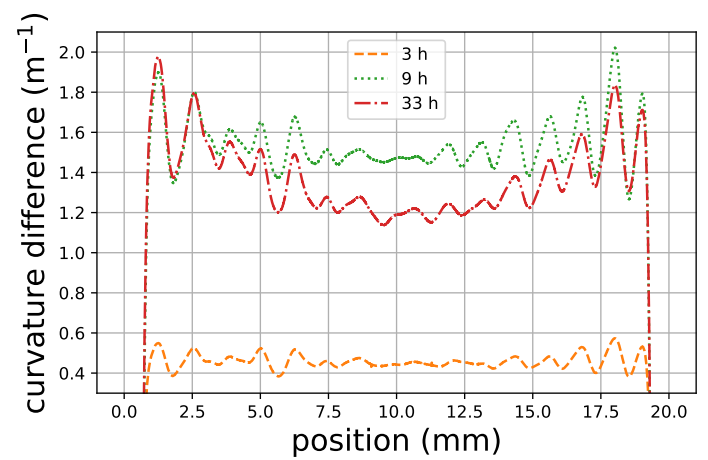

(d) along the lines

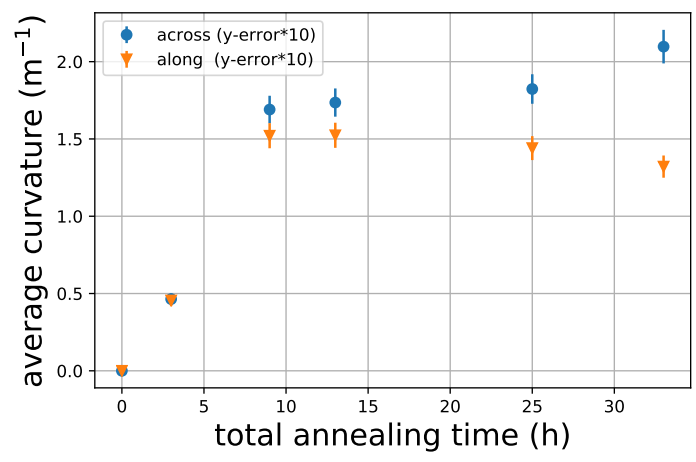

(e)

Figure C.3: Experimental results for a fused silica plate covered by a stripe pattern of 499(4) nm thick $\mathrm{SiO}_{x}$ and annealing at $1120(1){ }^{\circ} \mathrm{C}$ in air in vertical orientation. $(\mathrm{a}, \mathrm{b})$ The height profiles in directions across and along the stripes. (c,d) The corresponding curvature profiles. (e) The average curvature values calculated from the curvature profiles. 


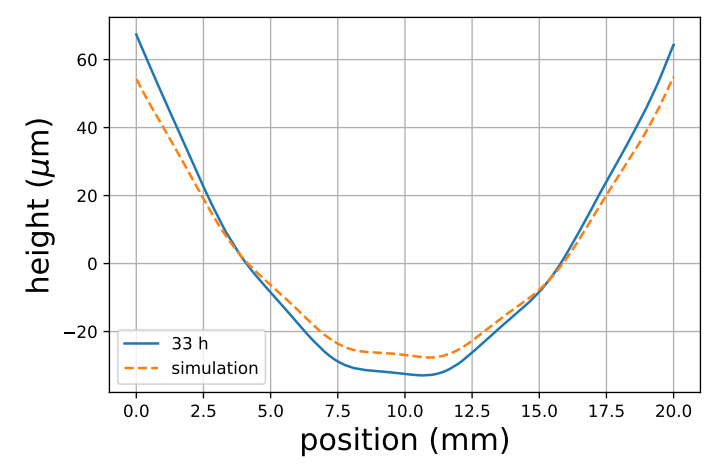

(a) across the lines

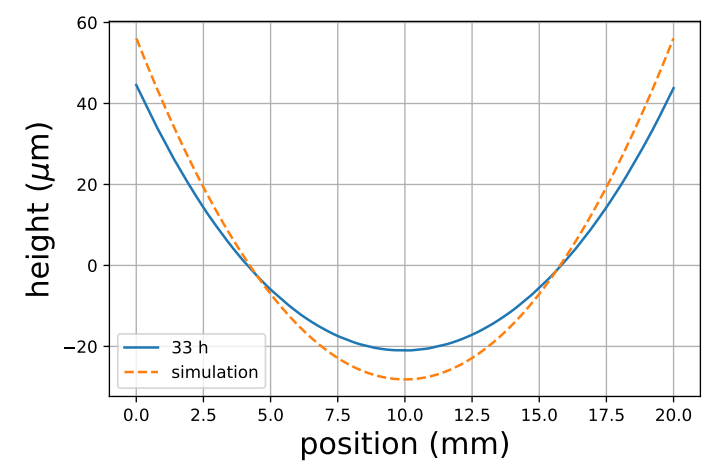

(b) along the lines

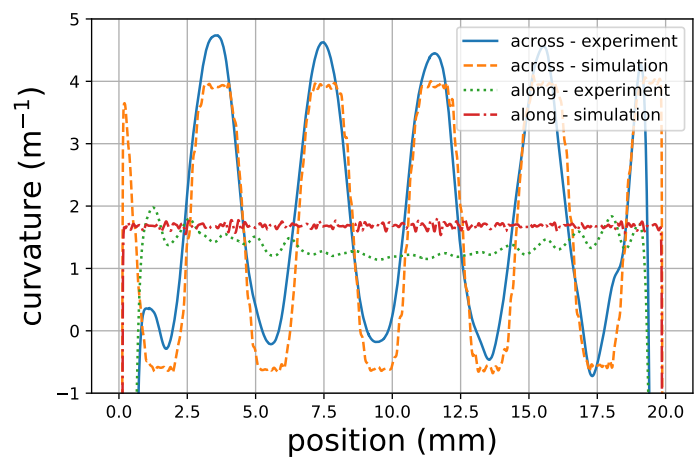

(c)

Figure C.4: Comparison of the height profiles (a,b) and the curvature profiles (c) after $33 \mathrm{~h}$ of annealing for the sample of Fig. C.3 with the results of a linear elastic FE simulation. 


\section{Bibliography}

[1] M. Finot, I. A. Blech, S. Suresh, and H. Fujimoto. "Large deformation and geometric instability of substrates with thin-film deposits". In: Journal of Applied Physics 81.8 (1997), pp. 3457-3464.

[2] B. E. Deal and A. S. Grove. "General Relationship for the Thermal Oxidation of Silicon". In: Journal of Applied Physics 36.12 (1965), pp. 3770-3778.

[3] A. Wikström, P. Gudmundson, and S. Suresh. "Thermoelastic analysis of periodic thin lines deposited on a substrate". In: Journal of the Mechanics and Physics of Solids 47.5 (1999), pp. 1113-1130.

[4] Georg N. Strauss. "Mechanical Stress in Optical Coatings". In: Optical Interference Coatings. Ed. by Norbert Kaiser and Hans K. Pulker. Springer, 2003, pp. 207-229.

[5] Pete Kupinski. "Laser mirror design tradeoffs impact optical system performance". In: Laser Focus World (Nov. 2019), pp. 29-34.

[6] J. Priest, H. L. Caswell, and Y. Budo. "Mechanical stresses in silicon oxide films". In: Vacuum 12.6 (1962), pp. 301-306.

[7] Gustavo Gioia and Michael Ortiz. "Delamination of Compressed Thin Films". In: Advances in Applied Mechanics 33 (1997), pp. 119-192.

[8] A. A. Volinsky and P. Waters. "Delaminated Film Buckling Microchannels". In: Mechanical Self-Assembly: Science and Applications. Ed. by X. Chen. Springer, 2013.

[9] Stephen Gensemer and Mark Gross. "Figuring large optics at the sub-nanometer level: compensation for coating and gravity distortions". In: Opt. Express 23.24 (Nov. 2015), pp. 31171-31180.

[10] Brandon D. Chalifoux, Youwei Yao, Ralf K. Heilmann, and Mark L. Schattenburg. "Simulations of film stress effects on mirror segments for the Lynx X-ray Observatory concept". In: Journal of Astronomical Telescopes, Instruments, and Systems 5.2 (2019), p. 021004.

[11] Gerald Stoney. "The Tension of Metallic Films deposited by Electrolysis". In: Proceedings of the Royal Society A: Mathematical, Physical and Engineering Sciences 82 (553 1909), pp. 172-175.

[12] Abner Brenner and Seymour Senderoff. "Calculation of Stress in Electrodeposits from the Curvature of a Plated Strip". In: Journal of Research of the National Bureau of Standards 42 (1949), pp. 105-123. 
[13] D. E. Fahnline, Christine B. Masters, and N. J. Salamon. "Thin film stress from nonspherical substrate bending measurements". In: Journal of Vacuum Science \& Technology A 9.4 (1991), pp. 2483-2487.

[14] P. H. Townsend, D. M. Barnett, and T. A. Brunner. "Elastic relationships in layered composite media with approximation for the case of thin films on a thick substrate". In: Journal of Applied Physics 62.11 (1987), pp. 4438-4444.

[15] Brian D. Harper and Wu Chih-Ping. "A geometrically nonlinear model for predicting the intrinsic film stress by the bending-plate method". In: International Journal of Solids and Structures 26.5 (1990), pp. 511 -525.

[16] Christine B. Masters and N.J. Salamon. "Geometrically nonlinear stressdeflection relations for thin film/substrate systems". In: International Journal of Engineering Science 31.6 (1993), pp. 915-925.

[17] D. Ngo, X. Feng, Y. Huang, A.J. Rosakis, and M.A. Brown. "Thin film/substrate systems featuring arbitrary film thickness and misfit strain distributions. Part I: Analysis for obtaining film stress from non-local curvature information". In: International Journal of Solids and Structures 44.6 (2007). Physics and Mechanics of Advanced Materials, pp. 1745-1754.

[18] M.A. Brown, A.J. Rosakis, X. Feng, Y. Huang, and Ersan Üstündag. "Thin film/substrate systems featuring arbitrary film thickness and misfit strain distributions. Part II: Experimental validation of the non-local stress/curvature relations". In: International Journal of Solids and Structures 44.6 (2007). Physics and Mechanics of Advanced Materials, pp. 1755-1767.

[19] Y.-L. Shen, S. Suresh, and I. A. Blech. "Stresses, curvatures, and shape changes arising from patterned lines on silicon wafers". In: Journal of Applied Physics 80.3 (1996), pp. 1388-1398.

[20] A. Gouldstone, Y.-L. Shen, S. Suresh, and C. V. Thompson. "Evolution of stresses in passivated and unpassivated metal interconnects". In: Journal of Materials Research 13.7 (1998), pp. 1956-1966.

[21] Peter Gudmundson and Zang Weilin. "An analytic model for thermoelastic properties of composite laminates containing transverse matrix cracks". In: International Journal of Solids and Structures 30.23 (1993), pp. 3211-3231.

[22] Kai-Wing Chan, Marton Sharpe, William Zhang, Linette Kolos, Melinda Hong, Ryan McClelland, Bruce Hohl, Timo Saha, and James Mazzarella. "Coating thin mirror segments for lightweight x-ray optics". In: Proceedings of SPIE 8861 (2013), p. 88610X.

[23] Martin Bischoff, Tobias Nowitzki, Oliver Voß, Steffen Wilbrandt, and Olaf Stenzel. "Postdeposition treatment of IBS coatings for UV applications with optimized thin-film stress properties". In: Appl. Opt. 53.4 (Feb. 2014), A212A220.

[24] Hideyuki Mori, Takashi Okajima, William W. Zhang, Kai-Wing Chan, Richard Koenecke, James R. Mazzarella, Ai Numata, Lawrence G. Olsen, Raul E. Riveros, and Mihoko Yukita. "Reflective coatings for the future x-ray mirror substrates". In: Proceedings of SPIE 10699 (2018), p. 1069941.

[25] Webpage of the LYNX x-ray observatory. https://www. lynxobservatory. com. Accessed June 1, 2021. 
[26] John C. Lambropoulos, Su Xu, Tong Fang, and Donald Golini. "Twyman effect mechanics in grinding and microgrinding". In: Appl. Opt. 35.28 (Oct. 1996), pp. 5704-5713.

[27] Stephen L. O’Dell, Ryan Allured, Andrew O. Ames, Michael P. Biskach, David M. Broadway, Ricardo J. Bruni, David N. Burrows, Jian Cao, Brandon D. Chalifoux, Kai-Wing Chan, Yip-Wah Chung, Vincenzo Cotroneo, Ronald F. Elsner, Jessica A. Gaskin, Mikhail V. Gubarev, Ralf K. Heilmann, Edward Hertz, Thomas N. Jackson, Kiranmayee Kilaru, Jeffrey J. Kolodziejczak, Ryan S. McClelland, Brian D. Ramsey, Paul B. Reid, Raul E. Riveros, Jacqueline M. Roche, Suzanne E. Romaine, Timo T. Saha, Mark L. Schattenburg, Daniel A. Schwartz, Eric D. Schwartz, Peter M. Solly, Susan Trolier-McKinstry, Melville P. Ulmer, Alexey Vikhlinin, Margeaux L. Wallace, Xiaoli Wang, David L. Windt, Youwei Yao, Shi Ye, William W. Zhang, and Heng Zuo. "Toward large-area sub-arcsecond x-ray telescopes II". In: Proceedings of SPIE 9965 (2016), p. 996507.

[28] Mireille Akilian, Craig R. Forest, Alexander H. Slocum, David L. Trumper, and Mark L. Schattenburg. "Thin optic constraint". In: Precision Engineering 31.2 (2007), pp. 130-138.

[29] Stemmed mirrors in the online catalog of Edmund Optics. https : / / www . edmundoptics.eu/f/stemmed-mirrors/39665/. Accessed June 1, 2021.

[30] Michael Zeuner and Sven Kiontke. "Ion Beam Figuring Technology in Optics Manufacturing". In: Optik \& Photonik 7.2 (2012), pp. 56-58.

[31] J. B. Oliver, J. Spaulding, and B. Charles. "Stress compensation by deposition of a nonuniform corrective coating". In: Appl. Opt. 59.5 (Feb. 2020), A54-A57.

[32] Yingna Shi, Qiushi Huang, Runze Qi, Zhengxiang Shen, Zhong Zhang, and Zhanshan Wang. "Theoretical and Experimental Study of Particle Distribution from Magnetron Sputtering with Masks for Accurate Thickness Profile Control". In: Coatings 10.4 (2020).

[33] Youwei Yao, Xiaoli Wang, Jian Cao, and Melville Ulmer. "Stress manipulated coating for fabricating lightweight X-ray telescope mirrors". In: Opt. Express 23.22 (Nov. 2015), pp. 28605-28618.

[34] Casey T. DeRoo, Ryan Allured, Vincenzo Cotroneo, Edward N. Hertz, Vanessa Marquez, Paul B. Reid, Eric D. Schwartz, Alexey A. Vikhlinin, Susan TrolierMcKinstry, Julian Walker, Thomas N. Jackson, Tianning Liu, and Mohit Tendulkar. "Deterministic figure correction of piezoelectrically adjustable slumped glass optics". In: Journal of Astronomical Telescopes, Instruments, and Systems 4.1 (2018), p. 019004.

[35] Heng E. Zuo, Brandon D. Chalifoux, Ralf K. Heilmann, and Mark L. Schattenburg. "Ultrafast laser micro-stressing for correction of thin fused silica optics for the Lynx X-Ray Telescope Mission". In: Proceedings of SPIE 10699 (2018), p. 1069954.

[36] Brandon D. Chalifoux, Youwei Yao, Kevin B. Woller, Ralf K. Heilmann, and Mark L. Schattenburg. "Compensating film stress in thin silicon substrates using ion implantation". In: Opt. Express 27.8 (Apr. 2019), pp. 11182-11195. 
[37] Youwei Yao, Brandon D. Chalifoux, Ralf K. Heilmann, and Mark L. Schattenburg. "Thermal oxide patterning method for compensating coating stress in silicon substrates". In: Opt. Express 27.2 (Jan. 2019), pp. 1010-1024.

[38] Brandon D. Chalifoux, Ralf K. Heilmann, and Mark L. Schattenburg. "Correcting flat mirrors with surface stress: analytical stress fields". In: J. Opt. Soc. Am. A 35.10 (Oct. 2018), pp. 1705-1716.

[39] Brandon Chalifoux, Heng Zuo, Graham Wright, Youwei Yao, Ralf K. Heilmann, and Mark L. Schattenburg. "Gas bearing slumping and figure correction of x-ray telescope mirror substrates". In: Proceedings of SPIE 9905 (2016), p. 990522.

[40] J. N. Reddy. An Introduction to Continuum Mechanics. 2nd edition. Cambridge University Press, 2013.

[41] Youwei Yao, Brandon D. Chalifoux, Ralf K. Heilmann, Kai-Wing Chan, Hideyuki Mori, Takashi Okajima, William W. Zhang, and Mark L. Schattenburg. "Progress of coating stress compensation of silicon mirrors for Lynx x-ray telescope mission concept using thermal oxide patterning method". In: Journal of Astronomical Telescopes, Instruments, and Systems 5.2 (2019), pp. 1-9.

[42] Vladimir Dmitriev and Uri Stern. method and apparatus for locally deforming an optical element for photolithography. US Patent 9,606,444 B2. Mar. 2017.

[43] Yves Bellouard, Audrey Champion, Benjamin McMillen, Sebabrata Mukherjee, Robert R. Thomson, Charles Pépin, Philippe Gillet, and Ya Cheng. "Stress-state manipulation in fused silica via femtosecond laser irradiation". In: Optica 3.12 (Dec. 2016), pp. 1285-1293.

[44] M. Geiger, A. Huber, and G. Esser. Verfahren zur Positionsjustage lageempfindlicher Bauteile, insbesondere von Mikro-Bauteilen der Elektronik, Optoelektronik oder Mechantronik. German Patent, DE 10044212 B4. 2005.

[45] P. Bechthold and M. Schmidt. "Non-thermal Micro Adjustment Using Ultrashort Laser Pulses". In: Journal of Laser Micro/Nanoengineering 2.3 (2007), pp. $183-188$.

[46] Heng E. Zuo, Brandon D. Chalifoux, Ralf K. Heilmann, Sang-Hoon Nam, Kyung-Han Hong, and Mark L. Schattenburg. "Demonstration of femtosecond laser micromachining for figure correction of thin silicon optics for x-ray telescopes". In: Proceedings of SPIE 11119 (2019), 111191A.

[47] R. Dohlus. Lasertechnik. De Gruyter, 2015.

[48] M. Geiger and F. Vollertsen. "The Mechanisms of Laser Forming". In: CIRP Annals 42.1 (1993), pp. 301-304.

[49] Manfred Geiger and Frank Meyer-Pittroff. "Laser beam bending of metallic foils". In: Proceedings of SPIE 4426 (2002), pp. 187-190.

[50] X. Richard Zhang and Xianfan Xu. "Microscale bending of brittle materials using pulsed and CW lasers". In: Proceedings of SPIE 4637 (2002).

[51] Dongjiang Wu, Guangyi Ma, and Dongming Guo. "Temperature Gradient Mechanism on Laser Bending of Borosilicate Glass Sheet". In: Journal of Manufacturing Science and Engineering 132 (2010). 
[52] J. Griffiths, S.P. Edwardson, G. Dearden, and K.G. Watkins. "Thermal laser micro-adjustment using picosecond pulse durations". In: Applied Surface Science 258.19 (2012), pp. 7639-7643.

[53] Nathan Lazarus and Gabriel L. Smith. "Laser Forming for Complex 3D Folding". In: Advanced Materials Technologies 2.10 (2017), p. 1700109.

[54] Mehdi Safari and Mahmoud Farzin. "Experimental investigation of laser forming of a saddle shape with spiral irradiating scheme". In: Optics \& Laser Technology 66 (2015), pp. 146-150.

[55] Klaus Körber. "Introduction to Laser Physics and Excimer Laser Sources". In: Excimer Laser Technology: laser sources, optics, systems and applications. Ed. by Dirk Basting. Lambda Physik AG, 2001.

[56] Coherent Inc. Excimer Lasers \& UV Optical Systems - Product Catalog. 2020.

[57] R. Karstens, A. Gödecke, A. Prießner, and J. Ihlemann. "Fabrication of 250nm-hole arrays in glass and fused silica by UV laser ablation". In: Optics 86 Laser Technology 83 (2016), pp. 16-20.

[58] T. Fricke-Begemann and J. Ihlemann. "Hybrid micro-optical elements by laser-based fabrication of Fresnel lenses on the end face of gradient index lenses". In: Opt. Express 26.18 (Sept. 2018), pp. 23751-23759.

[59] Gerhard Heise, Andreas Börner, Marcel Dickmann, Marina Englmaier, Andreas Heiss, Matthias Kemnitzer, Jan Konrad, Regina Moser, Jörg Palm, Helmut Vogt, and Heinz P. Huber. "Demonstration of the monolithic interconnection on CIS solar cells by picosecond laser structuring on 30 by $30 \mathrm{~cm}^{2}$ modules". In: Progress in Photovoltaics: Research and Applications 23.10 (2015), pp. 1291-1304.

[60] Mihai Stafe, Aurelian Marcu, and Niculae N. Puscas. Pulsed Laser Ablation of Solids - Basics, Theory and Applications. Springer, 2014.

[61] C. Buerhop, R. Weissmann, and N. Lutz. "Ablation of silicate glasses by laser irradiation: modelling and experimental results". In: Applied Surface Science 54 (1992), pp. 187-192.

[62] J. Ihlemann, B. Wolff, and P. Simon. "Nanosecond and Femtosecond Excimer Laser Ablation of Fused Silica". In: Applied Physics A 54 (1992), pp. 363368.

[63] P.R. Herman, R.S. Marjoribanks, A. Oettl, K. Chen, I. Konovalov, and S. Ness. "Laser shaping of photonic materials: deep-ultraviolet and ultrafast lasers". In: Applied Surface Science 154-155 (2000), pp. 577-586.

[64] S. Pissadakis, L. Reekie, M. Hempstead, M.N. Zervas, and J.S. Wilkinson. "Relief gratings on Er/Yb-doped borosilicate glasses and waveguides by excimer laser ablation". In: Applied Surface Science 153.4 (2000), pp. 200-210.

[65] Ampere A. Tseng, Ying-Tung Chen, and Kung-Jeng Ma. "Fabrication of high-aspect-ratio microstructures using excimer laser". In: Optics and Lasers in Engineering 41.6 (2004), pp. 827-847.

[66] J. Bekesi, J. Meinertz, P. Simon, and J. Ihlemann. "Sub-500-nm patterning of glass by nanosecond KrF excimer laser ablation". In: Applied Physics A 110 (2013), pp. 17-21. 
[67] J. Meinertz, T. Fricke-Begemann, and J. Ihlemann. "Micron and Sub-Micron Gratings on Glass by UV Laser Ablation". In: Physics Procedia 41 (2013). Lasers in Manufacturing (LiM 2013), pp. 708-712.

[68] Igor A. Konovalov and Peter R. Herman. "Ablation-Induced Stresses in Fused

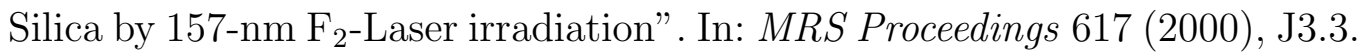

[69] Y. M. Xiao and Michael Bass. "Thermal stress limitations to laser fire polishing of glasses". In: Appl. Opt. 22.18 (Sept. 1983), pp. 2933-2936.

[70] Franck Guignard, Michel L. Autric, and Vincent Baudinaud. "Temperature and residual stress evolution in CO2-laser-irradiated glass". In: 3343 (1998).

[71] N. Borrelli, J. Helfinstine, J. Price, J. Schroeder, A. Streltsov, and J. Westbrook. "Glass strengthening with an ultrafast laser". In: International Congress on Applications of Lasers $\&$ Electro-Optics 2008.1 (2008), p. M404.

[72] Thomas Fricke-Begemann and Jürgen Ihlemann. "Direct light-coupling to thin-film waveguides using a grating-structured GRIN lens". In: Opt. Express 18.19 (Sept. 2010), pp. 19860-19866.

[73] Marcos Soldera, Sabri Alamri, Paul Alexander Sürmann, Tim Kunze, and Andrés Fabián Lasagni. "Microfabrication and Surface Functionalization of Soda Lime Glass through Direct Laser Interference Patterning". In: Nanomaterials 11.1 (2021).

[74] S. Isomae, M. Nanba, Y. Tamaki, and M. Maki. "Creep curve of silicon wafers". In: Applied Physics Letters 30.11 (1977), pp. 564-566.

[75] J. h. Jeong, D. Kwon, W.-S. Lee, and Y.-J. Baik. "Intrinsic stress in chemical vapor deposited diamond films: An analytical model for the plastic deformation of the Si substrate". In: Journal of Applied Physics 90 (2001), pp. 12271236.

[76] C. Saringer, M. Tkadletz, and C. Mitterer. "Restrictions of stress measurements using the curvature method by thermally induced plastic deformation of silicon substrates". In: Surface $\&$ Coatings Technology 274 (2015), pp. 6875 .

[77] S. R. J. Saunders, H. E. Evans, M. Li, D. D. Gohil, and S. Osgerby. "Oxidation Growth Stresses in an Alumina-Forming Ferritic Steel Measured by Creep Deflection". In: Oxidation of Metals 48.3./4. (1997), pp. 189-200.

[78] Cynthia G. Madras, Peter Y. Wong, Ioannis N. Miaoulis, Lee Goldman, and Ralph Korenstein. "Relaxation of extrinsic and intrinsic stresses in germanium substrates with silicon films". In: Thin Solid Films 320.2 (1998), pp. 260 -263 .

[79] S. E. Rosenberg, P. Y. Wong, and I. N. Miaoulis. "A theoretical study of the effect of thermal annealing on curvature changes in multilayered structures". In: Thin Solid Films 269 (1995), pp. 64-68.

[80] Q. Q. Chen, F. Z. Xuan, and S. T. Tu. "Residual stress analysis in the film/substrate system with the effect of creep deformation". In: Journal of Applied Physics 106.033512 (2009). 
[81] X. Dong, X. Feng, and K. C. Hwang. "Oxidation stress evolution and relaxation of oxide film/metal substrate system". In: Journal of Applied Physics 112.023502 (2012).

[82] Rudeger H. T. Wilke, Raegan L. Johnson-Wilke, Vincenzo Cotroneo, William N. Davis, Paul B. Reid, Daniel A. Schwartz, and Susan Trolier-McKinstry. "Sputter deposition of PZT piezoelectric films on thin glass substrates for adjustable x-ray optics". In: Appl. Opt. 52.14 (May 2013), pp. 3412-3419.

[83] Jürgen Ihlemann. "Patterning of oxide thin films by UV-laser ablation". In: Journal of Optoelectronics and Advanced Materials 7 (3 2005), pp. 11911195 .

[84] J. Ihlemann, J. Meinertz, and G. Danev. "Excimer laser ablation of thick SiOx-films: Etch rate measurements and simulation of the ablation threshold". In: Applied Physics Letters 101.9 (2012), p. 091901.

[85] Lukas Janos Richter, Clemens M. Beckmann, Jörg Meinertz, and Jürgen Ihlemann. "Laser Processing of Silicon Suboxide for the Fabrication of Multilevel Fused Silica Diffractive Phase Elements". In: Journal of Laser Micro/Nanoengineering 13 (3 2018), pp. 249-253.

[86] M. Jahn, J. Richter, R. Weichenhain-Schriever, J. Meinertz, and J. Ihlemann. "Ablation of silicon suboxide thin layers". In: Applied Physics A 101 (2010), pp. $533-538$.

[87] T. Fricke-Begemann, J. Meinertz, R. Weichenhain-Schriever, and J. Ihlemann. "Silicon suboxide $\left(\mathrm{SiO}_{x}\right)$ : laser processing and applications". In: $A p$ plied Physics A 117 (2014), pp. 13-18.

[88] Jürgen Ihlemann. "Micro patterning of fused silica by laser ablation mediated by solid coating absorption". In: Applied Physics A 93.1 (Oct. 2008), pp. 6568.

[89] J. Meinertz, L. J. Richter, C. M. Beckmann, and J. Ihlemann. "Quarzphasenmasken für Mikroskopie und Lasermaterialbearbeitung". In: Photonik 1 (2020).

[90] J. Ihlemann. Verfahren zur Herstellung optischer oder elektronischer Funktionselemente mit gekrümmter Oberfläche. German Patent, DE 102009016 113 B4. 2015.

[91] F.Z. Fang, X.D. Zhang, A. Weckenmann, G.X. Zhang, and C. Evans. "Manufacturing and measurement of freeform optics". In: CIRP Annals 62.2 (2013), pp. 823-846.

[92] Anita Winter, Elias Breunig, Peter Friedrich, and Laura Proserpio. "Progress on indirect glass slumping for future x-ray telescope optics". In: Space Telescopes and Instrumentation 2014: Ultraviolet to Gamma Ray. Ed. by Tadayuki Takahashi, Jan-Willem A. den Herder, and Mark Bautz. Vol. 9144. International Society for Optics and Photonics. SPIE, 2014, pp. 395 -402.

[93] Zhenbo Wei, Bing Ge, Xin Jin, Na Liu, Yingyu Liao, Bin Ma, Yuhong Bai, and Zhanshan Wang. "Development of manufacture of mirror glass substrate for x-ray timing and polarization observatory". In: Space Telescopes and Instrumentation 2016: Ultraviolet to Gamma Ray. Ed. by Jan-Willem A. den Herder, Tadayuki Takahashi, and Marshall Bautz. Vol. 9905. International Society for Optics and Photonics. SPIE, 2016, pp. 2100 -2111. 
[94] Heng E. Zuo, Youwei Yao, Brandon D. Chalifoux, Michael D. DeTienne, Ralf K. Heilmann, and Mark L. Schattenburg. "Recent progress on air-bearing slumping of segmented thin-shell mirrors for x-ray telescopes: experiments and numerical analysis". In: Optics for EUV, X-Ray, and Gamma-Ray Astronomy VIII. Ed. by Stephen L. O'Dell and Giovanni Pareschi. Vol. 10399. International Society for Optics and Photonics. SPIE, 2017, pp. 181-198.

[95] Helmut A. Schaeffer and Roland Langfeld. Werkstoff Glas - Alter Werkstoff mit großer Zukunft. Springer Vieweg, 2014.

[96] A. Wikström and P. Gudmundson. "Stresses in Thin Coatings from Curvature Measurements on Non-Planar Substrates". In: MRS Proceedings 653 (2000), Z10.4.1.

[97] Heraeus Quarzglas GmbH \& Co. KG. Quartz Glass for Optics - Data and Properties. Dec. 2019.

[98] R. Brückner. "Properties and structure of vitreous silica. I". In: Journal of Non-Crystalline Solids 5.2 (1970), pp. 123-175.

[99] Ted Pella Inc. Technical Information about GE 124 Quartz Material. https: //www.tedpella.com/histo_html/GE124-Quartz.htm. Accessed april 19, 2021.

[100] N. P. Bansal and R. H. Doremus. Handbook of Glass Properties. Academic Press Inc., 1986.

[101] Hans-Jürgen Kahlert. "UV Optics Systems for Excimer Lasers". In: Excimer Laser Technology: laser sources, optics, systems and applications. Ed. by Dirk Basting. Lambda Physik AG, 2001.

[102] Peter R. Herman and Klaus Mann. "Laser Beam Characterization". In: Excimer Laser Technology: laser sources, optics, systems and applications. Ed. by Dirk Basting. Lambda Physik AG, 2001.

[103] Reinhard Völkel und Maik Zimmermann. "Homogenisierung von Laserstrahlen". In: Photonik 3 (2006), pp. 76-79.

[104] Richard Leach. Fundamental Principles of Engineering Nanometrology. 2nd edition. Elsevier, 2014.

[105] Richard Leach and Han Haitjema. "Bandwidth characteristics and comparisons of surface texture measuring instruments". In: Measurement Science and Technology 21.3 (Jan. 2010), p. 032001.

[106] M. E. Thomas, M. P. Hartnett, and J. E. McKay. "The use of surface profilometers for the measurement of wafer curvature". In: Journal of Vacuum Science and Technology A 6.4 (1988), pp. 2570-2571.

[107] Documentation of the function Gaussian_filter1d of the module Ndimage of SciPy 0.19.1. https://docs.scipy.org/doc/scipy-0.19.1/reference/ generated/scipy.ndimage .gaussian_filter1d.html\#scipy.ndimage . gaussian_filter1d. Accessed September 18, 2020.

[108] I. N. Bronstein, K. A. Semendjajew, G. Musiol, and H. Mühlig. Taschenbuch der Mathematik. 7th edition. Verlag Harri Deutsch, 2008.

[109] J. N. Reddy. Theory and Analysis of Elastic Plates and Shells. 2nd edition. CRC Press, 2007. 
[110] J. N. Reddy. An Introduction to the Finite Element Method. 3th edition. McGraw-Hill International, 2005.

[111] L. Ridgway Scott. Introduction to Automated Modeling with FEniCS. Computational Modeling Initiative, LLC, 2018.

[112] Neil Gershenfeld. The Nature of Mathematical Modeling. Cambridge University Press, 1999.

[113] Anders Logg, Kent-Andre Mardal, Garth N. Wells, et al. Automated Solution of Differential Equations by the Finite Element Method. Springer, 2012.

[114] Martin S. Alnæs, Jan Blechta, Johan Hake, August Johansson, Benjamin Kehlet, Anders Logg, Chris Richardson, Johannes Ring, Marie E. Rognes, and Garth N. Wells. "The FEniCS Project Version 1.5". In: Archive of Numerical Software 3.100 (2015).

[115] Christophe Geuzaine and Jean-François Remacle. "Gmsh: A 3-D finite element mesh generator with built-in pre- and post-processing facilities". In: International Journal for Numerical Methods in Engineering 79.11 (2009), pp. 1309-1331.

[116] Anders Logg and Garth N. Wells. "DOLFIN: Automated Finite Element Computing". In: ACM Transactions on Mathematical Software 37.2 (2010).

[117] Hans Petter Langtangen and Anders Logg. Solving PDEs in Python - The FEniCS Tutorial Volume I. Springer, 2017.

[118] G. W. Scherer. Relaxation In Glass And Composites. John Wiley \& Sons, 1986.

[119] V. Janovský, S. Shaw, M.K. Warby, and J.R. Whiteman. "Numerical methods for treating problems of viscoelastic isotropic solid deformation". In: Journal of Computational and Applied Mathematics 63.1 (1995). Proceedings of the International Symposium on Mathematical Modelling and Computational Methods Modelling 94, pp. 91-107.

[120] Simon Shaw and J. R. Whiteman. "Computational Modelling of problems with memory with emphasis on viscoelastic solid deformation". In: unknown (1998). Found at https://www.researchgate.net/profile/Simon-Shaw2/publication/2647937_Computational_Modelling_of_problems_with_ memory_with_emphasis_on_viscoelastic_solid_deformation/links/ 02bfe5103e780ccc92000000 / Computational - Modelling - of - problems with-memory-with-emphasis-on-viscoelastic-solid-deformation . pdf Last access: 26th May 2021.

[121] Hans-Görg Roos and Hubert Schwetlick. Numerische Mathematik - Das Grundwissen für jedermann. B. G. Teubner, 1999.

[122] Nina Kristine Kylstad. "Simulating the viscoelastic response of the spinal cord". Master's thesis. Faculty of Mathematics and Natural Sciences, University of Oslo, 2014.

[123] Clemens M. Beckmann and Jürgen Ihlemann. "Figure correction of borosilicate glass substrates by nanosecond UV excimer laser irradiation". In: Opt. Express 28.13 (June 2020), pp. 18681-18692. 
[124] G. Thurner and R. Abermann. "Internal stress and structure of ultrahigh vacuum evaporated chromium and iron films and their dependence on substrate temperature and oxygen partial pressure during deposition". In: Thin Solid Films 192.2 (1990), pp. 277-285.

[125] R. Koch. "Stress in Evaporated and Sputtered Thin Films - A Comparison". In: Surface and Coatings Technology 204.12 (2010). Proceedings of the European Materials Research Socierty (E-MRS)Spring Meeting 2009, pp. 1973 -1982 .

[126] Youwei Yao, Brandon D. Chalifoux, Ralf K. Heilmann, Kai-Wing Chan, Hideyuki Mori, William W. Zhang, and Mark L. Schattenburg. "Thermal oxide patterning method for compensating coating stress in silicon x-ray telescope mirrors". In: Proceedings of SPIE 10699 (2018), p. 10699.

[127] Andreas Gödecke. "Grundlagenuntersuchungen zur Ablation von Gläsern mit ArF-Excimer-Laserstrahlung". Praxisprojektarbeit. Hochschule für angewandte Wissenschaft und Kunst Hildesheim/Holzminden/Göttingen - Fakultät Naturwissenschaften und Technik, 2013.

[128] Schott D263M Glass Coverslips Technical data. https://www . tedpella. com/histo_html/coverslip-info.htm. Accessed december 18, 2020.

[129] E. Matthias, M. Reichling, J. Siegel, O. W. Käding, S. Petzold, Skurk H., Bizenberger P., and E. Neske. "The Influence of Thermal Diffusion on Laser Ablation of Metal Films". In: Applied Physics A 58 (1994), pp. 129-136.

[130] S.R. Jackson, W.J. Metheringham, and P.E. Dyer. "Excimer laser ablation of Nd:YAG and Nd:glass". In: Applied Surface Science 86.1 (1995), pp. 223227.

[131] Jürgen Ihlemann, Malte Schulz-Ruhtenberg, and Thomas Fricke-Begemann. "Micro patterning of fused silica by ArF- and $\mathrm{F}_{2}$-laser ablation". In: Journal of Physics: Conference Series 59 (Apr. 2007), pp. 206-209.

[132] Jürgen Ihlemann. "Laser Micromachining". In: Laser Processing of Materials. Ed. by Peter Schaaf. Springer, 2010.

[133] D. Bäuerle. Laser Processing and Chemistry. 2nd ed. Springer, 1996.

[134] James E. Shelby. Introduction to Glass Science and Technology. 2nd edition. The Royal Society of Chemistry, 2005.

[135] W. N. Sharpe, J. Pulskamp, J. Gianola, C. Eberl, R. G. Polcawich, and R. J. Thompson. "Strain Measurement of Silicon Dioxide Microspecimens by Digital Imaging Processing". In: Experimental Mechanics 47 (2007), pp. 649658.

[136] Jinkui Chu and Duanqin Zhang. "Mechanical characterization of thermal $\mathrm{SiO}_{2}$ micro-beams through tensile testing". In: Journal of Micromechanics and Microengineering 19.9 (Sept. 2009), p. 095020.

[137] V. Hatty, H. Kahn, and A. H. Heuer. "Fracture Toughness, Fracture Strength, and Stress Corrosion Cracking of Silicon Dioxide Thin Films". In: Journal of Microelectromechanical Systems 17.4 (2008), pp. 943-947. 
[138] J.-P. Guin and Y. Gueguen. "Mechanical Properties of Glass". In: Springer Handbook of Glass. Ed. by J. D. Musgraves, J. Hu, and L. Calvez. Springer Nature Switzerland AG, 2019.

[139] S. M. Wiederhorn. "Influence of Water Vapor on Crack Propagation in SodaLime Glass". In: Journal of the American Ceramic Society 50.8 (1967), pp. 407414.

[140] S. M. Wiederhorn and L. H. Bolz. "Stress Corrosion and Static Fatigue of Glass". In: Journal of the American Ceramic Society 53.10 (1970), pp. 543548.

[141] Toshiyuki Tsuchiya, Atsuko Inoue, and Jiro Sakata. "Tensile testing of insulating thin films; humidity effect on tensile strength of $\mathrm{SiO}_{2}$ films". In: Sensors and Actuators A: Physical 82.1 (2000), pp. 286-290.

[142] Douglas I. Kushner and Michael A. Hickner. "Water Sorption in ElectronBeam Evaporated $\mathrm{SiO}_{2}$ on QCM Crystals and Its Influence on Polymer Thin Film Hydration Measurements". In: Langmuir 33.21 (2017). PMID: 28474891, pp. 5261-5268.

[143] Akira Shintani, Shojiro Sugaki, and Hisao Nakashima. "Temperature dependence of stresses in chemical vapor deposited vitreous films". In: Journal of Applied Physics 51.8 (1980), pp. 4197-4205.

[144] M. Kakehata, H. Yashiro, A. Oyane, and A. Ito. "Anisotropic surface stress on yttria-stabilized zirconia ceramics with femtosecond-laser induced periodic surface structures". 21st International Symposium on Laser Precision Microfabrication. 2020.

[145] Y. Huang, D. Ngo, X. Feng, and A. J. Rosakis. "Anisotropic, non-uniform misfit strain in a thin film bonded on a plate substrate". In: Interaction and Multiscale Mechanics 1.1 (2007), pp. 123 -142.

[146] A. E. Hill and G. R. Hoffman. "Stress in films of silicon monoxide". In: British Journal of Applied Physics 18 (1967), pp. 13-22.

[147] Chun-Hway Hsueh and Sanboh Lee. "Effects of viscous flow on residual stresses in film/substrate systems". In: Journal of Applied Physics 91.5 (2002), pp. 2760-2765.

[148] A. Fargeix and G. Ghibaudo. "Dry oxidation of silicon: A new model of growth including relaxation of stress by viscous flow". In: Journal of Applied Physics 54.12 (1983), pp. 7153-7158.

[149] A. Fargeix and G. Ghibaudo. "Densification of thermal $\mathrm{SiO}_{2}$ due to intrinsic oxidation stressing". In: Journal of Physics D: Applied Physics 17 (1984), pp. 2331-2336.

[150] J. T. Fitch, C. H. Bjorkman, G. Lucovsky, F. H. Pollak, and X. Yin. "Intrinsic stress and stress gradients at the $\mathrm{SiO}_{2} / \mathrm{Si}$ interface in structures prepared by thermal oxidation of $\mathrm{Si}$ and subjected to rapid thermal annealing". In: Journal of Vacuum Science 85 Technology B: Microelectronics Processing and Phenomena 7.4 (1989), pp. 775-781. 
[151] O. B. Loopstra, E. R. van Snek, Th. H. de Keijser, and E. J. Mittemeijer. "Model for stress and volume changes of a thin film on a substrate upon annealing: Application to amorphous Mo/Si multilayers". In: Phys. Rev. B 44 (24 Dec. 1991), pp. 13519-13533.

[152] A. Witvrouw, C.A. Volkert, and F. Spaepen. "The viscosity of amorphous metallic thin films". In: Materials Science and Engineering: A 134 (1991). Proceedings of the Seventh International Conference on Rapidly Quenched Materials, pp. 1274-1277.

[153] Georg Hass. "Preparation, Structure, and Applications of Thin Films of Silicon Monoxide and Titanium Dioxide". In: Journal of the American Ceramic Society 33.12 (1950), pp. 353-360.

[154] Georg Hass and Calvin D. Salzberg. "Optical Properties of Silicon Monoxide in the Wavelength Region from 0.24 to 14.0 Mircrons". In: Journal of the Optical Society of America 44.3 (1954), pp. 181-187.

[155] E. Cremer and H. Pulker. "Herstellung und Analyse von SiO-Aufdampfschichten verschiedener optischer Eigenschaften". In: Monatshefte für Chemie 93.2 (1962), pp. 491-496.

[156] M. Nakamura, Y. Mochizuki, K. Usami, Y. Itoh, and T. Nozaki. "Infrared absorption spectra and compositions of evaporated silicon oxides $\left(\mathrm{SiO}_{x}\right)$ ". In: Solid State Communications 50.12 (1984), pp. 1079 -1081.

[157] E. Fogarassy, A. Slaoui, C. Fuchs, and J. L. Regolini. "Rapid thermal oxidation of silicon monoxide". In: Applied Physics Letters 51.5 (1987), pp. 337339.

[158] N. V. Sopinskii and A. V. Russu. "Ellipsometric study on the formation of nanocomposites by annealing $\mathrm{SiO}_{x}$ films in oxygen-containing media". In: $O p$ toelectronics, Instrumentation and Data Processing 51.4 (July 2015), pp. 423427.

[159] R. Le Parc, C. Levelut, J. Pelous, V. Martinez, and B. Champagnon. "Influence of fictive temperature and composition of silica glass on anomalous elastic behaviour". In: Journal of Physics: Condensed Matter 18.32 (July 2006), pp. 7507-7527.

[160] Mary F. Doerner and William D. Nix. "Stresses and deformation processes in thin films on substrates". In: Critical Reviews in Solid State and Materials Sciences 14.3 (1988), pp. 225-268.

[161] A. La Magna, G. Nicotra, C. Bongiorno, C. Spinella, M. G. Grimaldi, E. Rimini, L. Caristia, and S. Coffa. "Role of the internal strain on the incomplete $\mathrm{Si} / \mathrm{SiO}_{2}$ phase separation in substoichiometric silicon oxide films". In: Applied Physics Letters 90.18 (2007), p. 183101.

[162] G. Zatryb, J. Misiewicz, P.R.J. Wilson, J. Wojcik, P. Mascher, and A. Podhorodecki. "Stress transition from compressive to tensile for silicon nanocrystals embedded in amorphous silica matrix". In: Thin Solid Films 571 (2014), pp. 18-22.

[163] Warren J. Smith. Practical Optical System Layout - And Use of Stock Lenses. McGraw-Hill, 1997. 
[164] J. Pivot. "Mechanical properties of $\mathrm{SiO}_{x}$ thin films". In: Thin Solid Films 89.2 (1982), pp. 175-190.

[165] Chien-Jih Han and C. Robert Helms. "Parallel Oxidation Mechanism for Si Oxidation in Dry $\mathrm{O}_{2}$ ". In: Journal of The Electrochemical Society 134.5 (1987), pp. 1297-1302.

[166] A. Reisman, E. H. Nicollian, C. K. Williams, and C. J. Merz. "The modelling of silicon oxidation from $1 \times 10^{-5}$ to 20 atmospheres". In: Journal of Electronic Materials 16.1 (Jan. 1987), pp. 45-55.

[167] D. Bauza. "thermal oxidation of silicon and $\mathrm{Si}_{-} \mathrm{SiO}_{2}$ interface morphology, structure and localized states". In: Handbook of surfaces and interfaces of materials Vol.1 - Surface and interface phenomena. Ed. by H. S. Nalwa. Academic Press, 2001.

[168] L. C. Feldman. "Introduction". In: Fundamental Aspects of Silicon Oxidation. Ed. by Y. J. Chabal. Springer, 2001.

[169] F. Jansen, M. A. Machonkin, N. Palmieri, and D. Kuhman. "Thermomechanical properties of amorphous silicon and nonstoichiometric silicon oxide films". In: Journal of Applied Physics 62.12 (1987), pp. 4732-4736.

[170] O. M. Feroughi, C. Sternemann, Ch. J. Sahle, M. A. Schroer, H. Sternemann, H. Conrad, A. Hohl, G. T. Seidler, J. Bradley, T. T. Fister, M. Balasubramanian, A. Sakko, K. Pirkkalainen, K. Hämäläinen, and M. Tolan. "Phase separation and Si nanocrystal formation in bulk SiO studied by x-ray scattering". In: Applied Physics Letters 96.8 (2010), p. 081912.

[171] S. Hernandez, P. Miska, M. Grün, S. Estrade, F. Peiro, B. Garrido, M. Vergnat, and P. Pellegrino. "Tailoring the surface density of silicon nanocrystals embedded in $\mathrm{SiO}_{x}$ single layers". In: Journal of Applied Physics 114.23 (2013), p. 233101.

[172] R Roscoe. "The viscosity of suspensions of rigid spheres". In: British Journal of Applied Physics 3.8 (Aug. 1952), pp. 267-269.

[173] Shaocheng Ji. "A generalized mixture rule for estimating the viscosity of solid-liquid suspensions and mechanical properties of polyphase rocks and composite materials". In: Journal of Geophysical Research: Solid Earth 109.B10 (2004).

[174] Lyubov V. Titova, Tyler L. Cocker, David G. Cooke, Xiongyao Wang, Al Meldrum, and Frank A. Hegmann. "Ultrafast percolative transport dynamics in silicon nanocrystal films". In: Phys. Rev. B 83 (8 Feb. 2011), p. 085403.

[175] A. N. Goldstein. "The melting of silicon nanocrystals: Submicron thin-film structures derived from nanocrystal precursors". In: Applied Physics A 62 (1996), pp. 33-37.

[176] Leonid Khriachtchev, Markku Räsänen, and Sergei Novikov. "Laser-controlled stress of Si nanocrystals in a free-standing $\mathrm{Si} / \mathrm{SiO}_{2}$ superlattice". In: Applied Physics Letters 88.1 (2006), p. 013102.

[177] J. Priest and H. L. Caswell. "Annealing of residual stress in silicon monoxide films". In: British Journal of Applied Physics 12 (1961), pp. 580-581. 
[178] H. Coffin, C. Bonafos, S. Schamm, N. Cherkashin, G. Ben Assayag, A. Claverie, M. Respaud, P. Dimitrakis, and P. Normand. "Oxidation of Si nanocrystals fabricated by ultralow-energy ion implantation in thin $\mathrm{SiO}_{2}$ layers". In: Journal of Applied Physics 99.4 (2006), p. 044302.

[179] L. M. Landsberger and W. A. Tiller. "Refractive index, relaxation times and the viscoelastic model in dry-grown $\mathrm{SiO}_{2}$ films on Si". In: Applied Physics Letters 51.18 (1987), pp. 1416-1418.

[180] E. Kobeda and E. A. Irene. " $\mathrm{SiO}_{2}$ film stress distribution during thermal oxidation of Si". In: Journal of Vacuum Science and Technology B 6.2 (1988), pp. $574-578$.

[181] S. M. Rekhson. In: Soviet Journal of Glass Physics and Chemistry 1.5 (1975), pp. $417-421$.

[182] R. Brüning, C. Levelut, R. Le Parc, A. Faivre, L. Semple, M. Vallee, J.P. Simon, and J.-L. Hazemann. "Temperature scanning small angle x-ray scattering measurements of structural relaxation in type-III vitreous silica". In: Journal of Applied Physics 102.8 (2007), p. 083535.

[183] Feng Huang, Qiuming Song, Ming Li, Bin Xie, Haiqian Wang, Yousong Jiang, and Yizhou Song. "Influences of annealing temperature on the optical properties of $\mathrm{SiO}_{x}$ thin film prepared by reactive magnetron sputtering". In: Applied Surface Science 255.5, Part 1 (2008), pp. 2006 -2011.

[184] Nicolae Tomozeiu. "Effects of UV photon irradiation on $\mathrm{SiO}_{x}(0<x<2)$ structural properties". In: Applied Surface Science 253.1 (2006). Proceedings of the E-MRS 2005 Spring Meeting Symposium P: Current trends in optical and X-ray metrology of advanced materials for nanoscale devices, pp. 376380 .

[185] T.-S. Park and S. Suresh. "Effects of line and passivation geometry on curvature evolution during processing and thermal cycling in copper interconnect lines". In: Acta Materialia 48.12 (2000), pp. 3169-3175.

[186] Nan Wang, Thomas Fricke-Begemann, Patrick Peretzki, Karsten Thiel, Jürgen Ihlemann, and Michael Seibt. "Microstructural analysis of the modifications in substrate-bound silicon-rich silicon oxide induced by continuous wave laser irradiation". In: Journal of Alloys and Compounds 707 (2017). Selected papers presented at ISMANAM 2016, July 3rd-8th, Nara, Japan, pp. 227-232.

[187] A. Agarwal and M. Tomozawa. "Surface and bulk structural relaxation kinetics of silica glass". In: Journal of Non-Crystalline Solids 209 (1997), pp. 264272.

[188] M. Tomozawa, D. Kim, A. Agarwal, and K. M. Davis. "Water diffusion and surface structural relaxation of silica glasses". In: Journal of Non-Crystalline Solids 288 (2001), pp. 73-80.

[189] A. Koike and M. Tomozawa. "Size effect on surface structural relaxation kinetics of silica glass sample". In: Journal of Non-Crystalline Solids 352.36 (2006), pp. $3787-3793$. 
[190] Craig R. Forest, Claude R. Canizares, Daniel R. Neal, Michael McGuirk, and Mark Lee Schattenburg. "Metrology of thin transparent optics using ShackHartmann wavefront sensing". In: Optical Engineering 43 (2004), pp. 742753.

[191] E. P. EerNisse. "Viscous flow of thermal $\mathrm{SiO}_{2}$ ". In: Applied Physics Letters 30.6 (1977), pp. 290-293.

[192] V. M. Burlakov, G. A. D. Briggs, A. P. Sutton, Angelo Bongiorno, and Alfredo Pasquarello. "Modeling Phase Separation in Nonstoichiometric Silica". In: Phys. Rev. Lett. 93 (13 Sept. 2004), p. 135501.

[193] Joel P. McDonald, Vanita R. Mistry, Katherine E. Ray, Steven M. Yalisove, John A. Nees, and Neville R. Moody. "Femtosecond-laser-induced delamination and blister formation in thermal oxide films on silicon (100)". In: Applied Physics Letters 88.15 (2006), p. 153121.

[194] Philippe Goudeau, Pascale Villain, Nobumichi Tamura, and Howard A. Padmore. "Mesoscale x-ray diffraction measurement of stress relaxation associated with buckling in compressed thin films". In: Applied Physics Letters 83.1 (2003), pp. 51-53.

[195] Haikun Jia, Shi Bin Wang, Nobumichi Tamura, and Philippe Goudeau. "Stress Relaxation Related to Spontaneous Thin Film Buckling: Correlation between Finite Element Calculations and Micro Diffraction Analysis". In: Quantum Beam Science 3.1 (Dec. 2018), p. 1.

[196] Schott AG. Ultra-Thin Glass for Electronics Applications. https : //www . schott . com/d/corporate/ac4005ef-6c05-45a1-bce8-b52379aecf26/ 1.0 /schott-ultra-thin-glass-electronics - appl-datasheet-en2019.pdf. Accessed february 11, 2021.

[197] Schott AG. Specification - Physical and chemical properties (PCP) D263 bio. https : / / www . schott . com/d / corporate / e13eac2e-f36f-4ead-bc0fce3038bcd866/1.1/schott_d_263_bio_pcp_en.pdf. Accessed february 11, 2021. 



\section{Acknowledgments}

Many people contributed to this work, and I feel that they should be named and deserve thanks. First of all, I would like to thank my supervisor and group leader Jürgen Ihlemann for his advice, patience, many fruitful discussions and at last also proofreading of the manuscript. I am always impressed by his optimism and calmness in all kind of questions and by his ability of promoting an always positive, constructive and quite communicative atmosphere inside the group. I also achieved much support from my colleagues Jörg Meinertz and Lukas Janos Richter. They helped me with advice, discussions and support in the laboratory. Janos regularly deposited thin films for me, and a few times I could take advantage of optical setups Jörg or Janos had installed. I would also like to thank my master students Max Buczek and Laura Fütterer for their contributions to our scientific questions. Jens Oltmanns contributed with discussions and AFM measurements.

I am grateful to my thesis advisory committee, consisting of Alexander Egner, Michael Seibt and Ulrich Vetter, for their support. Special thanks goes to Uli for taking the time of more frequent meetings. Prof. Egner deserves special thanks for being also a member of my examination committee. In this regard I would also especially like to thank Prof. Hofsäss, who kindly agreed to be the second member of my examination committee.

Outside physics, I really need to thank my parents for all their support, directly and indirectly, during my whole life until now. And I also want to thank my brother and the rest of my family. At last, I would like to thank Sabine Grosse for caring, distracting, bearing and everything else. 